\title{
Modelo bayesiano para dados de sobrevivência com riscos semicompetitivos baseado em cópulas
}

\section{Elizabeth González Patiño}

\author{
TESE APRESENTADA \\ $\mathrm{AO}$ \\ Instituto DE MATEMÁtica e Estatística \\ DA \\ Universidade DE SÃo PAUlo \\ PARA \\ OBTENÇÃO DO TÍTULO \\ $\mathrm{DE}$ \\ DOUTOR EM CIÊNCIAS \\ Programa: Estatística \\ Orientadora: Prof. Dr. Gisela Tunes
}

Durante o desenvolvimento deste trabalho o autor recebeu auxílio financeiro da CAPES

-São Paulo, março de 2018- 


\title{
Modelo bayesiano para dados de sobrevivência com riscos semicompetitivos baseado em cópulas
}

\author{
Esta versão da dissertação/tese contém as correções e alterações sugeridas \\ pela Comissão Julgadora durante a defesa da versão original do trabalho, \\ realizada em 23/03/2018. Uma cópia da versão original está disponível no \\ Instituto de Matemática e Estatística da Universidade de São Paulo.
}

Comissão Julgadora:

- Prof ${ }^{a}$. Dr ${ }^{\mathrm{a}}$. Gisela Tunes da Silva (orientadora) - IME-USP

- Prof. Dr. Nelson Ithiro Tanaka - IME-USP

- Prof. Dr. Antonio Carlos Pedroso de Lima - IME-USP

- Prof. Dr. Jóse Santos Romeo Núñez - Universdad de Santiago de Chile

- Prof. Dr. Mário de Castro Andrade Filho - ICMC-USP 
A mis amores Fidelito y Cristián.

A mi linda família desde la distancia. 


\section{Agradecimentos}

Agradeço a meus orientadores a profa Gisela Tunes e o prof Nelson Tanaka pela confiança, paciência, apoio e dedicação.

Aos meus queridos Fidelito e Cris, eles são os meus motores que colocam cada dia um sorriso no meu coração. A minha familia na Colômbia que torceram todo este tempo para dar tudo certo.

A todos os meus colegas e/ou amigos do IME-USP (incluíndo colombianos, chilenos e brasileiros) pelos estudos, convivência, boas experiências e em geral, pelo carinho que fizeram minha vida no Brasil muito mais agradável e feliz.

A todos os professores do Instituto que de uma u outra forma contribuíram na minha formação acadêmica e pessoal.

Um profundo agradecimento a todos os professores membros da banca pelas sugestões, observações e correções. Valiosos incentivos para dar continuidade ao trabalho e seguir na pesquisa.

Agradeço também à agência de fomento Capes, pelo auxílio financieiro.

Finalmente, obrigada Brasil! 


\section{Resumo}

PATIÑO, E. G. Modelo bayesiano para dados de sobrevivência com riscos semicompetitivos baseado em cópulas. 2018. Tese (Doutorado) - Instituto de Matemática e Estatística, Universidade de São Paulo, São Paulo, 2018.

Motivados por um conjunto de dados de pacientes com insuficiência renal crônica (IRC), propomos uma nova modelagem bayesiana que envolve cópulas da família Arquimediana e um modelo misto para dados de sobrevivência com riscos semicompetitivos. A estrutura de riscos semicompetitivos é bastante comum em estudos clínicos em que dois eventos são de interesse, um intermediário e outro terminal, de forma tal que a ocorrência do evento terminal impede a ocorrência do intermediário mas não vice-versa. Nesta modelagem provamos que a distribuição a posteriori sob a cópula de Clayton é própria. Implementamos os algoritmos de dados aumentados e amostrador de Gibbs para a inferência bayesiana, assim como os criterios de comparação de modelos: LPML, DIC e BIC. Realizamos um estudo de simulação para avaliar o desempenho da modelagem e finalmente aplicamos a metodologia proposta para analisar os dados dos pacientes com IRC, além de outros de pacientes que receberam transplante de medula óssea.

Palavras-chave: Copulas Arquimedianas, Riscos semicompetitivos, Modelo misto, Inferência Bayesiana, Amostrador de Gibbs. 


\section{Abstract}

PATIÑO, E. G. Bayesian model for survival data with semicompeting risks based on copulas. 2018. Tese (Doutorado) - Instituto de Matemática e Estatística, Universidade de São Paulo, São Paulo, 2010.

Motivated by a dataset of patients with chronic kidney disease (CKD), we propose a new bayesian model including the Arquimedean copula and a mixed model for survival data with semicompeting risks. The structure of semicompeting risks appears frequently in clinical studies where two-types of events are involved: a nonterminal and a terminal event such that the occurrence of terminal event precludes the occurrence of the nonterminal event but not viceversa. In this work we prove that the posterior distribution is proper when the Clayton copula is used. We implement the data augmentation algorithm and Gibbs sampling for the bayesian inference, as well as some bayesian model selection criteria: LPML, BIC and DIC. We carry out a simulation study for assess the model performance and finally, our methodology is illustrated with the chronic kidney disease study.

Keywords: Arquimedean copula, Semicompeting risks, Mixed model, Bayesian inference, Gibbs sampling. 


\section{Sumário}

Lista de Abreviaturas $\quad$ xi

Lista de Símbolos $\quad$ xiii

Lista de Figuras $\quad$ Xv

Lista de Tabelas $\quad$ xix

1 Introdução 1

1.1 Revisão da literatura . . . . . . . . . . . . . . . . . . . . . . . 2

1.2 Motivação . . . . . . . . . . . . . . . . . . . . . . . 4

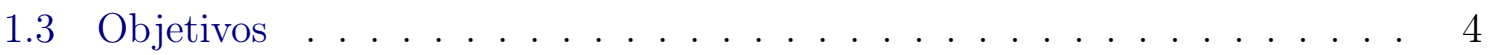

1.4 Contribuições . . . . . . . . . . . . . . . . . . . . 5

1.5 Organização do trabalho . . . . . . . . . . . . . . . . . 5

$\begin{array}{lll}2 & \text { Conceitos preliminares } & 7\end{array}$

2.1 Riscos semicompetitivos . . . . . . . . . . . . . . . . 7

2.2 Cópulas e estrutura de dependência . . . . . . . . . . . . . . . . 8

2.2 .1 Tau de Kendall . . . . . . . . . . . . . . . . . . . . . . . . . . 9 9

2.2.2 Cópula de Clayton . . . . . . . . . . . . . . . . . . . . . . 10

2.2.3 Cópula de Frank . . . . . . . . . . . . . . . . . . . . . . . . . 11

2.2.4 Cópula de Gumbel ou fragilidade estável positiva . . . . . . . . . 12

2.3 Análise descritiva para dados com riscos semicompetitivos . . . . . . . . 14

2.3.1 Estudo da função de sobrevivência por meio de cópulas . . . . . . 14

3 Modelo de regressão bayesiano para riscos semicompetitivos $\quad 19$

3.1 Modelagens propostas . . . . . . . . . . . . . . . 20

3.1.1 Função de verossimilhança . . . . . . . . . . . . . . . . . . . . 24 
3.1 .2 Inferência bayesiana . . . . . . . . . . . . . . . . . . 28

3.2 Cópula de Clayton . . . . . . . . . . . . . . . . . . . . . . 31

3.2.1 A distribuição a posteriori é própria? . . . . . . . . . . . . . . . . 40

3.3 Cópula de Frank . . . . . . . . . . . . . . . . . . . . . . . . . 42

3.4 Cópula de Gumbel . . . . . . . . . . . . . . . . . . . . . . 50

4 Aspectos computacionais e estudo de simulação 59

4.1 Amostragem . . . . . . . . . . . . . . . . . . . . . . . . . 59

4.2 Estudo de simulação . . . . . . . . . . . . . . . . . . . . . . . 61

4.2.1 Geração das amostras . . . . . . . . . . . . . . . . . . . 63

4.2.2 Resultados para a cópula de Clayton . . . . . . . . . . . . . . . 66

5 Aplicação $\quad 71$

5.1 Comparação de modelos . . . . . . . . . . . . . . . . . . . . . . . 71

5.2 Transplante de medula óssea $(\mathrm{TMO}) \ldots \ldots$. . . . . . . . . . . . 73

5.2.1 Ajuste da modelagem proposta . . . . . . . . . . . . . 74

5.3 Insuficiência renal crônica $($ IRC) ～. . . . . . . . . . . . . . . . 78

5.3.1 Ajuste da modelagem proposta . . . . . . . . . . . 81

6 Conclusões $\quad 91$

6.1 Considerações finais . . . . . . . . . . . . . . . . . . . . . . 9 91

6.2 Sugestões para pesquisas futuras . . . . . . . . . . . . . . . . . . 92

A Função de verossimilhança: Expressões para sua construção usando a cópula de Clayton $\quad 95$

$\begin{array}{ll}\text { B Prova do teorema } 1 & 101\end{array}$

C Tabelas e figuras das simulações $\quad 115$

D Figuras das aplicações 139

D.1 Transplante de medula óssea $(\mathrm{TMO})$. . . . . . . . . . . . . . . . . . . 140

D.2 Insuficiência renal crônica (IRC) . . . . . . . . . . . . . . . . . . . . . 144

$\begin{array}{ll}\text { Referências Bibliográficas } & 153\end{array}$ 


\section{Lista de Abreviaturas}

ACF Função de autocorrelação

(Autocorrelation function)

ARMS Algoritmo de rejeição adaptativa de amostragem Metropolis (Adaptative rejection Metropolis sampling)

ARS Algoritmo de rejeição adaptativa

(Adaptative rejection sampling)

ALPML Pseudoverossimilhança marginal relativa

(Relative pseudomarginal likelihood)

BIC Critério de informação bayesiano

(Bayesian information criterion)

CP Probabilidade de cobertura

(Coverage probability)

CPO Ordenadas preditivas condicionais

(Conditional predictive ordinates)

DIC Critério de informação da desviância

(Deviance information criterion)

EA Efeito aleatório

(Random effect)

EBMT Sociedade Europeia para sangue e transplante de medula

(European society for blood and marrow transplantation)

EMV Estimador de máxima verossimilhança

(Maximum likelihood estimator)

ESRD Ultimos estágios da doença renal

(End stage renal disease)

HPD Intervalo de maior mensidade a posteriori

(Highest posterior density interval)

ICC Insuficiência cardíaca congestiva

(Congestive heart failure) 
IRC Insuficiência renal crônica

(Cronical kidney disease)

LPML Logaritmo da pseudoverossimilhança marginal

(Logarithm of the pseudomarginal likelihood)

MCMC Monte Carlo via cadeias de Markov

(Markov chain Monte Carlo)

PVF Função pôtencia de variância

(Power variance function)

RMSE Raiz quadrada do erro quadrático médio

(Square root of mean square error)

SD Desvio padrão

(Standard deviation)

TMO Transplante de medula óssea

(Bone marrow transplant (BMT)) 


\section{Lista de Símbolos}

$\mathcal{C}_{\theta} \quad$ Cópula de parâmetro $\theta$.

$\tau_{\theta} \quad$ Coeficiente tau de Kendall como função de $\theta$.

$\Omega^{\boldsymbol{\xi}} \quad$ Espaço paramétrico de $\boldsymbol{\xi}$.

$\mathbb{R} \quad$ Conjunto dos números reais.

$\mathbb{E}(X) \quad$ Valor esperado da variável $X$.

$\operatorname{Pr}(\cdot) \quad$ Função de probabilidade.

$\mathbb{I}(A) \quad$ Função indicadora do conjunto $A$.

$I_{a} \quad$ Matriz identidade de ordem $a$.

i.i.d. Independiente e identicamente distribuído. 


\section{Lista de Figuras}

2.1 Função distribuição (esquerda) e função densidade (direita) da cópula de Clayton $\operatorname{com} \theta=1(\tau=0,333) \ldots \ldots \ldots \ldots \ldots$

2.2 Função distribuição (esquerda) e função densidade (direita) da cópula de Frank $\operatorname{com} \theta=4(\tau \simeq 0,388) \ldots \ldots \ldots \ldots \ldots$. . . . . . . . . . . . . . . . . .

2.3 Função distribuição (esquerda) e função densidade (direita) da cópula de Gumbel com $\theta=1,5(\tau=0,333) \ldots \ldots \ldots \ldots$

3.1 Diagrama com as variáveis chaves das modelagens propostas na estrutura de riscos semicompetitivos. . . . . . . . . . . . . . . . . . . 21

5.1 Diagrama de dispersão dos tempos até a ocorrência dos eventos de interesse, recaída e óbito, dos dados de TMO. Incluí sómente os indivíduos do caso 2 . 75

5.2 Função densidade a posteriori, traços e ACF para o parâmetro da cópula de Clayton do ajuste da modelagem fixa com $\left(J_{0}, J_{1}, J_{2}\right)=(15,15,15)$ aos dados de TMO . . . . . . . . . . . . . . . . . . 76

5.3 Diagrama de dispersão dos tempos até a ocorrência dos eventos de interesse, progressão e óbito, dos dados de IRC. Incluí sómente os indivíduos do caso 2. 81

5.4 Funções de sobrevivência pelo estimador cópula gráfico para o tempo (em dias) até a progressão (esquerda) e Kaplan-Meier para o tempo (em dias) até o óbito (direita) . . . . . . . . . . . . . . . . . 81

5.5 Funções densidade a posteriori, traço e ACF para o parâmetro da cópula $\theta$ do ajuste do modelo fixo com $\left(J_{0}, J_{1}, J_{2}\right)=(2,2,2)$ aos dados de IRC . . . . . 86

5.6 Função densidade a posteriori, traço e ACF para o parâmetro da cópula $\theta$ (esquerda) e a variância do efeito aleatório $B$ (direita) do ajuste do modelo misto com $\left(J_{0}, J_{1}, J_{2}\right)=(1,1,1)$ aos dados de IRC. 
C.1 Média das médias e desvios padrão a posteriori das 200 réplicas de tamanhos $N=500$ (linha contínua) e $N=1000$ (linha pontilhada) para os parâmetros $\beta_{0}$, associado ao tratamento $A$, e $\gamma_{01}$ associado a $X_{1}$ do modelo $T_{D}$ segundo a cópula de Clayton. A linha horizontal representa o valor verdadeiro do parâmetro.127

C.2 Média das médias e desvios padrão a posteriori das 200 réplicas de tamanhos $N=500$ (linha contínua) e $N=1000$ (linha pontilhada) para o parâmetro $\gamma_{02}$ associado a $X_{2}$ do modelo $T_{D}$ segundo a cópula de Clayton. A linha horizontal representa o valor verdadeiro do parâmetro. . . . . . . . . . . . . . . 128

C.3 Média das médias e desvios padrão a posteriori das 200 réplicas de tamanhos $N=500$ (linha contínua) e $N=1000$ (linha pontilhada) para os parâmetros $\beta_{1}$, associado ao tratamento $A$, e $\gamma_{11}$ associado a $X_{1}$ do modelo $T_{E}$ segundo a cópula de Clayton. A linha horizontal representa o valor verdadeiro do parâmetro.129

C.4 Média das médias e desvios padrão a posteriori das 200 réplicas de tamanhos $N=500$ (linha contínua) e $N=1000$ (linha pontilhada) para o parâmetro $\gamma_{12}$, associado a $X_{2}$ do modelo $T_{E}$ segundo a cópula de Clayton. A linha horizontal representa o valor verdadeiro do parâmetro. . . . . . . . . . . . . . . . . . . 130

C.5 Média das médias e desvios padrão a posteriori das 200 réplicas de tamanhos $N=500$ (linha contínua) e $N=1000$ (linha pontilhada) para os parâmetros $\beta_{21}$, associado ao tratamento $A$, e $\beta_{22}$ associado a mudança de trantamento $V(1-A)$ do modelo $T_{G}$ segundo a cópula de Clayton. A linha horizontal representa o valor verdadeiro do parâmetro. . . . . . . . . . . . . . . . . . . 131

C.6 Média das médias e desvios padrão a posteriori das 200 réplicas de tamanhos $N=500$ (linha contínua) e $N=1000$ (linha pontilhada) para os parâmetros $\gamma_{21}$ e $\gamma_{22}$ associados às variáveis $X_{1}$ e $X_{2}$, respectivamente, do modelo $T_{G}$ segundo a cópula de Clayton. A linha horizontal representa o valor verdadeiro do parâmetro. . . . . . . . . . . . . . . . . . . . . . . . . . . 132

C.7 Média das médias e desvios padrão a posteriori das 200 réplicas de tamanhos $N=500$ (linha contínua) e $N=1000$ (linha pontilhada) para o parâmetro $\gamma_{23}$ associado à variável $Z$ do modelo $T_{G}$ segundo a cópula de Clayton. A linha horizontal representa o valor verdadeiro do parâmetro. . . . . . . . . . . . . . 133

C.8 Média da médias e desvios padrão a posteriori das 200 réplicas de tamanhos $N=500$ (linha contínua) e $N=1000$ (linha pontilhada) para os parâmetros $\alpha_{0}$ e $\alpha_{1}$, associados ao intercepeto e ao tratamento $A$ do modelo logito segundo a cópula de Clayton. A linha horizontal representa o valor verdadeiro do parâmetro.134 
C.9 Média das médias e desvios padrão a posteriori das 200 réplicas de tamanhos $N=500$ (linha contínua) e $N=1000$ (linha pontilhada) para os parâmetros $\alpha_{2}$ e $\alpha_{3}$ associados ás variáveis $X_{1}$ e $X_{2}$ do modelo logito segundo a cópula de Clayton. A linha horizontal representa o valor verdadeiro do parâmetro. . . . . 135

C.10 Média das médias e desvios padrão a posteriori das 200 réplicas de tamanhos $N=500$ (linha contínua) e $N=1000$ (linha pontilhada) para o parâmetro da cópula $\theta$ segundo a cópula de Clayton. A linha horizontal representa o valor verdadeiro do parâmetro. . . . . . . . . . . . . . . . . . . . . 136

C.11 Média das médias e desvios padrão a posteriori das 200 réplicas de tamanhos $N=500$ (linha contínua) e $N=1000$ (linha pontilhada) para a variância do efeito aleatório $B$ segundo a cópula de Clayton. A linha horizontal representa o valor verdadeiro do parâmetro. . . . . . . . . . . . . . . . . . . . . 137

D.1 Densidades a posteriori, traços e ACFs para a componente $T_{D}$ (óbito) do ajuste do modelo fixo com $\left(J_{0}, J_{1}, J_{2}\right)=(15,15,15)$ segundo a cópula de Clayton aos dados de TMO. . . . . . . . . . . . . . . . . . . . . . . . . . . . . 140

D.2 Densidades a posteriori, traços e ACFs para a componente $T_{E}$ (recaída) do ajuste do modelo fixo com $\left(J_{0}, J_{1}, J_{2}\right)=(15,15,15)$ segundo a cópula de Clayton aos dados de TMO. . . . . . . . . . . . . . . . . . . . . . 141

D.3 Densidades a posteriori, traços e ACFs para a componente $T_{G}$ (óbito após recaída) do ajuste do modelo fixo com $\left(J_{0}, J_{1}, J_{2}\right)=(15,15,15)$ segundo a cópula de Clayton aos dados de TMO . . . . . . . . . . . . . . . . . . . . . . 142

D.4 Densidades a posteriori, traços e ACFs para a componente logito do ajuste do modelo fixo com $\left(J_{0}, J_{1}, J_{2}\right)=(15,15,15)$ segundo a cópula de Clayton aos dados de TMO . . . . . . . . . . . . . . . . . . . . . . . . 143

D.5 Densidades a posteriori, traços e ACFs para a componente $T_{D}$ (Óbito) do ajuste do modelo fixo com $\left(J_{0}, J_{1}, J_{2}\right)=(2,2,2)$ segundo a cópula de Clayton aos dados de IRC. . . . . . . . . . . . . . . . . . . 145

D.6 Densidades a posteriori, traços e ACFs para a componente $T_{E}$ (Progressão) do ajuste do modelo fixo com $\left(J_{0}, J_{1}, J_{2}\right)=(2,2,2)$ segundo a cópula de Clayton aos dados de IRC. . . . . . . . . . . . . . . . . . . . . . 146

D.7 Densidades a posteriori, traços e ACFs para a componente $T_{G}$ (Óbito após progressão) do ajuste do modelo fixo com $\left(J_{0}, J_{1}, J_{2}\right)=(2,2,2)$ segundo a cópula de Clayton aos dados de IRC . . . . . . . . . . . . . . . . . . . 147

D.8 Densidades a posteriori, traços e ACFs para a componente logito (Progressão) do ajuste do modelo fixo com $\left(J_{0}, J_{1}, J_{2}\right)=(2,2,2)$ segundo a cópula de Clayton aos dados de IRC. . . . . . . . . . . . . . . . . . . . . . . 148 
D.9 Densidades a posterioris, traços e ACFs para a componente $T_{D}$ (Óbito) do ajuste do modelo misto com $\left(J_{0}, J_{1}, J_{2}\right)=(1,1,1)$ segundo a cópula de Clayton aos dados de IRC. . . . . . . . . . . . . . . . . . . . . . . . . . . . 149

D.10 Densidades a posteriori, traços e ACFs para a componente $T_{E}$ (Progressão) do ajuste do modelo misto com $\left(J_{0}, J_{1}, J_{2}\right)=(1,1,1)$ segundo a cópula de Clayton aos dados de IRC. . . . . . . . . . . . . . . . . . . . . 150

D.11 Densidades a posteriori, traços e ACFs para a componente $T_{G}$ (Óbito após progressão) do ajuste do modelo misto com $\left(J_{0}, J_{1}, J_{2}\right)=(1,1,1)$ segundo a cópula de Clayton aos dados de IRC. . . . . . . . . . . . . . . . . . . . . 151

D.12 Densidades a posteriori, traços e ACFs para a componente logito (Progressão) do ajuste do modelo misto com $\left(J_{0}, J_{1}, J_{2}\right)=(1,1,1)$ segundo a cópula de Clayton aos dados de IRC. . . . . . . . . . . . . . . . . . . . . . 152 


\section{Lista de Tabelas}

4.1 Valores dos parâmetros utilizados na geração dos conjuntos de dados. . . . . . 64

5.1 Descrição das covariáveis e eventos de interesse nos dados de TMO. . . . . . 74

5.2 Valores LPML, BIC DIC para o modelo fixo sob a cópula de Clayton ajustados aos dados de TMO. . . . . . . . . . . . . . . . . . . . . . 75

5.3 Ajuste modelagem fixa aos dados de TMO com $\left(J_{0}, J_{1}, J_{2}\right)=(15,15,15)$. . . 77

5.4 Descrição das covariáveis e eventos de interesse do estudo de IRC. . . . . . . 79

5.5 Distribuição do número de pacientes n(\%) com IRC pelas covariáveis segundo os eventos de interesse. . . . . . . . . . . . . . . . . . . . . . . . 80

5.6 Valores LPML, BIC DIC para os modelos fixos e mistos segundo a cópula de Clayton ajustados aos dados de IRC . . . . . . . . . . . . . . . . . . . . . . 82

5.7 Ajuste modelo fixo aos dados de IRC com as partições para as funções de risco basal $\left(J_{0}, J_{1}, J_{2}\right)=(2,2,2)$. Componentes: Logito e Modelo $T_{D}$. . . . . . . . 84

5.8 Ajuste modelo fixo aos dados de IRC com as partições para as funções de risco basal $\left(J_{0}, J_{1}, J_{2}\right)=(2,2,2)$. Componentes: Modelos $T_{E}$ e $T_{G}$. . . . . . . 85

5.9 Ajuste modelo misto aos dados de IRC com as partições para as funções de risco basal $\left(J_{0}, J_{1}, J_{2}\right)=(1,1,1) \ldots \ldots \ldots \ldots$. . . . . . . . . . 87

C.1 Resumo de 200 replicas com $\theta=1$, censura: $10 \%$ e $\left(J_{0}, J_{1}, J_{2}\right)=(3,3,3)$ segundo a cópula de Clayton. Modelo misto. (Valor: Verdadeiro valor do parâmetro, Est: média das médias a posteriori, SD: média dos desvios padrão a posteriori, RMSE: média das raízes quadradas do erro quadrático médio, CP: probabilidade de cobertura do HPD de 95\%) . . . . . . . . . . . . . . . 116 
C.2 Resumo de 200 replicas com $\theta=1$, censura: $45 \%$ e $\left(J_{0}, J_{1}, J_{2}\right)=(3,3,3)$ segundo a cópula de Clayton. Modelo misto. (Valor: Verdadeiro valor do parâmetro, Est: média das médias a posteriori, SD: média dos desvios padrão a posteriori, RMSE: média das raízes quadradas do erro quadrático médio, CP: probabilidade de cobertura do HPD de 95\%) . . . . . . . . . . . . . . . . . 117

C.3 Resumo de 200 replicas com $\theta=1$, censura: $70 \%$ e $\left(J_{0}, J_{1}, J_{2}\right)=(3,3,3)$ segundo a cópula de Clayton. Modelo misto. (Valor: Verdadeiro valor do parâmetro, Est: média das médias a posteriori, SD: média dos desvios padrão a posteriori, RMSE: média das raízes quadradas do erro quadrático médio, CP: probabilidade de cobertura do HPD de 95\%) . . . . . . . . . . . . . . . 118

C.4 Resumo de 200 replicas com $\theta=2$, censura: $10 \%$ e $\left(J_{0}, J_{1}, J_{2}\right)=(3,3,3)$ segundo a cópula de Clayton. Modelo misto. (Valor: Verdadeiro valor do parâmetro, Est: média das médias a posteriori, SD: média dos desvios padrão a posteriori, RMSE: média das raízes quadradas do erro quadrático médio, CP: probabilidade de cobertura do HPD de 95\%) . . . . . . . . . . . . . . . . . . 119

C.5 Resumo de 200 replicas com $\theta=2$, censura: $45 \%$ e $\left(J_{0}, J_{1}, J_{2}\right)=(3,3,3)$ segundo a cópula de Clayton. Modelo misto. (Valor: Verdadeiro valor do parâmetro, Est: média das médias a posteriori, SD: média dos desvios padrão a posteriori, RMSE: média das raízes quadradas do erro quadrático médio, CP: probabilidade de cobertura do HPD de 95\%) . . . . . . . . . . . . . . . . 120

C.6 Resumo de 200 replicas com $\theta=2$, censura: $70 \%$ e $\left(J_{0}, J_{1}, J_{2}\right)=(3,3,3)$ segundo a cópula de Clayton. Modelo misto. (Valor: Verdadeiro valor do parâmetro, Est: média das médias a posteriori, SD: média dos desvios padrão a posteriori, RMSE: média das raízes quadradas do erro quadrático médio, CP: probabilidade de cobertura do HPD de 95\%) . . . . . . . . . . . . . . . . . . 121

C.7 Resumo de 200 replicas de tamanho $N=1000$, com $\theta=1$, censura: $10 \%$ $e\left(J_{0}, J_{1}, J_{2}\right)=(3,3,3)$ segundo a cópula de Clayton. Modelo misto. Dados gerados com distribuição Weibull.(Valor: Verdadeiro valor do parâmetro, Est: média das médias a posteriori, SD: média dos desvios padrão a posteriori, RMSE: média das raízes quadradas do erro quadrático médio, CP: probabilidade de cobertura do HPD de 95\%) . . . . . . . . . . . . . . . . . . . . . . 122

C.8 Resumo de 200 replicas de tamanho $N=500$, com $\theta=1$, censura: $45 \%$ e $\left(J_{0}, J_{1}, J_{2}\right)=(3,3,3)$ segundo a cópula de Clayton. Modelo misto. Dados gerados com distribuição Weibull.(Valor: Verdadeiro valor do parâmetro, Est: média das médias a posteriori, SD: média dos desvios padrão a posteriori, RMSE: média das raízes quadradas do erro quadrático médio, CP: probabilidade de cobertura do HPD de 95\%) . . . . . . . . . . . . . . . . . . . . . . . 123 
C.9 Resumo de 200 replicas $\operatorname{com} \theta=1$, censura: $10 \%$ e $\left(J_{0}, J_{1}, J_{2}\right)=(3,3,3)$ segundo a cópula de Clayton. Modelo fixo. (Valor: Verdadeiro valor do parâmetro, Est: média das médias a posteriori, SD: média dos desvios padrão a posteriori, RMSE: média das raízes quadradas do erro quadrático médio, CP: probabilidade de cobertura do HPD de 95\%) . . . . . . . . . . . . . . . . . . . . . . . . 124

C.10 Resumo de 200 replicas com $\theta=1$, censura: $45 \%$ e $\left(J_{0}, J_{1}, J_{2}\right)=(3,3,3)$ segundo a cópula de Clayton. Modelo fixo. (Valor: Verdadeiro valor do parâmetro, Est: média das médias a posteriori, SD: média dos desvios padrão a posteriori, RMSE: média das raízes quadradas do erro quadrático médio, CP: probabilidade de cobertura do HPD de 95\%) . . . . . . . . . . . . . . . . . . . 125

C.11 Resumo de 200 replicas com $\theta=1$, censura: $70 \%$ e $\left(J_{0}, J_{1}, J_{2}\right)=(3,3,3)$ segundo a cópula de Clayton. Modelo fixo. (Valor: Verdadeiro valor do parâmetro, Est: média das médias a posteriori, SD: média dos desvios padrão a posteriori, RMSE: média das raízes quadradas do erro quadrático médio, CP: probabilidade de cobertura do HPD de 95\%) . . . . . . . . . . . . . . . . . 126 


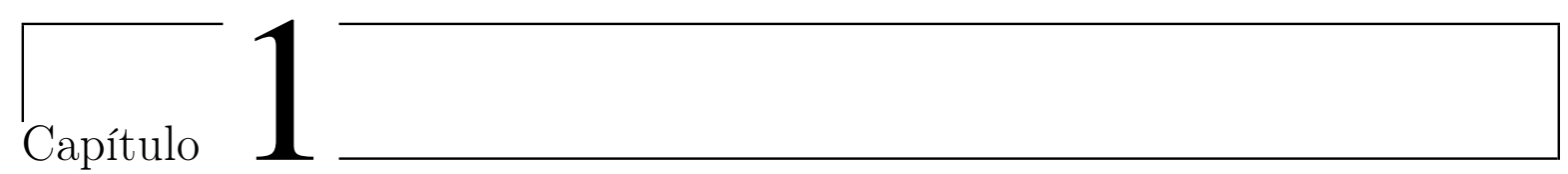

\section{Introdução}

Quando dados de sobrevivência são objeto de estudo, principalmente em pesquisa clínica que envolve seguimento de pacientes, em geral o interesse é centrado em identificar fatores prognósticos, analisar o efeito de um certo tratamento ou medir a influência de certas características sobre o tempo até a ocorrência de um evento definido, seja este óbito, transplante, uma progressão a um nível específico da condição médica estudada (metástase, por exemplo, em estudos oncológicos), etc. Em estudos sobre o vírus da imunodeficiência humana, os pacientes infectados são monitorados, por exemplo, até o desenvolvimento da síndrome da imunodeficiência adquirida (SIDA). No entanto, observa-se que em muitas situações os pacientes podem experimentar mais de um evento que pode ser de interesse, principalmente quando se trata de uma doença crônica. Um exemplo é um estudo sobre Insuficiência Cardíaca Congestiva (ICC), em que o interesse centra-se no tempo até o transplante do coração e/ou óbito. Outras situações similares a serem citadas são:

- o estudo da insuficiência renal crônica (IRC), (Patiño, 2012) que considera tratamento de hemodiálise e diálise peritoneal e em que o interesse dos pesquisadores concentra-se no tempo até uma progressão da doença e/ou óbito;

- o estudo de câncer colorretal analisado por Zeng et al. (2012) e por Zhang et al. (2014) em que o interesse também é a progressão da doença e/ou óbito; e

- o estudo de câncer pancreático tratado por Lee et al. (2015) e Lee et al. (2016). Neste caso, os pesquisadores estão interessados nos tempos até a readmissão e/ou óbito.

Uma sugestão para análise nos casos anteriores seria utilizar modelos de riscos competitivos, em que se observa o mínimo dos tempos independentes $T_{1}, T_{2}, \ldots, T_{k}, k \geq 2$, e $T_{i}$ denota o tempo de falha devido à $i$-ésima causa ${ }^{1}$. Porém, observe que nos eventos citados há interesse em estudar dois eventos, sendo um deles o óbito e um outro que pode (ou não) ocorrer antes do óbito. Em geral, o óbito é chamado de evento terminal e o outro evento (por exemplo, a progressão) é conhecido como evento intermediário

\footnotetext{
${ }^{1}$ Detalhes de modelos com estrutura de riscos competitivos podem ser consultados, por exemplo, em Klein e Moeschberger (2003) ou Kalbfleisch e Prentice (2002).
} 
ou não terminal. Quando o interesse do estudo está em avaliar esses dois eventos, um terminal e outro intermediário, a análise adontando-se uma abordagem de riscos competitivos pode levar a estimativas viesadas. Isto acontece porque as ocorrências destes dois eventos podem estar associadas devido ao fato que a ocorrência do evento terminal pode impedir a observação do evento intermediário, mas não vice-versa. Este tipo de situação foi denominada por Fine et al. (2001) como riscos semicompetitivos e é cada vez mais comum em ensaios clínicos.

As principais características dos dados com riscos semicompetitivos são: $(i)$ a existência de dados censurados, comuns em dados de sobrevivência e (ii) o fato de que se tem um par de variáveis aleatórias com a restrição que uma é estatísticamente menor que a outra (a progressão necessariamente ocorre antes do óbito) ou uma delas não é observada, o que leva à incorporação da provável dependência entre o tempo até a observação do evento intermediário e o tempo até a observação do evento terminal.

A estrutura de riscos semicompetitivos está sendo cada vez mais aceita, e tem recebido bastante atenção nos últimos anos.

\subsection{Revisão da literatura}

Partindo da ideia de que os eventos, intermediário e terminal, estão associados, Fine et al. (2001) propõem incorporar a dependência entre os tempos observados com base em um modelo de fragilidade gama no quadrante superior (considerando um plano cartesiano, $X \leq Y$ ), gerando assim uma função de sobrevivência bivariada, que no caso sem covariáveis corresponde à cópula de Clayton, pertencente à família de cópulas Arquimedianas. Pela dependência entre os eventos, não podem ser usados os procedimentos habituais para a estimação das funções de sobrevivência marginais, que podem ser usadas para a estimação da função de sobrevivência bivariada por meio de cópulas. Deste modo, Fine et al. (2001) propõem um estimador não paramétrico para as funções de sobrevivência marginais e derivam suas propriedades assintóticas.

A partir do trabalho Oakes (1989), que trata modelos de sobrevivência bivariados induzidos por fragilidades, pode-se verificar que qualquer modelo de fragilidade multiplicativo para dados de sobrevivência bivariados pode ser expresso por uma representação via cópula Arquimediana (veja, por exemplo, Romeo (2005)). Com base nessa ideia, Lakhal et al. (2008) encontram uma equação de estimação para o parâmetro de dependência da cópula, e posteriormente derivam estimadores das funções de sobrevivência conjunta e marginais. Para as funções marginais, usam estimadores cópula-gráfico. Esta abordagem clássica é interessante dado que incorpora de forma conveniente a dependência entre os dois tempos observados.

Posteriormente, Xu et al. (2010), apresentam um modelo de regressão chamado doença-morte (Illness-death) que inclui a fragilidade gama como efeito aleatório multiplicativo. Nesta modelagem, o estudo dos eventos intermediário e terminal é feito com base em um processo de três estados e o interesse é modelar as funções de transição 
ou risco entre os estados. Considerando que a observação de outras variáveis, além dos tempos até a ocorrência dos eventos, é uma situação clássica bastante comum em análise de sobrevivência, uma vantagem deste processo é que, além da inclusão da fragilidade nas funções de transição, também podem ser incorporadas covariáveis às funções de risco condicionadas ao efeito aleatório usando um modelo de regressão semelhante ao modelo de riscos proporcionais de Cox (1972). Nesta proposta, a estimação dos parâmetros é feita por máxima verossimilhança. Os autores apresentam um algoritmo para a aproximação numérica, e discutem as propriedades assintóticas.

No caso do estudo de câncer colorretal, Zeng et al. (2012) usam também um modelo de fragilidade para determinar o efeito do tratamento, diferente do modelo doençamorte. Zeng et al. (2012) observam novas variáveis após a ocorrência do evento intermediário, além de uma possível mudança de tratamento (de placebo ao tratamento objeto do estudo), dado que pode ter havido uma piora clínica do paciente considerando que o evento intermediário ocorreu. Modelos de regressão que permitem a inclusão dessa nova informação quando o evento intermediário ocorre são bastate interessantes do ponto de vista aplicado. Para a estimação dos parâmetros, Zeng et al. (2012) desenvolvem um algoritmo EM para obter estimativas de máxima verossimilhança. Posteriormente e motivados pelo trabalho de Zeng et al. (2012), a abordagem bayesiana é aplicada aos dados de câncer colorretal por Zhang et al. (2014), que estudam e desenvolvem um algoritmo do tipo amostrador de Gibbs usando a técnica de Gibbs colapsado modificado (Lui, 1994) e rejeição adaptativa (Gilks e Wild, 1992).

Lee et al. (2015) também propõem uma análise de regressão bayesiana semiparamétrica com o propósito de fazer inferência sobre os parâmetros da regressão, caracterizar a dependência entre os eventos de interesse e fazer predição dado um perfil de covariáveis. O modelo é baseado no processo doença-morte com fragilidade gama descrito em $\mathrm{Xu}$ et al. (2010) cuja estimação é feita pelo algoritmo de Metropolis-Hastings-Green. O modelo é aplicado em pacientes com câncer pancreático observados entre 2005 e 2008 em centros médicos dos Estados Unidos. Aqui, os eventos de interesse são a readmissão dos pacientes diagnosticados como evento intermediário e o óbito como evento terminal.

Continuando com o estudo da qualidade da assistência para pacientes com câncer pancreático, Lee et al. (2016) propõem uma extensão do seu modelo (Lee et al., 2015). A nova modelagem trata os dados com riscos semicompetitivos quando há grupos correlacionados e, para isto, eles usam um modelo hierárquico que permite especificações paramétricas e não paramétricas.

Outros autores como Peng e Fine (2007), Hsieh et al. (2008), Lakhal et al. (2008), Hsieh e Huang (2012), e Chen (2012) também abordam os riscos semicompetitivos usando cópulas da família Arquimedianas. No entanto, a estimação nestes casos é estudada sob a abordagem clássica. 


\subsection{Motivação}

A motivação deste trabalho surgiu de um conjunto de dados de pacientes na Colômbia diagnosticados com insuficiência renal crônica (IRC) nos estágios mais severos da doença (ESRD End stage renal disease ${ }^{2}$ ). Atualmente, a IRC é um problema de ordem mundial e, segundo Martín-Cleary e Ortiz (2014), o número de mortes por esta causa tem aumentado na última década. Em particular, na Colômbia a incidência da IRC vem crescendo consideravelmente (Gómez, 2006). De acordo com Cuenta del Alto Costo (2014), para 2013 foram reportados 975.479 casos, sendo esta uma doença de alto custo, o que gera grande impacto no sistema de saúde colombiano. Dessa forma, pesquisas associadas à IRC são de grande interesse para as instituições gestoras de saúde.

Estes pacientes foram observados entre 2009 e 2011 desde o início do tratamento com diálise (peritoneal ou hemodiálise) até óbito, ocorrendo ou não uma progressão da doença, que foi determinada quando há uma mudança no quadro clínico devido a altos níveis de fósforo inorgânico no sangue. É claro que, no decorrer do período de observação, o paciente podia não apresentar nenhum dos eventos até o fim do estudo, ou seja, ser censurado ${ }^{3}$. Além disso, foram registradas as covariáveis sexo, idade no inicio do tratamento e cidade onde o paciente foi tratado.

Uma análise preliminar destes dados sob uma abordagem clássica é apresentada em Patiño (2012). Segundo os resultados obtidos, a cidade na qual o paciente foi tratado teve um efeito significativo no tempo de sobrevida tanto para a progressão da doença como para o óbito. Esta é então uma motivação para se considerar um modelo estendido incluindo um efeito aleatório, que associe pacientes tratados num mesmo local a um mesmo grupo (Zeng e Karim, 1991).

\subsection{Objetivos}

Motivados principalmente pelos dados de IRC descritos, o objetivo principal deste trabalho é propor um modelo para dados de sobrevivência com riscos semicompetitivos e mudança de tratamento após a ocorrência do evento intermediário, sob uma ótica bayesiana e usando cópulas da família Arquimediana, com ênfase na cópula de Clayton, para incorporar a dependência entre os tempos até o evento intermediário e o evento terminal.

Em seguida, pretende-se incluir no modelo um efeito aleatório que possa incorporar as possíveis dependências entre as observações vindas de um mesmo centro de tratamento.

\footnotetext{
${ }^{2}$ A classificação dos estágios da IRC pode ser consultada na página da Clínica Renal Colombiana no seguinte link http://www.clinicarenal.org/clinicarenal.org/articulos/articulo-33.html

${ }^{3}$ A censura é uma característica comum aos dados de sobrevivência em que há uma observação parcial do tempo de falha. Para mais detalhes veja Klein e Moeschberger (2003), Colosimo e Ruiz (2006) e Kalbfleisch e Prentice (2002). Neste trabalho somente é considerada censura à direita, sendo que a ocorrência do evento de interesse encontra-se à direita do tempo registrado.
} 
Mais detalhadamente, pode-se mencionar os seguintes objetivos específicos:

1. Propor e desenvolver um modelo bayesiano utilizando a cópula de Clayton incluindo mudança de tratamento, seguindo a proposta de Zhang et al. (2014).

2. Implementar a modelagem proposta no item 1 utilizando procedimentos de simulação Monte Carlo via cadeias de Markov (MCMC) para formular algoritmos do tipo amostrador de Gibbs (Casella e George, 1992).

3. Estender o modelo do item 1 com a inclusão de efeitos aleatórios e implementar o modelo estendido.

4. Modificar os modelos estudados nos itens 1 e 3 considerando diferentes cópulas como Frank e Gumbel, e implementá-los.

5. Realizar estudos de simulação apropriados para avaliar o desempenho da modelagem proposta.

6. Aplicar a modelagem proposta a conjuntos de dados reais.

7. Implementar critérios de avaliação para a modelagem proposta como o logaritmo da pseudoverossimilhança marginal (LPML) conforme discutido em Chen et al. (2000), o criterio de informação bayesiano (BIC) introduzido por Schwarz (1978) e considerado como em Sahu e Dey (2000) e o criterio de informação de desviância (DIC) como apresentado em Spiegelhalter et al. (2002).

\subsection{Contribuições}

A principal contribuição desta tese é uma extensão de Zhang et al. (2014) para dados de sobrevivência com riscos semicompetitivos sob uma abordagem bayesiana nos seguintes aspectos:

- Modelamento da dependência dos eventos de interesse por meio de cópulas da família Arquimediana. Pretende-se com isso flexibilizar o modelo a partir de diferentes formas da estrutura de dependência obtendo um modelo marginal. O modelo de fragilidade de Zhang et al. (2014) é um modelo condicional (Goethals et al., 2008).

- Inclusão de um efeito aleatório para lidar com a situação de dados agrupados, como por exemplo, indivíduos observados em um mesmo centro de tratamento.

\subsection{Organização do trabalho}

O trabalho está organizado em seis capítulos conforme detalhado a seguir.

No Capítulo 2 é apresentada a linguagem básica associada à estrutura de riscos semicompetitivos proposta por Fine et al. (2001). Também é feita uma breve descrição 
das cópulas Arquimedianas e a estrutura de dependência. Em seguida, no Capítulo 3, é descrita a modelagem proposta e sua componente teórica bayesiana; alguns aspectos deste capítulo estão descritos nos dois primeiros Apêndices, como a prova do teorema que garante que a distribuição a posteriori segundo a cópula de Clayton é própria, disponível no Apêndice B. No Capítulo 4 são tratados todos os aspectos computacionais da modelagem, assim como simulações para avaliar o desempenho da mesma. A seguir, no Capítulo 5 é exibida a aplicação da modelagem ao conjunto de dados reais de pacientes com IRC tratados na Colômbia e também aos dados de transplante de médula óssea (TMO) da Sociedade Européia para Sangue e Transplante de Medula (EBMT). Finalmente, no Capítulo 6 são discutidas algumas conclusões obtidas neste trabalho. Também são analisadas as vantagens e desvantagens da metodologia proposta e perspectivas de pesquisa futura. 


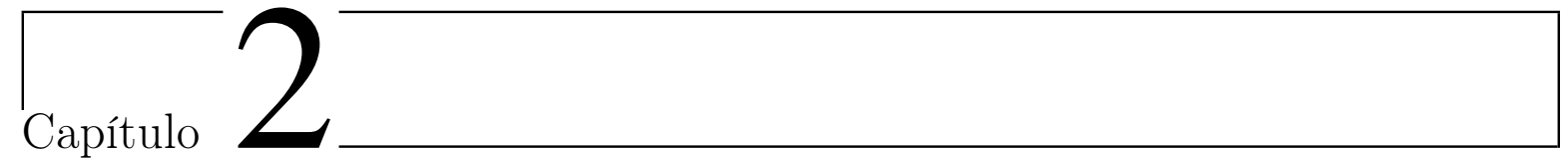

\section{Conceitos preliminares}

Neste capítulo são apresentadas as principais ferramentas que serão utilizadas ao longo deste trabalho. Inicialmente é discutida a estrutura dos riscos semicompetitivos. Em seguida é feita uma revisão sobre cópulas Arquimedianas para dados de sobrevivência bivariados e sua estrutura de dependência. Finalmente, é apresentada uma possível análise descritiva para dados com riscos semicompetitivos, conforme apresentado em Patiño (2012).

\subsection{Riscos semicompetitivos}

Os riscos semicompetitivos surgem como uma variação do problema dos riscos competitivos em que, como indicam Varadhan et al. (2014), são envolvidos dois tipos de eventos: um intermediário e outro terminal. Tipicamente, o foco dos estudos centra-se no evento intermediário (Lakhal et al., 2008), porém, a ocorrência deste evento pode ser impedida pelo evento terminal, mas o evento intermediário não impede a ocorrência do terminal.

Para o caso de riscos semicompetitivos em que dois eventos são de interesse, considerase a função de sobrevivência bivariada

$$
S\left(t_{1}, t_{2}\right)=\mathrm{P}\left(T_{1}>t_{1}, T_{2}>t_{2}\right) ; \quad 0 \leq t_{1} \leq t_{2},
$$

em que $T_{1}$ denota o tempo até a ocorrência do evento intermediário (progressão, por exemplo) e $T_{2}$ o tempo até a ocorrência do evento terminal (por exemplo, óbito) ${ }^{1}$.

Os riscos semicompetitivos possuem as seguintes características:

- Trata-se do estudo de um vetor aleatório bivariado $\left(T_{1}, T_{2}\right)$.

- A ocorrência do evento intermediário nem sempre é observada, pois no caso em que o evento terminal ocorre antes do intermediário $\left(T_{2}<T_{1}\right)$, o evento intermediário

\footnotetext{
${ }^{1}$ Se $t_{1}>t_{2}$, uma estratégia para a especificação da função de densidade conjunta de $\left(T_{1}, T_{2}\right)$ é considerar $T_{1}=\infty$. Detalhes podem ser consultados em Wang (2003), Xu et al. (2010) e Lee et al. (2015)
} 
já não pode ser observado, visto que sua ocorrência foi impedida pelo evento terminal.

- $T_{1}$ e $T_{2}$ são variáveis aleatórias, positivas e observadas conjuntamente, tais que $T_{1} \leq T_{2}$.

- Em consequência do item anterior, espera-se que os tempos de ambos os eventos $T_{1}$ e $T_{2}$ estejam correlacionados e, portanto, a função de sobrevivência bivariada (2.1) não pode ser tratada como o produto de suas funções marginais $S\left(t_{1}\right)$ e $S\left(t_{2}\right)$.

Além das características do processo associado à estrutura de riscos semicompetitivos, é muito comum que as observações estejam sujeitas à censura.

\subsection{Cópulas e estrutura de dependência}

A seguir serão discutidas cópulas bivariadas, considerando que, a função de sobrevivência bivariada é uma das componentes da modelagem objeto do estudo neste trabalho.

A cópula pode ser definida como uma função que acopla as funções de distribuição marginais $F(x)$ e $G(y)$ das variáveis aleatórias contínuas $X$ e $Y$, respectivamente, numa função de distribuição bivariada $H(x, y)$ por meio da relação $H(x, y)=\mathcal{C}_{\theta}(F(x), G(y))$. Um resultado que estabelece a relação entre cópulas e funções bivariadas é o Teorema de Sklar (Sklar, 1959). Detalhes podem ser consultados em Nelsen (2000).

Teorema de Sklar. Seja $H$ uma função de distribuição bidimensional com funções de distribuição marginais $F$ e $G$. Então, existe uma cópula $\mathcal{C}$ tal que $H(x, y)=$ $\mathcal{C}(F(x), G(y))$. Reciprocamente, para quaisquer funções de distribuição $F$ e $G$ e qualquer cópula $\mathcal{C}$, a função $H$ definida acima é uma função de distribuição bidimensional com marginais $F$ e $G$. Além disso, se $F$ e $G$ são contínuas, $\mathcal{C}$ é única.

O Teorema de Sklar mostra que as cópulas são funções que ligam funções de distribuição multivariadas com suas funções de distribuição marginais. Observe também que as cópulas são funções de distribuição multivariadas compostas por marginais uniformes no intervalo $[0,1]$ (Romeo, 2005).

Neste trabalho, serão consideradas cópulas da família Arquimediana definida a seguir e comumente referenciada e utilizada na literatura para modelos de sobrevivência bivariados e modelos multiplicativos de fragilidade, veja por exemplo Romeo et al. (2006), Lakhal et al. (2008) e Xu et al. (2010) entre outros.

Definição 1 A cópula $\mathcal{C}_{\theta}(\cdot)$ é dita Arquimediana se

$$
\mathcal{C}_{\theta}(u, v)=\phi_{\theta}^{-1}\left\{\phi_{\theta}(u)+\phi_{\theta}(v)\right\}, \quad 0 \leq u \leq 1,0 \leq v \leq 1 \quad e \quad \theta \in \Theta .
$$

Em que $\phi_{\theta}(\cdot):[0,1] \rightarrow[0, \infty)$ é uma função contínua, estritamente decrescente e convexa, tal que $\phi_{\theta}(1)=0, \phi_{\theta}^{\prime}(\cdot)<0$ e $\phi_{\theta}^{\prime \prime}(\cdot) \geq 0$. A função $\phi_{\theta}(\cdot)$ é chamada função geradora da cópula. 
Como observado na definição anterior, esta família de cópulas é indexada pelo parâmetro $\theta$. O parâmetro $\theta$ não tem interpretação própria, porém, é útil para medir a associação entre as variáveis aleatórias da cópula por meio de medidas de concordância, como o tau de Kendall $\left(\tau_{\theta}\right)$. Esta medida será usada neste trabalho por ser de ampla utilização e fácil interpretação. Outras medidas de dependência podem ser encontradas em Nelsen (2000) (p. 180).

No contexto dos riscos semicompetitivos, o parâmetro $\theta$ da cópula está associado à dependência entre os tempos dos eventos de interesse, intermediário e final. Segundo Lakhal et al. (2008), este parâmetro é útil para medir a capacidade do evento intermediário predizer a ocorrência do evento terminal.

\subsubsection{Tau de Kendall}

Uma forma de quantificar a associação entre os tempos dos eventos intermediário $T_{1}$ e final $T_{2}$ é por meio do coeficiente de concordância $\tau_{\theta}$ de Kendall, uma medida não paramétrica de dependência global que quantifica a diferença entre a probabilidade de um par de variáveis aleatórias ser concordante ou discordante.

Um par de variáveis é concordante se grandes valores de uma variável tendem a estar associados com grandes valores da outra variável ou pequenos valores de uma variável tendem a estar associados com pequenos valores da outra variável. Em outras palavras, dados os pares observados $\left(x_{1}, y_{1}\right)$ e $\left(x_{2}, y_{2}\right)$ das variáveis aleatórias $\left(X_{1}, Y_{1}\right)$ e $\left(X_{2}, Y_{2}\right)$, eles são concordantes se $x_{i}>x_{j}$ e $y_{i}>y_{j}$ ou se $x_{i}<x_{j}$ e $y_{i}<y_{j}$, e eles são discordantes se $x_{i}>x_{j}$ e $y_{i}<y_{j}$ ou se $x_{i}<x_{j}$ e $y_{i}>y_{j}$. Pode-se ainda verificar que os pares são concordantes se $\left(x_{i}-x_{j}\right)\left(y_{i}-y_{j}\right)>0$ e discordantes se $\left(x_{i}-x_{j}\right)\left(y_{i}-y_{j}\right)<0$.

O coeficiente $\tau$ de Kendall é definido com base nessa ideia, conforme a definição a seguir (Nelsen, 2000).

Definição 2 Sejam $\left(X_{1}, Y_{1}\right)$ e $\left(X_{2}, Y_{2}\right)$ duas réplicas independentes de um par $(X, Y)$ de variáveis aleatórias quaisquer. $O$ coeficiente $\tau_{\theta}$ de Kendall é definido por

$$
\begin{aligned}
& \tau_{\theta}= \operatorname{Pr}\left\{\left(X_{1}-X_{2}\right)\left(Y_{1}-Y_{2}\right)>0\right\}-\operatorname{Pr}\left\{\left(X_{1}-X_{2}\right)\left(Y_{1}-Y_{2}\right)<0\right\} \\
&= \mathbb{E}\left\{\operatorname{sgn}\left(\left(X_{1}-X_{2}\right)\left(Y_{1}-Y_{2}\right)\right)\right\}, \\
& \text { em que } \operatorname{sgn}(\mathrm{x})= \begin{cases}-1, & \text { se } x<0, \\
0, & \text { se } x=0, \\
1, & \text { se } x>0 .\end{cases}
\end{aligned}
$$

Assumindo $X$ e $Y$ contínuas, vale o seguinte:

$$
\begin{aligned}
\tau_{\theta} & =\operatorname{Pr}\left\{\left(X_{1}-X_{2}\right)\left(Y_{1}-Y_{2}\right)>0\right\}-\operatorname{Pr}\left\{\left(X_{1}-X_{2}\right)\left(Y_{1}-Y_{2}\right)<0\right\} \\
& =4 \mathbb{E}\left\{S_{X Y}(X, Y)\right\}-1 \\
& =4 \iint S_{X Y}(x, y) f_{X Y}(x, y) d x d y-1,
\end{aligned}
$$


em que $f_{X Y}(x, y)$ e $S_{X Y}(x, y)$ representam a função densidade de probabilidade conjunta e a função de sobrevivência conjunta de $X$ e $Y$, respectivamente.

Se $S_{X Y}(x, y)$ é representada usando a cópula de sobrevivência bivariada $\mathcal{C}_{\theta}(u, v)$ gerada por $\phi_{\theta}(\cdot)$, então o coeficiente $\tau_{\theta}$ de Kendall, admite a seguinte expressão ${ }^{2}$ :

$$
\tau_{\theta}=4 \iint S_{X Y}(x, y) f_{X Y}(x, y) d x d y-1=4 \int_{0}^{1} \frac{\phi_{\theta}(u)}{\phi_{\theta}^{\prime}(u)} d u+1 .
$$

Nelsen (2000) (Cap. 4) apresenta a parametrização de 22 cópulas da família Arquimediana, entre elas as cópulas mais populares que serão abordadas neste trabalho: Clayton, Frank e Gumbel, conforme descrito nas seguintes subseções.

\subsubsection{Cópula de Clayton}

A cópula de Clayton é uma cópula Arquimediana simétrica, com função geradora $\phi(t)=\left(t^{\theta}-1\right) / \theta$. Seguindo a Definição 1, ela é dada por

$$
\mathcal{C}_{\theta}(u, v)=\left(u^{-\theta}+v^{-\theta}-1\right)^{-\frac{1}{\theta}} \quad \theta \in(0, \infty) .
$$

Observe que se $\theta \rightarrow 0, \mathcal{C}_{\theta}(u, v)=u v$, portanto, $\theta \rightarrow 0$ implica independência, enquanto que, $\theta \rightarrow \infty$ indica dependência positiva perfeita. O coeficiente tau de Kendall, associado a este parâmetro, avaliado em (2.2) é dado por $\tau_{\theta}=\frac{\theta}{\theta+2}$.

Assumindo $u=S_{1}\left(t_{1}\right)$ e $v=S_{2}\left(t_{2}\right)$, a função de sobrevivência conjunta de $\left(T_{1}, T_{2}\right)$ é expressa como

$$
S_{\theta}\left(t_{1}, t_{2}\right)=\left(\left[S_{1}\left(t_{1}\right)\right]^{-\theta}+\left[S_{2}\left(t_{2}\right)\right]^{-\theta}-1\right)^{-\frac{1}{\theta}} .
$$

Similarmente, a função densidade bivariada pode ser escrita como

$$
f_{\theta}\left(t_{1}, t_{2}\right)=c_{\theta}\left(S_{1}\left(t_{1}\right), S_{2}\left(t_{2}\right)\right) f_{1}\left(t_{1}\right) f_{2}\left(t_{2}\right),
$$

em que

$$
c_{\theta}\left(S_{1}\left(t_{1}\right), S_{2}\left(t_{2}\right)\right)=\left.\frac{\partial^{2} \mathcal{C}_{\theta}(u, v)}{\partial u \partial v}\right|_{u=S_{1}\left(t_{1}\right), v=S_{2}\left(t_{2}\right)} .
$$

Como

$$
\frac{\partial^{2} \mathcal{C}_{\theta}(u, v)}{\partial u \partial v}=(1+\theta)(u v)^{-\theta-1}\left(u^{-\theta}+v^{-\theta}-1\right)^{-\frac{1}{\theta}-2},
$$

segue que

$$
f_{\theta}\left(t_{1}, t_{2}\right)=\frac{(1+\theta) f_{1}\left(t_{1}\right) f_{2}\left(t_{2}\right)}{\left(S_{1}\left(t_{1}\right) S_{2}\left(t_{2}\right)\right)^{\theta+1}\left(\left[S_{1}\left(t_{1}\right)\right]^{-\theta}+\left[S_{1}\left(t_{1}\right)\right]^{-\theta}-1\right)^{\frac{1}{\theta}+2}} .
$$

A Figura 2.1 representa as funções distribuição e densidade para a cópula de Clayton. Conforme pode-se observar, a função densidade atinge valor máximo no extremo

\footnotetext{
${ }^{2}$ Detalhes da prova podem ser encontrados em Nelsen (2000), página 163.
} 

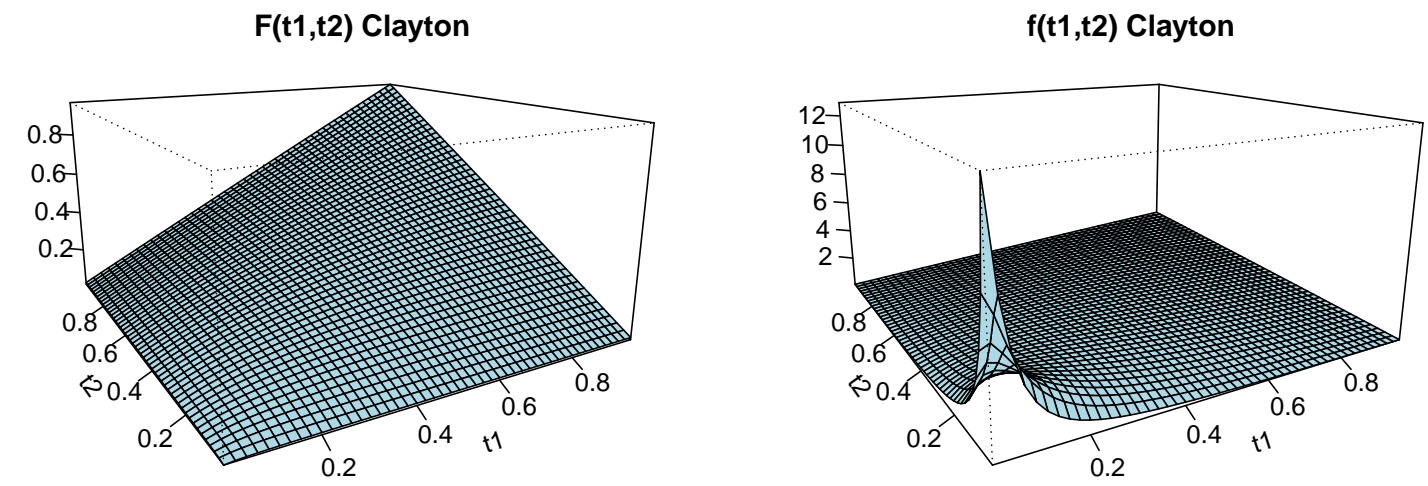

Figura 2.1: Função distribuição (esquerda) e função densidade (direita) da cópula de Clayton $\operatorname{com} \theta=1(\tau=0,333)$.

inferior do quadrado unitário indicando que a área de dependência das variáveis se encontra próxima ao ponto $(0,0)$.

Esta cópula está associada ao modelo multiplicativo de fragilidade gama (Yan (2007), Romeo (2005)).

\subsubsection{Cópula de Frank}

Esta cópula estudada por Frank (1979) é dada por

$$
\mathcal{C}_{\alpha}(u, v)=\log _{\alpha}\left(1+\frac{\left(\alpha^{u}-1\right)\left(\alpha^{v}-1\right)}{\alpha-1}\right), \quad \alpha \in(0,1)
$$

$\mathrm{O}$ caso de independência acontece quando $\alpha \rightarrow 1$. No entanto, ao considerar a reparametrização $\alpha=\mathrm{e}^{-\theta}$, com $\theta \neq 0$, é capturado todo o campo de variação da associação, tanto positiva como negativa. Assim, a cópula é definida como

$$
\mathcal{C}_{\theta}(u, v)=-\frac{1}{\theta} \ln \left(1+\frac{\left(\mathrm{e}^{-\theta u}-1\right)\left(\mathrm{e}^{-\theta v}-1\right)}{\mathrm{e}^{-\theta}-1}\right), \quad \theta \in \mathbb{R}
$$

De (2.5) tem-se que a função de sobrevivência conjunta de $\left(T_{1}, T_{2}\right)$ é dada por

$$
S_{\theta}\left(t_{1}, t_{2}\right)=-\frac{1}{\theta} \ln \left(1+\frac{\left(\mathrm{e}^{-\theta S_{1}\left(t_{1}\right)}-1\right)\left(\mathrm{e}^{-\theta S_{2}\left(t_{2}\right)}-1\right)}{\mathrm{e}^{-\theta}-1}\right) .
$$

Para a função densidade, tem-se os seguintes resultados

$$
\frac{\partial \mathcal{C}_{\theta}(u, v)}{\partial u}=\frac{\mathrm{e}^{-\theta(u+v)}-\mathrm{e}^{-\theta u}}{\mathrm{e}^{-\theta}+\mathrm{e}^{-\theta(u+v)}-\mathrm{e}^{-\theta u}-\mathrm{e}^{-\theta v}}=\frac{\mathrm{e}^{-\theta u}\left(\mathrm{e}^{-\theta v}-1\right)}{\mathrm{e}^{-\theta}-1+\left(\mathrm{e}^{-\theta u}-1\right)\left(\mathrm{e}^{-\theta v}-1\right)}
$$


e

$$
\frac{\partial^{2} \mathcal{C}_{\theta}(u, v)}{\partial u \partial v}=\frac{\theta\left(1-\mathrm{e}^{-\theta}\right) \mathrm{e}^{-\theta(u+v)}}{\left[\mathrm{e}^{-\theta}+\mathrm{e}^{-\theta(u+v)}-\mathrm{e}^{-\theta u}-\mathrm{e}^{-\theta v}\right]^{2}}=\frac{\theta\left(1-\mathrm{e}^{-\theta}\right) \mathrm{e}^{-\theta(u+v)}}{\left[\mathrm{e}^{-\theta}-1+\left(\mathrm{e}^{-\theta u}-1\right)\left(\mathrm{e}^{-\theta v}-1\right)\right]^{2}} .
$$

Finalmente, chega-se a

$$
f_{\theta}\left(t_{1}, t_{2}\right)=\frac{\theta\left(1-\mathrm{e}^{-\theta}\right) \mathrm{e}^{-\theta\left(S_{1}\left(t_{1}\right)+S_{2}\left(t_{2}\right)\right)} f_{1}\left(t_{1}\right) f_{2}\left(t_{2}\right)}{\left[\mathrm{e}^{-\theta}-1+\left(\mathrm{e}^{-\theta S_{1}\left(t_{1}\right)}-1\right)\left(\mathrm{e}^{-\theta S_{2}\left(t_{2}\right)}-1\right)\right]^{2}} .
$$

A Figura 2.2 apresenta as funções distribuição e densidade para a cópula de Frank. Nesta cópula, pode-se observar que a massa se distribui na diagonal entre $t_{1}$ e $t_{2}$ com valores máximos nos extremos.
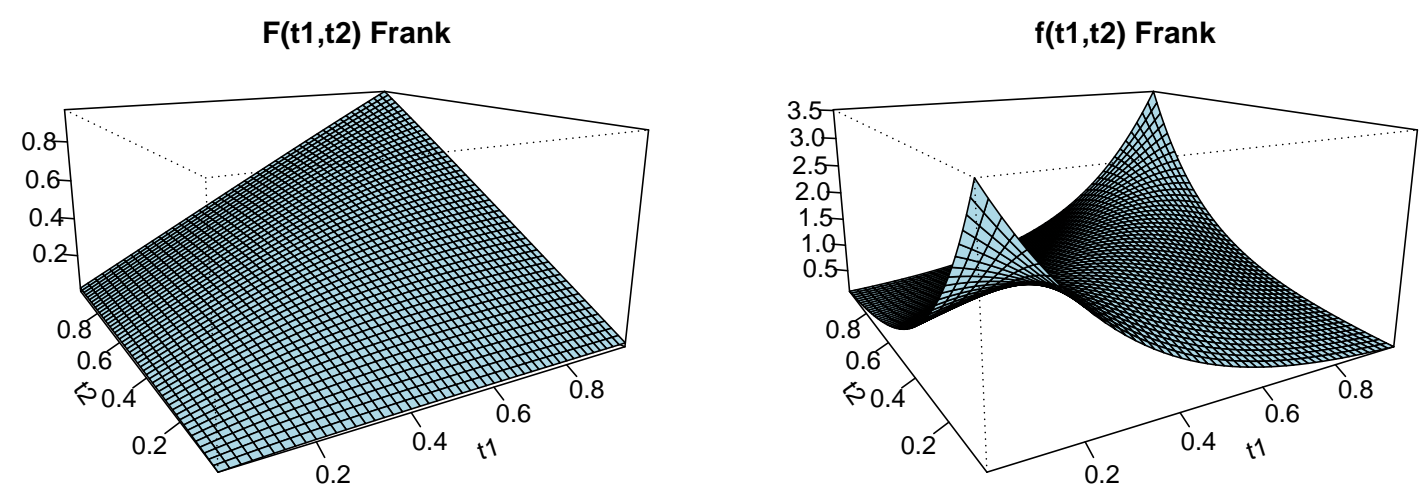

Figura 2.2: Função distribuição (esquerda) e função densidade (direita) da cópula de Frank $\operatorname{com} \theta=4(\tau \simeq 0,388)$.

De (2.2) pode ser verificado que o coeficiente tau de Kendall para a cópula de Frank é dado por

$$
\tau_{\theta}=1-\frac{4}{\theta}\left(1-\frac{1}{\theta} \int_{0}^{\theta} \frac{t}{\mathrm{e}^{t}-1} d t\right)
$$

em que $\frac{1}{\theta} \int_{0}^{\theta} \frac{t}{\mathrm{e}^{t}-1} d t$ corresponde à função Debye de primeira ordem. As funções Debye $D_{n}(x)$ são definidas como $D_{n}(x)=\frac{n}{x^{n}} \int_{0}^{x} \frac{t^{n}}{\mathrm{e}^{t}-1} d t$.

Como menciona Yan (2007) a cópula de Frank está associada a modelos de fragilidade das series log.

\subsubsection{Cópula de Gumbel ou fragilidade estável positiva}

Esta cópula é definida por

$$
\mathcal{C}_{\theta}(u, v)=\exp \left\{-\left[(-\ln u)^{\theta}+(-\ln v)^{\theta}\right]^{\frac{1}{\theta}}\right\} \quad \theta \in[1, \infty) .
$$


Note que, quando $\theta \rightarrow 1$, obtém-se o caso de independência. Segundo (2.2), o coeficiente tau de Kendall é dado por $\tau_{\theta}=1-\frac{1}{\theta}$.

Conforme (2.7), a função de sobrevivência bivariada de $\left(T_{1}, T_{2}\right)$ fica expressa por

$$
S_{\theta}\left(t_{1}, t_{2}\right)=\exp \left\{-\left[\left(-\ln S_{1}\left(t_{1}\right)\right)^{\theta}+\left(-\ln S_{2}\left(t_{2}\right)\right)^{\theta}\right]^{\frac{1}{\theta}}\right\} .
$$

Para a função densidade tem-se que

$$
\frac{\partial \mathcal{C}_{\theta}(u, v)}{\partial u}=\exp \left\{-\left[(-\ln u)^{\theta}+(-\ln v)^{\theta}\right]^{\frac{1}{\theta}}\right\}\left[(-\ln u)^{\theta}+(-\ln v)^{\theta}\right]^{\frac{1}{\theta}-1} \frac{(-\ln u)^{\theta-1}}{u}
$$

$\mathrm{e}$

$$
\begin{aligned}
\frac{\partial^{2} \mathcal{C}_{\theta}(u, v)}{\partial u \partial v} & =\frac{(-\ln u)^{\theta-1}}{u} \frac{(-\ln v)^{\theta-1}}{v} \exp \left\{-\left[(-\ln u)^{\theta}+(-\ln v)^{\theta}\right]^{\frac{1}{\theta}}\right\}\left[(-\ln u)^{\theta}+(-\ln v)^{\theta}\right]^{\frac{1}{\theta}-2} \\
& \times\left(\theta-1+\left[(-\ln u)^{\theta}+(-\ln v)^{\theta}\right]^{\frac{1}{\theta}}\right)
\end{aligned}
$$

obtendo-se finalmente

$$
\begin{aligned}
f_{\theta}\left(t_{1}, t_{2}\right) & =\frac{\left(-\ln S_{1}\left(t_{1}\right)\right)^{\theta-1}}{S_{1}\left(t_{1}\right)} \frac{\left(-\ln S_{2}\left(t_{2}\right)\right)^{\theta-1}}{S_{2}\left(t_{2}\right)} \\
& \times \exp \left\{-\left[\left(-\ln S_{1}\left(t_{1}\right)\right)^{\theta}+\left(-\ln S_{2}\left(t_{2}\right)\right)^{\theta}\right]^{\frac{1}{\theta}}\right\}\left[\left(-\ln S_{1}\left(t_{1}\right)\right)^{\theta}+\left(-\ln S_{2}\left(t_{2}\right)\right)^{\theta}\right]^{\frac{1}{\theta}-2} \\
& \times\left(\theta-1+\left[\left(-\ln S_{1}\left(t_{1}\right)\right)^{\theta}+\left(-\ln S_{2}\left(t_{2}\right)\right)^{\theta}\right]^{\frac{1}{\theta}}\right) f_{1}\left(t_{1}\right) f_{2}\left(t_{2}\right) .
\end{aligned}
$$

De acordo com a Figura 2.3, a massa para a cópula de Gumbel está concentrada nos extremos da diagonal principal, porém com maior concentração no extremo direito (coordenadas $(1,1)$ ) indicando uma forte associação nesta região.

$F(t 1, t 2)$ Gumbel

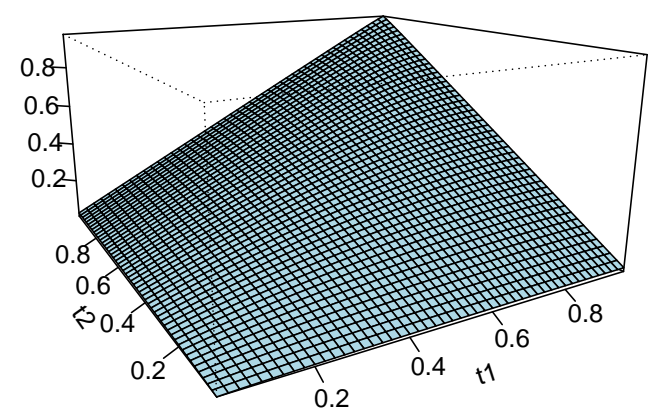

$f(t 1, t 2)$ Gumbel

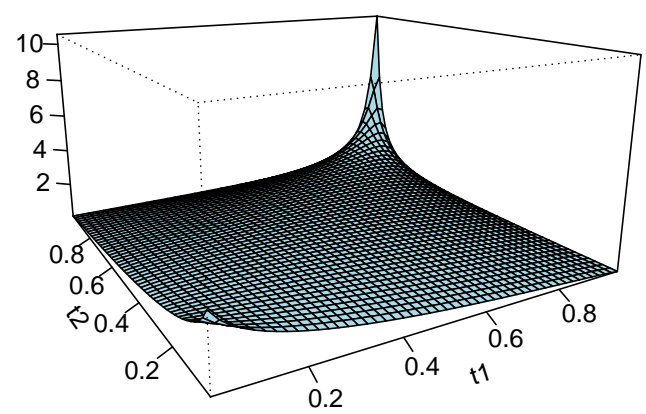

Figura 2.3: Função distribuição (esquerda) e função densidade (direita) da cópula de Gumbel $\operatorname{com} \theta=1,5(\tau=0,333)$. 


\subsection{Análise descritiva para dados com riscos semicom- petitivos}

Nesta seção é apresentada uma metodologia para a análise descritiva a dados com estrutura de riscos semicompetitivos. Vale ressaltar que o leitor não precisa fazer uma leitura desta seção para entender a modelagem proposta nesta tese no Capítulo 3. Como esse método para análise descritiva foi utilizado no Capítulo 5, optou-se por fazer uma breve descrição neste capítulo.

No caso de dados com estrutura de riscos semicompetitivos, a primeira ideia para uma análise descritiva seria a utilização do estimador de Kaplan-Meier para a análise do tempo até os eventos de interesse. Embora o estimador de Kaplan-Meier possa ser utilizado para a estimação da função de sobrevivência do evento terminal, para a estimação da função de sobrevivência do evento intermediário, este estimador não é apropriado, dada a possível dependência entre os eventos de interesse. Se for considerado que o óbito é também censura para o evento intermediário, a suposição de censura independente não é verificada e, portanto, não se pode utilizar técnicas de análise estatística que se baseiam nessa suposição. Nesse contexto, foram propostos vários estimadores: estimador não paramétrico de Fine et al. (2001), estimador auto-consistente de Jiang et al. (2005) e o estimador cópula-gráfico de Zheng e Klein (1982), que foi adaptado por Rivest e Wells (2001) para censura dependente e por Lakhal et al. (2008) para dados com estrutura de riscos semicompetitivos. O estimador cópula-gráfico é usado na aplicação do estudo de IRC.

\subsubsection{Estudo da função de sobrevivência por meio de cópulas}

O propósito desta seção é a estimação das funções de sobrevivência marginais que usualmente são de interesse para uma análise descritiva de um conjunto de dados de tempos de vida que satisfazem a estrutura de riscos semicompetitivos. Mais especificamente, será considerada a abordagem de Lakhal et al. (2008), que utiliza cópulas Arquimedianas.

Será denotado por $T_{2}$ o tempo até ocorrência do evento terminal e por $T_{1}$ o tempo até a ocorrência do evento intermediário. A variável aleatória associada ao tempo de censura será denotada por $C$. São definidas ainda as variáveis $Z=\min \left(T_{1}, T_{2}\right)$ e $\delta_{1}=I_{\left\{T_{1}<T_{2}\right\}}$, com $I_{\left\{T_{1}<T_{2}\right\}}$ uma função indicadora, ou seja, $I_{\left\{T_{1}<T_{2}\right\}}=1$ se $T_{1}<T_{2}$ e $I_{\left\{T_{1}<T_{2}\right\}}=0$, caso contrário. Observe que $\delta_{1}$ será igual a 0 se o evento terminal for observado antes do intermediário e, dessa forma, o evento intermediário não será observado. As seguintes quantidades são observáveis:

- $R=\min \left(T_{2}, C\right)$, em que $C$ é independente de $\left(T_{1}, T_{2}\right)$;

- $S=\min (Z, C)$;

- $\delta_{2}=I_{\left\{T_{2}<C\right\}}$;

- $\delta_{3}=I_{\{Z<C\}}$; 
- $\delta_{13}=\delta_{1} \delta_{3}$.

Observe que, devido à censura, $\delta_{1}$ nem sempre é conhecido. No entanto, se $\delta_{1}$ não é conhecido, $\delta_{3}=0$ e $\delta_{13}$ será igual a 0 . Com estas definições, o conjunto de dados consiste de $n$ réplicas independentes. $\Delta=\left\{\left(R_{i}, S_{i}, \delta_{2 i}, \delta_{3 i}, \delta_{13 i}\right), i=1, \cdots, n\right\}$ das variáveis observáveis $\left(R, S, \delta_{2}, \delta_{3}, \delta_{13}\right)$. Por exemplo, para o $i$-ésimo indivíduo que apresentou os dois eventos (intermediário e terminal) tem-se, $\Delta=\left\{\left(R_{i}=t_{1}, S_{i}=t_{2}, \delta_{2 i}=1, \delta_{3 i}=\right.\right.$ $\left.1, \delta_{13 i}=1\right)$. Quando no $i$-ésimo indivíduo é observado só o evento terminal, então $\Delta=\left\{\left(R_{i}=t_{2}, S_{i}=t_{2}, \delta_{2_{i}}=1, \delta_{3_{i}}=1, \delta_{13 i}=0\right)\right.$. Quando o $i$-ésimo indivíduo só apresentou o evento intermediário tem-se $\Delta=\left\{\left(R_{i}=c, S_{i}=t_{1}, \delta_{2 i}=0, \delta_{3 i}=1, \delta_{13 i}=\right.\right.$ 1) e finalmente, se nenhum dos eventos foi observado no $i$-ésimo indivíduo então $\Delta=$ $\left\{\left(R_{i}=c, S_{i}=c, \delta_{2_{i}}=0, \delta_{3 i}=0, \delta_{13 i}=0\right)\right.$.

\section{Estimador cópula-gráfico}

Este estimador foi introduzido por Zheng e Klein (1982) para estimar a função de sobrevivência sob censura dependente. Os autores assumiram uma cópula para a distribuição conjunta do tempo de falha e censura, e derivaram uma equação de estimação para as funções de sobrevivência marginais. Mais tarde, Rivest e Wells (2001) adaptaram o estimador para cópulas da família Arquimediana para dados com estrutura de riscos semicompetitivos e estudaram sua distribuição assintótica usando teoria de processos de contagem.

Segundo Lakhal et al. (2008), dentre as três propostas de estimação para $S_{T_{1}}(t)$ (não paramétrico de Fine et al. (2001), auto consistente de Jiang et al. (2005) e cópula-gráfico de Zheng e Klein (1982)), o estimador cópula-gráfico apresentou o melhor desempenho e é o apresentado a seguir. Cabe destacar que antes de obter o estimador cópula-gráfico, deve ser estimado o parâmetro da cópula $\theta$ e, para isso, Lakhal et al. (2008) usam equações de estimação. Estas equações para as cópulas Clayton, Frank e Gumbel são omitidas nesta secção, detalhes podem ser consultados em Patiño (2012).

Considere $\hat{S}_{Z}(t)=\phi_{\hat{\theta}}^{-1}\left[\phi_{\hat{\theta}}\left\{\hat{S}_{T_{1}}(t)\right\}+\phi_{\hat{\theta}}\left\{\hat{S}_{T_{2}}(t)\right\}\right]$. Se $t$ é o instante de falha observado para o evento intermediário, então

$$
\begin{aligned}
\phi_{\hat{\theta}}\left\{\hat{S}_{Z}(t)\right\} & =\phi_{\hat{\theta}}\left\{\hat{S}_{T_{1}}^{C G}(t)\right\}+\phi_{\hat{\theta}}\left\{\hat{S}_{T_{2}}(t)\right\} \mathrm{e} \\
\phi_{\hat{\theta}}\left\{\hat{S}_{Z}(t)\right\}-\phi_{\hat{\theta}}\left\{\hat{S}_{Z}\left(t^{-}\right)\right\} & =\phi_{\hat{\theta}}\left\{\hat{S}_{T_{1}}^{C G}(t)\right\}-\phi_{\hat{\theta}}\left\{\hat{S}_{T_{1}}^{C G}\left(t^{-}\right)\right\}+\phi_{\hat{\theta}}\left\{\hat{S}_{T_{2}}(t)\right\}-\phi_{\hat{\theta}}\left\{\hat{S}_{T_{2}}\left(t^{-}\right)\right\},
\end{aligned}
$$

sendo $\hat{S}_{T_{1}}^{C G}(t)$ o estimador cópula-gráfico. Como $T_{1}$ não é censurado por $T_{2}$ no tempo $t$ e como $Z=\min \left(T_{1}, T_{2}\right)$, então este instante $t$ é também um instante de falha observado para $Z$. Além disso, é um ponto de descontinuidade para $\hat{S}_{Z}($.). Por outro lado, $t$ não pode ser instante de falha para $T_{2}$ por continuidade e daí $\hat{S}_{T_{2}}($.$) não salta no instante t$.

Logo, pode-se concluir que $\phi_{\hat{\theta}}\left\{\hat{S}_{Z}(t)\right\}-\phi_{\hat{\theta}}\left\{\hat{S}_{Z}\left(t^{-}\right)\right\}=\phi_{\hat{\theta}}\left\{\hat{S}_{X}^{C G}(t)\right\}-\phi_{\hat{a}}\left\{\hat{S}_{X}^{C G}\left(t^{-}\right)\right\}$. 
Somando estes termos para todos os instantes de falha observados antes de $t$, tem-se

$$
\begin{aligned}
\sum_{S_{i} \leq t ; \delta_{13 i}=1} & \phi_{\hat{\theta}}\left\{\hat{S}_{Z}\left(S_{i}\right)\right\}-\phi_{\hat{\theta}}\left\{\hat{S}_{Z}\left(S_{i}^{-}\right)\right\}=\sum_{S_{i} \leq t ; \delta_{13 i}=1} \phi_{\hat{\theta}}\left\{\hat{S}_{T_{1}}^{C G}\left(S_{i}\right)\right\}-\phi_{\hat{\theta}}\left\{\hat{S}_{T_{1}}^{C G}\left(S_{i}^{-}\right)\right\} \\
= & \phi_{\hat{\theta}}\left\{\hat{S}_{T_{1}}^{C G}(t)\right\}-\phi_{\hat{\theta}}\left\{\hat{S}_{T_{1}}^{C G}\left(S_{k_{t}}\right)\right\}+\phi_{\hat{\theta}}\left\{\hat{S}_{T_{1}}^{C G}\left(S_{k_{t}}\right)\right\}-\phi_{\hat{\theta}}\left\{\hat{S}_{T_{1}}^{C G}\left(S_{k_{t}-1}\right)\right\} \\
& +\phi_{\hat{\theta}}\left\{\hat{S}_{T_{1}}^{C G}\left(S_{k_{t}-1}\right)\right\}-\phi_{\hat{\theta}}\left\{\hat{S}_{T_{1}}^{C G}\left(S_{k_{t}-2}\right)\right\}+\cdots+\phi_{\hat{\theta}}\left\{\hat{S}_{T_{1}}^{C G}\left(S_{2}\right)\right\}-\phi_{\hat{\theta}}\left\{\hat{S}_{T_{1}}^{C G}\left(S_{1}\right)\right\} \\
& +\phi_{\hat{\theta}}\left\{\hat{S}_{T_{1}}^{C G}\left(S_{1}\right)\right\} \\
= & \phi_{\hat{\theta}}\left\{\hat{S}_{T_{1}}^{C G}(t)\right\}
\end{aligned}
$$

em que $k_{t}$ é o número de falhas de $x$ ocorridas em $(0, \mathrm{t}]$.

Deste modo, obtém-se a seguinte expressão,

$$
\sum_{S_{i} \leq t ; \delta_{13 i}=1} \phi_{\hat{\theta}}\left\{\hat{S}_{Z}\left(S_{i}\right)\right\}-\phi_{\hat{\theta}}\left\{\hat{S}_{Z}\left(S_{i}^{-}\right)\right\}=\phi_{\hat{\theta}}\left\{\hat{S}_{T_{1}}^{C G}(t)\right\}
$$

Assim, o estimador cópula-gráfico geral é dado por

$$
\hat{S}_{T_{1}}^{C G}(t)=\phi_{\hat{\theta}}^{-1}\left[\sum_{S_{i} \leq t ; \delta_{13 i}=1} \phi_{\hat{\theta}}\left\{\hat{S}_{Z}\left(S_{i}\right)\right\}-\phi_{\hat{\theta}}\left\{\hat{S}_{Z}\left(S_{i}^{-}\right)\right\}\right] .
$$

Este estimador para as cópulas em estudo depende da função geradora inversa. Assim:

- Cópula de Clayton

$$
\hat{S}_{T_{1}}^{C G}(t)=\left(\left\{\sum_{S_{i} \leq t ; \delta_{13 i}=1}\left\{\hat{S}_{Z}\left(S_{i}\right)^{-\hat{\theta}}\right\}-\left\{\hat{S}_{Z}\left(S_{i}^{-}\right)^{-\hat{\theta}}\right\}\right\}+1\right)^{-1 / \hat{\theta}}
$$

- Cópula de Frank

$$
\hat{S}_{T_{1}}^{C G}(t)=-\frac{1}{\hat{\theta}} \ln \left[1+\left(e^{-\hat{\theta}}-1\right) \exp \left\{\sum_{S_{i} \leq t ; \delta_{13 i}=1} \ln \left(\frac{e^{-\hat{\theta}\left\{\hat{S}_{Z}\left(S_{i}\right)\right\}}-1}{e^{-\hat{\theta}\left\{\hat{S}_{Z}\left(S_{i}^{-}\right)\right\}}-1}\right)\right\}\right] .
$$

- Cópula de Gumbel,

$$
\hat{S}_{T_{1}}^{C G}(t)=\exp \left\{-\left[\sum_{S_{i} \leq t ; \delta_{13 i}=1}\left(-\ln \left(\hat{S}_{Z}\left(S_{i}\right)\right)\right)^{\hat{\alpha}}-\left(-\ln \left(\hat{S}_{Z}\left(S_{i}^{-}\right)\right)\right)^{\hat{\alpha}}\right]^{1 / \hat{\alpha}}\right\} .
$$

Depois de se obter um estimador de $S_{T_{1}}(t)$, é possível construir uma função de sobrevivência conjunta semiparamétrica (devido ao parâmetro de dependência da cópula) dos tempos até a ocorrência dos eventos intermediário e terminal. 
Na estimação da função de sobrevivência bivariada, uma alternativa pode ser o estimador paramétrico que depende do estimador das funções de sobrevivência marginais dos tempos até a ocorrência dos eventos intermediário, $T_{1}$, e terminal, $T_{2}$. O estimador semiparamétrico é baseado na função de sobrevivência bivariada dada pela cópula Arquimediana, sendo que é preciso conhecer as estimativas das funções de sobrevivência marginais dos eventos intermediário e terminal, dadas por $\hat{S}_{T_{1}}^{C G}\left(t_{1}\right)$ e $\hat{S}_{T_{2}}^{K M}\left(t_{2}\right)$, respectivamente. Assim a função de sobrevivência conjunta pode ser estimada como $\hat{S}\left(t_{1}, t_{2}\right)=\hat{C}_{\theta}\left\{\hat{S}_{T_{1}}^{C G}\left(t_{1}\right), \hat{S}_{T_{2}}^{K M}\left(t_{2}\right)\right\}$ na região $0 \leq t_{1} \leq t_{2}$. 


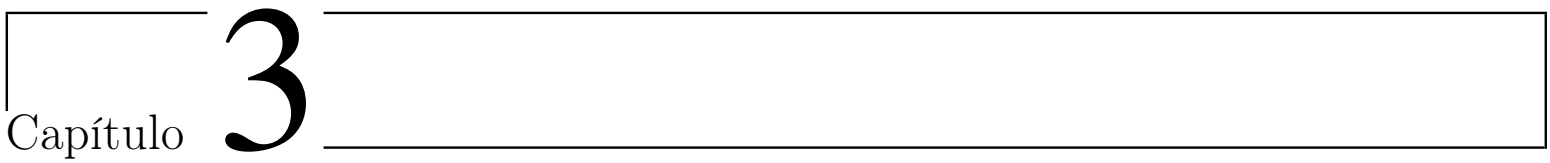

\section{Modelo de regressão bayesiano para riscos semicompetitivos}

De maneira geral, em estudos nos quais os dados apresentam uma estrutura de riscos semicompetitivos, tem-se interesse em dois tempos de falha: tempo até a progressão (ou recaída) e tempo até o óbito. Pela estrutura dos dados, observe que o tempo até a progressão nem sempre é observado. Quando observado, é necessariamente menor do que o tempo até o óbito. Em consequência, os dois tempos de interesse (tempo até o evento intermediário e tempo até o evento final) são correlacionados ou dependentes. Dessa forma, é importante que a dependência seja incorporada aos modelos propostos para tais dados. Alguns autores propõem o uso de modelo de fragilidade compartilhada (em geral, gama) para incorporar dependência, como pode ser visto em Patiño (2012), Zeng et al. (2012), Zhang et al. (2014), entre outros. Uma outra possibilidade é a utilização de cópulas para a devida incorporação da dependência entre os tempos, que é a proposta deste trabalho.

Assim como acontece em estudos de sobrevivência usuais, é muito comum que dados dessa natureza estejam sujeitos à censura. No contexto usual de sobrevivência, um dado é dito censurado se não é conhecido o instante exato do evento (ou falha), mas sabe-se que o evento (ou falha) ocorreu em um intervalo, antes ou após de um certo instante. Assim, existem vários tipos de censura, como censura intervalar à esquerda e à direita.

Na área médica, a censura à direita é o tipo mais comum por isso neste trabalho será considerada apenas esse tipo de censura. Para uma discussão em geral sobre censura, recomenda-se consultar, por exemplo, Lawless (2002), Klein e Moeschberger (2003), Colosimo e Ruiz (2006) e Hosmer et al. (2008).

Assumindo que o estudo se restringe a um horizonte finito ou de duração determinística, no caso de riscos semicompetitivos, o dado é considerado censurado se:

i) o paciente não apresentou nenhum dos eventos antes da perda de acompanhamento, que pode ocorrer num instante aleatório do horizonte do estudo ou no fim do estudo. A informação disponível é que os eventos intermediário e/ou terminal podem ter acontecido após essa perda de acompanhamento. 
ii) o paciente apresentou o evento intermediário, porém, não foi observado o evento terminal antes do fim do estudo, devido possivelmente ao fim do estudo ou a perda de acompanhamento num instante aleatório antes da ocorrência do evento terminal.

De maneira geral, em estudos em que os dados apresentam estrutura de riscos semicompetitivos e estão sujeitos a censura à direita, observa-se um conjunto de $n$ indivíduos, que são acompanhados durante um intervalo máximo de tempo $[0, \eta)$. Para cada observação na amostra, uma das quatro possibilidades a seguir pode ocorrer:

Caso 1. O indivíduo $i$ apresenta o evento terminal no instante $T_{2}$ e o evento intermediário não foi observado no intervalo $\left[0, T_{2}\right)$.

Caso 2. No intervalo de tempo $[0, \eta)$ o indivíduo $i$ apresenta o evento intermediário no instante $T_{1}$ e posteriormente o evento final no instante $T_{2}$, com $T_{1}<T_{2} \leq \eta$.

Caso 3. O indivíduo $i$ apresenta o evento intermediário no instante $T_{1}$ e até o fim do estudo não há mais informação, ou seja, é censurado no instante $\eta_{i}, T_{1}<\eta_{i}<\eta$.

Caso 4. O indivíduo $i$ não apresenta nenhum dos eventos durante o tempo de acompanhamento, ou seja, é censurado no fim do período do seu acompanhamento $\eta_{i}$.

Considerando esta estrutura, no presente capítulo são apresentados os modelos propostos, descritos com detalhes na Seção 3.1, bem como a construção da função de verossimilhança e a inferência bayesiana. Nas seções posteriores são especificadas toda a modelagem e inferência incorporando as cópulas já discutidas. Seção 3.2 cópula de Clayton, Seção 3.3 cópula de Frank e Seção 3.4 cópula de Gumbel.

\subsection{Modelagens propostas}

Conservando a estrutura de análise de Zhang et al. (2014), as modelagens propostas possuem quatro componentes. No esquema apresentado na Figura 3.1 pode-se visualizar as variáveis chaves dessas componentes. As caixas representam possíveis estados em que um indivíduo, objeto de estudo, pode ser encontrado. Ao ser incluído no estudo, o indivíduo ainda não apresentou nenhum evento e fica na situação inicial até que algum evento ocorra. Se o evento intermediário ocorre, então há transição para a caixa evento intermediário. O evento terminal seria um tipo de estado absorvente. O indivíduo pode atingir esse estado sem percorrer o evento intermediário. Note que as linhas têm um sentido e estão associadas a tempos de transições entre os estados que a linha liga. $\mathrm{Na}$ transição do estado inicial tem implícita uma variável dicotômica $E$, que indica se é observado o evento intermediário ou não, i.e., $E=1$ se o indivíduo apresenta o evento intermediário e $E=0$ caso contrário. Assim, pode ser modelada a possibilidade de ter ou não progressão, sendo esta como o evento intermediário, dadas as covariáveis $(\boldsymbol{x})$ e o tratamento $(A)$, ou seja, $\operatorname{Pr}(E=1 \mid A, \boldsymbol{x})$ forneceria a probabilidade de progressão dados $A$ e $\boldsymbol{x}$. Esta é a primeira componente das modelagens na qual é usado um modelo logito como discutido logo adiante.

Para os indivíduos na população com progressão $(E=1)$, denota-se por $T_{E}$ o tempo decorrido desde a entrada no estudo até a ocorrência do evento intermediário. Similar- 


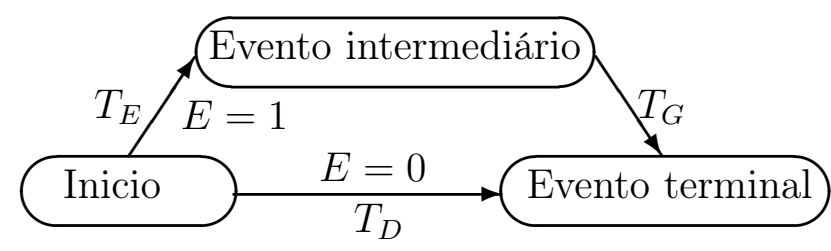

Figura 3.1: Diagrama com as variáveis chaves das modelagens propostas na estrutura de riscos semicompetitivos.

mente, denota-se por $T_{G}$ o tempo decorrido entre a ocorrência do evento intermediário e o evento terminal. Por fim, para os indivíduos que não apresentam o evento intermediário, denota-se $T_{D}$ como o tempo decorrido entre o inicio no estudo e a ocorrência do evento final ou óbito. Com os tempos assim definidos, as modelagens propostas são do tipo semi-markovianas.

Na notação a ser utilizada, $\boldsymbol{x}$ é o vetor que representa as covariáveis basais. Considerase também uma variável $A$ indicadora de tratamento ou placebo e denota-se por $\boldsymbol{z}$ o vetor de covariáveis coletadas no instante da progressão, que podem incluir um fator prognóstico para indicar mudança de tratamento. O vetor $\boldsymbol{z}$ é observado apenas para os indivíduos em que $E=1$. Ainda, inclui-se a variável $V$ binária, que indica a mudança do tratamento após a ocorrência do evento intermediário, ou seja, $V=1$ se houver mudança e $V=0$, caso contrário.

Pelo fato de os dados de IRC virem de cinco locais diferentes de tratamento, é de interesse saber se é importante ou não fazer essa diferenciação nas modelagens. Portanto, consideram-se duas situações:

1. todas as observações são independentes (modelagem fixa);

2. as observações são agrupadas e os tempos de falha de indivíduos pertencentes a um mesmo grupo podem ser dependentes (modelagem mista). Nos dados de IRC é feita a referência ao grupo como sendo a cidade que indica as localidades onde ocorreram os tratamentos (Manizales, Monteria, Rionegro, Sincelejo e Tunja).

Todos os resultados apresentados aqui foram obtidos para as duas situações. Para incorporar a possível dependência entre os tempos de falha da situação (2), considerouse um modelo misto, i.e, um modelo com efeito aleatório, acrescentando-se uma variável latente com distribuição normal.

Como a única diferença entre as situações 1 e 2 é a inclusão do efeito aleatório, optou-se neste texto por apresentar a situação mais geral, ou seja, com efeitos aleatórios e destacar as diferenças com a situação 1 quando for necessário.

Considerando os detalhes anteriormente expostos, a modelagem com efeitos aleatórios proposta apresenta as seguintes quatro componentes:

1. Modelo logito 
Modelagem da progressão da doença em função de um conjunto de covariáveis basais $\boldsymbol{x}$ e uma variável $A$ indicadora de tratamento ou placebo. Nesta situação é usado o modelo logito, dado por

$$
\operatorname{logit}(\operatorname{Pr}(E=1 \mid A, \boldsymbol{x}))=\log \left(\frac{\operatorname{Pr}(E=1 \mid A, \boldsymbol{x})}{1-\operatorname{Pr}(E=1 \mid A, \boldsymbol{x})}\right)=\alpha_{0}+A \alpha_{1}+\boldsymbol{x}^{\top} \boldsymbol{\alpha}_{\mathbf{2}},
$$

em que $\alpha_{0}, \alpha_{1}$ e $\boldsymbol{\alpha}_{\mathbf{2}}$ são coeficientes desconhecidos. Por facilidade, denota-se o preditor linear como $K_{P}=\alpha_{0}+A \alpha_{1}+\boldsymbol{x}^{\top} \boldsymbol{\alpha}_{2}$.

2. Modelo $T_{D}$

Modelagem da função de taxa de falha (ou função de risco) quando não há progressão e sim o evento terminal, óbito, dadas as covariáveis iniciais $\boldsymbol{x}$ e o tratamento $A$, dada por

$$
h_{D}\left(t_{D} \mid A, \boldsymbol{x}, E=0, b\right)=h_{0}(t) \exp \left\{A \beta_{0}+\boldsymbol{x}^{\top} \gamma_{0}+b\right\}
$$

em que

- $h_{0}(\cdot)$ é a função de risco basal desconhecida,

- $\left(\beta_{0}, \gamma_{0}\right)$ são coeficientes desconhecidos da regressão (efeitos fixos) e

- $b$ é o efeito aleatório introduzido para incorporar a dependência entre observações pertencentes a um mesmo grupo sendo $b \sim N(0, B)$. Sua função densidade é denotada por $g(b \mid B)$. No caso do modelo sem efeito aleatório, esta variável não está presente.

Para simplificar a notação, denota-se o preditor linear de efeitos fixos por $K_{D}=$ $A \beta_{0}+\boldsymbol{x}^{\top} \gamma_{0}$

De (3.2) derivam-se as expressões para as funções de sobrevivência e densidade marginal, quando a ocorrência é o óbito, dadas respectivamente por

$$
\begin{aligned}
& S_{D}\left(t_{D} \mid A, \boldsymbol{x}, E=0, b\right)=\exp \left\{-H_{0}\left(t_{D}\right) \exp \left\{K_{D}+b\right\}\right\} ; \mathrm{e} \\
& f_{D}\left(t_{D} \mid A, \boldsymbol{x}, E=0, b\right)=h_{0}\left(t_{D}\right) \exp \left\{K_{D}+b-H_{0}\left(t_{D}\right) \exp \left\{K_{D}+b\right\}\right\},
\end{aligned}
$$

sendo $H_{0}(t)=\int_{0}^{t} h_{0}(s) d s$.

3. Modelos $T_{E}$ e $T_{G}$

Modelagem da distribuição de sobrevivência bivariada quando podem ocorrer os dois eventos de interesse, progressão e óbito, dadas as covariáveis basais $\boldsymbol{x}$ o tratamento $A, E=1$ e covariáveis medidas no instante da progressão $\boldsymbol{z}$ e $V$ por meio da cópula uniparamétrica:

$$
S_{E G_{\theta}}\left(t_{E}, t_{G} \mid A, \boldsymbol{x}, E=1, \boldsymbol{z}, V, b\right)=\mathcal{C}_{\theta}\left(S_{E}\left(t_{E}\right), S_{G}\left(t_{G}\right) \mid A, \boldsymbol{x}, E=1, \boldsymbol{z}, V\right),
$$


em que $\mathcal{C}_{\theta}(\cdot, \cdot)$ é uma cópula Arquimediana ${ }^{1}$. Neste contexto, a estrutura de dependência, representada pelo parâmetro $\theta \in \Theta$, e as marginais que compõem a distribuição bivariada podem ser examinadas separadamente. A seguir serão apresentadas as funções de sobrevivência marginal $S_{E}\left(t_{E}\right)$ e $S_{G}\left(t_{G}\right)$.

Modelo $T_{E}$

Para o tempo até a progressão $T_{E}$, a função de taxa de falha associada é dada por

$$
h_{E}\left(t_{E} \mid A, \boldsymbol{x}, E=1, b\right)=h_{1}\left(t_{E}\right) \exp \left\{A \beta_{1}+\boldsymbol{x}^{\top} \boldsymbol{\gamma}_{1}+b\right\},
$$

com

- $h_{1}(\cdot)$ sendo a função de risco basal desconhecida,

- $\left(\beta_{1}, \gamma_{1}\right)$ são os coeficientes desconhecidos dos efeitos fixos da regressão, e

- $b$ é o efeito aleatório, com $b \sim N(0, B)$.

Denota-se o preditor linear de efeitos fixos por $K_{E}=A \beta_{1}+\boldsymbol{x}^{\top} \boldsymbol{\gamma}_{1}$.

As funções de sobrevivência e densidade de probabilidade associadas são, respectivamente,

$$
\begin{aligned}
& S_{E}\left(t_{E} \mid A, \boldsymbol{x}, E=1, b\right)=\exp \left\{-H_{1}\left(t_{E}\right) \exp \left\{K_{E}+b\right\}\right\}, \mathrm{e} \\
& f_{E}\left(t_{E} \mid A, \boldsymbol{x}, E=1, b\right)=h_{1}\left(t_{E}\right) \exp \left\{K_{E}+b-H_{1}\left(t_{E}\right) \exp \left\{K_{E}+b\right\}\right\},
\end{aligned}
$$

$\operatorname{com} H_{1}(t)=\int_{0}^{t} h_{1}(s) d s$.

Modelo $T_{G}$

Analogamente, para o tempo até o óbito após a progressão $T_{G}$, tem-se que

$$
h_{G}\left(t_{G} \mid A, V, \boldsymbol{z}, E=1, b\right)=h_{2}\left(t_{G}\right) \exp \left\{A \beta_{21}+V(1-A) \beta_{22}+\boldsymbol{z}^{\top} \boldsymbol{\gamma}_{2}+b\right\},
$$

em que,

- $h_{2}(\cdot)$ é a função de risco basal desconhecida,

- $\left(\boldsymbol{\beta}_{2}, \boldsymbol{\gamma}_{2}\right)$ são os coeficientes desconhecidos dos efeitos fixos da regressão e

- $b$ é o efeito aleatório, com $b \sim N(0, B)$.

Neste caso, o preditor linear é denotado por $K_{G}=A \beta_{21}+V(1-A) \beta_{22}+\boldsymbol{z}^{\top} \boldsymbol{\gamma}_{2}$. Relembremos que $V$ representa a variável indicadora de mudança de tratamento e $A$ a variável indicadora de tratamento ou placebo. Portanto, observa-se que segundo o termo $V(1-A)$ haverá mudança de tratamento quando no indivíduo foi inicialmente aplicado um placebo (e não o tratamento, i.e. $A=0$ ).

\footnotetext{
${ }^{1}$ A expressão (3.3) será apresentada com detalhes, assim como a função densidade conjunta associada a cada cópula que é usada neste trabalho, especificamente, nas seções $3.2,3.3$ e 3.4 .
} 
Com isto, tem-se que as funções de sobrevivência e densidade de probabilidade são, respectivamente,

$$
\begin{aligned}
& S_{G}\left(t_{G} \mid A, V, \boldsymbol{z}, E=1, b\right)=\exp \left\{-H_{2}\left(t_{G}\right) \exp \left\{K_{G}+b\right\}\right\} \quad \text { e } \\
& f_{G}\left(t_{G} \mid A, V, \boldsymbol{z}, E=1, b\right)=h_{2}\left(t_{G}\right) \exp \left\{K_{G}+b-H_{2}\left(t_{G}\right) \exp \left\{K_{G}+b\right\}\right\},
\end{aligned}
$$

em que $H_{2}(t)=\int_{0}^{t} h_{2}(s) d s$.

Em seguida, a partir de (3.3), obtém-se a distribuição conjunta dos dois tempos de falha ${ }^{2}$. A medida de dependência será avaliada pelo coeficiente tau de Kendall $\left(\tau_{\theta}\right)$, como descrito no Capítulo 2.

4. Distribuições basais

$\mathrm{Na}$ construção dos modelos com riscos proporcionais, o modelo exponencial por partes (piecewise exponential) é bastante usado na literatura (Ibrahim et al., 2000) (Zhang et al., 2014) para modelar a função de risco basal. Neste trabalho, o modelo exponencial por partes é escolhido para modelar cada uma das funções de risco basal envolvidas na modelagem descrita nos items anteriores, i.e., para modelar as funções de risco basais do evento terminal sem progressão $h_{0}(t)$, presente na expressão (3.2), do evento intermediário $h_{1}(t)$ da expressão (3.4) e, do evento terminal após a ocorrência do intermediário $h_{2}(t)$ da expressão (3.5). Para cada função $h_{m}(t), m=0,1,2$ considera-se uma partição finita no tempo, através dos pontos $\left\{s_{m 0}, s_{m 1}, s_{m 2}, \cdots, s_{m J_{m}}\right\}, m=0,1,2$, tal que $s_{m 0}=0<s_{m 1}<s_{m 2}<$ $\cdots<s_{m J_{m}}=\infty$ e $(0, \infty)=\bigcup_{j=1}^{J_{m}} S_{m j}, m=0,1,2$. No $j$-ésimo intervalo, assume-se uma função de risco basal constante, $h_{m}(t)=\lambda_{m j}$ para $t \in\left(s_{m, J_{m-1}}, s_{m, J_{m}}\right]$. Definindo $\boldsymbol{\lambda}_{\boldsymbol{m}}=\left(\lambda_{m 1}, \cdots, \lambda_{m J_{m}}\right)^{\top}$, a correspondente função de risco basal acumulada para $h_{m}(t)$ é dada por

$$
H_{m}\left(t \mid \boldsymbol{\lambda}_{\boldsymbol{m}}\right)=\lambda_{m j}\left(t-s_{m, j-1}\right)+\sum_{g=1}^{j-1} \lambda_{m g}\left(s_{m g}-s_{m, g-1}\right), s_{m, j-1}<t \leq s_{m j}, m=0,1,2 .
$$

Cabe ressaltar que incorporar a provável dependência entre os eventos de interesse é importante pois, segundo Hsieh et al. (2008), se a relação entre os dois eventos intermediário e terminal não for completamente especificada, então a distribuição marginal do tempo até a observação do evento intermediário é não identificável devido à possível censura dependente.

\subsubsection{Função de verossimilhança}

Uma vez definido o modelo, para fazer inferência sob o enfoque bayesiano é necessária a construção da função de verossimilhança. Para isso, é importante considerar o fato

\footnotetext{
${ }^{2} \mathrm{~A}$ distribuição conjunta também será apresentada com detalhes, para as cópulas usadas neste trabalho, nas secções $3.2,3.3$ e 3.4 .
} 
de que os dados estão sujeitos a censura à direita, conforme discutido anteriormente. Relembremos que existem duas possibilidades quando uma observação é censurada:

i) o evento intermediário é observado, porém o terminal não é e

ii) nenhum evento ocorre até o último instante de observação do paciente.

Observa-se que neste último caso não é possível saber se o paciente pertence à população que apresenta progressão $(E=1)$ ou à população que irá ao óbito sem progressão $(E=0)$.

Seja uma amostra de $N$ indivíduos. Será analisado o caso de observações agrupadas em que um efeito aleatório criará dependência dos indivíduos num mesmo grupo. Ao desconsiderar os efeitos aleatórios tem-se o caso de indivíduos independentes. Suponha $L$ grupos com $n_{l}$ observações em cada grupo, $l=1,2, \cdots, L$, tal que $N=n_{1}+n_{2}+\cdots+n_{L}$ é o total de observações.

São definidas as seguintes variáveis:

- $E_{i l}= \begin{cases}1, & \text { se o indivíduo } i \text { do grupo } l \text { apresenta progressão, } \\ 0, & \text { se o indivíduo } i \text { do grupo } l \text { não apresenta progressão. }\end{cases}$

- $Y_{E i l}$ é o tempo observado da progressão do indivíduo $i$ do grupo $l$.

- $Y_{i l}$ é o tempo observado de óbito ou censura do indivíduo $i$ do grupo $l$.

- $d_{i l}= \begin{cases}1, & \text { se a progressão foi de fato observada no indivíduo } i \text { do grupo } l \text { no } \\ & \text { tempo } Y_{E i l}, \\ 0, & \text { se a progressão não foi observada no indivíduo } i \text { do grupo } l .\end{cases}$

- $v_{i l}= \begin{cases}1, & \text { se } Y_{i l} \text { representa tempo de óbito do indivíduo } i \text { do grupo } l \\ 0, & \text { se } Y_{i l} \text { representa tempo de censura do indivíduo } i \text { do grupo } l\end{cases}$

- $A_{i l}= \begin{cases}1, & \text { se o indivíduo } i \text { do grupo } l \text { está em tratamento; } \\ 0, & \text { se o indivíduo } i \text { do grupo } l \text { é controle e recebe placebo. }\end{cases}$

- $\boldsymbol{x}_{i l}$ é o vetor das covariáveis basais do indivíduo $i$ do grupo $l$.

- $\boldsymbol{z}_{i l}$ é o vetor das covariáveis coletadas após a progressão do indivíduo $i$ do grupo $l$.

- $V_{i l}= \begin{cases}1, & \text { se houve mudança do tratamento no indivíduo } i \text { do grupo } l \text { após } \\ & \text { progressão, } \\ 0, & \text { caso contrário. }\end{cases}$

- $b_{l}$ é a variável latente, compartilhada por todas as observações do grupo $l, l=$ $1,2, \cdots, L$. Neste trabalho, assume-se que $b_{1}, b_{2}, \cdots, b_{L}$ são variáveis aleatórias i.i.d, com distribuição normal de média zero e variância $B$. 
Note que $E$ nem sempre é conhecida. De fato, se $d=1$ então $E=1$, porém se $d=0$, a observação foi censurada e então $E$ não é conhecida. Assim, os dados observados no caso do modelo com efeitos aleatórios são denotados por

$$
D_{o b s}=\left\{y_{i l}, y_{E i l}, d_{i l}, v_{i l}, A_{i l}, V_{i l}, \boldsymbol{x}_{i l}, \boldsymbol{z}_{i l}, i=1, \cdots, n_{l}, l=1, \cdots, L\right\} .
$$

No modelo sem efeitos aleatórios os dados observados são

$$
D_{o b s}=\left\{y_{i}, y_{E i}, d_{i}, v_{i}, A_{i}, V_{i}, \boldsymbol{x}_{i}, \boldsymbol{z}_{i}, i=1, \cdots, n\right\} .
$$

Em decorrência da censura, devem ser considerados os quatro casos, comentados ao inicio deste Capítulo, para a construção da verossimilhança descrita a seguir:

Caso 1. O evento intermediário da progressão da doença não é observado. Neste caso, os indivíduos apresentam só o evento terminal: óbito no instante $y_{i l}$. Se o $i$-ésimo indivíduo do l-ésimo grupo apresenta este caso, os dados observados são

$$
D_{i l}=\left(y_{i l}, d_{i l}=0, v_{i l}=1, A_{i l}, V_{i l}, \boldsymbol{x}_{i l}\right) .
$$

Neste caso, $E_{i l}$ é conhecida e igual a zero. A contribuição para a função de verossimilhança de um indivíduo neste caso incluirá as componentes (3.1) e (3.2) da modelagem, e será dada por

$$
L_{1 i l}\left(\boldsymbol{\alpha}, \beta_{0}, \gamma_{0}, \boldsymbol{\lambda}_{0}, \sigma_{b}^{2} \mid D_{i l}, b_{l}\right)=\operatorname{Pr}\left(E_{i l}=0 \mid A_{i l}, \boldsymbol{x}_{i l}\right) f_{D}\left(y_{i l} \mid A_{i l}, \boldsymbol{x}_{i l}, b_{l}\right) .
$$

Caso 2. Os indivíduos neste caso apresentam os dois eventos de interesse: progressão no instante $y_{E i l}$ e óbito no instante $y_{i l}$. Portanto, os dados observados para o $i$-ésimo indivíduo do correspondente grupo $l$, neste caso são

$$
D_{i l}=\left(y_{E i l}, y_{G i l}=y_{i l}-y_{E i l}, d_{i l}=1, v_{i l}=1, A_{i l}, V_{i l}, \boldsymbol{x}_{i l}, \boldsymbol{z}_{i l}\right) \text {. }
$$

A contribuição para a função de verossimilhança incluirá a componente (3.1) e a função densidade bivariada associada à cópula (3.3). Logo,

$$
\begin{aligned}
L_{2 i l}\left(\boldsymbol{\alpha}, \beta_{1}, \boldsymbol{\gamma}_{1}, \boldsymbol{\lambda}_{1}, \boldsymbol{\beta}_{2}, \boldsymbol{\gamma}_{2},\right. & \left.\boldsymbol{\lambda}_{2}, \theta, \sigma_{b}^{2} \mid D_{i l}, b_{l}\right)= \\
& \operatorname{Pr}\left(E_{i l}=1 \mid A_{i l}, \boldsymbol{x}_{i l}\right) f_{E G_{\theta}}\left(y_{E i l}, y_{G i l} \mid A_{i l}, V_{i l}, \boldsymbol{x}_{i l}, \boldsymbol{z}_{i l}, b_{l}\right) .
\end{aligned}
$$

Neste caso, tem-se que $d_{i l}=1, v_{i l}=1$, o que implica que $E_{i l}$ é igual a 1 . Observe que $f_{E G_{\theta}}$ depende da cópula escolhida, para Clayton será a expressão (2.4), para Frank a expressão (2.6) e para Gumbel a expressão (2.8).

Caso 3. Os indivíduos aqui apresentam só o evento intermediário da progressão da doença no instante $y_{E i l}$ e são censurados à direita no tempo $y_{i l}$. Assim, se o $i$-ésimo indivíduo do grupo $l$ estiver neste caso, terá como dados observados

$$
D_{i l}=\left(y_{E i l}, y_{G_{i} l}=y_{i l}-y_{E i l}, d_{i l}=1, v_{i l}=0, A_{i l}, V_{i l}, \boldsymbol{x}_{i l}, \boldsymbol{z}_{i l}\right) .
$$


A contribuição à função de verossimilhança correspondente contém as componentes (3.1) e a probabilidade bivarida associada à cópula (3.3) de observar o evento intermediário e sobreviver ao final é dada por

$$
\begin{aligned}
& L_{3 i l}\left(\boldsymbol{\alpha}, \beta_{1}, \boldsymbol{\gamma}_{1}, \boldsymbol{\lambda}_{1}, \boldsymbol{\beta}_{2}, \boldsymbol{\gamma}_{2}, \boldsymbol{\lambda}_{2}, \theta, \sigma_{b}^{2} \mid D_{i l}, b_{l}\right)= \\
& \operatorname{Pr}\left(E_{i l}=1 \mid A_{i l}, \boldsymbol{x}_{i l}\right) \operatorname{Pr}\left(Y_{E i l}=y_{E i l}, Y_{G i l}>y_{G i l} \mid A_{i l}, V_{i l}, \boldsymbol{x}_{i l}, \boldsymbol{z}_{i l}, b_{l}\right)= \\
& \operatorname{Pr}\left(E_{i l}=1 \mid A_{i l}, \boldsymbol{x}_{i l}\right) \int_{y_{G i l}}^{\infty} f_{E G_{\theta}}\left(y_{E i l}, u \mid A_{i l}, V_{i l}, \boldsymbol{x}_{i l}, \boldsymbol{z}_{i l}, b_{l}\right) d u
\end{aligned}
$$

em que $f_{E G_{\theta}}(u, v)$ corresponde à função densidade conjunta, indexada pelo parâmetro $\theta$ da cópula. Assim como no caso 2 , tem-se aqui que $E_{i l}=1$ é conhecido.

Caso 4. Os indivíduos neste caso não apresentam nenhum dos eventos de interesse, ou seja, o $i$-ésimo indivíduo do $l$-ésimo grupo neste caso foi censurado no instante $y_{i l}$ e não apresentou progressão antes de $y_{i l}$. Portanto, seus dados observados são

$$
D_{i l}=\left(y_{i l}, d_{i l}=0, v_{i l}=0, A_{i l}, \boldsymbol{x}_{i l}\right)
$$

e, então,

$$
\begin{aligned}
& L_{4 i l}\left(\boldsymbol{\alpha}, \beta_{0}, \boldsymbol{\gamma}_{0}, \boldsymbol{\lambda}_{0}, \beta_{1}, \boldsymbol{\gamma}_{1}, \boldsymbol{\lambda}_{1}, \theta, \sigma_{b}^{2} \mid D_{i l}, b_{l}\right)= \\
& \operatorname{Pr}\left(E_{i l}=1 \mid A_{i l}, \boldsymbol{x}_{i l}\right) S_{E_{\theta}}\left(y_{i l} \mid A_{i l}, \boldsymbol{x}_{i l}\right)+\operatorname{Pr}\left(E_{i l}=0 \mid A_{i l}, \boldsymbol{x}_{i l}\right) S_{D}\left(y_{i l} \mid A_{i l}, \boldsymbol{x}_{i l}\right)= \\
& \operatorname{Pr}\left(E_{i l}=1 \mid A_{i l}, \boldsymbol{x}_{i l}\right) \int_{Y_{G i l}}^{\infty} \int_{0}^{\infty} f_{E G_{\theta}}\left(u, v \mid A_{i l}, \boldsymbol{x}_{i l}\right) d v d u \\
& \quad+\operatorname{Pr}\left(E_{i l}=0 \mid A_{i l}, \boldsymbol{x}_{i l}\right) S_{D}\left(y_{i l} \mid A_{i l}, \boldsymbol{x}_{i l}\right) .
\end{aligned}
$$

Observe que, neste caso, o valor assumido por $E_{i l}$ não é conhecido.

Denotando por $D_{o b s}=\left(D_{i l}, i=1, \cdots, n_{l}, l=1,2, \cdots, L\right)$ o conjunto dos dados observados, com $D_{i l}$ definido para cada uma das situações anteriores, a função de verossimilhança total para o vetor de parâmetros $\boldsymbol{\xi}=\left(\boldsymbol{\alpha}^{\top}, \beta_{0}, \boldsymbol{\gamma}_{0}^{\top}, \boldsymbol{\lambda}_{0}^{\top}, \beta_{1}, \boldsymbol{\gamma}_{1}^{\top}, \boldsymbol{\lambda}_{1}^{\top}, \boldsymbol{\beta}_{2}^{\top}, \boldsymbol{\gamma}_{2}^{\top}, \boldsymbol{\lambda}_{2}^{\top}, \theta\right)^{\top}$ e $B$ é dada por

$$
\begin{aligned}
& \mathrm{L}\left(\boldsymbol{\xi}, B \mid D_{o b s}\right)=\prod_{l=1}^{L} \int_{-\infty}^{\infty} f\left(D_{o b s} \mid b_{l}\right) g\left(b_{l} \mid B\right) d b_{l} \\
& =\prod_{l=1}^{L} \int_{-\infty}^{\infty} \prod_{i=1}^{n_{l}}\left(\left[L_{1 i l}\left(\boldsymbol{\alpha}, \beta_{0}, \gamma_{0}, \boldsymbol{\lambda}_{0} \mid D_{i l}, b_{l}\right)\right]^{\mathbb{I}\left\{\begin{array}{l}
d_{i l}=0 \\
v_{i l}=1
\end{array}\right\}}\right. \\
& \times\left[L_{2 i l}\left(\boldsymbol{\alpha}, \beta_{1}, \boldsymbol{\gamma}_{1}, \boldsymbol{\lambda}_{1}, \boldsymbol{\beta}_{2}, \boldsymbol{\gamma}_{2}, \boldsymbol{\lambda}_{2}, \theta \mid D_{i l}, b_{l}\right)\right]^{\mathbb{I}\left\{\hat{v}_{i l}^{d_{i l}=1}\right\}} \\
& \left.\times\left[L_{3 i l}\left(\boldsymbol{\alpha}, \beta_{1}, \boldsymbol{\gamma}_{1}, \boldsymbol{\lambda}_{1}, \boldsymbol{\beta}_{2}, \boldsymbol{\gamma}_{2}, \boldsymbol{\lambda}_{2}, \theta \mid D_{i l}, b_{l}\right)\right]^{\mathbb{I}\left\{\sum_{i l}^{d_{i l}=1}\right\}}\right\} \\
& \left.\times\left[L_{4 i l}\left(\boldsymbol{\alpha}, \beta_{0}, \boldsymbol{\gamma}_{0}, \boldsymbol{\lambda}_{0}, \beta_{1}, \boldsymbol{\gamma}_{1}, \boldsymbol{\lambda}_{1}, \theta \mid D_{i l}, b_{l}\right)\right]^{\mathbb{I}\left\{\begin{array}{l}
d_{i l}=0 \\
v_{i l}=0
\end{array}\right.}\right) g\left(b_{l} \mid B\right) d b_{l} .
\end{aligned}
$$




\subsubsection{Inferência bayesiana}

A seguir é descrita a distribuição conjunta a posteriori, a partir da informação dos dados segundo a função de verossimilhança (3.11) e uma distribuição a priori para o vetor de parâmetros $\boldsymbol{\xi}$ e a variância do efeito aleatório $B$. Posteriormente será apresentado o algoritmo para implementar tal distribuição a posteriori de tal forma que seja possível obter estimadores para os parâmetros desconhecidos.

\section{Distribuição a posteriori}

Como indicam Lesaffre e Lawson (2012) (p. 245) e Zeng e Karim (1991), na análise de um modelo linear ou linear generalizado com efeitos aleatórios sob um enfoque bayesiano, os parâmetros $\boldsymbol{\xi}$ e $B$ são variáveis aleatórias tratadas conjuntamente com os dados observados $D_{\text {obs }}$ e os não observados $b_{l}, l=1,2, \cdots, L$, de tal forma que a distribuição a posteriori $\pi\left(\boldsymbol{\xi}, B \mid D_{\text {obs }}\right)$ é dada por

$$
\pi\left(\boldsymbol{\xi}, B \mid D_{o b s}\right)=\frac{\prod_{l=1}^{L} \int f\left(D_{o b s} \mid b_{l}\right) g\left(b_{l} \mid B\right) \pi(\boldsymbol{\xi}, B) d b_{l}}{\int \prod_{l=1}^{L} \int f\left(D_{o b s} \mid b_{l}\right) g\left(b_{l} \mid B\right) \pi(\boldsymbol{\xi}, B) d b_{l} d \boldsymbol{\xi} d B}=\frac{\mathrm{L}\left(\boldsymbol{\xi}, B \mid D_{o b s}\right) \pi(\boldsymbol{\xi}, B)}{\int \mathrm{L}\left(\boldsymbol{\xi}, B \mid D_{o b s}\right) \pi(\boldsymbol{\xi}, B) d \boldsymbol{\xi} d B} .
$$

Considerando o denominador como a constante normalizadora, independente dos parâmetros, tem-se que

$$
\pi\left(\boldsymbol{\xi}, B \mid D_{o b s}\right) \propto \mathrm{L}\left(\boldsymbol{\xi}, B \mid D_{o b s}\right) \pi(\boldsymbol{\xi}, B)
$$

em que $\mathrm{L}\left(\boldsymbol{\xi}, B \mid D_{o b s}\right)$ é a função de verossimilhança $(3.11)$ e $\pi(\boldsymbol{\xi}, B)$ é a distribuição $a$ priori conjunta para o vetor de parâmetros $\boldsymbol{\xi}$ e $B$. Assumindo independência entre $\boldsymbol{\alpha}$, $\left(\beta_{0}, \gamma_{0}\right),\left(\beta_{1}, \gamma_{1}\right),\left(\boldsymbol{\beta}_{2}, \gamma_{2}\right), \boldsymbol{\lambda}_{0}, \boldsymbol{\lambda}_{1}, \boldsymbol{\lambda}_{2}, \theta$ e $B$, a distribuição a priori conjunta pode ser expressa como o produto das distribuições a priori marginais, ou seja,

$$
\pi(\boldsymbol{\xi}, B)=\pi_{a}(\boldsymbol{\alpha}) \pi_{0}\left(\beta_{0}, \boldsymbol{\gamma}_{0}\right) \pi_{1}\left(\beta_{1}, \boldsymbol{\gamma}_{1}\right) \pi_{2}\left(\boldsymbol{\beta}_{2}, \boldsymbol{\gamma}_{2}\right) \pi_{0 \boldsymbol{\lambda}}\left(\boldsymbol{\lambda}_{0}\right) \pi_{1 \boldsymbol{\lambda}}\left(\boldsymbol{\lambda}_{1}\right) \pi_{2 \boldsymbol{\lambda}}\left(\boldsymbol{\lambda}_{2}\right) \pi(\theta) \pi(B)
$$

A distribuição a priori $\pi(\boldsymbol{\xi}, B)$ é composta por normais para os parâmetros associados aos preditores lineares, gama para os parâmetros das funções de risco basais, gama inversa para o parâmetro $\theta$ quando é usada a cópula de Clayton, normal para $\theta$ quando é usada a cópula de Frank, normal truncada no intervalo $[1, \infty)$ para $\theta$ quando tem-se a cópula de Gumbel e, finalmente, gama inversa para a variância do efeito aleatório $B$, como observa-se regularmente serem usadas na literatura. Assim, como propõem Lesaffre e Lawson (2012) e Zhang et al. (2014), $\boldsymbol{\alpha} \sim \mathrm{N}_{p_{a}}\left(\mathbf{0}, \boldsymbol{\Sigma}_{a}\right),\left(\beta_{0}, \boldsymbol{\gamma}_{0}^{\top}\right)^{\top} \sim \mathrm{N}_{p_{0}}\left(\mathbf{0}, \boldsymbol{\Sigma}_{0}\right)$, $\left(\beta_{1}, \boldsymbol{\gamma}_{1}^{\top}\right) \sim \mathrm{N}_{p_{1}}\left(\mathbf{0}, \boldsymbol{\Sigma}_{1}\right),\left(\boldsymbol{\beta}_{2}^{\top}, \boldsymbol{\gamma}_{2}^{\top}\right) \sim \mathrm{N}_{p_{2}}\left(\mathbf{0}, \boldsymbol{\Sigma}_{2}\right)$, em que $p_{a}, p_{0}, p_{1}$ e $p_{2}$ são as dimensões correspondentes aos respectivos vetores de parâmetros dos preditores lineares e as matrizes de variâncias e covariâncias $\Sigma_{a}, \Sigma_{0}, \Sigma_{1}$ e $\Sigma_{2}$ são os hiperparâmetros préespecificados. Para $\lambda_{k j}$ são assumidos $\lambda_{k j} \sim \operatorname{Gama}\left(a_{k j}, b_{k j}\right)$ com média $a_{k j} / b_{k j}$ e variância $a_{k j} / b_{k j}^{2}$ para $j=1, \cdots, J_{k}$ e $k=0,1,2$. Para $\theta$, na cópula de Clayton tem-se 
$\theta \sim \operatorname{GamaInv}\left(\kappa_{\theta}, \varsigma_{\theta}\right)$ com média $\varsigma_{\theta} /\left(\kappa_{\theta}-1\right)$ e variância $\left(\varsigma_{\theta}\right)^{2} /\left[\left(\kappa_{\theta}-1\right)^{2}\left(\kappa_{\theta}-2\right)\right]$, na cópula de Frank $\theta \sim \mathrm{N}\left(0, \sigma_{\theta}^{2}\right)$ e na cópula de Gumbel $\theta \sim \operatorname{NormalTrun}\left(0, \sigma_{\theta}^{2}, 1, \infty\right)$ com função densidade $f(\theta)=\exp \left\{-\theta^{2} / 2 \sigma_{\theta}^{2}\right\} / \sqrt{2 \pi}\left(1-\Phi\left(1 / \sigma_{\theta}\right)\right), \theta \geq 1$ e $\Phi(\cdot)$ sendo a função de distribuição normal (detalhes da distribuição normal truncada podem ser encontrados em Burkardt (2014), Johnson et al. (1994)). Para a variância do efeito aleatório $B$ é assumido $B \sim \operatorname{GamaInv}\left(\kappa_{B}, \varsigma_{B}\right)$.

Para estas distribuições a priori, são especificados os hiperparâmetros como $\boldsymbol{\Sigma}_{a}=$ $1000 \boldsymbol{I} p_{a}, \boldsymbol{\Sigma}_{0}=1000 \boldsymbol{I} p_{0}, \boldsymbol{\Sigma}_{1}=1000 \boldsymbol{I} p_{1}, \boldsymbol{\Sigma}_{2}=1000 \boldsymbol{I} p_{2}$, em que $\boldsymbol{I} p_{a}, \boldsymbol{I} p_{0}, \boldsymbol{I} p_{1}, \boldsymbol{I} p_{2}$ são as respectivas matrizes identidade. Os demais hiperparâmetros foram considerados como $a_{0 j}=b_{0 j}=0,01, a_{1 j}=0,25, b_{1 j}=0,05, a_{2 j}=b_{2 j}=0,01, \kappa_{\theta}=\varsigma_{\theta}=0,01, \sigma_{\theta}^{2}=100^{2}$ e, finalmente é escolhido adequadamente $\kappa_{B}=3$ e $\varsigma_{B}=2$. Estas configurações são conservadas tanto para o estudo de simulação quando para a aplicação.

\section{Algoritmo amostrador de Gibbs}

Após obter a distribuição a posteriori (3.12), o passo a seguir na inferência bayesiana consiste em amostrar os parâmetros desconhecidos que compõem tal distribuição. Como será mostrado posteriormente, independentemente da cópula a ser usada na modelagem, a distribuição a posteriori (3.12) não possui uma expressão reconhecida e, portanto, não é uma tarefa simples amostrar os parâmetros desconhecidos desta distribuição.

Para obter amostras dos parâmetros em (3.12) serão usados métodos de simulação Monte Carlo via cadeia de Markov (MCMC), que constituem uma classe de algoritmos de simulação sequencial. Na literatura são referenciados vários métodos para construir cadeias de Markov, sendo que os mais usados são Metrópolis-Hastings e amostrador de Gibbs.(Bonat et al., 2012)

O amostrador de Gibbs (Geman e Geman, 1984) amostra distribuições multivariadas de forma desconhecida de um vetor de parâmetros $\boldsymbol{\theta}=\left(\theta_{1}, \cdots, \theta_{k}\right)^{\top}$, a partir de amostragens sequenciais das distribuições condicionais individuais $\left[\theta_{i} \mid \theta_{-i}\right]$ que, após um conveniente período, convergem para a distribuição estacionária. A ideia é amostrar de (3.12) o vetor de parâmetros $\boldsymbol{\xi}$ e a variância do efeito aleatório $B$ a partir da distribuição marginal de cada parâmetro condicional aos demais parâmetros. Quando estas distribuições marginais não puderem ser amostradas diretamente, dado que sua densidade não é conhecida, são usadas outras estratégias de amostragem, tais como: amostragem por aceitação, amostragem por rejeção adaptativa (Gilks e Wild, 1992) e amostragem por fatias (slice sampling) (Neal, 2003), entre outras. Neste trabalho é usado o amostrador por fatias dado que não requer muitos supostos da função a ser amostrada.

Observando que $\pi\left(\boldsymbol{\xi}, B \mid D_{\text {obs }}\right)$ é uma distribuição a posteriori complexa, tanto pelo efeito aleatório quando pela censura, a introdução de variáveis latentes faz com que a distribuição a posteriori fique analiticamente tratável, como sugerido por Paquet (2008). Neste caso a ideia é usar amostragem de dados aumentados, cujos precursores foram Tanner e Wong (1987). 
A estratégia do algoritmo de dados aumentados é inserir variáveis latentes para lidar com as quantidades de variáveis desconhecidas presentes na função de verossimilhança e, assim, obter uma distribuição a posteriori aumentada da qual seja mais natural amostrar os parâmetros envolvidos.

Neste caso, para lidar com o vetor de efeitos aleatórios, utiliza-se as quantidades $\boldsymbol{b}=\left(b_{l}, l=1, \cdots, L\right)$, com $L$ sendo o número de grupos. Similarmente, para lidar com a censura é usado o vetor $\boldsymbol{E}^{*}=\left(E_{i}^{*}, i \in \mathcal{N}_{4}\right)$ em que $\mathcal{N}_{4}$ é conjunto formado pelos indivíduos observados no caso 4 . Como consequência, dados $D_{o b s}, \boldsymbol{b}$ e $\boldsymbol{E}^{*}$, a distribuição a posteriori $\pi\left(\boldsymbol{\xi}, B \mid D_{o b s}, \boldsymbol{b}, \boldsymbol{E}^{*}\right)$ é mais fácil de ser obtida do que a distribuição $a$ posteriori original $\pi\left(\boldsymbol{\xi}, B \mid D_{\text {obs }}\right)$.

Para obter a distribuição a posteriori de interesse, $\pi\left(\boldsymbol{\xi}, B \mid D_{o b s}\right)$, geram-se valores $\boldsymbol{b}$ e $\boldsymbol{E}^{*}$ através de $\pi\left(\boldsymbol{b} \mid D_{o b s}\right)$ e $\pi\left(\boldsymbol{E}^{*} \mid D_{o b s}\right)$ e então calcula-se $\pi\left(\boldsymbol{\xi}, B \mid D_{o b s}, \boldsymbol{b}, \boldsymbol{E}^{*}\right)$ com as variáveis geradas de $\boldsymbol{b}$ e $\boldsymbol{E}^{*}$. Porém, como estas distribuições para gerar $\boldsymbol{b}$ e $\boldsymbol{E}^{*}$ dependem de $\pi\left(\boldsymbol{\xi}, B \mid D_{o b s}\right)$, torna-se necessário um algoritmo iterativo e neste ponto é usado o amostrador de Gibbs. A inserção de $\boldsymbol{b}$ e $\boldsymbol{E}^{*}$ é um mecanismo para facilitar a amostragem dos parâmetros desconhecidos.

Uma vez obtida a distribuição a posteriori aumentada, obtém-se a distribuição $a$ posteriori desejada como marginal da aumentada. Supondo $\boldsymbol{b}$ independente de $\boldsymbol{E}^{*}$, temse

$$
\begin{aligned}
\pi\left(\boldsymbol{\xi}, B \mid D_{o b s}\right) & =\int_{-\infty}^{\infty} \sum_{\boldsymbol{E}^{*}} \pi\left(\boldsymbol{\xi}, B \mid D_{o b s}, \boldsymbol{b}, \boldsymbol{E}^{*}\right) \pi\left(\boldsymbol{b}, \boldsymbol{E}^{*} \mid D_{o b s}\right) d \boldsymbol{b} \\
& =\int_{-\infty}^{\infty} \sum_{\boldsymbol{E}^{*}} \pi\left(\boldsymbol{\xi}, B \mid D_{o b s}, \boldsymbol{b}, \boldsymbol{E}^{*}\right) \pi\left(\boldsymbol{b} \mid D_{o b s}\right) \pi\left(\boldsymbol{E}^{*} \mid D_{o b s}\right) d \boldsymbol{b} \\
& =\int_{-\infty}^{\infty} \sum_{\boldsymbol{E}^{*}} \pi\left(\boldsymbol{\xi}, B, \boldsymbol{b}, \boldsymbol{E}^{*} \mid D_{o b s}\right) d \boldsymbol{b} \\
& =\mathrm{L}\left(\boldsymbol{\xi}, B \mid D_{o b s}\right) \pi(\boldsymbol{\xi}, B) .
\end{aligned}
$$

Assim a distribuição a posteriori aumentada fica expressa por

$$
\pi\left(\boldsymbol{\xi}, B, \boldsymbol{b}, \boldsymbol{E}^{*} \mid D_{o b s}\right) \propto \mathrm{L}\left(\boldsymbol{\xi}, B, \boldsymbol{b}, \boldsymbol{E}^{*} \mid D_{o b s}\right) \pi(\boldsymbol{\xi}, B) .
$$

Observa-se que a expressão (3.14) corresponde à distribuição conjunta dos parâmetros e efeitos aleatórios da qual, como explicam Zeng e Karim (1991), obtêm-se as distribuições marginais que podem ser amostradas através do amostrador de Gibbs.

Detalhes da obtenção das distribuições marginais com os respectivos algoritmos de Gibbs são descritos para cada cópula considerada neste trabalho nas seções a seguir. 


\subsection{Cópula de Clayton}

Para a modelagem bayesiana descrita previamente usando a cópula de Clayton, inicialmente obtêm-se as distribuições de sobrevivência e densidade bivariadas que serão necessárias para a construção da função de verossimilhança (3.11).

Como descrito na Seção 2.2, a cópula de Clayton é dada por $\mathcal{C}_{\theta}(u, v)=\left(u^{-\theta}+v^{-\theta}-\right.$ $1)^{-\frac{1}{\theta}}$. Com $u=S_{E}\left(t_{E} \mid A, \boldsymbol{x}, E=1, b\right)$ e $v=S_{G}\left(t_{G} \mid A, \boldsymbol{x}, E=1, b\right)$, a função de sobrevivência conjunta de $T_{E}$ e $T_{G}$ para a componente do modelo (3.3) fica expressa como

$$
\begin{aligned}
S_{E G_{\theta}}\left(t_{E}, t_{G} \mid A, \boldsymbol{x}, b\right) & =\left(\left[\exp \left\{-H_{1}\left(t_{E}\right) \mathrm{e}^{K_{E}+b}\right\}\right]^{-\theta}+\left[\exp \left\{-H_{2}\left(t_{G}\right) \mathrm{e}^{K_{G}+b}\right\}\right]^{-\theta}-1\right)^{-\frac{1}{\theta}} \\
& =\left(\exp \left\{\theta H_{1}\left(t_{E}\right) \mathrm{e}^{K_{E}+b}\right\}+\exp \left\{\theta H_{2}\left(t_{G}\right) \mathrm{e}^{K_{G}+b}\right\}-1\right)^{-\frac{1}{\theta}}
\end{aligned}
$$

A função densidade bivariada fica dada por

$$
\begin{aligned}
& f_{E G_{\theta}}\left(t_{E}, t_{G} \mid A, V, \boldsymbol{x}, \boldsymbol{z}, b\right)= \\
& \frac{(1+\theta) h_{1}\left(t_{E}\right) \mathrm{e}^{K_{E}+b} h_{2}\left(t_{G}\right) \mathrm{e}^{K_{G}+b} \exp \left\{\theta H_{1}\left(t_{E}\right) \mathrm{e}^{K_{E}+b}+\theta H_{2}\left(t_{G}\right) \mathrm{e}^{K_{G}+b}\right\}}{\left[\exp \left\{\theta H_{1}\left(t_{E}\right) \mathrm{e}^{K_{E}+b}\right\}+\exp \left\{\theta H_{2}\left(t_{G}\right) \mathrm{e}^{K_{G}+b}\right\}-1\right]^{\frac{1}{\theta}+2}}
\end{aligned}
$$

Logo, a partir de (3.15) e (3.16) são obtidas as expressões da contribuição de cada caso à função de verossimilhança (3.11), segundo o indivíduo $i$ e o grupo $l$. Como indicado nas equações (3.7), (3.8), (3.9) e (3.10), tem-se, respectivamente. Os detalhes de como chegar nestas expressões podem consultados no Apêndice A.

$$
\begin{aligned}
& \text { - } L_{1 i l}\left(\boldsymbol{\alpha}, \beta_{0}, \boldsymbol{\gamma}_{0}, \boldsymbol{\lambda}_{0} \mid D_{i l}, b_{l}\right)=\frac{1}{1+\mathrm{e}^{K_{P i l}}} h_{0}\left(y_{i l} \mid \boldsymbol{\lambda}_{0}\right) \mathrm{e}^{K_{D i l}+b_{l}} \exp \left\{-H_{0}\left(y_{i l} \mid \boldsymbol{\lambda}_{0}\right) \mathrm{e}^{K_{D i l}+b_{l}}\right\}, \\
& \text { - } L_{2 i l}\left(\boldsymbol{\alpha}, \beta_{1}, \boldsymbol{\gamma}_{1}, \boldsymbol{\lambda}_{1}, \boldsymbol{\beta}_{2}, \boldsymbol{\gamma}_{2}, \boldsymbol{\lambda}_{2}, \theta \mid D_{i l}, b_{l}\right)=\frac{\mathrm{e}^{K_{P i l}}}{1+\mathrm{e}^{K_{P i l}}}(1+\theta) \\
& \times \frac{h_{1}\left(y_{E i l} \mid \boldsymbol{\lambda}_{1}\right) \mathrm{e}^{K_{E i l}+b_{l}} h_{2}\left(y_{G i l} \mid \boldsymbol{\lambda}_{2}\right) \mathrm{e}^{K_{G i}+b_{l}} \exp \left\{\theta H_{1}\left(y_{E i l} \mid \boldsymbol{\lambda}_{1}\right) \mathrm{e}^{K_{E i l}+b_{l}}+\theta H_{2}\left(y_{G i l} \mid \boldsymbol{\lambda}_{2}\right) \mathrm{e}^{K_{G i l}+b_{l}}\right\}}{\left[\exp \left\{\theta H_{1}\left(y_{E i l} \mid \boldsymbol{\lambda}_{1}\right) \mathrm{e}^{K_{E i l}+b_{l}}\right\}+\exp \left\{\theta H_{2}\left(y_{G i l} \mid \boldsymbol{\lambda}_{2}\right) \mathrm{e}^{K_{G i l}+b_{l}}\right\}-1\right]^{\frac{1}{\theta}+2}}, \\
& \text { - } L_{3 i l}\left(\boldsymbol{\alpha}, \beta_{1}, \boldsymbol{\gamma}_{1}, \boldsymbol{\lambda}_{1}, \boldsymbol{\beta}_{2}, \boldsymbol{\gamma}_{2}, \boldsymbol{\lambda}_{2}, \theta \mid D_{i l}, b_{l}\right)= \\
& \frac{\mathrm{e}^{K_{P i l}}}{1+\mathrm{e}^{K_{P i l}}} \frac{h_{1}\left(y_{E i l} \mid \boldsymbol{\lambda}_{1}\right) \mathrm{e}^{K_{E i l}+b_{l}} \exp \left\{\theta H_{1}\left(y_{E i l} \mid \boldsymbol{\lambda}_{1}\right) \mathrm{e}^{K_{E i l}+b_{l}}\right\}}{\left[\exp \left\{\theta H_{1}\left(y_{E i l} \mid \boldsymbol{\lambda}_{1}\right) \mathrm{e}^{K_{E i l}+b_{l}}\right\}+\exp \left\{\theta H_{2}\left(y_{G i l} \mid \boldsymbol{\lambda}_{2}\right) \mathrm{e}^{K_{G i}+b_{l}}\right\}-1\right]^{\frac{1}{\theta}+1}} \\
& \text { - } L_{4 i}\left(\boldsymbol{\alpha}, \beta_{0}, \gamma_{0}, \boldsymbol{\lambda}_{0}, \beta_{1}, \gamma_{1}, \boldsymbol{\lambda}_{1}, \theta \mid D_{i l}, b_{l}\right)= \\
& \frac{\mathrm{e}^{K_{P} i l}}{1+\mathrm{e}^{K_{P} i l}} \exp \left\{-H_{1}\left(y_{i l} \mid \boldsymbol{\lambda}_{1}\right) \mathrm{e}^{K_{E i l}+b_{l}}\right\}+\frac{1}{1+\mathrm{e}^{K_{P} i l}} \exp \left\{-H_{0}\left(y_{i l} \mid \boldsymbol{\lambda}_{0}\right) \mathrm{e}^{K_{D i l}+b_{l}}\right\} .
\end{aligned}
$$


Com isto, finalmente a distribuição a posteriori (3.12) para a cópula de Clayton pode ser expressa como

$$
\begin{aligned}
& \pi\left(\boldsymbol{\xi}, B \mid D_{o b s}\right) \propto\left(\prod_{l=1}^{L} \int_{-\infty}^{\infty} \prod_{i=1}^{n_{l}}\left[\frac{1}{1+\mathrm{e}^{K_{P i l}}} h_{0}\left(y_{i l} \mid \boldsymbol{\lambda}_{0}\right) \mathrm{e}^{K_{D i l}+b_{l}} \exp \left\{-H_{0}\left(y_{i l} \mid \boldsymbol{\lambda}_{0}\right) \mathrm{e}^{K_{D i l}+b_{l}}\right\}\right]^{\mathbb{I}\left\{v_{i l}^{d_{i l}=0}\right\}}\right. \\
& \times\left[\frac{\mathrm{e}^{K_{P i l}}}{1+\mathrm{e}^{K_{P i l}}}\left[\exp \left\{\theta H_{1}\left(y_{E i l} \mid \boldsymbol{\lambda}_{1}\right) \mathrm{e}^{K_{E i l}+b_{l}}\right\}+\exp \left\{\theta H_{2}\left(y_{G i l} \mid \boldsymbol{\lambda}_{2}\right) \mathrm{e}^{K_{G i l}+b_{l}}\right\}-1\right]^{-\left(\frac{1}{\theta}+2\right)}\right. \\
& \times(1+\theta) h_{1}\left(y_{E i l} \mid \boldsymbol{\lambda}_{1}\right) \mathrm{e}^{K_{E i l}+b_{l}} h_{2}\left(y_{G i l} \mid \boldsymbol{\lambda}_{2}\right) \mathrm{e}^{K_{G i}+b_{l}} \\
& \left.\times \exp \left\{\theta H_{1}\left(y_{E i l} \mid \boldsymbol{\lambda}_{1}\right) \mathrm{e}^{K_{E i l}+b_{l}}+\theta H_{2}\left(y_{G i l} \mid \boldsymbol{\lambda}_{2}\right) \mathrm{e}^{K_{G i l}+b_{l}}\right\}\right]^{\mathbb{I}\left\{\begin{array}{l}
d_{i l}=1 \\
v_{i l}=1
\end{array}\right\}} \\
& \times\left[\frac{\mathrm{e}^{K_{P i l}}}{1+\mathrm{e}^{K_{P i l}}} \frac{h_{1}\left(y_{E i l} \mid \boldsymbol{\lambda}_{1}\right) \mathrm{e}^{K_{E i l}+b_{l}} \exp \left\{\theta H_{1}\left(y_{E i l} \mid \boldsymbol{\lambda}_{1}\right) \mathrm{e}^{K_{E i l}+b_{l}}\right\}}{\left.\exp \left\{\theta H_{1}\left(y_{E i l} \mid \boldsymbol{\lambda}_{1}\right) \mathrm{e}^{K_{E i l}+b_{l}}\right\}+\exp \left\{\theta H_{2}\left(y_{G i l} \mid \boldsymbol{\lambda}_{2}\right) \mathrm{e}^{K_{G i}+b_{l}}\right\}-1\right]^{\frac{1}{\theta}+1}}\right]^{\mathbb{I}\left\{\begin{array}{c}
d_{i l}=1 \\
v_{i l}=0
\end{array}\right\}} \\
& \times\left[\frac{\mathrm{e}^{K_{P i l}}}{1+\mathrm{e}^{K_{P i l}}} \exp \left\{-H_{1}\left(y_{i l} \mid \boldsymbol{\lambda}_{1}\right) \mathrm{e}^{K_{E i l}+b_{l}}\right\}+\frac{1}{1+\mathrm{e}^{K_{P i l}}} \exp \left\{-H_{0}\left(y_{i l} \mid \boldsymbol{\lambda}_{0}\right) \mathrm{e}^{K_{D i l}+b_{l}}\right\}\right]^{\mathbb{I}\left\{\begin{array}{c}
d_{i l}=0 \\
v_{i l}=0
\end{array}\right\}} \\
& \left.\times(2 \pi B)^{-\frac{1}{2}} \exp \left\{-\frac{b_{l}^{2}}{2 B}\right\} d b_{l}\right)\left(\exp \left\{-0,5\left(\alpha_{0}, \alpha_{1}, \boldsymbol{\alpha}_{2}^{\top}\right)^{\top} \boldsymbol{\Sigma}_{\alpha}^{-1}\left(\alpha_{0}, \alpha_{1}, \boldsymbol{\alpha}_{2}^{\top}\right)\right\}\right. \\
& \times \exp \left\{-0,5\left(\beta_{0}, \boldsymbol{\gamma}_{0}^{\top}\right)^{\top} \boldsymbol{\Sigma}_{0}^{-1}\left(\beta_{0}, \boldsymbol{\gamma}_{0}^{\top}\right)\right\} \exp \left\{-0,5\left(\beta_{1}, \boldsymbol{\gamma}_{1}^{\top}\right)^{\top} \boldsymbol{\Sigma}_{1}^{-1}\left(\beta_{1}, \boldsymbol{\gamma}_{1}^{\top}\right)\right\} \\
& \times \exp \left\{-0,5\left(\boldsymbol{\beta}_{2}^{\top}, \boldsymbol{\gamma}_{2}^{\top}\right)^{\top} \boldsymbol{\Sigma}_{2}^{-1}\left(\boldsymbol{\beta}_{\mathbf{2}}^{\top}, \boldsymbol{\gamma}_{2}^{\top}\right)\right\} \prod_{j=1}^{J 0} \lambda_{0 j}^{a_{0 j}-1} \exp \left\{-b_{0 j} \lambda_{0 j}\right\} \prod_{j=1}^{J 1} \lambda_{1 j}^{a_{1 j}-1} \exp \left\{-b_{1 j} \lambda_{1 j}\right\} \\
& \left.\times \prod_{j=1}^{J 2} \lambda_{2 j}^{a_{2 j}-1} \exp \left\{-b_{2 j} \lambda_{2 j}\right\}\left(\frac{1}{\theta}\right)^{\kappa_{\theta}+1} \exp \left\{-\frac{\varsigma_{\theta}}{\theta}\right\}\left(\frac{1}{B}\right)^{\kappa_{B}+1} \exp \left\{-\frac{\varsigma_{B}}{B}\right\}\right) .
\end{aligned}
$$

Como a distribuição a priori dos parâmetros não muda, a distribuição a posteriori aumentada (3.14) fica expressa por

$$
\begin{aligned}
& \pi\left(\boldsymbol{\xi}, B, \boldsymbol{b}, \boldsymbol{E}^{*} \mid D_{o b s}\right) \propto\left(\prod_{l=1}^{L} \prod_{i=1}^{n_{l}}\left[\frac{1}{1+\mathrm{e}^{K_{P i l}}} h_{0}\left(y_{i l} \mid \boldsymbol{\lambda}_{0}\right) \mathrm{e}^{K_{D i l}+b_{l}} \exp \left\{-H_{0}\left(y_{i l} \mid \boldsymbol{\lambda}_{0}\right) \mathrm{e}^{K_{D i l}+b_{l}}\right\}\right]^{\mathbb{I}\left\{\begin{array}{l}
d_{i l l}=0 \\
v_{i l}=1
\end{array}\right\}}\right. \\
& \times\left[\frac{\mathrm{e}^{K_{P i l}}}{1+\mathrm{e}^{K_{P i l}}}\left[\exp \left\{\theta H_{1}\left(y_{E i l} \mid \boldsymbol{\lambda}_{1}\right) \mathrm{e}^{K_{E i l}+b_{l}}\right\}+\exp \left\{\theta H_{2}\left(y_{G i l} \mid \boldsymbol{\lambda}_{2}\right) \mathrm{e}^{K_{G i l}+b_{l}}\right\}-1\right]^{-\left(\frac{1}{\theta}+2\right)}(1+\theta)\right. \\
& \left.\times h_{1}\left(y_{E i l} \mid \boldsymbol{\lambda}_{1}\right) \mathrm{e}^{K_{E i l}+b_{l}} h_{2}\left(y_{G i l} \mid \boldsymbol{\lambda}_{2}\right) \mathrm{e}^{K_{G i}+b_{l}} \exp \left\{\theta H_{1}\left(y_{E i l} \mid \boldsymbol{\lambda}_{1}\right) \mathrm{e}^{K_{E i l}+b_{l}}+\theta H_{2}\left(y_{G i l} \mid \boldsymbol{\lambda}_{2}\right) \mathrm{e}^{K_{G i l}+b_{l}}\right\}\right]^{\mathbb{I}\left\{\begin{array}{c}
d_{i l}=1 \\
v_{i l}=1
\end{array}\right\}} \\
& \left.\times\left[\frac{\mathrm{e}^{K_{P i l}}}{1+\mathrm{e}^{K_{P i l}}} \frac{h_{1}\left(y_{E i l} \mid \boldsymbol{\lambda}_{1}\right) \mathrm{e}^{K_{E i l}+b_{l}} \exp \left\{\theta H_{1}\left(y_{E i l} \mid \boldsymbol{\lambda}_{1}\right) \mathrm{e}^{K_{E i l}+b_{l}}\right\}}{\left.\exp \left\{\theta H_{1}\left(y_{E i l} \mid \boldsymbol{\lambda}_{1}\right) \mathrm{e}^{K_{E i l}+b_{l}}\right\}+\exp \left\{\theta H_{2}\left(y_{G i l} \mid \boldsymbol{\lambda}_{2}\right) \mathrm{e}^{K_{G i}+b_{l}}\right\}-1\right]^{\frac{1}{\theta}+1}}\right]^{\mathbb{I}\left\{\left\{_{i l l}^{d_{i l}=1}=0\right.\right.}\right\}
\end{aligned}
$$




$$
\begin{aligned}
& \times\left[\left(\frac{\mathrm{e}^{K_{P} i l}}{1+\mathrm{e}^{K_{P} i l}} \exp \left\{-H_{1}\left(y_{i l} \mid \boldsymbol{\lambda}_{1}\right) \mathrm{e}^{K_{E i l}+b_{l}}\right\}\right)^{E_{i l}^{*}}\left(\frac{1}{1+\mathrm{e}^{K_{P} i l}} \exp \left\{-H_{0}\left(y_{i l} \mid \boldsymbol{\lambda}_{0}\right) \mathrm{e}^{K_{D i l}+b_{l}}\right\}\right)^{1-E_{i l}^{*}}\right]^{\mathbb{I}\left\{\begin{array}{c}
d_{i l}=0 \\
v_{i l}=0
\end{array}\right.} \\
& \left.\times(2 \pi B)^{-\frac{1}{2}} \exp \left\{-\frac{b_{l}^{2}}{2 B}\right\}\right) \pi(\boldsymbol{\xi}, B) .
\end{aligned}
$$

Para amostrar de (3.17), aplica-se o algoritmo amostrador de Gibbs, mais especificamente Gibbs multi-estágios (Robert e Casella, 2004) a distribuição a posteriori aumentada (3.18). Para implementar essa amostragem, primeiramente são obtidas as distribuições condicionais completas de cada parâmetro dados os parâmetros restantes, como descrito a seguir.

1. Distribuição a posteriori condicional completa de $\boldsymbol{\lambda}_{0}$

Pela independência entre alguns dos parâmetros, como pode-se observar de (3.18), tem-se que

$$
\left(\boldsymbol{\lambda}_{0} \mid \beta_{0}, \gamma_{0}, \beta_{1}, \boldsymbol{\gamma}_{1}, \boldsymbol{\lambda}_{1}, \boldsymbol{\beta}_{2}, \boldsymbol{\gamma}_{2}, \boldsymbol{\lambda}_{2}, \theta, \boldsymbol{\alpha}, B, \boldsymbol{b}, \boldsymbol{E}^{*}, D_{o b s}\right)=\left(\boldsymbol{\lambda}_{0} \mid \beta_{0}, \boldsymbol{\gamma}_{0}, \boldsymbol{b}, \boldsymbol{E}^{*}, D_{o b s}\right)
$$

Assim,

$$
\begin{aligned}
& \pi\left(\boldsymbol{\lambda}_{0} \mid \beta_{0}, \boldsymbol{\gamma}_{0}, \boldsymbol{b}, \boldsymbol{E}^{*}, D_{o b s}\right) \propto \prod_{l=1}^{L} \prod_{i=1}^{n_{l}}\left[h_{0}\left(y_{i l} \mid \boldsymbol{\lambda}_{0}\right) \exp \left\{-H_{0}\left(y_{i l} \mid \boldsymbol{\lambda}_{0}\right) \mathrm{e}^{K_{D i l}+b_{l}}\right\}\right]^{\mathbb{I}\left\{\begin{array}{l}
\left\{_{i l}=0\right. \\
v_{i l}=1
\end{array}\right\}} \\
& \quad \times\left[\exp \left\{-H_{0}\left(y_{i l} \mid \boldsymbol{\lambda}_{0}\right) \mathrm{e}^{K_{D i l}+b_{l}}\right\}\right]^{\left(1-E_{i l}^{*}\right) \mathbb{I}\left\{\begin{array}{l}
d_{i l}=0 \\
v_{i l}=0
\end{array}\right.} \prod_{j=1}^{J 0} \lambda_{0 j}^{a_{0 j}-1} \exp \left\{-b_{0 j} \lambda_{0 j}\right\} .
\end{aligned}
$$

O logaritmo da distribuição a posteriori, que será denominada função log-posteriori a menos de uma constante, é dado por

$$
\begin{gathered}
\mathcal{L}\left(\pi\left(\boldsymbol{\lambda}_{0} \mid \beta_{0}, \boldsymbol{\gamma}_{0}, \boldsymbol{b}, \boldsymbol{E}^{*}, D_{o b s}\right)\right)=\sum_{l=1}^{L} \sum_{i=1}^{n_{l}} \mathbb{I}\left\{\begin{array}{l}
d_{i l}=0 \\
v_{i l}=1
\end{array}\right\}\left(\log \left(h_{0}\left(y_{i l} \mid \boldsymbol{\lambda}_{0}\right)\right)-H_{0}\left(y_{i l} \mid \boldsymbol{\lambda}_{0}\right) \mathrm{e}^{K_{D i l}+b_{l}}\right) \\
-\sum_{l=1}^{L} \sum_{i=1}^{n_{l}}\left(1-E_{i l}^{*}\right) \mathbb{I}\left\{\begin{array}{c}
d_{i l}=0 \\
v_{i l}=0
\end{array}\right\} H_{0}\left(y_{i l} \mid \boldsymbol{\lambda}_{0}\right) \mathrm{e}^{K_{D i l}+b_{l}}+\sum_{j=1}^{J 0}\left(a_{0 j}-1\right) \ln \left(\lambda_{0 j}\right)-b_{0 j} \lambda_{0 j} .
\end{gathered}
$$

2. Distribuição a posteriori condicional completa de $\boldsymbol{\lambda}_{1}$

Pela independência dos parâmetros, neste caso tem-se que

$\left(\boldsymbol{\lambda}_{1} \mid \beta_{0}, \gamma_{0}, \boldsymbol{\lambda}_{1}, \beta_{1}, \gamma_{1}, \boldsymbol{\beta}_{2}, \gamma_{2}, \boldsymbol{\lambda}_{2}, \theta, \boldsymbol{\alpha}, B, \boldsymbol{b}, \boldsymbol{E}^{*}, D_{o b s}\right)=$ $\left(\boldsymbol{\lambda}_{1} \mid \beta_{1}, \boldsymbol{\gamma}_{1}, \boldsymbol{\beta}_{2}, \boldsymbol{\gamma}_{2}, \boldsymbol{\lambda}_{2}, \theta, \boldsymbol{b}, \boldsymbol{E}^{*}, D_{o b s}\right)$. 
Assim, de (3.18) é simples ver que

$$
\begin{aligned}
& \pi\left(\boldsymbol{\lambda}_{1} \mid \beta_{1}, \boldsymbol{\gamma}_{1}, \boldsymbol{\beta}_{2}, \boldsymbol{\gamma}_{2}, \boldsymbol{\lambda}_{2}, \theta, \boldsymbol{b}, \boldsymbol{E}^{*}, D_{o b s}\right) \propto \\
& \prod_{l=1}^{L} \prod_{i=1}^{n_{l}}\left[\frac{h_{1}\left(y_{E i l} \mid \boldsymbol{\lambda}_{1}\right) \exp \left\{\theta H_{1}\left(y_{E i l} \mid \boldsymbol{\lambda}_{1}\right) \mathrm{e}^{K_{E i l}+b_{l}}\right\}}{\left[\exp \left\{\theta H_{1}\left(y_{E i l} \mid \boldsymbol{\lambda}_{1}\right) \mathrm{e}^{K_{E i l}+b_{l}}\right\}+\exp \left\{\theta H_{2}\left(y_{G i l} \mid \boldsymbol{\lambda}_{2}\right) \mathrm{e}^{K_{G i l}+b_{l}}\right\}-1\right]^{\frac{1}{\theta}+2}}\right]^{\mathbb{I}\left\{\begin{array}{c}
d_{i l}=1 \\
v_{i l}=1
\end{array}\right\}} \\
& \times\left[\frac{h_{1}\left(y_{E i l} \mid \boldsymbol{\lambda}_{1}\right) \exp \left\{\theta H_{1}\left(y_{E i l} \mid \boldsymbol{\lambda}_{1}\right) \mathrm{e}^{K_{E i l}+b_{l}}\right\}}{\left[\exp \left\{\theta H_{1}\left(y_{E i l} \mid \boldsymbol{\lambda}_{1}\right) \mathrm{e}^{K_{E i l}+b_{l}}\right\}+\exp \left\{\theta H_{2}\left(y_{G i l} \mid \boldsymbol{\lambda}_{2}\right) \mathrm{e}^{K_{G i l}+b_{l}}\right\}-1\right]^{\frac{1}{\theta}+1}}\right]^{\mathbb{I}\left\{\begin{array}{l}
d_{i l}=1 \\
v_{i l}=0
\end{array}\right\}} \\
& \times \exp \left\{-E_{i l}^{*} \mathbb{I}\left\{\begin{array}{c}
d_{i l}=0 \\
v_{i l}=0
\end{array}\right\} H_{1}\left(y_{i l} \mid \boldsymbol{\lambda}_{1}^{*}\right) \mathrm{e}^{K_{E i l}+b_{l}}\right\} \prod_{j=1}^{J 1} \lambda_{1 j}^{a_{1 j}-1} \exp \left\{-b_{1 j} \lambda_{1 j}\right\},
\end{aligned}
$$

sendo que a função log-posteriori é dada por

$$
\begin{aligned}
\mathcal{L}(\pi & \left.\left(\boldsymbol{\lambda}_{1} \mid \beta_{1}, \boldsymbol{\gamma}_{1}, \boldsymbol{\beta}_{2}, \boldsymbol{\gamma}_{2}, \boldsymbol{\lambda}_{2}, \theta, \boldsymbol{b}, \boldsymbol{E}^{*}, D_{o b s}\right)\right)= \\
& \sum_{l=1}^{L} \sum_{i=1}^{n_{l}}\left(\mathbb{I}\left\{\begin{array}{l}
d_{i l}=1 \\
v_{i l}=1
\end{array}\right\}+\mathbb{I}\left\{\begin{array}{l}
d_{i l}=1 \\
v_{i l}=0
\end{array}\right\}\right)\left(\ln \left(h_{1}\left(y_{E i l} \mid \boldsymbol{\lambda}_{1}\right)\right)+\theta H_{1}\left(y_{E i l} \mid \boldsymbol{\lambda}_{1}\right) \mathrm{e}^{K_{E i l}+b_{l}}\right) \\
& -\sum_{l=1}^{L} \sum_{i=1}^{n_{l}}\left(\left(\frac{1}{\theta}+2\right) \mathbb{I}\left\{\begin{array}{l}
d_{i l}=1 \\
v_{i l}=1
\end{array}\right\}+\left(\frac{1}{\theta}+1\right) \mathbb{I}\left\{\begin{array}{l}
d_{i l}=1 \\
v_{i l}=0
\end{array}\right\}\right) \\
& \times \ln \left(\exp \left\{\theta H_{1}\left(y_{E i l} \mid \boldsymbol{\lambda}_{1}\right) \mathrm{e}^{K_{E i l}+b_{l}}\right\}+\exp \left\{\theta H_{2}\left(y_{G i l} \mid \boldsymbol{\lambda}_{2}\right) \mathrm{e}^{K_{G i l}+b_{l}}\right\}-1\right) \\
& -\sum_{l=1}^{L} \sum_{i=1}^{n_{l}} E_{i l}^{*} \mathbb{I}\left\{\begin{array}{l}
d_{i l}=0 \\
v_{i l}=0
\end{array}\right\} H_{1}\left(y_{i l} \mid \boldsymbol{\lambda}_{1}\right) \mathrm{e}^{K_{E i l}+b_{l}}+\sum_{j=1}^{J 1}\left(a_{1 j}-1\right) \ln \left(\lambda_{1 j}\right)-b_{1 j} \lambda_{1 j} .
\end{aligned}
$$

3. Distribuição a posteriori condicional completa de $\boldsymbol{\lambda}_{2}$

Pela independência dos parâmetros, segue que

$\left(\boldsymbol{\lambda}_{2} \mid \beta_{0}, \boldsymbol{\gamma}_{0}, \boldsymbol{\lambda}_{1}, \beta_{1}, \boldsymbol{\gamma}_{1}, \boldsymbol{\lambda}_{1}, \boldsymbol{\beta}_{2}, \boldsymbol{\gamma}_{2}, \theta, \boldsymbol{\alpha}, B, \boldsymbol{b}, \boldsymbol{E}^{*}, D_{\text {obs }}\right)=$

$$
\left(\boldsymbol{\lambda}_{2} \mid \beta_{1}, \boldsymbol{\gamma}_{1}, \boldsymbol{\lambda}_{1}, \boldsymbol{\beta}_{2}, \boldsymbol{\gamma}_{2}, \theta, \boldsymbol{b}, D_{o b s}\right) \text {. }
$$

Portanto, a partir de (3.18) a distribuição a posteriori condicional completa de $\boldsymbol{\lambda}_{2}$ é dada por

$$
\begin{aligned}
& \pi\left(\boldsymbol{\lambda}_{2} \mid \beta_{1}, \boldsymbol{\gamma}_{1}, \boldsymbol{\lambda}_{1}, \boldsymbol{\beta}_{2}, \boldsymbol{\gamma}_{2}, \theta, \boldsymbol{b}, D_{o b s}\right) \propto \\
& \prod_{l=1}^{L} \prod_{i=1}^{n_{l}}\left[\frac{h_{2}\left(y_{G i l} \mid \boldsymbol{\lambda}_{2}\right) \exp \left\{\theta H_{2}\left(y_{G i l} \mid \boldsymbol{\lambda}_{2}\right) \mathrm{e}^{K_{G i l}+b_{l}}\right\}}{\left[\exp \left\{\theta H_{1}\left(y_{E i l} \mid \boldsymbol{\lambda}_{1}\right) \mathrm{e}^{K_{E i l}+b_{l}}\right\}+\exp \left\{\theta H_{2}\left(y_{G i l} \mid \boldsymbol{\lambda}_{2}\right) \mathrm{e}^{K_{G i l}+b_{l}}\right\}-1\right]^{\frac{1}{\theta}+2}}\right]^{\mathbb{I}\left\{\begin{array}{l}
d_{i l}=1 \\
v_{i l}=1
\end{array}\right\}} \\
& \times\left[\frac{1}{\left[\exp \left\{\theta H_{1}\left(y_{E i l} \mid \boldsymbol{\lambda}_{1}\right) \mathrm{e}^{K_{E i l}}\right\}+\exp \left\{\theta H_{2}\left(y_{G i l} \mid \boldsymbol{\lambda}_{2}\right) \mathrm{e}^{K_{G i l}}\right\}-1\right]^{\frac{1}{\theta}+1}}\right]^{\mathbb{I}\left\{\begin{array}{l}
d_{i l}=1 \\
v_{i l}=0
\end{array}\right\}} \\
& \times \prod_{j=1}^{J 2} \lambda_{2 j}^{a_{2 j}-1} \exp \left\{-b_{2 j} \lambda_{2 j}\right\}
\end{aligned}
$$


e a função log-posteriori é dada por

$$
\begin{aligned}
\mathcal{L} & \left(\pi\left(\boldsymbol{\lambda}_{2} \mid \beta_{1}, \boldsymbol{\gamma}_{1}, \boldsymbol{\lambda}_{1}, \boldsymbol{\beta}_{2}, \boldsymbol{\gamma}_{2}, \theta, \boldsymbol{b}, D_{o b s}\right)\right)=\sum_{l=1}^{L} \sum_{i=1}^{n_{l}} \mathbb{I}\left\{\begin{array}{c}
d_{i l}=1 \\
v_{i l}=1
\end{array}\right\}\left(\ln \left(h_{2}\left(y_{G i l} \mid \boldsymbol{\lambda}_{2}\right)\right)\right. \\
& \left.+\theta H_{2}\left(y_{G i l} \mid \boldsymbol{\lambda}_{2}\right) \mathrm{e}^{K_{G i l}+b_{l}}\right)-\sum_{l=1}^{L} \sum_{i=1}^{n_{l}}\left(\left(\frac{1}{\theta}+2\right) \mathbb{I}\left\{\begin{array}{l}
d_{i l}=1 \\
v_{i l}=1
\end{array}\right\}+\left(\frac{1}{\theta}+1\right) \mathbb{I}\left\{\begin{array}{l}
d_{i l}=1 \\
v_{i l}=0
\end{array}\right\}\right) \\
& \times \ln \left(\exp \left\{\theta H_{1}\left(y_{E i l} \mid \boldsymbol{\lambda}_{1}\right) \mathrm{e}^{K_{E i l}+b_{l}}\right\}+\exp \left\{\theta H_{2}\left(y_{G i l} \mid \boldsymbol{\lambda}_{2}\right) \mathrm{e}^{K_{G i l}+b_{l}}\right\}-1\right) \\
& +\sum_{j=1}^{J 2}\left(a_{2 j}-1\right) \ln \left(\lambda_{2 j}\right)-b_{2 j} \lambda_{2 j} .
\end{aligned}
$$

4. Distribuição a posteriori condicional completa dos coeficientes da regressão associada ao evento terminal $\left(\beta_{0}, \gamma_{0}\right)$

Relembrando que o preditor linear dos efeitos fixos são $K_{D i l}=A_{i l} \beta_{0}+\boldsymbol{x}_{i l}^{\top} \boldsymbol{\gamma}_{0}$ e, pela independência entre parâmetros, de (3.18), pode-se observar que $\left(\beta_{0}, \boldsymbol{\gamma}_{0} \mid \boldsymbol{\lambda}_{0}, \beta_{1}, \boldsymbol{\gamma}_{1}, \boldsymbol{\lambda}_{1}, \boldsymbol{\beta}_{2}, \boldsymbol{\gamma}_{2}, \boldsymbol{\lambda}_{2}, \theta, \boldsymbol{\alpha}, B, \boldsymbol{b}, \boldsymbol{E}^{*}, D_{o b s}\right)=\left(\beta_{0}, \boldsymbol{\gamma}_{0} \mid \boldsymbol{\lambda}_{0}, \boldsymbol{b}, \boldsymbol{E}^{*}, D_{o b s}\right)$. Logo, a distribuição a posteriori condicional completa de $\left(\beta_{0}, \gamma_{0}\right)$ é dada por

$$
\begin{aligned}
& \left.\pi\left(\beta_{0}, \boldsymbol{\gamma}_{0} \mid \boldsymbol{\lambda}_{0}, \boldsymbol{b}, \boldsymbol{E}^{*}, D_{o b s}\right) \propto \prod_{l=1}^{L} \prod_{i=1}^{n_{l}}\left[\mathrm{e}^{K_{D i l}} \exp \left\{-H_{0}\left(y_{i l} \mid \boldsymbol{\lambda}_{0}\right) \mathrm{e}^{K_{D i l}+b_{l}}\right\}\right]^{\mathbb{I}\left\{\hat{v}_{i l}^{d_{i l}=0}\right\}}\right\} \\
& \quad \times\left[\exp \left\{-H_{0}\left(y_{i l} \mid \boldsymbol{\lambda}_{0}\right) \mathrm{e}^{K_{D i l}+b_{l}}\right\}\right]^{\left(1-E_{i l}^{*}\right) \mathbb{I}\left\{\left\{_{v_{i l}=0}^{d_{i l}=0}\right\}\right.} \exp \left\{-0,5\left(\beta_{0}, \boldsymbol{\gamma}_{0}^{\top}\right)^{\top} \boldsymbol{\Sigma}_{0}^{-1}\left(\beta_{0}, \boldsymbol{\gamma}_{0}^{\top}\right)\right\}
\end{aligned}
$$

A função log-posteriori de $\left(\beta_{0}, \gamma_{0}\right)$ é dada por

$$
\begin{gathered}
\mathcal{L}\left(\pi\left(\beta_{0}, \boldsymbol{\gamma}_{0} \mid \boldsymbol{\lambda}_{0}, \boldsymbol{E}^{*}, \boldsymbol{b}, D_{o b s}\right)\right)=\sum_{l=1}^{L} \sum_{i=1}^{n_{l}} \mathbb{I}\left\{\begin{array}{l}
d_{i l}=0 \\
v_{i l}=1
\end{array}\right\}\left(K_{D i l}-H_{0}\left(y_{i l} \mid \boldsymbol{\lambda}_{0}\right) \mathrm{e}^{K_{D i l}+b_{l}}\right) \\
-\sum_{l=1}^{L} \sum_{i=1}^{n_{l}}\left(1-E_{i l}^{*}\right) \mathbb{I}\left\{\begin{array}{l}
d_{i l}=0 \\
v_{i l}=0
\end{array}\right\} H_{0}\left(y_{i l} \mid \boldsymbol{\lambda}_{0}\right) \mathrm{e}^{K_{D i l}+b_{l}}-0,5\left(\beta_{0}, \boldsymbol{\gamma}_{0}^{\top}\right)^{\top} \boldsymbol{\Sigma}_{0}^{-1}\left(\beta_{0}, \boldsymbol{\gamma}_{0}^{\top}\right) .
\end{gathered}
$$

5. Distribuição a posteriori condicional completa dos coeficientes da regressão associada ao evento intermediário $\left(\beta_{1}, \gamma_{1}\right)$

Relembrando que o preditor linear de efeitos fixos é $K_{E i l}=A_{i l} \beta_{1}+\boldsymbol{x}_{i l}^{\top} \boldsymbol{\gamma}_{1}$ e pela independência entre parâmetros, tem-se que

$\left(\beta_{1}, \boldsymbol{\gamma}_{1} \mid \beta_{0}, \boldsymbol{\gamma}_{0}, \boldsymbol{\lambda}_{0}, \boldsymbol{\lambda}_{1}, \boldsymbol{\beta}_{2}, \boldsymbol{\gamma}_{2}, \boldsymbol{\lambda}_{2}, \theta, \boldsymbol{\alpha}, B, \boldsymbol{b}, \boldsymbol{E}^{*}, D_{o b s}\right)=$

$\left(\beta_{1}, \boldsymbol{\gamma}_{1} \mid \boldsymbol{\lambda}_{1}, \boldsymbol{\beta}_{2}, \boldsymbol{\gamma}_{2}, \boldsymbol{\lambda}_{2}, \theta, \boldsymbol{b}, \boldsymbol{E}^{*}, D_{o b s}\right)$. Portanto, de (3.18) pode-se observar que a 
distribuição a posteriori condicional completa de $\left(\beta_{1}, \gamma_{1}\right)$ é expressa como

$$
\begin{aligned}
& \pi\left(\beta_{1}, \boldsymbol{\gamma}_{1} \mid \boldsymbol{\lambda}_{1}, \boldsymbol{\beta}_{2}, \boldsymbol{\gamma}_{2}, \boldsymbol{\lambda}_{2}, \theta, \boldsymbol{b}, \boldsymbol{E}^{*}, D_{o b s}\right) \propto \\
& \prod_{l=1}^{L} \prod_{i=1}^{n_{l}}\left[\frac{\exp \left\{K_{E i l}+\theta H_{1}\left(y_{E i l} \mid \boldsymbol{\lambda}_{1}\right) \mathrm{e}^{K_{E i l}+b_{l}}\right\}}{\left[\exp \left\{\theta H_{1}\left(y_{E i l} \mid \boldsymbol{\lambda}_{1}\right) \mathrm{e}^{K_{E i l}+b_{l}}\right\}+\exp \left\{\theta H_{2}\left(y_{G i l} \mid \boldsymbol{\lambda}_{2}\right) \mathrm{e}^{K_{G i l}+b_{l}}\right\}-1\right]^{\frac{1}{\theta}+2}}\right]^{\mathbb{I}\left\{\begin{array}{c}
d_{i_{l}=1}=1 \\
\left.v_{i l}\right\}=1
\end{array}\right.} \\
& \times\left[\frac{\exp \left\{K_{E i l}+\theta H_{1}\left(y_{E i l} \mid \boldsymbol{\lambda}_{1}\right) \mathrm{e}^{K_{E i l}+b_{l}}\right\}}{\left[\exp \left\{\theta H_{1}\left(y_{E i l} \mid \boldsymbol{\lambda}_{1}\right) \mathrm{e}^{K_{E i l}+b_{l}}\right\}+\exp \left\{\theta H_{2}\left(y_{G i l} \mid \boldsymbol{\lambda}_{2}\right) \mathrm{e}^{K_{G i l}+b_{l}}\right\}-1\right]^{\frac{1}{\theta}+1}}\right]^{\mathbb{I}\left\{\begin{array}{c}
d_{i l}=1 \\
v_{i l}=0
\end{array}\right\}} \\
& \times \exp \left\{-E_{i l}^{*} \mathbb{I}\left\{\begin{array}{c}
d_{i l}=0 \\
v_{i l}=0
\end{array}\right\} H_{1}\left(y_{i l} \mid \boldsymbol{\lambda}_{1}\right) \mathrm{e}^{K_{E i l}+b_{l}}\right\} \exp \left\{-0,5\left(\beta_{1}, \boldsymbol{\gamma}_{1}^{\top}\right)^{\top} \boldsymbol{\Sigma}_{1}^{-1}\left(\beta_{1}, \boldsymbol{\gamma}_{1}^{\top}\right)\right\} \text {, }
\end{aligned}
$$

e a função log-posteriori é dada por

$$
\begin{aligned}
\mathcal{L}(\pi & \left.\left(\beta_{1}, \boldsymbol{\gamma}_{1} \mid \boldsymbol{\lambda}_{1}, \boldsymbol{\beta}_{2}, \boldsymbol{\gamma}_{2}, \boldsymbol{\lambda}_{2}, \theta, \boldsymbol{b}, \boldsymbol{E}^{*}, D_{o b s}, b_{l}\right)\right)= \\
& \sum_{l=1}^{L} \sum_{i=1}^{n_{l}}\left(\mathbb{I}\left\{\begin{array}{l}
d_{i l}=1 \\
v_{i l}=1
\end{array}\right\}+\mathbb{I}\left\{\begin{array}{l}
d_{i l}=1 \\
v_{i l}=0
\end{array}\right\}\right)\left(K_{E i l}+\theta H_{1}\left(y_{E i l} \mid \boldsymbol{\lambda}_{1}\right) \mathrm{e}^{K_{E i l}+b_{l}}\right) \\
& -\sum_{l=1}^{L} \sum_{i=1}^{n_{l}}\left(\left(\frac{1}{\theta}+2\right) \mathbb{I}\left\{\begin{array}{l}
d_{i l}=1 \\
v_{i l}=1
\end{array}\right\}+\left(\frac{1}{\theta}+1\right) \mathbb{I}\left\{\begin{array}{l}
d_{i l}=1 \\
v_{i l}=0
\end{array}\right\}\right) \\
& \times \ln \left(\exp \left\{\theta H_{1}\left(y_{E i l} \mid \boldsymbol{\lambda}_{1}\right) \mathrm{e}^{K_{E i l}+b_{l}}\right\}+\exp \left\{\theta H_{2}\left(y_{G i l} \mid \boldsymbol{\lambda}_{2}\right) \mathrm{e}^{K_{G i l}+b_{l}}\right\}-1\right) \\
& -\sum_{l=1}^{L} \sum_{i=1}^{n_{l}} E_{i l}^{*} \mathbb{I}\left\{\begin{array}{l}
d_{i l}=0 \\
v_{i l}=0
\end{array}\right\} H_{1}\left(y_{i l} \mid \boldsymbol{\lambda}_{1}\right) \mathrm{e}^{K_{E i l}+b_{l}}-0,5\left(\beta_{1}, \boldsymbol{\gamma}_{1}^{\top}\right)^{\top} \boldsymbol{\Sigma}_{1}^{-1}\left(\beta_{1}, \boldsymbol{\gamma}_{1}^{\top}\right) .
\end{aligned}
$$

6. Distribuição a posteriori condicional completa dos coeficientes da regressão associada ao evento terminal após a ocorrência do intermediário $\left(\boldsymbol{\beta}_{2}, \boldsymbol{\gamma}_{2}\right)$

Relembrando que o preditor linear dos efeitos fixos neste caso são $K_{G i l}=A_{i l} \beta_{21}+$ $V_{i l}\left(1-A_{i l}\right) \beta_{22}+\boldsymbol{z}_{i l}^{\top} \boldsymbol{\gamma}_{2}$ e considerando a independência entre parâmetros, tem-se que $\left(\boldsymbol{\beta}_{2}, \boldsymbol{\gamma}_{2} \mid \beta_{0}, \boldsymbol{\gamma}_{0}, \boldsymbol{\lambda}_{0}, \beta_{1}, \boldsymbol{\gamma}_{1}, \boldsymbol{\lambda}_{1}, \boldsymbol{\lambda}_{2}, \theta, \boldsymbol{\alpha}, B, \boldsymbol{b}, \boldsymbol{E}^{*}, D_{o b s}\right)=$

$\left(\boldsymbol{\beta}_{2}, \boldsymbol{\gamma}_{2} \mid \beta_{1}, \boldsymbol{\gamma}_{1}, \boldsymbol{\lambda}_{1}, \boldsymbol{\lambda}_{2}, \theta, \boldsymbol{b}, D_{\text {obs }}\right)$. De tal forma, não é difícil ver de (3.18) que a distribuição a posteriori condicional completa de $\left(\boldsymbol{\beta}_{2}, \boldsymbol{\gamma}_{2}\right)$ é

$$
\begin{aligned}
& \pi\left(\boldsymbol{\beta}_{2}, \boldsymbol{\gamma}_{2} \mid \beta_{1}, \boldsymbol{\gamma}_{1}, \boldsymbol{\lambda}_{1}, \boldsymbol{\lambda}_{2}, \theta, D_{o b s}, b_{l}\right) \propto \\
& \prod_{l=1}^{L} \prod_{i=1}^{n_{l}}\left[\frac{\exp \left\{K_{G i l}+\theta H_{2}\left(y_{G i l} \mid \boldsymbol{\lambda}_{2}\right) \mathrm{e}^{K_{G i l}+b_{l}}\right\}}{\left[\exp \left\{\theta H_{1}\left(y_{E i l} \mid \boldsymbol{\lambda}_{1}\right) \mathrm{e}^{K_{E i l}+b_{l}}\right\}+\exp \left\{\theta H_{2}\left(y_{G i l} \mid \boldsymbol{\lambda}_{2}\right) \mathrm{e}^{K_{G i l}+b_{l}}\right\}-1\right]^{\frac{1}{\theta}+2}}\right]^{\mathbb{I}\left\{\begin{array}{c}
d_{i l}=1 \\
d_{i l}=1
\end{array}\right\}} \\
& \times\left[\frac{1}{\left[\exp \left\{\theta H_{1}\left(y_{E i l} \mid \boldsymbol{\lambda}_{1}\right) \mathrm{e}^{K_{E i l}+b_{l}}\right\}+\exp \left\{\theta H_{2}\left(y_{G i l} \mid \boldsymbol{\lambda}_{2}\right) \mathrm{e}^{K_{G i}+b_{l}}\right\}-1\right]^{\frac{1}{\theta}+1}}\right]^{\mathbb{I}\left\{\begin{array}{l}
d_{i l}=1 \\
v_{i l}=0
\end{array}\right\}} \\
& \times \exp \left\{-0,5\left(\boldsymbol{\beta}_{2}^{\top}, \boldsymbol{\gamma}_{2}^{\top}\right)^{\top} \boldsymbol{\Sigma}_{2}^{-1}\left(\boldsymbol{\beta}_{2}^{\top}, \boldsymbol{\gamma}_{2}^{\top}\right)\right\} \text {, }
\end{aligned}
$$


com a função log-posteriori dada por

$$
\begin{aligned}
\mathcal{L}( & \left.\pi\left(\boldsymbol{\beta}_{2}, \boldsymbol{\gamma}_{2} \mid \beta_{1}, \boldsymbol{\gamma}_{1}, \boldsymbol{\lambda}_{1}, \boldsymbol{\lambda}_{2}, \boldsymbol{b}, \theta, D_{o b s}\right)\right)=\sum_{l=1}^{L} \sum_{i=1}^{n_{l}} \mathbb{I}\left\{\begin{array}{l}
d_{i l}=1 \\
v_{i l}=1
\end{array}\right\}\left(K_{G i l}\right. \\
& \left.+\theta H_{2}\left(y_{G i l} \mid \boldsymbol{\lambda}_{2}\right) \mathrm{e}^{K_{G i l}+b_{l}}\right)-\sum_{l=1}^{L} \sum_{i=1}^{n_{l}}\left(\left(\frac{1}{\theta}+2\right) \mathbb{I}\left\{\begin{array}{l}
d_{i l}=1 \\
i_{i l}=1
\end{array}\right\}+\left(\frac{1}{\theta}+1\right) \mathbb{I}\left\{\begin{array}{l}
d_{i l}=1 \\
i_{i l}=0
\end{array}\right\}\right) \\
& \times \ln \left(\exp \left\{\theta H_{1}\left(y_{E i l} \mid \boldsymbol{\lambda}_{1}\right) \mathrm{e}^{K_{E i l}+b_{l}}\right\}+\exp \left\{\theta H_{2}\left(y_{G i l} \mid \boldsymbol{\lambda}_{2}\right) \mathrm{e}^{K_{G i l}+b_{l}}\right\}-1\right) \\
& -0,5\left(\boldsymbol{\beta}_{2}^{\top}, \boldsymbol{\gamma}_{2}^{\top}\right)^{\top} \boldsymbol{\Sigma}_{2}^{-1}\left(\boldsymbol{\beta}_{2}^{\top}, \boldsymbol{\gamma}_{2}^{\top}\right) .
\end{aligned}
$$

7. Distribuição a posteriori condicional completa da variável latente $\boldsymbol{E}^{*}$

Para o vetor de variáveis latentes $\boldsymbol{E}^{*}$, de (3.18) pode-se observar que

$\left(\boldsymbol{E}^{*} \mid \beta_{0}, \boldsymbol{\gamma}_{0}, \boldsymbol{\lambda}_{0}, \beta_{1}, \boldsymbol{\gamma}_{1}, \boldsymbol{\lambda}_{1}, \boldsymbol{\beta}_{2}, \boldsymbol{\gamma}_{2}, \boldsymbol{\lambda}_{2}, \theta, \boldsymbol{\alpha}, B, \boldsymbol{b}, D_{\text {obs }}\right)=$

$\left(\boldsymbol{E}^{*} \mid \boldsymbol{\alpha}, \beta_{0}, \boldsymbol{\gamma}_{0}, \boldsymbol{\lambda}_{0}, \beta_{1}, \boldsymbol{\gamma}_{1}, \boldsymbol{\lambda}_{1}, \theta, \boldsymbol{b}, D_{\text {obs }}\right)$. De tal forma vemos que a expressão da distribuição a posteriori condicional completa para $\boldsymbol{E}^{*}$ pode ser expressa como

$$
\begin{aligned}
& \pi\left(\boldsymbol{E}^{*} \mid \boldsymbol{\alpha}, \beta_{0}, \gamma_{0}, \boldsymbol{\lambda}_{0}, \beta_{1}, \gamma_{1}, \boldsymbol{\lambda}_{1}, \theta, \boldsymbol{b}, D_{o b s}\right) \propto \prod_{l=1}^{L} \prod_{i=1}^{n_{l}} \\
& \left(\left[\frac{\exp \left\{K_{P i l}-H_{1}\left(y_{i l} \mid \boldsymbol{\lambda}_{1}\right) \mathrm{e}^{K_{E i l}+b_{l}}\right\}}{1+\mathrm{e}^{K_{P i l}}}\right]^{E_{i l}^{*}}\left[\frac{\exp \left\{-H_{0}\left(y_{i l} \mid \boldsymbol{\lambda}_{0}\right) \mathrm{e}^{K_{D i l}+b_{l}}\right\}}{1+\mathrm{e}^{K_{P i l}}}\right]^{1-E_{i l}^{*}}\right)^{\mathbb{I}\left\{\begin{array}{c}
\left.d_{i l}^{d_{i l}=0}\right\} \\
\left.v_{i l}\right\}
\end{array}\right.} .
\end{aligned}
$$

8. Distribuição a posteriori condicional completa do parâmetro da cópula $\theta$ Novamente, considerando a independência entre parâmetros, tem-se que $\left(\theta \mid \beta_{0}, \boldsymbol{\gamma}_{0}, \boldsymbol{\lambda}_{0}, \beta_{1}, \boldsymbol{\gamma}_{1}, \boldsymbol{\lambda}_{1}, \boldsymbol{\beta}_{2}, \boldsymbol{\gamma}_{2}, \boldsymbol{\lambda}_{2}, \boldsymbol{\alpha}, B, \boldsymbol{b}, \boldsymbol{E}^{*}, D_{o b s}\right)=$ $\left(\theta \mid \beta_{1}, \boldsymbol{\gamma}_{1}, \boldsymbol{\lambda}_{1}, \boldsymbol{\beta}_{2}, \boldsymbol{\gamma}_{2}, \boldsymbol{\lambda}_{2}, \boldsymbol{b}, \boldsymbol{E}^{*}, D_{\text {obs }}\right)$.

Assim, podemos ver de (3.18) que a distribuição a posteriori condicional completa para $\theta$ fica expressa como

$$
\begin{aligned}
& \pi\left(\theta \mid \beta_{1}, \boldsymbol{\gamma}_{1}, \boldsymbol{\lambda}_{1}, \boldsymbol{\beta}_{2}, \boldsymbol{\gamma}_{2}, \boldsymbol{\lambda}_{2}, \boldsymbol{b}, \boldsymbol{E}^{*}, D_{o b s}\right) \propto \\
& \prod_{l=1}^{L} \prod_{i=1}^{n_{l}}\left[\frac{(1+\theta) \exp \left\{\theta H_{1}\left(y_{E i l} \mid \boldsymbol{\lambda}_{1}\right) \mathrm{e}^{K_{E i l}+b_{l}}+\theta H_{2}\left(y_{G i l} \mid \boldsymbol{\lambda}_{2}\right) \mathrm{e}^{K_{G i l}+b_{l}}\right\}}{\left[\exp \left\{\theta H_{1}\left(y_{E i l} \mid \boldsymbol{\lambda}_{1}\right) \mathrm{e}^{K_{E i l}+b_{l}}\right\}+\exp \left\{\theta H_{2}\left(y_{G i l} \mid \boldsymbol{\lambda}_{2}\right) \mathrm{e}^{K_{G i l}+b_{l}}\right\}-1\right]^{\frac{1}{\theta}+2}}\right]^{\mathbb{I}\left\{\begin{array}{l}
d_{i l}=1 \\
v_{i l}=1
\end{array}\right\}} \\
& \times\left[\frac{\exp \left\{\theta H_{1}\left(y_{E i l} \mid \boldsymbol{\lambda}_{1}\right) \mathrm{e}^{K_{E i}+b_{l}}\right\}}{\left[\exp \left\{\theta H_{1}\left(y_{E i l} \mid \boldsymbol{\lambda}_{1}\right) \mathrm{e}^{K_{E i l}+b_{l}}\right\}+\exp \left\{\theta H_{2}\left(y_{G i l} \mid \boldsymbol{\lambda}_{2}\right) \mathrm{e}^{K_{G i l}+b_{l}}\right\}-1\right]^{\frac{1}{\theta}+1}}\right]^{\mathbb{I}\left\{\begin{array}{l}
d_{i l}=1 \\
v_{i l}=0
\end{array}\right\}} \\
& \times\left(\frac{1}{\theta}\right)^{\kappa_{\theta}+1} \exp \left\{-\frac{\varsigma_{\theta}}{\theta}\right\} \text {, }
\end{aligned}
$$


e a função log-posteriori por

$$
\begin{aligned}
& \mathcal{L}\left(\pi\left(\theta \mid \beta_{1}, \boldsymbol{\gamma}_{1}, \boldsymbol{\lambda}_{1}, \boldsymbol{\beta}_{2}, \boldsymbol{\gamma}_{2}, \boldsymbol{\lambda}_{2}, \boldsymbol{b}, \boldsymbol{E}^{*}, D_{o b s}\right)\right)= \\
& \quad \sum_{l=1}^{L} \sum_{i=1}^{n_{l}} \mathbb{I}\left\{\begin{array}{l}
d_{i l}=1 \\
v_{i l}=1
\end{array}\right\}\left(\ln (1+\theta)+\theta\left(H_{1}\left(y_{E i l} \mid \boldsymbol{\lambda}_{1}\right) \mathrm{e}^{K_{E i l}+b_{l}}+H_{2}\left(y_{G i l} \mid \boldsymbol{\lambda}_{2}\right) \mathrm{e}^{K_{G i l}+b_{l}}\right)\right) \\
& \quad+\sum_{l=1}^{L} \sum_{i=1}^{n_{l}} \mathbb{I}\left\{\begin{array}{l}
d_{i l}=1 \\
v_{i l}=0
\end{array}\right\} \theta H_{1}\left(y_{E i l} \mid \boldsymbol{\lambda}_{1}\right) \mathrm{e}^{K_{E i l}+b_{l}}-\sum_{l=1}^{L} \sum_{i=1}^{n_{l}}\left(\left(\frac{1}{\theta}+2\right) \mathbb{I}\left\{\begin{array}{l}
d_{i l}=1 \\
v_{i l}=1
\end{array}\right\}+\left(\frac{1}{\theta}+1\right)\right. \\
& \left.\quad \times \mathbb{I}\left\{\begin{array}{l}
d_{i l}=1 \\
v_{i l}=0
\end{array}\right\}\right) \ln \left(\exp \left\{\theta H_{1}\left(y_{E i l} \mid \boldsymbol{\lambda}_{1}\right) \mathrm{e}^{K_{E i l}+b_{l}}\right\}+\exp \left\{\theta H_{2}\left(y_{G i l} \mid \boldsymbol{\lambda}_{2}\right) \mathrm{e}^{K_{G i l}+b_{l}}\right\}-1\right) \\
& \quad-\left(\kappa_{\theta}+1\right) \log (\theta)-\frac{\varsigma_{\theta}}{\theta} .
\end{aligned}
$$

9. Distribuição a posteriori condicional completa dos coeficientes do modelo logito $\alpha$

Pela independência dos parâmetros, pode-se observar que de (3.18),

$$
\left(\boldsymbol{\alpha} \mid \beta_{0}, \boldsymbol{\gamma}_{0}, \boldsymbol{\lambda}_{0}, \beta_{1}, \boldsymbol{\gamma}_{1}, \boldsymbol{\lambda}_{1}, \boldsymbol{\beta}_{2}, \boldsymbol{\gamma}_{2}, \boldsymbol{\lambda}_{2}, \theta, B, \boldsymbol{b}, \boldsymbol{E}^{*}, D_{o b s}\right)=\left(\boldsymbol{\alpha} \mid \boldsymbol{E}^{*}, D_{o b s}\right) .
$$

Finalmente, relembrando que $K_{P i l}=\alpha_{0}+A_{i l} \alpha_{1}+\boldsymbol{x}_{i l}^{\top} \boldsymbol{\alpha}_{2}$, tem-se a distribuição $a$ posteriori condicional completa de $\boldsymbol{\alpha}$ dada por

$$
\begin{aligned}
& \pi\left(\boldsymbol{\alpha} \mid \boldsymbol{E}^{*}, D_{o b s}\right) \propto \\
& \prod_{l=1}^{L} \prod_{i=1}^{n_{l}}\left[\frac{\mathrm{e}^{K_{\text {Pil }}}}{1+\mathrm{e}^{K_{\text {Pil }}}}\right]^{\mathbb{I}\left\{\begin{array}{c}
d_{i l}=1 \\
v_{i l}=1
\end{array}\right\}+\mathbb{I}\left\{\begin{array}{l}
d_{i l}=1 \\
v_{i l}=0
\end{array}\right\}+E_{i}^{*} \mathbb{I}\left\{\begin{array}{l}
d_{i=0}=0 \\
v_{i}=0
\end{array}\right\}}\left[\frac{1}{1+\mathrm{e}^{K_{P} i l}}\right]^{\mathbb{I}\left\{\begin{array}{l}
d_{i l}=0 \\
v_{i l}=1
\end{array}\right\}+\left(1-E_{i l}^{*}\right) \mathbb{I}\left\{\begin{array}{l}
d_{i l}=0 \\
v_{i l}=0
\end{array}\right\}} \\
& \times \exp \left\{-0,5\left(\alpha_{0}, \alpha_{1}, \boldsymbol{\alpha}_{2}^{\top}\right)^{\top} \boldsymbol{\Sigma}_{\alpha}^{-1}\left(\alpha_{0}, \alpha_{1}, \boldsymbol{\alpha}_{2}^{\top}\right)\right\} .
\end{aligned}
$$

com a função log-posteriori dada por

$$
\begin{aligned}
& \mathcal{L}\left(\pi\left(\boldsymbol{\alpha} \mid \boldsymbol{E}^{*}, D_{o b s}\right)\right)=-\sum_{l=1}^{L} \sum_{i=1}^{n_{l}}\left(\mathbb{I}\left\{\begin{array}{l}
d_{i l}=1 \\
v_{i l}=1
\end{array}\right\}+\mathbb{I}\left\{\begin{array}{l}
d_{i l}=1 \\
v_{i l}=0
\end{array}\right\}+E_{i l}^{*} \mathbb{I}\left\{\begin{array}{l}
d_{i l}=0 \\
v_{i l}=0
\end{array}\right\}\right) \\
& \quad \times \ln \left(\exp \left\{-K_{P i l}\right\}+1\right)-\sum_{l=1}^{L} \sum_{i=1}^{n_{l}}\left(\mathbb{I}\left\{\begin{array}{l}
d_{i l}=0 \\
v_{i l}=1
\end{array}\right\}+\left(1-E_{i l}^{*}\right) \mathbb{I}\left\{\begin{array}{l}
d_{i l}=0 \\
v_{i l}=0
\end{array}\right\}\right) \ln \left(\exp \left\{K_{P i l}\right\}+1\right) \\
& \quad-0,5\left(\alpha_{0}, \alpha_{1}, \boldsymbol{\alpha}_{2}^{\top}\right)^{\top} \boldsymbol{\Sigma}_{\alpha}^{-1}\left(\alpha_{0}, \alpha_{1}, \boldsymbol{\alpha}_{2}^{\top}\right) .
\end{aligned}
$$

10. Distribuição a posteriori condicional completa da variância do efeito aleatório $B$ Observe que de (3.18), a variância $B$ só depende do vetor de efeitos aleatórios $\boldsymbol{b}$, ou seja,

$$
\left(B \mid \beta_{0}, \boldsymbol{\gamma}_{0}, \boldsymbol{\lambda}_{0}, \beta_{1}, \boldsymbol{\gamma}_{1}, \boldsymbol{\lambda}_{1}, \boldsymbol{\beta}_{2}, \boldsymbol{\gamma}_{2}, \boldsymbol{\lambda}_{2}, \theta, \boldsymbol{\alpha}, \boldsymbol{b}, \boldsymbol{E}^{*}, D_{o b s}\right)=(B \mid \boldsymbol{b}) .
$$

Assumindo que os efeitos aleatórios $\boldsymbol{b}=\left(b_{1}, \cdots, b_{L}\right)^{\top}$ são i.i.d $\operatorname{Normal}(0, B)$, tem- 
se

$$
\begin{aligned}
\pi(B \mid \boldsymbol{b}) & \propto \prod_{l=1}^{L}\left[(2 \pi B)^{-\frac{1}{2}} \exp \left\{-\frac{b_{l}^{2}}{2 B}\right\}\right]\left(\frac{1}{B}\right)^{\kappa_{B}+1} \exp \left\{-\frac{\varsigma_{B}}{B}\right\} \\
& =(2 \pi B)^{-\frac{L}{2}} \exp \left\{-\frac{\sum_{l=1}^{L} b_{l}^{2}}{2 B}\right\}\left(\frac{1}{B}\right)^{\kappa_{B}+1} \exp \left\{-\frac{\varsigma_{B}}{B}\right\} \\
& =\left(\frac{1}{B}\right)^{\frac{L}{2}+\kappa_{B}+1} \exp \left\{-\frac{1}{B}\left(\frac{\sum_{l=1}^{L} b_{l}^{2}}{2}+\varsigma_{B}\right)\right\} .
\end{aligned}
$$

Portanto, $(B \mid \boldsymbol{b}) \sim \operatorname{Gama} \operatorname{Inversa}\left(\frac{L}{2}+\kappa_{B} ; \frac{\sum_{l=1}^{L} b_{l}^{2}}{2}+\varsigma_{B}\right)$.

11. Distribuição a posteriori condicional completa do vetor de efeitos aleatórios $\boldsymbol{b}$ Examinando a distribuição a posteriori aumentada (3.18), verifica-se que $\left(\boldsymbol{b} \mid \beta_{0}, \boldsymbol{\gamma}_{0}, \boldsymbol{\lambda}_{0}, \beta_{1}, \boldsymbol{\gamma}_{1}, \boldsymbol{\lambda}_{1}, \boldsymbol{\beta}_{2}, \boldsymbol{\gamma}_{2}, \boldsymbol{\lambda}_{2}, \theta, \boldsymbol{\alpha}, B, \boldsymbol{E}^{*}, D_{o b s}\right)=$ $\left(\boldsymbol{b} \mid \beta_{0}, \boldsymbol{\gamma}_{0}, \beta_{1}, \boldsymbol{\gamma}_{1}, \boldsymbol{\beta}_{2}, \boldsymbol{\gamma}_{2}, \boldsymbol{\lambda}_{0}, \boldsymbol{\lambda}_{1}, \boldsymbol{\lambda}_{2}, \theta, B, D_{\text {obs }}\right)$.

Finalmente, a distribuição a posteriori condicional completa dos efeitos aleatórios é dada por

$$
\begin{aligned}
& \pi\left(\boldsymbol{b} \mid \beta_{0}, \boldsymbol{\gamma}_{0}, \beta_{1}, \boldsymbol{\gamma}_{1}, \boldsymbol{\beta}_{2}, \boldsymbol{\gamma}_{2}, \boldsymbol{\lambda}_{0}, \boldsymbol{\lambda}_{1}, \boldsymbol{\lambda}_{2}, \theta, B, D_{o b s}\right) \propto \\
& \prod_{l=1}^{L} \prod_{i=1}^{n_{l}}\left[\exp \left\{b_{l}-H_{0}\left(y_{i l} \mid \boldsymbol{\lambda}_{0}\right) \mathrm{e}^{K_{D i l}+b_{l}}\right\}\right]^{\mathbb{I}\left\{\begin{array}{l}
d_{i l}=0 \\
v_{i l}=1
\end{array}\right\}} \\
& \times\left[\frac{\exp \left\{2 b_{l}+\theta H_{1}\left(y_{E i l} \mid \boldsymbol{\lambda}_{1}\right) \mathrm{e}^{K_{E i l}+b_{l}}+\theta H_{2}\left(y_{G i l} \mid \boldsymbol{\lambda}_{2}\right) \mathrm{e}^{K_{G i l}+b_{l}}\right\}}{\left[\exp \left\{\theta H_{1}\left(y_{E i l} \mid \boldsymbol{\lambda}_{1}\right) \mathrm{e}^{K_{E i l}+b_{l}}\right\}+\exp \left\{\theta H_{2}\left(y_{G i l} \mid \boldsymbol{\lambda}_{2}\right) \mathrm{e}^{K_{G i l}+b_{l}}\right\}-1\right]^{\frac{1}{\theta}+2}}\right]^{\mathbb{I}\left\{\begin{array}{l}
d_{i l}=1 \\
v_{i l}=1
\end{array}\right\}} \\
& \times\left[\frac{\exp \left\{b_{l}+\theta H_{1}\left(y_{E i l} \mid \boldsymbol{\lambda}_{1}\right) \mathrm{e}^{K_{E i l}+b_{l}}\right\}}{\left[\exp \left\{\theta H_{1}\left(y_{E i l} \mid \boldsymbol{\lambda}_{1}\right) \mathrm{e}^{K_{E i l}+b_{l}}\right\}+\exp \left\{\theta H_{2}\left(y_{G i l} \mid \boldsymbol{\lambda}_{2}\right) \mathrm{e}^{K_{G i l}+b_{l}}\right\}-1\right]^{\frac{1}{\theta}+1}}\right]^{\mathbb{I}\left\{\begin{array}{c}
d_{i l}=1 \\
v_{i l}=0
\end{array}\right\}} \\
& \times\left[\exp \left\{-E_{i l}^{*} H_{1}\left(y_{i l} \mid \boldsymbol{\lambda}_{1}\right) \mathrm{e}^{K_{E i l}+b_{l}}\right\} \exp \left\{-\left(1-E_{i l}^{*}\right) H_{0}\left(y_{i l} \mid \boldsymbol{\lambda}_{0}\right) \mathrm{e}^{K_{D i l}+b_{l}}\right\}\right]^{\mathbb{I}\left\{\begin{array}{l}
d_{i l}=0 \\
v_{i l}=0
\end{array}\right\}} \\
& \times \exp \left\{-\frac{b_{l}^{2}}{2 B}\right\} \text {, }
\end{aligned}
$$


com a função log-posteriori expressa como

$$
\begin{aligned}
& \mathcal{L}\left(\pi\left(\boldsymbol{b} \mid \beta_{0}, \boldsymbol{\gamma}_{0}, \beta_{1}, \boldsymbol{\gamma}_{1}, \boldsymbol{\beta}_{2}, \boldsymbol{\gamma}_{2}, \boldsymbol{\lambda}_{0}, \boldsymbol{\lambda}_{1}, \boldsymbol{\lambda}_{2}, \theta, B, D_{o b s}\right)\right)= \\
& \quad \sum_{l=1}^{L} \sum_{i=1}^{n_{l}} \mathbb{I}\left\{\begin{array}{l}
d_{i l}=0 \\
v_{i l}=1
\end{array}\right\}\left(b_{l}-H_{0}\left(y_{i l} \mid \boldsymbol{\lambda}_{0}\right) \mathrm{e}^{K_{D i l}+b_{l}}\right)+\sum_{l=1}^{L} \sum_{i=1}^{n_{l}} \mathbb{I}\left\{\begin{array}{l}
d_{i l}=1 \\
v_{i l}=1
\end{array}\right\} \\
& \quad \times\left(2 b_{l}+\theta H_{1}\left(y_{E i l} \mid \boldsymbol{\lambda}_{1}\right) \mathrm{e}^{K_{E i l}+b_{l}}+\theta H_{2}\left(y_{G i l} \mid \boldsymbol{\lambda}_{2}\right) \mathrm{e}^{K_{G i l}+b_{l}}\right)+\sum_{l=1}^{L} \sum_{i=1}^{n_{l}} \mathbb{I}\left\{\begin{array}{l}
d_{i l}=1 \\
v_{i l}=0
\end{array}\right\} \\
& \quad \times\left(b_{l}+\theta H_{1}\left(y_{E i l} \mid \boldsymbol{\lambda}_{1}\right) \mathrm{e}^{K_{E i l}+b_{l}}\right)-\sum_{l=1}^{L} \sum_{i=1}^{n_{l}}\left(\left(\frac{1}{\theta}+2\right) \mathbb{I}\left\{\begin{array}{l}
d_{i l}=1 \\
v_{i l}=1
\end{array}\right\}+\left(\frac{1}{\theta}+1\right) \mathbb{I}\left\{\begin{array}{l}
d_{i l}=1 \\
v_{i l}=0
\end{array}\right\}\right) \\
& \quad \times \ln \left(\exp \left\{\theta H_{1}\left(y_{E i l} \mid \boldsymbol{\lambda}_{1}\right) \mathrm{e}^{K_{E i l}+b_{l}}\right\}+\exp \left\{\theta H_{2}\left(y_{G i l} \mid \boldsymbol{\lambda}_{2}\right) \mathrm{e}^{K_{G i l}+b_{l}}\right\}-1\right) \\
& \quad-\sum_{l=1}^{L} \sum_{i=1}^{n_{l}} \mathbb{I}\left\{\begin{array}{l}
d_{i l}=0 \\
v_{i l}=0
\end{array}\right\}\left(E_{i l}^{*} H_{1}\left(y_{i l} \mid \boldsymbol{\lambda}_{1}\right) \mathrm{e}^{K_{E i l}+b_{l}}+\left(1-E_{i l}^{*}\right) H_{0}\left(y_{i l} \mid \boldsymbol{\lambda}_{0}\right) \mathrm{e}^{K_{D i l}+b_{l}}\right)-\sum_{l=1}^{L} \frac{b_{l}^{2}}{2 B} .
\end{aligned}
$$

Observação: No caso da modelagem fixa, i.e. sem efeitos aleatórios, não são consideradas as distribuições a posteriori condicionais completas do vetor de efeitos aleatórios $\boldsymbol{b}$ e da variância do vetor de efeitos aleatórios $B$. Além disso, nas expressões para as demais distribuições a posteriori condicionais são retirados os efeitos aleatórios $b_{l}$ dado que agora tem-se o caso de indivíduos independentes.

Levando em conta que estas distribuições a posteriori condicionais não têm uma forma fechada, cada uma delas é amostrada pelo algoritmo de amostragem por fatias, proposto por Neal (2003), de tal forma que amostras da distribuição a posteriori aumentada (3.14) são obtidas implementado o amostrador por fatias das distribuições marginais em cada passo do amostrador de Gibbs.

Cabe resaltar aqui que este algoritmo não é o único que pode ser usado, outras possibilidades são o algoritmo de rejeição adapativa (ARS) proposto por Gilks e Wild (1992) ou o algoritmo de rejeição adaptativa de amostragem Metropolis (ARMS) de Gilks et al. (1995) se as densidades a posteriori condicionais completas possuem a propriedade de log-concavidade. Apesar de ter estudado esta propriedade para a maioria dos parâmetros, não é apresentada neste texto porque o algoritmo de amostragem escolhido (por fatias) não requer esta característica.

Para garantir que após várias iterações usando o algoritmo de Gibbs chega-se a uma distribuição estacionária, é importante estudar se a distribuição a posteriori completa (3.17) é própria.

\subsubsection{A distribuição a posteriori é própria?}

A distribuição a posteriori $\pi\left(\boldsymbol{\xi}, B \mid D_{\text {obs }}\right)$ será própria se a distribuição a priori $\pi(\boldsymbol{\xi}, B)$ for própria. Para um caso mais geral em que $\pi(\boldsymbol{\xi}, B)$ é imprópria ou inclusive se $\pi(\boldsymbol{\xi}, B)$ for vaga, $\pi\left(\boldsymbol{\xi}, B \mid D_{o b s}\right)$ será própria sobre certas condições, indicadas no 
teorema 1, enunciado a seguir.

Para avaliar a propriedade da distribuição a posteriori completa (3.12), é preciso definir as matrizes associadas aos preditores lineares do modelo e usar uma distribuição a priori imprópria. Para isso assume-se então $\pi(\boldsymbol{\alpha})=1, \pi\left(\beta_{0}, \gamma_{0}\right)=1, \pi\left(\beta_{1}, \gamma_{1}\right)=1$, $\pi\left(\boldsymbol{\beta}_{2}, \boldsymbol{\gamma}_{2}\right)=1$ e a prioris impróprias tipo Jeffreys para $\lambda_{m j}$, i.e, $\pi_{m \lambda}\left(\lambda_{m j}\right)=\frac{1}{\lambda_{m j}}$, $j=1, \cdots, J_{m}, m=0,1,2, \pi\left(\theta \mid \kappa_{\theta}, \varsigma_{\theta}\right)=\frac{\exp \left\{-\frac{\varsigma_{\theta}}{\theta}\right\}}{\theta^{\kappa} \theta^{+1}}$ e $\pi\left(B \mid \kappa_{B}, \varsigma_{B}\right)=\frac{\exp \left\{-\frac{\varsigma_{B}}{B}\right\}}{B^{\kappa} B^{+1}}$. Assim, a distribuição a priori de $(\boldsymbol{\xi}, B)$ imprópria conveniente é dada por

$$
\pi^{*}(\boldsymbol{\xi}, B) \propto \prod_{j=1}^{J_{0}} \frac{1}{\lambda_{0 j}} \prod_{j=1}^{J_{1}} \frac{1}{\lambda_{1 j}} \prod_{j=1}^{J_{2}} \frac{1}{\lambda_{2 j}} \frac{\exp \left\{-\frac{\varsigma_{\theta}}{\theta}\right\}}{\theta^{\kappa_{\theta}+1}} \frac{\exp \left\{-\frac{\varsigma_{B}}{B}\right\}}{B^{\kappa_{B}+1}} .
$$

Sejam $\mathcal{N}_{j}$ os conjuntos formados pelos indivíduos observados no caso $j$ para $j=$ $1,2,3,4$ e $n_{j}$ o cardinal de $\mathcal{N}_{j}, n_{j}=\left|\mathcal{N}_{j}\right|$. Considere-se também as matrizes:

- $\boldsymbol{X}_{E}$ de ordem $\left(n_{1}+n_{2}+n_{3}\right) \times p_{a}$ formada pelas linhas $\boldsymbol{x}_{E i l}=\left(1, A_{i l}, \boldsymbol{x}_{i l}^{\top}\right), \forall l$. Seja também $\boldsymbol{X}_{E}^{*}$, uma matriz da mesma ordem com linhas $w_{i l} \boldsymbol{x}_{E i l}=w_{i l}\left(1, A_{i l}, \boldsymbol{x}_{i l}^{\top}\right)$, em que

$w_{i l}= \begin{cases}-1, & \text { se } E_{i l}=1, \\ 1, & \text { se } E_{i l}=0 .\end{cases}$

- $\boldsymbol{X}_{0}$ de ordem $n_{1} \times\left(J_{0}+p_{0}\right)$ formada com linhas $\left(\delta_{i l 01}, \cdots, \delta_{i l 0 J_{0}}, A_{i l}, \boldsymbol{x}_{i l}^{\top}\right), i \in \mathcal{N}_{1}$ e $\forall l$, em que

$\delta_{i l 0 j}=\left\{\begin{array}{ll}1, & \text { se } y_{i l} \in\left(s_{0, j-1}, s_{0 j}\right] \\ 0 & \text { c.c. }\end{array}, j=1, \cdots, J_{0}, i \in \mathcal{N}_{1}\right.$ e $l=1, \cdots, L$.

- $\boldsymbol{X}_{1}$ de ordem $\left(n_{2}+n_{3}\right) \times\left(J_{1}+p_{1}\right)$ composta com linhas $\left(\delta_{i l 11}, \cdots, \delta_{i l 1 J_{1}}, A_{i l}, \boldsymbol{x}_{i l}^{\top}\right)$ para $i \in \mathcal{N}_{2} \cup \mathcal{N}_{3}, \forall l$ e sendo $\delta_{i l 1 j}=\left\{\begin{array}{ll}1, & \text { se } y_{E i l} \in\left(s_{1, j-1}, s_{1 j}\right] \\ 0, & \text { c.c. }\end{array}, j=1, \cdots, J_{1}\right.$, $i \in \mathcal{N}_{2} \cup \mathcal{N}_{3}$ e $l=1, \cdots, L$

- $\boldsymbol{X}_{2}$ de ordem $n_{2} \times\left(J_{2}+p_{2}\right)$ formada pelas linhas $\left(\delta_{i l 21}, \cdots, \delta_{i l 2 J_{2}}, A_{i l}, V_{i l}(1-\right.$ $\left.\left.A_{i l}\right), \boldsymbol{z}_{i l}^{\top}\right)$ para $i \in \mathcal{N}_{2}, \forall l$ e $\delta_{i l 2 j}= \begin{cases}1, & \text { se } y_{G i l}=y_{i l}-y_{E i l} \in\left(s_{2, j-1}, s_{2 j}\right] \\ 0, & \text { c.c. }\end{cases}$ $1, \cdots, J_{2}, i \in \mathcal{N}_{2}$ e $l=1, \cdots, L$.

Assume-se que as partições das funções de risco têm a propriedade que em cada intervalo da partição, $\left(s_{k, j-1}, s_{k j}\right] k=0,1,2$, é observado pelo menos um evento de interesse.

Com estas definições, estabelece-se o seguinte teorema que garante que a distribuição posteriori $\pi^{*}\left(\boldsymbol{\xi}, B \mid D_{o b s}\right)=\mathrm{L}\left(\boldsymbol{\xi}, B \mid D_{o b s}\right) \pi^{*}(\boldsymbol{\xi}, B)$ é própria.

Teorema 1 Assumindo o modelo (3.11) e a distribuição a priori (3.23), se as seguintes condições

(C1) a matriz de planejamento correspondente ao status da progressão $\boldsymbol{X}_{E}$ é de posto completo, 
(C2) existe um vetor com componentes positivas $c_{i}>0, \boldsymbol{c}=\left(c_{1}, \cdots, c_{n}^{*}\right)^{\top} \in \mathbb{R}^{n_{1}+n_{2}+n_{3}}$, tal que

$$
\boldsymbol{X}_{E}^{* \top} \boldsymbol{c}=\mathbf{0}
$$

(C3) $\int_{-\infty}^{\infty}|u|^{k} d F(u)<\infty$, com $F(\cdot)$ denotando uma função de ligação;

(C4) as matrizes $\boldsymbol{X}_{0}, \boldsymbol{X}_{1}$ e $\boldsymbol{X}_{2}$ são de posto completo $e$

(C5) $\kappa_{\theta}>0, \varsigma_{\theta}>0, \kappa_{B}>0, \varsigma_{B}>0$.

são satisfeitas, então a distribuição a posteriori $\pi^{*}\left(\boldsymbol{\xi}, B \mid D_{o b s}\right)=\mathrm{L}\left(\boldsymbol{\xi}, B \mid D_{o b s}\right) \pi^{*}(\boldsymbol{\xi}, B)$ é própria, i.e.

$$
\int_{R^{\boldsymbol{\xi}}} \mathrm{L}\left(\boldsymbol{\xi}, B \mid D_{o b s}\right) \pi^{*}(\boldsymbol{\xi}, B) d \boldsymbol{\xi} d B<\infty
$$

Resumindo, as condições C1 e C4 do teorema 1 são importantes porque as transformações lineares um a um que precisam ser feitas fornecem um jacobiano diferente de 0. As condições C2 e C3 são necessárias para obter a convergência da componente logito e a condição C5 está associada aos hiperparâmetros das distribuições a priori do parâmetro da cópula e da variância do efeito aleatório. Em conjunto, estas condições garantem a disitruibuição a posteriori ser própria

Inicialmente foi estudado este teorema para o modelo fixo (sem efeitos aleatórios) por ser mais simples, e em seguida, foi feita adaptação ao modelo misto. A prova do teorema para o modelo misto é apresentada no Apêndice B, considerando a modelagem com a cópula de Clayton. A prova para o modelo fixo não é apresentada nesta tese, pois em linhas gerais é bastante parecida com a prova para o modelo misto.

\subsection{Cópula de Frank}

Inicialmente, vale relembrar que, como descrito na Seção 2.2.3, a cópula de Frank é definida como

$$
\mathcal{C}_{\theta}(u, v)=-\frac{1}{\theta} \ln \left(1+\frac{\left(\mathrm{e}^{-\theta u}-1\right)\left(\mathrm{e}^{-\theta v}-1\right)}{\mathrm{e}^{-\theta}-1}\right), \quad \theta \in \mathbb{R} .
$$

e, sendo

$$
\begin{gathered}
u=S_{E}\left(t_{E} \mid A, \boldsymbol{x}, E=1, b\right)=\exp \left\{-H_{1}\left(t_{E}\right) \exp \left\{K_{E}+b\right\}\right\} \mathrm{e} \\
v=S_{G}\left(t_{G} \mid A, V, \boldsymbol{z}, E=1, b\right)=\exp \left\{-H_{2}\left(t_{G}\right) \exp \left\{K_{G}+b\right\}\right\},
\end{gathered}
$$

a função de sobrevivência conjunta dos tempos $T_{E}$ e $T_{G}$ para a componente do modelo (3.3) é dada por

$$
\begin{aligned}
& S_{E G_{\theta}}\left(t_{E}, t_{G} \mid A, \boldsymbol{x}, b\right)= \\
& \quad \frac{1}{\theta} \ln \left(1+\frac{\left(\mathrm{e}^{-\theta \exp \left\{-H_{1}\left(t_{E}\right) \exp \left\{K_{E}+b\right\}\right\}}-1\right)\left(\mathrm{e}^{-\theta \exp \left\{-H_{2}\left(t_{G}\right) \exp \left\{K_{G}+b\right\}\right\}}-1\right)}{\mathrm{e}^{-\theta}-1}\right) .
\end{aligned}
$$


A função de densidade bivariada fica dada por

$$
\begin{aligned}
& f_{E G_{\theta}}\left(t_{E}, t_{G} \mid A, V, \boldsymbol{x}, \boldsymbol{z}, b\right)= \\
& \quad h_{1}\left(t_{E}\right) \exp \left\{K_{E}+b-H_{1}\left(t_{E}\right) \mathrm{e}^{K_{E}+b}\right\} h_{2}\left(t_{G}\right) \exp \left\{K_{G}+b-H_{2}\left(t_{G}\right) \mathrm{e}^{K_{G}+b}\right\} \\
& \quad \times \frac{\theta\left(1-\mathrm{e}^{-\theta}\right) \exp \left\{-\theta\left(\exp \left\{H_{1}\left(t_{E}\right) \mathrm{e}^{K_{E}+b}\right\}+\exp \left\{H_{2}\left(t_{G}\right) \mathrm{e}^{K_{G}+b}\right\}\right)\right\}}{\left[\mathrm{e}^{-\theta}-1+\left(\exp \left\{-\theta \mathrm{e}^{-H_{1}\left(t_{E}\right) \mathrm{e}^{K_{E}+b}}\right\}-1\right)\left(\exp \left\{-\theta \mathrm{e}^{-H_{2}\left(t_{G}\right) \mathrm{e}^{K_{G}+b}}\right\}-1\right)\right]^{2}} .
\end{aligned}
$$

Considerando as funções (3.25) e (3.26) e os dados observados do indíviduo $i$ que pertence ao grupo $l$, a seguir obtem-se as expressões de cada caso para a construção da função de verossimilhança (3.11):

$$
\begin{aligned}
& \text { - } L_{1 i l}\left(\boldsymbol{\alpha}, \beta_{0}, \gamma_{0}, \boldsymbol{\lambda}_{0} \mid D_{i l}, b_{l}\right)=\frac{1}{1+\mathrm{e}^{K_{P i l}}} h_{0}\left(y_{i l} \mid \boldsymbol{\lambda}_{0}\right) \mathrm{e}^{K_{D i l}+b_{l}} \exp \left\{-H_{0}\left(y_{i l} \mid \boldsymbol{\lambda}_{0}\right) \mathrm{e}^{K_{D i l}+b_{l}}\right\}, \\
& \text { - } L_{2 i l}\left(\boldsymbol{\alpha}, \beta_{1}, \boldsymbol{\gamma}_{1}, \boldsymbol{\lambda}_{1}, \boldsymbol{\beta}_{2}, \boldsymbol{\gamma}_{2}, \boldsymbol{\lambda}_{2}, \theta \mid D_{i l}, b_{l}\right)=\frac{\mathrm{e}^{K_{P i l}}}{1+\mathrm{e}^{K_{P i l}}} h_{1}\left(y_{E i l} \mid \boldsymbol{\lambda}_{1}\right) \\
& \times \exp \left\{K_{E i l}+b_{l}-H_{1}\left(y_{E i l} \mid \boldsymbol{\lambda}_{1}\right) \mathrm{e}^{K_{E i l}+b_{l}}\right\} h_{2}\left(y_{G i l} \mid \boldsymbol{\lambda}_{2}\right) \\
& \times \exp \left\{K_{\text {Gil }}+b_{l}-H_{2}\left(y_{G i l} \mid \boldsymbol{\lambda}_{2}\right) \mathrm{e}^{K_{G i l}+b_{l}}\right\} \\
& \times \frac{\theta\left(1-\mathrm{e}^{-\theta}\right) \exp \left\{-\theta\left(\mathrm{e}^{-H_{1}\left(y_{E i l} \mid \boldsymbol{\lambda}_{1}\right) \mathrm{e}^{K_{E i l}+b_{l}}}+\mathrm{e}^{-H_{2}\left(y_{G i l} \mid \boldsymbol{\lambda}_{2}\right) \mathrm{e}^{K_{G i l}+b_{l}}}\right)\right\}}{\left[\mathrm{e}^{-\theta}-1+\left(\exp \left\{-\theta \mathrm{e}^{-H_{1}\left(y_{E i l} \mid \boldsymbol{\lambda}_{1}\right) \mathrm{e}^{K_{E i l}+b_{l}}}\right\}-1\right)\left(\exp \left\{-\theta \mathrm{e}^{-H_{2}\left(y_{G i l} \mid \boldsymbol{\lambda}_{2}\right) \mathrm{e}^{K_{G i l}+b_{l}}}\right\}-1\right)\right]^{2}}, \\
& \text { - } L_{3 i l}\left(\boldsymbol{\alpha}, \beta_{1}, \boldsymbol{\gamma}_{1}, \boldsymbol{\lambda}_{1}, \boldsymbol{\beta}_{2}, \boldsymbol{\gamma}_{2}, \boldsymbol{\lambda}_{2}, \theta \mid D_{i l}, b_{l}\right)=\frac{\mathrm{e}^{K_{P i l}}}{1+\mathrm{e}^{K_{P i l}}} h_{1}\left(y_{E i l} \mid \boldsymbol{\lambda}_{1}\right) \\
& \times \exp \left\{K_{E i l}+b_{l}-H_{1}\left(y_{E i l} \mid \boldsymbol{\lambda}_{1}\right) \mathrm{e}^{K_{E}+b}\right\} \exp \left\{-\theta \mathrm{e}^{-H_{1}\left(y_{E i l} \mid \boldsymbol{\lambda}_{1}\right) \mathrm{e}^{K_{E}+b}}\right\} \\
& \times\left(\exp \left\{-\theta \mathrm{e}^{-H_{2}\left(y_{G i l} \mid \boldsymbol{\lambda}_{2}\right) \mathrm{e}^{K_{G i l}+b_{l}}}\right\}-1\right) \\
& \times\left[\mathrm{e}^{-\theta}+\exp \left\{-\theta\left(\mathrm{e}^{-H_{1}\left(y_{E i l} \mid \boldsymbol{\lambda}_{1}\right) \mathrm{e}^{K_{E i l}+b_{l}}}+\mathrm{e}^{-H_{2}\left(y_{G i l} \mid \boldsymbol{\lambda}_{2}\right) \mathrm{e}^{K_{G i l}+b_{l}}}\right)\right\}\right. \\
& \left.-\exp \left\{-\theta \mathrm{e}^{-H_{1}\left(y_{E i l} \mid \boldsymbol{\lambda}_{1}\right) \mathrm{e}^{K_{E i l}+b_{l}}}\right\}-\exp \left\{-\theta \mathrm{e}^{-H_{2}\left(y_{G i l} \mid \boldsymbol{\lambda}_{2}\right) \mathrm{e}^{K_{G i l}+b_{l}}}\right\}\right]^{-1} \text {; }
\end{aligned}
$$

e

$$
\begin{aligned}
& -L_{4 i}\left(\boldsymbol{\alpha}, \beta_{0}, \boldsymbol{\gamma}_{0}, \boldsymbol{\lambda}_{0}, \beta_{1}, \boldsymbol{\gamma}_{1}, \boldsymbol{\lambda}_{1}, \theta \mid D_{i l}, b_{l}\right)= \\
& \quad \frac{\mathrm{e}^{K_{P} i l}}{1+\mathrm{e}^{K_{P} i l}} \exp \left\{-H_{1}\left(y_{i l} \mid \boldsymbol{\lambda}_{1}\right) \mathrm{e}^{K_{E i l}+b_{l}}\right\}+\frac{1}{1+\mathrm{e}^{K_{P} i l}} \exp \left\{-H_{0}\left(y_{i l} \mid \boldsymbol{\lambda}_{0}\right) \mathrm{e}^{K_{D i l}+b_{l}}\right\} .
\end{aligned}
$$

Observe que as expressões para os casos um e quatro são independentes da cópula, ou seja, elas se conservam para qualquer cópula.

Levando em conta que o espaço paramétrico de $\theta$ para a cópula de Frank é $\theta \in \mathbb{R}$, em este caso é usada um distribuição normal com média 0 e variância $\sigma_{\theta}^{2}$ para a distribuição a priori de $\theta$ (Romeo et al., 2006). 
Conservando o mesmo raciocínio utilizado com a cópula de Clayton, a distribuição a posteriori completa aumentada (3.14) segundo a cópula de Frank é dada por

$$
\begin{aligned}
& \pi\left(\boldsymbol{\xi}, B, \boldsymbol{b}, \boldsymbol{E}^{*} \mid D_{o b s}\right) \propto \\
& \left(\prod_{l=1}^{L} \prod_{i=1}^{n_{l}}\left[\frac{1}{1+\mathrm{e}^{K_{P i l}}} h_{0}\left(y_{i l} \mid \boldsymbol{\lambda}_{0}\right) \mathrm{e}^{K_{D i l}+b_{l}} \exp \left\{-H_{0}\left(y_{i l} \mid \boldsymbol{\lambda}_{0}\right) \mathrm{e}^{K_{D i l}+b_{l}}\right\}\right]^{\mathbb{I}\left\{\begin{array}{l}
d_{i l}=0 \\
v_{i l}=1
\end{array}\right\}}\right. \\
& \times\left[\frac{\mathrm{e}^{K_{P i l}}}{1+\mathrm{e}^{K_{\text {Pil }}}} h_{1}\left(y_{E i l} \mid \boldsymbol{\lambda}_{1}\right) \exp \left\{K_{E i l}+b_{l}-H_{1}\left(y_{E i l} \mid \boldsymbol{\lambda}_{1}\right) \mathrm{e}^{K_{E i l}+b_{l}}\right\}\right. \\
& \times h_{2}\left(y_{G i l} \mid \boldsymbol{\lambda}_{2}\right) \exp \left\{K_{G i l}+b_{l}-H_{2}\left(y_{G i l} \mid \boldsymbol{\lambda}_{2}\right) \mathrm{e}^{K_{G i l}+b_{l}}\right\} \\
& \left.\times \frac{\theta\left(1-\mathrm{e}^{-\theta}\right) \exp \left\{-\theta\left(\mathrm{e}^{-H_{1}\left(y_{E i l} \mid \boldsymbol{\lambda}_{1}\right) \mathrm{e}^{K_{E i l}+b_{l}}}+\mathrm{e}^{-H_{2}\left(y_{G i l} \mid \boldsymbol{\lambda}_{2}\right) \mathrm{e}^{K_{G i l}+b_{l}}}\right)\right\}}{\left[\mathrm{e}^{-\theta}-1+\left(\exp \left\{-\theta \mathrm{e}^{-H_{1}\left(y_{E i l} \mid \boldsymbol{\lambda}_{1}\right) \mathrm{e}^{K_{E i l}+b_{l}}}\right\}-1\right)\left(\exp \left\{-\theta \mathrm{e}^{-H_{2}\left(y_{G i l} \mid \boldsymbol{\lambda}_{2}\right) \mathrm{e}^{K_{G i l}+b_{l}}}\right\}-1\right)\right]^{2}}\right]^{\mathbb{I}\left\{\begin{array}{c}
d_{i l l}=1 \\
v_{i l}=1
\end{array}\right\}} \\
& \times\left[\frac{\mathrm{e}^{K_{P i l}}}{1+\mathrm{e}^{K_{P i l}}} h_{1}\left(y_{E i l} \mid \boldsymbol{\lambda}_{1}\right) \exp \left\{K_{E i l}+b_{l}-H_{1}\left(y_{E i l} \mid \boldsymbol{\lambda}_{1}\right) \mathrm{e}^{K_{E i l}+b_{l}}\right\}\right. \\
& \times \exp \left\{-\theta \mathrm{e}^{-H_{1}\left(y_{E i l} \mid \boldsymbol{\lambda}_{1}\right) \mathrm{e}^{K_{E i l}+b_{l}}}\right\}\left(\exp \left\{-\theta \mathrm{e}^{-H_{2}\left(y_{G i l} \mid \boldsymbol{\lambda}_{2}\right) \mathrm{e}^{K_{G i l}+b_{l}}}\right\}-1\right) \\
& \times\left[\mathrm{e}^{-\theta}+\exp \left\{-\theta\left(\mathrm{e}^{-H_{1}\left(y_{E i l} \mid \boldsymbol{\lambda}_{1}\right) \mathrm{e}^{K_{E i l}+b_{l}}}+\mathrm{e}^{-H_{2}\left(y_{G i l} \mid \boldsymbol{\lambda}_{2}\right) \mathrm{e}^{K_{G i l}+b_{l}}}\right)\right\}\right. \\
& \left.\left.-\exp \left\{-\theta \mathrm{e}^{-H_{1}\left(y_{E i l} \mid \boldsymbol{\lambda}_{1}\right) \mathrm{e}^{K_{E i l}+b_{l}}}\right\}-\exp \left\{-\theta \mathrm{e}^{-H_{2}\left(y_{G i l} \mid \boldsymbol{\lambda}_{2}\right) \mathrm{e}^{K_{G i l}+b_{l}}}\right\}\right]^{-1}\right]^{\mathbb{I}\left\{\begin{array}{l}
d_{i l}=1 \\
v_{i l}=0
\end{array}\right.} \\
& \times\left[\left(\frac{\mathrm{e}^{K_{P} i l}}{1+\mathrm{e}^{K_{P} i l}} \exp \left\{-H_{1}\left(y_{i l} \mid \boldsymbol{\lambda}_{1}\right) \mathrm{e}^{K_{E i l}+b_{l}}\right\}\right)^{E_{i l}^{*}}\right. \\
& \left.\left.\times\left(\frac{1}{1+\mathrm{e}^{K_{P} i l}} \exp \left\{-H_{0}\left(y_{i l} \mid \boldsymbol{\lambda}_{0}\right) \mathrm{e}^{K_{D i l}+b_{l}}\right\}\right)^{1-E_{i l}^{*}}\right]^{\mathbb{I}\left\{\begin{array}{l}
d_{i l}=0 \\
v_{i l}=0
\end{array}\right.}(2 \pi B)^{-\frac{1}{2}} \exp \left\{-\frac{b_{l}^{2}}{2 B}\right\}\right) \\
& \times\left(\exp \left\{-0,5\left(\alpha_{0}, \alpha_{1}, \boldsymbol{\alpha}_{2}^{\top}\right)^{\top} \boldsymbol{\Sigma}_{\alpha}^{-1}\left(\alpha_{0}, \alpha_{1}, \boldsymbol{\alpha}_{2}^{\top}\right)\right\} \exp \left\{-0,5\left(\beta_{0}, \boldsymbol{\gamma}_{0}^{\top}\right)^{\top} \boldsymbol{\Sigma}_{0}^{-1}\left(\beta_{0}, \boldsymbol{\gamma}_{0}^{\top}\right)\right\}\right. \\
& \times \exp \left\{-0,5\left(\beta_{1}, \boldsymbol{\gamma}_{1}^{\top}\right)^{\top} \boldsymbol{\Sigma}_{1}^{-1}\left(\beta_{1}, \boldsymbol{\gamma}_{1}^{\top}\right)\right\} \exp \left\{-0,5\left(\boldsymbol{\beta}_{2}^{\top}, \boldsymbol{\gamma}_{2}^{\top}\right)^{\top} \boldsymbol{\Sigma}_{2}^{-1}\left(\boldsymbol{\beta}_{\mathbf{2}}^{\top}, \boldsymbol{\gamma}_{2}^{\top}\right)\right\} \prod_{j=1}^{J 0} \lambda_{0 j}^{a_{0 j}-1} \\
& \times \exp \left\{-b_{0 j} \lambda_{0 j}\right\} \prod_{j=1}^{J 1} \lambda_{1 j}^{a_{1 j}-1} \exp \left\{-b_{1 j} \lambda_{1 j}\right\} \prod_{j=1}^{J 2} \lambda_{2 j}^{a_{2 j}-1} \exp \left\{-b_{2 j} \lambda_{2 j}\right\} \exp \left\{-\frac{\theta^{2}}{2 \sigma_{\theta}^{2}}\right\} \\
& \left.\times\left(\frac{1}{B}\right)^{\kappa_{B}+1} \exp \left\{-\frac{\varsigma_{B}}{B}\right\}\right) \text {. }
\end{aligned}
$$

A seguir são obtidas as distribuições condicionais completas de cada parâmetro dado os demais parâmetros:

1. Distribuição a posteriori condicional completa de $\boldsymbol{\lambda}_{0}$

Esta distribuição se conserva como descrita na cópula de Clayton porque $\boldsymbol{\lambda}_{0}$ está associado aos casos 1 e 4, em que a contribuição à função de verossimilhança é a mesma independentemente da cópula. 
2. Distribuição a posteriori condicional completa de $\boldsymbol{\lambda}_{1}$

De (3.27) pode se observar que a distribuição a posteriori condicional completa de $\boldsymbol{\lambda}_{1}$ é

$$
\begin{aligned}
& \pi\left(\boldsymbol{\lambda}_{1} \mid \beta_{1}, \boldsymbol{\gamma}_{1}, \boldsymbol{\beta}_{2}, \boldsymbol{\gamma}_{2}, \boldsymbol{\lambda}_{2}, \theta, \boldsymbol{b}, \boldsymbol{E}^{*}, D_{o b s}\right) \propto \prod_{l=1}^{L} \prod_{i=1}^{n_{l}} \\
& \times\left[\frac{h_{1}\left(y_{E i l} \mid \boldsymbol{\lambda}_{1}\right) \exp \left\{-H_{1}\left(y_{E i l} \mid \boldsymbol{\lambda}_{1}\right) \mathrm{e}^{K_{E i l}+b_{l}}-\theta \mathrm{e}^{-H_{1}\left(y_{E i l} \mid \boldsymbol{\lambda}_{1}\right) \mathrm{e}^{K_{E i l}+b_{l}}}\right\}}{\left[\mathrm{e}^{-\theta}-1+\left(\exp \left\{-\theta \mathrm{e}^{-H_{1}\left(y_{E i l} \mid \boldsymbol{\lambda}_{1}\right) \mathrm{e}^{K_{E i l}+b_{l}}}\right\}-1\right)\left(\exp \left\{-\theta \mathrm{e}^{-H_{2}\left(y_{G i l} \mid \boldsymbol{\lambda}_{2}\right) \mathrm{e}^{K_{G i l}+b_{l}}}\right\}-1\right)\right]^{2}}\right]^{\mathbb{I}\left\{\begin{array}{c}
d_{i l}=1 \\
v_{i l}=1
\end{array}\right\}} \\
& \times\left[h_{1}\left(y_{E i l} \mid \boldsymbol{\lambda}_{1}\right) \exp \left\{-H_{1}\left(y_{E i l} \mid \boldsymbol{\lambda}_{1}\right) \mathrm{e}^{K_{E i l}+b_{l}}-\theta \mathrm{e}^{-H_{1}\left(y_{E i l} \mid \boldsymbol{\lambda}_{1}\right) \mathrm{e}^{K_{E i l}+b_{l}}}\right\}\right. \\
& \times\left[\mathrm{e}^{-\theta}+\exp \left\{-\theta\left(\mathrm{e}^{-H_{1}\left(y_{E i l} \mid \boldsymbol{\lambda}_{1}\right) \mathrm{e}^{K_{E i l}+b_{l}}}+\mathrm{e}^{-H_{2}\left(y_{G i l} \mid \boldsymbol{\lambda}_{2}\right) \mathrm{e}^{K_{G i l}+b_{l}}}\right)\right\}\right. \\
& \left.\left.-\exp \left\{-\theta \mathrm{e}^{-H_{1}\left(y_{E i l} \mid \boldsymbol{\lambda}_{1}\right) \mathrm{e}^{K_{E i l}+b_{l}}}\right\}-\exp \left\{-\theta \mathrm{e}^{-H_{2}\left(y_{G i l} \mid \boldsymbol{\lambda}_{2}\right) \mathrm{e}^{K_{G i l}+b_{l}}}\right\}\right]^{-1}\right]^{\mathbb{I}\left\{\begin{array}{c}
d_{i l}=1 \\
v_{i l}=0
\end{array}\right\}} \\
& \times \exp \left\{-E_{i l}^{*} \mathbb{I}\left\{\begin{array}{c}
d_{i l}=0 \\
v_{i l}=0
\end{array}\right\} H_{1}\left(y_{i l} \mid \boldsymbol{\lambda}_{1}\right) \mathrm{e}^{K_{E i l}+b_{l}}\right\} \prod_{j=1}^{J 1} \lambda_{1 j}^{a_{1 j}-1} \exp \left\{-b_{1 j} \lambda_{1 j}\right\},
\end{aligned}
$$

sendo que a função log-posteriori é dada por

$$
\begin{aligned}
& \mathcal{L}\left(\pi\left(\boldsymbol{\lambda}_{1} \mid \beta_{1}, \boldsymbol{\gamma}_{1}, \boldsymbol{\beta}_{2}, \boldsymbol{\gamma}_{2}, \boldsymbol{\lambda}_{2}, \theta, \boldsymbol{b}, \boldsymbol{E}^{*}, D_{o b s}\right)\right)=\sum_{l=1}^{L} \sum_{i=1}^{n_{l}}\left(\mathbb{I}\left\{\begin{array}{c}
d_{i l}=1 \\
v_{i l}=1
\end{array}\right\}+\mathbb{I}\left\{\begin{array}{l}
d_{i l}=1 \\
v_{i l}=0
\end{array}\right\}\right) \\
& \times\left(\ln \left(h_{1}\left(y_{E i l} \mid \boldsymbol{\lambda}_{1}\right)\right)-H_{1}\left(y_{E i l} \mid \boldsymbol{\lambda}_{1}\right) \mathrm{e}^{K_{E i l}+b_{l}}-\theta \mathrm{e}^{-H_{1}\left(y_{E i l} \mid \boldsymbol{\lambda}_{1}\right) \mathrm{e}^{K_{E i l}+b_{l}}}\right) \\
& -\sum_{l=1}^{L} \sum_{i=1}^{n_{l}} 2 \mathbb{I}\left\{\begin{array}{l}
d_{i l}=1 \\
v_{i l}=1
\end{array}\right\} \ln \left(\mathrm{e}^{-\theta}-1+\left(\exp \left\{-\theta \mathrm{e}^{-H_{1}\left(y_{E i l} \mid \boldsymbol{\lambda}_{1}\right) \mathrm{e}^{K_{E i l}+b_{l}}}\right\}-1\right)\right. \\
& \left.\times\left(\exp \left\{-\theta \mathrm{e}^{-H_{2}\left(y_{G i l} \mid \boldsymbol{\lambda}_{2}\right) \mathrm{e}^{K_{G i l}+b_{l}}}\right\}-1\right)\right)-\sum_{l=1}^{L} \sum_{i=1}^{n_{l}} \mathbb{I}\left\{\begin{array}{l}
d_{i l}=1 \\
v_{i l}=0
\end{array}\right\} \ln \left(\mathrm{e}^{-\theta}\right. \\
& +\exp \left\{-\theta\left(\mathrm{e}^{-H_{1}\left(y_{E i l} \mid \boldsymbol{\lambda}_{1}\right) \mathrm{e}^{K_{E i l}+b_{l}}}+\mathrm{e}^{-H_{2}\left(y_{G i l} \mid \boldsymbol{\lambda}_{2}\right) \mathrm{e}^{K_{G i l}+b_{l}}}\right)\right\} \\
& \left.-\exp \left\{-\theta \mathrm{e}^{-H_{1}\left(y_{E i l} \mid \boldsymbol{\lambda}_{1}\right) \mathrm{e}^{K_{E i l}+b_{l}}}\right\}-\exp \left\{-\theta \mathrm{e}^{-H_{2}\left(y_{G i l} \mid \boldsymbol{\lambda}_{2}\right) \mathrm{e}^{K_{G i l}+b_{l}}}\right\}\right) \\
& -\sum_{l=1}^{L} \sum_{i=1}^{n_{l}} E_{i l}^{*} \mathbb{I}\left\{\begin{array}{l}
d_{i l}=0 \\
v_{i l}=0
\end{array}\right\} H_{1}\left(y_{i l} \mid \boldsymbol{\lambda}_{1}\right) \mathrm{e}^{K_{E i l}+b_{l}}+\sum_{j=1}^{J 1}\left(a_{1 j}-1\right) \log \left(\lambda_{1 j}\right)-b_{1 j} \lambda_{1 j} \text {. }
\end{aligned}
$$

3. Distribuição a posteriori condicional completa de $\boldsymbol{\lambda}_{2}$

De (3.27) obtemos a distribuição a posteriori condicional completa de $\boldsymbol{\lambda}_{2}$ que é dada por 


$$
\begin{aligned}
& \pi\left(\boldsymbol{\lambda}_{2} \mid \beta_{1}, \boldsymbol{\gamma}_{1}, \boldsymbol{\lambda}_{1}, \boldsymbol{\beta}_{2}, \boldsymbol{\gamma}_{2}, \theta, \boldsymbol{b}, D_{o b s}\right) \propto \prod_{l=1}^{L} \prod_{i=1}^{n_{l}}
\end{aligned}
$$

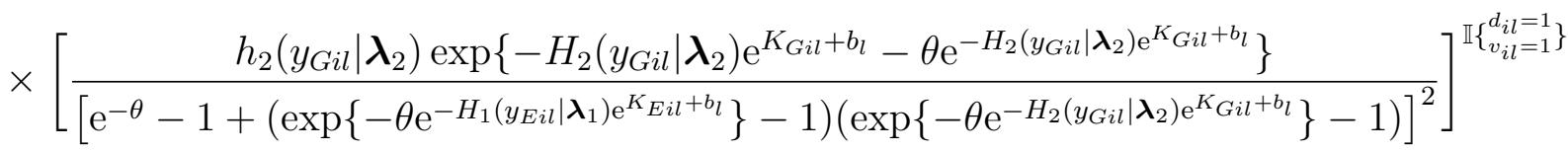

$$
\begin{aligned}
& \times\left[( \operatorname { e x p } \{ - \theta \mathrm { e } ^ { - H _ { 2 } ( y _ { G i l } | \boldsymbol { \lambda } _ { 2 } ) \mathrm { e } ^ { K _ { G i l } + b _ { l } } } \} - 1 ) \left[\mathrm{e}^{-\theta}\right.\right. \\
& +\exp \left\{-\theta\left(\mathrm{e}^{-H_{1}\left(y_{E i l} \mid \boldsymbol{\lambda}_{1}\right) \mathrm{e}^{K_{E i l}+b_{l}}}+\mathrm{e}^{-H_{2}\left(y_{G i l} \mid \boldsymbol{\lambda}_{2}\right) \mathrm{e}^{K_{G i l}+b_{l}}}\right)\right\} \exp \left\{-\theta \mathrm{e}^{-H_{1}\left(y_{E i l} \mid \boldsymbol{\lambda}_{1}\right) \mathrm{e}^{K_{E i l}+b_{l}}}\right\} \\
& \left.\left.\left.-\exp \left\{-\theta \mathrm{e}^{-H_{2}\left(y_{G i l} \mid \boldsymbol{\lambda}_{2}\right) \mathrm{e}^{K_{G i l}+b_{l}}}\right\}\right]^{-1}\right]^{\mathbb{I}\left\{v_{i l}^{d_{i l}=1}=0\right.}\right\} \prod_{j=1}^{J 2} \lambda_{2 j}^{a_{2 j}-1} \exp \left\{-b_{2 j} \lambda_{2 j}\right\},
\end{aligned}
$$

em que a função log-posteriori é dada por

$$
\begin{aligned}
& \mathcal{L}\left(\pi\left[\boldsymbol{\lambda}_{2} \mid \beta_{1}, \boldsymbol{\gamma}_{1}, \boldsymbol{\lambda}_{1}, \boldsymbol{\beta}_{2}, \boldsymbol{\gamma}_{2}, \theta, \boldsymbol{b}, D_{o b s}\right]\right)=\sum_{l=1}^{L} \sum_{i=1}^{n_{l}} \mathbb{I}\left\{\begin{array}{l}
d_{i l}=1 \\
v_{i l}=1
\end{array}\right\}\left(\ln \left(h_{2}\left(y_{G i l} \mid \boldsymbol{\lambda}_{2}\right)\right)\right. \\
& -H_{2}\left(y_{G i l} \mid \boldsymbol{\lambda}_{2}\right) \mathrm{e}^{K_{G i l}+b_{l}}-\theta \mathrm{e}^{-H_{2}\left(y_{G i l} \mid \boldsymbol{\lambda}_{2}\right) \mathrm{e}^{K_{G i l}+b_{l}}}-2 \ln \left(\mathrm{e}^{-\theta}-1\right. \\
& \left.+\left(\exp \left\{-\theta \mathrm{e}^{-H_{1}\left(y_{E i l} \mid \boldsymbol{\lambda}_{1}\right) \mathrm{e}^{K_{E i l}+b_{l}}}\right\}-1\right)\left(\exp \left\{-\theta \mathrm{e}^{-H_{2}\left(y_{G i l} \mid \boldsymbol{\lambda}_{2}\right) \mathrm{e}^{K_{G i l}+b_{l}}}\right\}-1\right)\right) \\
& +\sum_{l=1}^{L} \sum_{i=1}^{n_{l}} \mathbb{I}\left\{\begin{array}{l}
d_{i l}=1 \\
v_{i l}=0
\end{array}\right\}\left(\ln \left(\exp \left\{-\theta \mathrm{e}^{-H_{2}\left(y_{G i l} \mid \boldsymbol{\lambda}_{2}\right) \mathrm{e}^{K_{G i l}+b_{l}}}\right\}-1\right)-\ln \left(\mathrm{e}^{-\theta}\right.\right. \\
& +\exp \left\{-\theta\left(\mathrm{e}^{-H_{1}\left(y_{E i l} \mid \boldsymbol{\lambda}_{1}\right) \mathrm{e}^{K_{E i l}+b_{l}}}+\mathrm{e}^{-H_{2}\left(y_{G i l} \mid \boldsymbol{\lambda}_{2}\right) \mathrm{e}^{K_{G i l}+b_{l}}}\right)\right\}-\exp \left\{-\theta \mathrm{e}^{-H_{1}\left(y_{E i l} \mid \boldsymbol{\lambda}_{1}\right) \mathrm{e}^{K_{E i l}+b_{l}}}\right\} \\
& \left.\left.-\exp \left\{-\theta \mathrm{e}^{-H_{2}\left(y_{G i l} \mid \boldsymbol{\lambda}_{2}\right) \mathrm{e}^{K_{G i l}+b_{l}}}\right\}\right)\right)+\sum_{j=1}^{J 2}\left(a_{2 j}-1\right) \ln \left(\lambda_{2 j}\right)-b_{2 j} \lambda_{2 j} .
\end{aligned}
$$

4. Distribuição a posteriori condicional completa de $\left(\beta_{0}, \gamma_{0}\right)$

A distribuição condicional completa de $\left(\beta_{0}, \gamma_{0}\right)$ é independente da cópula e, portanto, é a mesma expressão da cópula de Clayton dado por (3.20).

5. Distribuição a posteriori condicional completa de $\left(\beta_{1}, \gamma_{1}\right)$

Relembrando que $K_{E i l}=A_{i l} \beta_{1}+\boldsymbol{x}_{i l}^{\top} \boldsymbol{\gamma}_{1}$ é o preditor linear de efeitos fixos e pela independência entre parâmetros, observa-se de (3.27) que a distribição a posteriori condicional completa de $\left(\beta_{1}, \gamma_{1}\right)$ é expressa como 


$$
\begin{aligned}
& \pi\left(\beta_{1}, \gamma_{1} \mid \boldsymbol{\lambda}_{1}, \boldsymbol{\beta}_{2}, \boldsymbol{\gamma}_{2}, \boldsymbol{\lambda}_{2}, \theta, \boldsymbol{b}, \boldsymbol{E}^{*}, D_{o b s}\right) \propto \prod_{l=1}^{L} \prod_{i=1}^{n_{l}} \\
& \times\left[\frac{\exp \left\{K_{E i l}-H_{1}\left(y_{E i l} \mid \boldsymbol{\lambda}_{1}\right) \mathrm{e}^{K_{E i l}+b_{l}}-\theta \mathrm{e}^{-H_{1}\left(y_{E i l} \mid \boldsymbol{\lambda}_{1}\right) \mathrm{e}^{K_{E i l}+b_{l}}}\right\}}{\left[\mathrm{e}^{-\theta}-1+\left(\exp \left\{-\theta \mathrm{e}^{-H_{1}\left(y_{E i l} \mid \boldsymbol{\lambda}_{1}\right) \mathrm{e}^{K_{E i l}+b_{l}}}\right\}-1\right)\left(\exp \left\{-\theta \mathrm{e}^{-H_{2}\left(y_{G i l} \mid \boldsymbol{\lambda}_{2}\right) \mathrm{e}^{K_{G i l}+b_{l}}}\right\}-1\right)\right]^{2}}\right]^{\mathbb{I}\left\{\begin{array}{c}
d_{i l}=1 \\
v_{i l}=1
\end{array}\right\}} \\
& \times\left[\exp \left\{K_{E i l}-H_{1}\left(y_{E i l} \mid \boldsymbol{\lambda}_{1}\right) \mathrm{e}^{K_{E i l}+b}-\theta \mathrm{e}^{-H_{1}\left(y_{E i l} \mid \boldsymbol{\lambda}_{1}\right) \mathrm{e}^{K_{E i l}+b}}\right\}\right. \\
& \times\left[\mathrm{e}^{-\theta}+\exp \left\{-\theta\left(\mathrm{e}^{-H_{1}\left(y_{E i l} \mid \boldsymbol{\lambda}_{1}\right) \mathrm{e}^{K_{E i l}+b_{l}}}+\mathrm{e}^{-H_{2}\left(y_{G i l} \mid \boldsymbol{\lambda}_{2}\right) \mathrm{e}^{K_{G i l}+b_{l}}}\right)\right\}\right. \\
& \left.\left.-\exp \left\{-\theta \mathrm{e}^{-H_{1}\left(y_{E i l} \mid \boldsymbol{\lambda}_{1}\right) \mathrm{e}^{K_{E i l}+b_{l}}}\right\}-\exp \left\{-\theta \mathrm{e}^{-H_{2}\left(y_{G i l} \mid \boldsymbol{\lambda}_{2}\right) \mathrm{e}^{K_{G i l}+b_{l}}}\right\}\right]^{-1}\right]^{\mathbb{I}\left\{\begin{array}{c}
d_{i l}=1 \\
v_{i l}=0
\end{array}\right\}} \\
& \times \exp \left\{-E_{i l}^{*} \mathbb{I}\left\{\begin{array}{c}
d_{i l=0}=0 \\
v_{i l}=0
\end{array}\right\} H_{1}\left(y_{i l} \mid \boldsymbol{\lambda}_{1}\right) \mathrm{e}^{K_{E i l}+b_{l}}\right\} \exp \left\{-0,5\left(\beta_{1}, \boldsymbol{\gamma}_{1}^{\top}\right)^{\top} \boldsymbol{\Sigma}_{1}^{-1}\left(\beta_{1}, \boldsymbol{\gamma}_{1}^{\top}\right)\right\} \text {, }
\end{aligned}
$$

e a função log-posteriori é dada por

$$
\begin{aligned}
& \mathcal{L}\left(\pi\left(\beta_{1}, \boldsymbol{\gamma}_{1} \mid \boldsymbol{\lambda}_{1}, \boldsymbol{\beta}_{2}, \boldsymbol{\gamma}_{2}, \boldsymbol{\lambda}_{2}, \theta, \boldsymbol{b}, \boldsymbol{E}^{*}, D_{o b s}, b_{l}\right)\right)=\sum_{l=1}^{L} \sum_{i=1}^{n_{l}}\left(\mathbb{I}\left\{\begin{array}{l}
d_{i l}=1 \\
v_{i l}=1
\end{array}\right\}+\mathbb{I}\left\{\begin{array}{l}
d_{i l}=1 \\
v_{i l}=0
\end{array}\right\}\right) \\
& \times\left(K_{E i l}-H_{1}\left(y_{E i l} \mid \boldsymbol{\lambda}_{1}\right) \mathrm{e}^{K_{E i l}+b_{l}}-\theta \mathrm{e}^{-H_{1}\left(y_{E i l} \mid \boldsymbol{\lambda}_{1}\right) \mathrm{e}^{K_{E i l}+b_{l}}}\right) \\
& -\sum_{l=1}^{L} \sum_{i=1}^{n_{l}} 2 \mathbb{I}\left\{\begin{array}{c}
d_{i l}=1 \\
v_{i l}=1
\end{array}\right\} \ln \left(\mathrm{e}^{-\theta}-1+\left(\exp \left\{-\theta \mathrm{e}^{-H_{1}\left(y_{E i l} \mid \boldsymbol{\lambda}_{1}\right) \mathrm{e}^{K_{E i l}+b_{l}}}\right\}-1\right)\right. \\
& \left.\times\left(\exp \left\{-\theta \mathrm{e}^{-H_{2}\left(y_{G i l} \mid \boldsymbol{\lambda}_{2}\right) \mathrm{e}^{K_{G i l}+b_{l}}}\right\}-1\right)\right)-\sum_{l=1}^{L} \sum_{i=1}^{n_{l}} \mathbb{I}\left\{\begin{array}{l}
d_{i l}=1 \\
v_{i l}=0
\end{array}\right\} \ln \left(\mathrm{e}^{-\theta}\right. \\
& +\exp \left\{-\theta\left(\mathrm{e}^{-H_{1}\left(y_{E i l} \mid \boldsymbol{\lambda}_{1}\right) \mathrm{e}^{K_{E i l}+b_{l}}}+\mathrm{e}^{-H_{2}\left(y_{G i l} \mid \boldsymbol{\lambda}_{2}\right) \mathrm{e}^{K_{G i l}+b_{l}}}\right)\right\} \\
& \left.-\exp \left\{-\theta \mathrm{e}^{-H_{1}\left(y_{E i l} \mid \boldsymbol{\lambda}_{1}\right) \mathrm{e}^{K_{E i l}+b_{l}}}\right\}-\exp \left\{-\theta \mathrm{e}^{-H_{2}\left(y_{G i l} \mid \boldsymbol{\lambda}_{2}\right) \mathrm{e}^{K_{G i l}+b_{l}}}\right\}\right) \\
& -\sum_{l=1}^{L} \sum_{i=1}^{n_{l}} E_{i l}^{*} \mathbb{I}\left\{\begin{array}{c}
d_{i l}=0 \\
v_{i l}=0
\end{array}\right\} H_{1}\left(y_{i l} \mid \boldsymbol{\lambda}_{1}\right) \mathrm{e}^{K_{E i l}+b_{l}}-0,5\left(\beta_{1}, \boldsymbol{\gamma}_{1}^{\top}\right)^{\top} \boldsymbol{\Sigma}_{1}^{-1}\left(\beta_{1}, \boldsymbol{\gamma}_{1}^{\top}\right) \text {. }
\end{aligned}
$$

6. Distribuição a posteriori condicional completa de $\left(\beta_{2}, \gamma_{2}\right)$

Considerando o preditor linear dos efeitos fixos $K_{G i l}=A_{i l} \beta_{21}+V_{i l}\left(1-A_{i l}\right) \beta_{22}+$ $\boldsymbol{z}_{i l}^{\top} \boldsymbol{\gamma}_{2}$ e a independência entre parâmetros tem-se que de (3.27) a distribuição $a$ posteriori condicional completa de $\left(\boldsymbol{\beta}_{2}, \boldsymbol{\gamma}_{2}\right)$ é 


$$
\begin{aligned}
& \pi\left(\boldsymbol{\beta}_{2}, \boldsymbol{\gamma}_{2} \mid \beta_{1}, \boldsymbol{\gamma}_{1}, \boldsymbol{\lambda}_{1}, \boldsymbol{\lambda}_{2}, \theta, D_{o b s}, b_{l}\right) \propto \prod_{l=1}^{L} \prod_{i=1}^{n_{l}} \\
& {\left[\frac{\exp \left\{K_{G i l}-H_{2}\left(y_{G i l} \mid \boldsymbol{\lambda}_{2}\right) \mathrm{e}^{K_{G i l}+b_{l}}-\theta \mathrm{e}^{-H_{2}\left(y_{G i l} \mid \boldsymbol{\lambda}_{2}\right) \mathrm{e}^{K_{G i l}+b_{l}}}\right\}}{\left[\mathrm{e}^{-\theta}-1+\left(\exp \left\{-\theta \mathrm{e}^{-H_{1}\left(y_{E i l} \mid \boldsymbol{\lambda}_{1}\right) \mathrm{e}^{K_{E i l}+b_{l}}}\right\}-1\right)\left(\exp \left\{-\theta \mathrm{e}^{-H_{2}\left(y_{G i l} \mid \boldsymbol{\lambda}_{2}\right) \mathrm{e}^{K_{G i l}+b_{l}}}\right\}-1\right)\right]^{2}}\right]^{\mathbb{I}\left\{\begin{array}{c}
d_{i l}=1 \\
v_{i l}=1
\end{array}\right\}}} \\
& \times\left[( \operatorname { e x p } \{ - \theta \mathrm { e } ^ { - H _ { 2 } ( y _ { G i l } | \boldsymbol { \lambda } _ { 2 } ) \mathrm { e } ^ { K _ { G i l } + b _ { l } } } \} - 1 ) \left[\mathrm{e}^{-\theta}+\exp \left\{-\theta\left(\mathrm{e}^{-H_{1}\left(y_{E i l} \mid \boldsymbol{\lambda}_{1}\right) \mathrm{e}^{K_{E i l}+b_{l}}}\right.\right.\right.\right. \\
& \left.\left.+\mathrm{e}^{-H_{2}\left(y_{G i l} \mid \boldsymbol{\lambda}_{2}\right) \mathrm{e}^{K_{G i l}+b_{l}}}\right)\right\}-\exp \left\{-\theta \mathrm{e}^{-H_{1}\left(y_{E i l} \mid \boldsymbol{\lambda}_{1}\right) \mathrm{e}^{K_{E i l}+b_{l}}}\right\} \\
& \left.\left.-\exp \left\{-\theta \mathrm{e}^{-H_{2}\left(y_{G i l} \mid \boldsymbol{\lambda}_{2}\right) \mathrm{e}^{K_{G i l}+b_{l}}}\right\}\right]^{-1}\right]^{\mathbb{I}\left\{d_{i l l}^{d_{i l}=0}\right\}} \exp \left\{-0,5\left(\boldsymbol{\beta}_{2}^{\top}, \boldsymbol{\gamma}_{2}^{\top}\right)^{\top} \boldsymbol{\Sigma}_{2}^{-1}\left(\boldsymbol{\beta}_{2}^{\top}, \boldsymbol{\gamma}_{2}^{\top}\right)\right\},
\end{aligned}
$$

com a função log-posteriori dada por

$$
\begin{aligned}
& \mathcal{L}\left(\pi\left(\boldsymbol{\beta}_{2}, \boldsymbol{\gamma}_{2} \mid \beta_{1}, \boldsymbol{\gamma}_{1}, \boldsymbol{\lambda}_{1}, \boldsymbol{\lambda}_{2}, \boldsymbol{b}, \theta, D_{o b s}\right)\right)=\sum_{l=1}^{L} \sum_{i=1}^{n_{l}} \mathbb{I}\left\{\begin{array}{l}
d_{i l}=1 \\
v_{i l}=1
\end{array}\right\} \\
& \times\left(K_{G i l}-H_{2}\left(y_{G i l} \mid \boldsymbol{\lambda}_{2}\right) \mathrm{e}^{K_{G i l}+b_{l}}-\theta \mathrm{e}^{-H_{2}\left(y_{G i l} \mid \boldsymbol{\lambda}_{2}\right) \mathrm{e}^{K_{G i l}+b_{l}}}-2 \ln \left(\mathrm{e}^{-\theta}-1\right.\right. \\
& \left.\left.+\left(\exp \left\{-\theta \mathrm{e}^{-H_{1}\left(y_{E i l} \mid \boldsymbol{\lambda}_{1}\right) \mathrm{e}^{K_{E i l}+b_{l}}}\right\}-1\right)\left(\exp \left\{-\theta \mathrm{e}^{-H_{2}\left(y_{G i l} \mid \boldsymbol{\lambda}_{2}\right) \mathrm{e}^{K_{G i l}+b_{l}}}\right\}-1\right)\right)\right) \\
& +\sum_{l=1}^{L} \sum_{i=1}^{n_{l}} \mathbb{I}\left\{\begin{array}{l}
d_{i l}=1 \\
v_{i l}=0
\end{array}\right\}\left(\ln \left(\exp \left\{-\theta \mathrm{e}^{-H_{2}\left(y_{G i l} \mid \boldsymbol{\lambda}_{2}\right) \mathrm{e}^{K_{G i l}+b_{l}}}\right\}-1\right)-\ln \left(\mathrm{e}^{-\theta}\right.\right. \\
& +\exp \left\{-\theta\left(\mathrm{e}^{-H_{1}\left(y_{E i l} \mid \boldsymbol{\lambda}_{1}\right) \mathrm{e}^{K_{E i l}+b_{l}}}+\mathrm{e}^{-H_{2}\left(y_{G i l} \mid \boldsymbol{\lambda}_{2}\right) \mathrm{e}^{K_{G i l}+b_{l}}}\right)\right\}-\exp \left\{-\theta \mathrm{e}^{-H_{1}\left(y_{E i l} \mid \boldsymbol{\lambda}_{1}\right) \mathrm{e}^{K_{E i l}+b_{l}}}\right\} \\
& \left.\left.-\exp \left\{-\theta \mathrm{e}^{-H_{2}\left(y_{G i l} \mid \boldsymbol{\lambda}_{2}\right) \mathrm{e}^{K_{G i l}+b_{l}}}\right\}\right)\right)-0,5\left(\boldsymbol{\beta}_{2}^{\top}, \boldsymbol{\gamma}_{2}^{\top}\right)^{\top} \boldsymbol{\Sigma}_{2}^{-1}\left(\boldsymbol{\beta}_{2}^{\top}, \boldsymbol{\gamma}_{2}^{\top}\right) \text {. }
\end{aligned}
$$

7. Distribuição a posteriori condicional completa da variável latente $\boldsymbol{E}^{*}$

Esta distribuição se conserva como na cópula de Clayton, e portanto aqui é omitida, veja (3.21).

8. Distribuição a posteriori condicional completa do parâmetro da cópula $\theta$ De (3.27) e pela independência entre parâmetros tem-se que a distribuição a posteriori condicional completa para $\theta$ fica expressa como 


$$
\begin{aligned}
& \pi\left(\theta \mid \beta_{1}, \boldsymbol{\gamma}_{1}, \boldsymbol{\lambda}_{1}, \boldsymbol{\beta}_{2}, \boldsymbol{\gamma}_{2}, \boldsymbol{\lambda}_{2}, \boldsymbol{b}, \boldsymbol{E}^{*}, D_{o b s}\right) \propto \prod_{l=1}^{L} \prod_{i=1}^{n_{l}}
\end{aligned}
$$

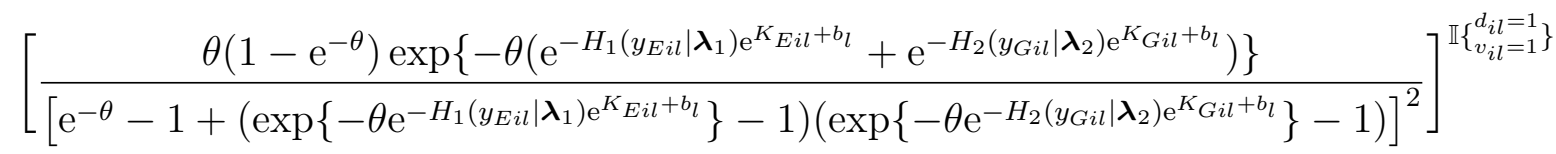

$$
\begin{aligned}
& \times\left[\exp \left\{-\theta \mathrm{e}^{-H_{1}\left(y_{E i l} \mid \boldsymbol{\lambda}_{1}\right) \mathrm{e}^{K_{E i l}+b_{l}}}\right\}\left(\exp \left\{-\theta \mathrm{e}^{-H_{2}\left(y_{G i l} \mid \boldsymbol{\lambda}_{2}\right) \mathrm{e}^{K_{G i l}+b_{l}}}\right\}-1\right)\right. \\
& \times\left[\mathrm{e}^{-\theta}+\exp \left\{-\theta\left(\mathrm{e}^{-H_{1}\left(y_{E i l} \mid \boldsymbol{\lambda}_{1}\right) \mathrm{e}^{K_{E i l}+b_{l}}}+\mathrm{e}^{-H_{2}\left(y_{G i l} \mid \boldsymbol{\lambda}_{2}\right) \mathrm{e}^{K_{G i l}+b_{l}}}\right)\right\}\right. \\
& \left.\left.-\exp \left\{-\theta \mathrm{e}^{-H_{1}\left(y_{E i l} \mid \boldsymbol{\lambda}_{1}\right) \mathrm{e}^{K_{E i l}+b_{l}}}\right\}-\exp \left\{-\theta \mathrm{e}^{-H_{2}\left(y_{G i l} \mid \boldsymbol{\lambda}_{2}\right) \mathrm{e}^{K_{G i l}+b_{l}}}\right\}\right]^{-1}\right]^{\mathbb{I}\left\{\begin{array}{c}
d_{i l}=1 \\
v_{i l}=0
\end{array}\right\}} \exp \left\{-\frac{\theta^{2}}{2 \sigma_{\theta}^{2}}\right\},
\end{aligned}
$$

e a função log-posteriori por

$$
\begin{aligned}
\mathcal{L} & \left.\pi\left(\theta \mid \beta_{1}, \boldsymbol{\gamma}_{1}, \boldsymbol{\lambda}_{1}, \boldsymbol{\beta}_{2}, \boldsymbol{\gamma}_{2}, \boldsymbol{\lambda}_{2}, \boldsymbol{b}, \boldsymbol{E}^{*}, D_{o b s}\right)\right)=\sum_{l=1}^{L} \sum_{i=1}^{n_{l}} \mathbb{I}\left\{\begin{array}{l}
d_{i l}=1 \\
v_{i l}=1
\end{array}\right\} \\
& \times\left(\ln \left(\theta\left(1-\mathrm{e}^{-\theta}\right)\right)-\theta\left(\mathrm{e}^{-H_{1}\left(y_{E i l} \mid \boldsymbol{\lambda}_{1}\right) \mathrm{e}^{K_{E i l}+b_{l}}}+\mathrm{e}^{-H_{2}\left(y_{G i l} \mid \boldsymbol{\lambda}_{2}\right) \mathrm{e}^{K_{G i l}+b_{l}}}\right)\right. \\
& \left.-2 \ln \left(\mathrm{e}^{-\theta}-1+\left(\exp \left\{-\theta \mathrm{e}^{-H_{1}\left(y_{E i l} \mid \boldsymbol{\lambda}_{1}\right) \mathrm{e}^{K_{E i l}+b_{l}}}\right\}-1\right)\left(\exp \left\{-\theta \mathrm{e}^{-H_{2}\left(y_{G i l} \mid \boldsymbol{\lambda}_{2}\right) \mathrm{e}^{K_{G i l}+b_{l}}}\right\}-1\right)\right)\right) \\
& -\sum_{l=1}^{L} \sum_{i=1}^{n_{l}} \mathbb{I}\left\{\begin{array}{l}
d_{i l}=1 \\
v_{i l}=0
\end{array}\right\}\left(\theta \mathrm{e}^{-H_{1}\left(y_{E i l} \mid \boldsymbol{\lambda}_{1}\right) \mathrm{e}^{K_{E i l}+b_{l}}}+\ln \left(\exp \left\{-\theta \mathrm{e}^{-H_{2}\left(y_{G i l} \mid \boldsymbol{\lambda}_{2}\right) \mathrm{e}^{K_{G i l}+b_{l}}}\right\}-1\right)\right. \\
& -\ln \left(\mathrm{e}^{-\theta}+\exp \left\{-\theta\left(\mathrm{e}^{-H_{1}\left(y_{E i l} \mid \boldsymbol{\lambda}_{1}\right) \mathrm{e}^{K_{E i l}+b_{l}}}+\mathrm{e}^{-H_{2}\left(y_{G i l} \mid \boldsymbol{\lambda}_{2}\right) \mathrm{e}^{K_{G i l}+b_{l}}}\right)\right\}\right. \\
& \left.\left.-\exp \left\{-\theta \mathrm{e}^{-H_{1}\left(y_{E i l} \mid \boldsymbol{\lambda}_{1}\right) \mathrm{e}^{K_{E i l}+b_{l}}}\right\}-\exp \left\{-\theta \mathrm{e}^{-H_{2}\left(y_{G i l} \mid \boldsymbol{\lambda}_{2}\right) \mathrm{e}^{K_{G i l}+b_{l}}}\right\}\right)\right)-\frac{\theta^{2}}{2 \sigma_{\theta}^{2}} .
\end{aligned}
$$

9. Distribuição a posteriori condicional completa dos coeficientes do modelo logito $\alpha$

Esta distribuição também se conserva como na cópula de Clayton, veja (3.22).

10. Distribuição a posteriori condicional completa da variância do efeito aleatório $B$.

Como na cópula de Clayton $[B \mid \boldsymbol{b}] \sim \operatorname{Gama} \operatorname{Inversa}\left(\frac{L}{2}+\kappa_{B} ; \frac{\sum_{l=1}^{L} b_{l}^{2}}{2}+\varsigma_{B}\right)$.

11. Distribuição a posteriori condicional completa do vetor de efeitos aleatórios $\boldsymbol{b}$

Examinando a distribuição a posteriori conjunta aumentada (3.27), obtém-se 


$$
\begin{aligned}
& \pi\left(\boldsymbol{b} \mid \beta_{0}, \boldsymbol{\gamma}_{0}, \beta_{1}, \boldsymbol{\gamma}_{1}, \boldsymbol{\beta}_{2}, \boldsymbol{\gamma}_{2}, \boldsymbol{\lambda}_{0}, \boldsymbol{\lambda}_{1}, \boldsymbol{\lambda}_{2}, \theta, B, D_{o b s}\right] \propto \prod_{l=1}^{L} \prod_{i=1}^{n_{l}}\left[\operatorname { e x p } \left\{b_{l}\right.\right. \\
& \left.\left.-H_{0}\left(y_{i l} \mid \boldsymbol{\lambda}_{0}\right) \mathrm{e}^{K_{D i l}+b_{l}}\right\}\right]^{\mathbb{I}\left\{\begin{array}{c}
d_{i l}=0 \\
v_{i l}=1
\end{array}\right\}}\left[\exp \left\{2 b_{l}-H_{1}\left(y_{E i l} \mid \boldsymbol{\lambda}_{1}\right) \mathrm{e}^{K_{E i l}+b_{l}}-H_{2}\left(y_{G i l} \mid \boldsymbol{\lambda}_{2}\right) \mathrm{e}^{K_{G i l}+b_{l}}\right\}\right. \\
& \left.\times \frac{\exp \left\{-\theta\left(\mathrm{e}^{-H_{1}\left(y_{E i l} \mid \boldsymbol{\lambda}_{1}\right) \mathrm{e}^{K_{E i l}+b_{l}}}+\mathrm{e}^{-H_{2}\left(y_{G i l} \mid \boldsymbol{\lambda}_{2}\right) \mathrm{e}^{K_{G i l}+b_{l}}}\right)\right\}}{\left[\mathrm{e}^{-\theta}-1+\left(\exp \left\{-\theta \mathrm{e}^{-H_{1}\left(y_{E i l} \mid \boldsymbol{\lambda}_{1}\right) \mathrm{e}^{K_{E i l}+b_{l}}}\right\}-1\right)\left(\exp \left\{-\theta \mathrm{e}^{-H_{2}\left(y_{G i l} \mid \boldsymbol{\lambda}_{2}\right) \mathrm{e}^{K_{G i l}+b_{l}}}\right\}-1\right)\right]^{2}}\right]^{\mathbb{I}\left\{\begin{array}{c}
d_{i l}=1 \\
d_{i l}=1
\end{array}\right\}} \\
& \times\left[\operatorname { e x p } \{ b _ { l } - H _ { 1 } ( y _ { E i l } | \boldsymbol { \lambda } _ { 1 } ) \mathrm { e } ^ { K _ { E i l } + b _ { l } } - \theta \mathrm { e } ^ { - H _ { 1 } ( y _ { E i l } | \boldsymbol { \lambda } _ { 1 } ) \mathrm { e } ^ { K _ { E i l } + b _ { l } } } \} \left(\exp \left\{-\theta \mathrm{e}^{-H_{2}\left(y_{G i l} \mid \boldsymbol{\lambda}_{2}\right) \mathrm{e}^{K_{G i l}+b_{l}}}\right\}\right.\right. \\
& -1)\left[\mathrm{e}^{-\theta}+\exp \left\{-\theta\left(\mathrm{e}^{-H_{1}\left(y_{E i l} \mid \boldsymbol{\lambda}_{1}\right) \mathrm{e}^{K_{E i l}+b_{l}}}+\mathrm{e}^{-H_{2}\left(y_{G i l} \mid \boldsymbol{\lambda}_{2}\right) \mathrm{e}^{K_{G i l}+b_{l}}}\right)\right\}\right. \\
& \left.\left.-\exp \left\{-\theta \mathrm{e}^{-H_{1}\left(y_{E i l} \mid \boldsymbol{\lambda}_{1}\right) \mathrm{e}^{K_{E i l}+b_{l}}}\right\}-\exp \left\{-\theta \mathrm{e}^{-H_{2}\left(y_{G i l} \mid \boldsymbol{\lambda}_{2}\right) \mathrm{e}^{K_{G i l}+b_{l}}}\right\}\right]^{-1}\right]^{\mathbb{I}\left\{\begin{array}{l}
d_{i l}=1 \\
v_{i l}=0
\end{array}\right.}\left[\operatorname { e x p } \left\{-E_{i l}^{*}\right.\right. \\
& \left.\left.\times H_{1}\left(y_{i l} \mid \boldsymbol{\lambda}_{1}\right) \mathrm{e}^{K_{E i l}+b_{l}}\right\} \exp \left\{-\left(1-E_{i l}^{*}\right) H_{0}\left(y_{i l} \mid \boldsymbol{\lambda}_{0}\right) \mathrm{e}^{K_{D i l}+b_{l}}\right\}\right]^{\mathbb{I}\left\{v_{i l}^{d_{i l}=0}\right\}} \exp \left\{-\frac{b_{l}^{2}}{2 B}\right\},
\end{aligned}
$$

com a função log-posteriori expressa como

$$
\begin{aligned}
& \mathcal{L}\left(\pi\left(\boldsymbol{b} \mid \beta_{0}, \boldsymbol{\gamma}_{0}, \beta_{1}, \boldsymbol{\gamma}_{1}, \boldsymbol{\beta}_{2}, \boldsymbol{\gamma}_{2}, \boldsymbol{\lambda}_{0}, \boldsymbol{\lambda}_{1}, \boldsymbol{\lambda}_{2}, \theta, B, D_{\text {obs }}\right)\right)=\sum_{l=1}^{L} \sum_{i=1}^{n_{l}} \mathbb{I}\left\{\begin{array}{l}
d_{i l}=0 \\
v_{i l}=1
\end{array}\right\}\left(b_{l}\right. \\
& \left.-H_{0}\left(y_{i l} \mid \boldsymbol{\lambda}_{0}\right) \mathrm{e}^{K_{D i l}+b_{l}}\right)+\sum_{l=1}^{L} \sum_{i=1}^{n_{l}} \mathbb{I}\left\{\begin{array}{l}
d_{i l}=1 \\
v_{i l}=1
\end{array}\right\}\left(2 b_{l}-H_{1}\left(y_{E i l} \mid \boldsymbol{\lambda}_{1}\right) \mathrm{e}^{K_{E i l}+b_{l}}\right. \\
& -H_{2}\left(y_{G i l} \mid \boldsymbol{\lambda}_{2}\right) \mathrm{e}^{K_{G i l}+b_{l}}-\theta\left(\mathrm{e}^{-H_{1}\left(y_{E i l} \mid \boldsymbol{\lambda}_{1}\right) \mathrm{e}^{K_{E i l}+b_{l}}}+\mathrm{e}^{-H_{2}\left(y_{G i l} \mid \boldsymbol{\lambda}_{2}\right) \mathrm{e}^{K_{G i l}+b_{l}}}\right) \\
& \left.-2 \ln \left(\mathrm{e}^{-\theta}-1+\left(\exp \left\{-\theta \mathrm{e}^{-H_{1}\left(y_{E i l} \mid \boldsymbol{\lambda}_{1}\right) \mathrm{e}^{K_{E i l}+b_{l}}}\right\}-1\right)\left(\exp \left\{-\theta \mathrm{e}^{-H_{2}\left(y_{G i l} \mid \boldsymbol{\lambda}_{2}\right) \mathrm{e}^{K_{G i l}+b_{l}}}\right\}-1\right)\right)\right) \\
& +\sum_{l=1}^{L} \sum_{i=1}^{n_{l}} \mathbb{I}\left\{\begin{array}{c}
d_{i l}=1 \\
v_{i l}=0
\end{array}\right\}\left(b_{l}-H_{1}\left(y_{E i l} \mid \boldsymbol{\lambda}_{1}\right) \mathrm{e}^{K_{E i l}+b_{l}}-\theta \mathrm{e}^{-H_{1}\left(y_{E i l} \mid \boldsymbol{\lambda}_{1}\right) \mathrm{e}^{K_{E i l}+b_{l}}}+\ln (\exp \{-\theta\right. \\
& \left.\left.\times \mathrm{e}^{-H_{2}\left(y_{G i l} \mid \boldsymbol{\lambda}_{2}\right) \mathrm{e}^{K_{G i l}+b_{l}}}\right\}-1\right)-\ln \left(\mathrm{e}^{-\theta}+\exp \left\{-\theta\left(\mathrm{e}^{-H_{1}\left(y_{E i l} \mid \boldsymbol{\lambda}_{1}\right) \mathrm{e}^{K_{E i l}+b_{l}}}\right.\right.\right. \\
& \left.\left.\left.\left.+\mathrm{e}^{-H_{2}\left(y_{G i l} \mid \boldsymbol{\lambda}_{2}\right) \mathrm{e}^{K_{G i l}+b_{l}}}\right)\right\}-\exp \left\{-\theta \mathrm{e}^{-H_{1}\left(y_{E i l} \mid \boldsymbol{\lambda}_{1}\right) \mathrm{e}^{K_{E i l}+b_{l}}}\right\}-\exp \left\{-\theta \mathrm{e}^{-H_{2}\left(y_{G i l} \mid \boldsymbol{\lambda}_{2}\right) \mathrm{e}^{K_{G i l}+b_{l}}}\right\}\right)\right) \\
& -\sum_{l=1}^{L} \sum_{i=1}^{n_{l}} \mathbb{I}\left\{\begin{array}{c}
d_{i l}=0 \\
v_{i l}=0
\end{array}\right\}\left(E_{i l}^{*} H_{1}\left(y_{i l} \mid \boldsymbol{\lambda}_{1}\right) \mathrm{e}^{K_{E i l}+b_{l}}+\left(1-E_{i l}^{*}\right) H_{0}\left(y_{i l} \mid \boldsymbol{\lambda}_{0}\right) \mathrm{e}^{K_{D i l}+b_{l}}\right)-\sum_{l=1}^{L} \frac{b_{l}^{2}}{2 B} \text {. }
\end{aligned}
$$

\subsection{Cópula de Gumbel}

Considerando a definição da cópula de Gumbel ou fragilidade estável positiva como descrito na Seção 2.2.4, tem-se

$$
\mathcal{C}_{\theta}(u, v)=\exp \left\{-\left[(-\ln u)^{\theta}+(-\ln v)^{\theta}\right]^{\frac{1}{\theta}}\right\}, \quad \theta \in[1, \infty) .
$$


Assumindo $u$ e $v$ como as funções de sobrevivência marginais, ou seja,

$$
\begin{gathered}
u=S_{E}\left(t_{E} \mid A, \boldsymbol{x}, E=1, b\right)=\exp \left\{-H_{1}\left(t_{E}\right) \exp \left\{K_{E}+b\right\}\right\} \mathrm{e} \\
v=S_{G}\left(t_{G} \mid A, V, \boldsymbol{z}, E=1, b\right)=\exp \left\{-H_{2}\left(t_{G}\right) \exp \left\{K_{G}+b\right\}\right\},
\end{gathered}
$$

obtem-se a função de sobrevivência conjunta dos tempos $T_{E}$ e $T_{G}$ para a componente do modelo (3.3) sendo expressa por

$$
\begin{aligned}
S_{E G_{\theta}}\left(t_{E}, t_{G} \mid A, \boldsymbol{x}, b\right) & =\exp \left\{-\left[\left(-\ln \exp \left\{-H_{1}\left(t_{E}\right) \mathrm{e}^{K_{E}+b}\right\}\right)^{\theta}+\left(-\ln \exp \left\{-H_{2}\left(t_{G}\right) \mathrm{e}^{K_{G}+b}\right\}\right)^{\theta}\right]^{\frac{1}{\theta}}\right\} \\
& =\exp \left\{-\left[\left(H_{1}\left(t_{E}\right) \mathrm{e}^{K_{E}+b}\right)^{\theta}+\left(H_{2}\left(t_{G}\right) \mathrm{e}^{K_{G}+b}\right)^{\theta}\right]^{\frac{1}{\theta}}\right\}
\end{aligned}
$$

com função de densidade bivariada dada por

$$
\begin{aligned}
& \quad f_{E G_{\theta}}\left(t_{E}, t_{G} \mid A, V, \boldsymbol{x}, \boldsymbol{z}, b\right)=h_{1}\left(t_{E}\right) h_{2}\left(t_{G}\right)\left(H_{1}\left(t_{E}\right) H_{2}\left(t_{G}\right)\right)^{\theta-1} \\
& \quad \times \exp \left\{\theta\left(K_{E}+K_{G}+2 b\right)-\left[\left(H_{1}\left(t_{E}\right) \mathrm{e}^{K_{E}+b}\right)^{\theta}+\left(H_{2}\left(t_{G}\right) \mathrm{e}^{K_{G}+b}\right)^{\theta}\right]^{\frac{1}{\theta}}\right\} \\
& \times\left[\left(H_{1}\left(t_{E}\right) \mathrm{e}^{K_{E}+b}\right)^{\theta}+\left(H_{2}\left(t_{G}\right) \mathrm{e}^{K_{G}+b}\right)^{\theta}\right]^{\frac{1}{\theta}-2}\left(\theta-1+\left[\left(H_{1}\left(t_{E}\right) \mathrm{e}^{K_{E}+b}\right)^{\theta}+\left(H_{2}\left(t_{G}\right) \mathrm{e}^{K_{G}+b}\right)^{\theta}\right]^{\frac{1}{\theta}}\right) .
\end{aligned}
$$

A partir das funções (3.28) e (3.29), obtem-se as expressões de cada caso para a construção da função de verossimilhança (3.11) segundo os dados observados do indivíduo $i$ no grupo $l$, assim:

$$
\begin{aligned}
& \text { - } L_{1 i l}\left(\boldsymbol{\alpha}, \beta_{0}, \boldsymbol{\gamma}_{0}, \boldsymbol{\lambda}_{0} \mid D_{i l}, b_{l}\right)=\frac{1}{1+\mathrm{e}^{K_{P i l}}} h_{0}\left(y_{i l} \mid \boldsymbol{\lambda}_{0}\right) \mathrm{e}^{K_{D i l}+b_{l}} \exp \left\{-H_{0}\left(y_{i l} \mid \boldsymbol{\lambda}_{0}\right) \mathrm{e}^{K_{D i l}+b_{l}}\right\} ; \\
& \text { - } L_{2 i l}\left(\boldsymbol{\alpha}, \beta_{1}, \boldsymbol{\gamma}_{1}, \boldsymbol{\lambda}_{1}, \boldsymbol{\beta}_{2}, \boldsymbol{\gamma}_{2}, \boldsymbol{\lambda}_{2}, \theta \mid D_{i l}, h_{l}\right)=\frac{\mathrm{e}^{K_{P i l}}}{1+\mathrm{e}^{K_{P i l}}} h_{1}\left(y_{E i l} \mid \boldsymbol{\lambda}_{1}\right) h_{2}\left(y_{G i l} \mid \boldsymbol{\lambda}_{2}\right) \\
& \times\left(H_{1}\left(y_{E i l} \mid \boldsymbol{\lambda}_{1}\right) H_{2}\left(y_{G i l} \mid \boldsymbol{\lambda}_{2}\right)\right)^{\theta-1} \exp \left\{\theta\left(K_{E i l}+K_{G i l}+2 b_{l}\right)\right. \\
& \left.-\left[\left(H_{1}\left(y_{E i l} \mid \boldsymbol{\lambda}_{1}\right) \mathrm{e}^{K_{E i l}+b_{l}}\right)^{\theta}+\left(H_{2}\left(y_{G i l} \mid \boldsymbol{\lambda}_{2}\right) \mathrm{e}^{K_{G i l}+b_{l}}\right)^{\theta}\right]^{\frac{1}{\theta}}\right\} \\
& \times\left[\left(H_{1}\left(y_{E i l} \mid \boldsymbol{\lambda}_{1}\right) \mathrm{e}^{K_{E i l}+b_{l}}\right)^{\theta}+\left(H_{2}\left(y_{G i l} \mid \boldsymbol{\lambda}_{2}\right) \mathrm{e}^{K_{G i l}+b_{l}}\right)^{\theta}\right]^{\frac{1}{\theta}-2} \\
& \times\left(\theta-1+\left[\left(H_{1}\left(y_{E i l} \mid \boldsymbol{\lambda}_{1}\right) \mathrm{e}^{K_{E i l}+b_{l}}\right)^{\theta}+\left(H_{2}\left(y_{G i l} \mid \boldsymbol{\lambda}_{2}\right) \mathrm{e}^{K_{G i l}+b_{l}}\right)^{\theta}\right]^{\frac{1}{\theta}}\right) ; \\
& \text { - } L_{3 i l}\left(\boldsymbol{\alpha}, \beta_{1}, \boldsymbol{\gamma}_{1}, \boldsymbol{\lambda}_{1}, \boldsymbol{\beta}_{2}, \boldsymbol{\gamma}_{2}, \boldsymbol{\lambda}_{2}, \theta \mid D_{i l}, b_{l}\right)=\frac{\mathrm{e}^{K_{P i l}}}{1+\mathrm{e}^{K_{P i l}}} h_{1}\left(y_{E i l} \mid \boldsymbol{\lambda}_{1}\right) \exp \left\{K_{E i l}+b_{l}\right. \\
& \left.-H_{1}\left(y_{E i l} \mid \boldsymbol{\lambda}_{1}\right) \mathrm{e}^{K_{E i l}+b_{l}}-\left[\left(H_{1}\left(y_{E i l} \mid \boldsymbol{\lambda}_{1}\right) \mathrm{e}^{K_{E i l}+b_{l}}\right)^{\theta}+\left(H_{2}\left(y_{G i l} \mid \boldsymbol{\lambda}_{2}\right) \mathrm{e}^{K_{G i l}+b_{l}}\right)^{\theta}\right]^{\frac{1}{\theta}}\right\} \\
& \times\left[\left(H_{1}\left(y_{E i l} \mid \boldsymbol{\lambda}_{1}\right) \mathrm{e}^{K_{E i l}+b_{l}}\right)^{\theta}+\left(H_{2}\left(y_{G i l} \mid \boldsymbol{\lambda}_{2}\right) \mathrm{e}^{K_{G i l}+b_{l}}\right)^{\theta}\right]^{\frac{1}{\theta}-1} ;
\end{aligned}
$$




$$
\begin{aligned}
& -L_{4 i}\left(\boldsymbol{\alpha}, \beta_{0}, \boldsymbol{\gamma}_{0}, \boldsymbol{\lambda}_{0}, \beta_{1}, \boldsymbol{\gamma}_{1}, \boldsymbol{\lambda}_{1}, \theta \mid D_{i l}, b_{l}\right)= \\
& \quad \frac{\mathrm{e}^{K_{P} i l}}{1+\mathrm{e}^{K_{P} i l}} \exp \left\{-H_{1}\left(y_{i l} \mid \boldsymbol{\lambda}_{1}\right) \mathrm{e}^{K_{E i l}+b_{l}}\right\}+\frac{1}{1+\mathrm{e}^{K_{P} i l}} \exp \left\{-H_{0}\left(y_{i l} \mid \boldsymbol{\lambda}_{0}\right) \mathrm{e}^{K_{D i l}+b_{l}}\right\} .
\end{aligned}
$$

Analogamente como nas cópulas anteriores e, considerando uma distribuição normal truncada no intervalo $[1, \infty)$ como distribuição a priori para o parâmetro da cópula, a distribuição a posteriori completa aumentada (3.14) segundo a cópula de Gumbel é dada por

$$
\begin{aligned}
& \pi\left(\boldsymbol{\xi}, B, \boldsymbol{b}, \boldsymbol{E}^{*} \mid D_{o b s}\right) \propto\left(\prod _ { l = 1 } ^ { L } \prod _ { i = 1 } ^ { n _ { l } } \left[\frac { 1 } { 1 + \mathrm { e } ^ { K _ { P i l } } } h _ { 0 } ( y _ { i l } | \boldsymbol { \lambda } _ { 0 } ) \mathrm { e } ^ { K _ { D i l } + b _ { l } } \operatorname { e x p } \left\{-H_{0}\left(y_{i l} \mid \boldsymbol{\lambda}_{0}\right)\right.\right.\right. \\
& \left.\left.\times \mathrm{e}^{K_{D i l}+b_{l}}\right\}\right]^{\mathbb{I}\left\{\begin{array}{c}
d_{i l}=0 \\
v_{i l}=1
\end{array}\right.}\left[\frac{\mathrm{e}^{K_{P i l}}}{1+\mathrm{e}^{K_{P i l}}} h_{1}\left(y_{E i l} \mid \boldsymbol{\lambda}_{1}\right) h_{2}\left(y_{G i l} \mid \boldsymbol{\lambda}_{2}\right)\left(H_{1}\left(y_{E i l} \mid \boldsymbol{\lambda}_{1}\right) H_{2}\left(y_{G i l} \mid \boldsymbol{\lambda}_{2}\right)\right)^{\theta-1}\right. \\
& \times \exp \left\{\theta\left(K_{E i l}+K_{G i l}+2 b_{l}\right)-\left[\left(H_{1}\left(y_{E i l} \mid \boldsymbol{\lambda}_{1}\right) \mathrm{e}^{K_{E i l}+b_{l}}\right)^{\theta}+\left(H_{2}\left(y_{G i l} \mid \boldsymbol{\lambda}_{2}\right) \mathrm{e}^{K_{G i l}+b_{l}}\right)^{\theta}\right]^{\frac{1}{\theta}}\right\} \\
& \times\left[\left(H_{1}\left(y_{E i l} \mid \boldsymbol{\lambda}_{1}\right) \mathrm{e}^{K_{E i l}+b_{l}}\right)^{\theta}+\left(H_{2}\left(y_{G i l} \mid \boldsymbol{\lambda}_{2}\right) \mathrm{e}^{K_{G i l}+b_{l}}\right)^{\theta}\right]^{\frac{1}{\theta}-2}\left(\theta-1+\left[\left(H_{1}\left(y_{E i l} \mid \boldsymbol{\lambda}_{1}\right) \mathrm{e}^{K_{E i l}+b_{l}}\right)^{\theta}\right.\right. \\
& \left.\left.\left.+\left(H_{2}\left(y_{G i l} \mid \boldsymbol{\lambda}_{2}\right) \mathrm{e}^{K_{G i l}+b_{l}}\right)^{\theta}\right]^{\frac{1}{\theta}}\right)\right]^{\mathbb{I}\left\{d_{i l l}^{d_{i l}=1}\right\}}\left[\frac { \mathrm { e } ^ { K _ { P i l } } } { 1 + \mathrm { e } ^ { K _ { P i l } } } h _ { 1 } ( y _ { E i l } | \boldsymbol { \lambda } _ { 1 } ) \operatorname { e x p } \left\{K_{E i l}+b_{l}\right.\right. \\
& \left.-H_{1}\left(y_{E i l} \mid \boldsymbol{\lambda}_{1}\right) \mathrm{e}^{K_{E i l}+b_{l}}-\left[\left(H_{1}\left(y_{E i l} \mid \boldsymbol{\lambda}_{1}\right) \mathrm{e}^{K_{E i l}+b_{l}}\right)^{\theta}+\left(H_{2}\left(y_{G i l} \mid \boldsymbol{\lambda}_{2}\right) \mathrm{e}^{K_{G i l}+b_{l}}\right)^{\theta}\right]^{\frac{1}{\theta}}\right\} \\
& \left.\times\left[\left(H_{1}\left(y_{E i l} \mid \boldsymbol{\lambda}_{1}\right) \mathrm{e}^{K_{E i l}+b_{l}}\right)^{\theta}+\left(H_{2}\left(y_{G i l} \mid \boldsymbol{\lambda}_{2}\right) \mathrm{e}^{K_{G i l}+b_{l}}\right)^{\theta}\right]^{\frac{1}{\theta}-1}\right]^{\mathbb{I}\left\{\begin{array}{c}
d_{i l}=1 \\
v_{i l}=0
\end{array}\right\}}\left[\left(\frac{\mathrm{e}^{K_{P} i l}}{1+\mathrm{e}^{K_{P} i l}}\right.\right. \\
& \left.\left.\times \exp \left\{-H_{1}\left(y_{i l} \mid \boldsymbol{\lambda}_{1}\right) \mathrm{e}^{K_{E i l}+b_{l}}\right\}\right)^{E_{i l}^{*}}\left(\frac{1}{1+\mathrm{e}^{K_{P} i l}} \exp \left\{-H_{0}\left(y_{i l} \mid \boldsymbol{\lambda}_{0}\right) \mathrm{e}^{K_{D i l}+b_{l}}\right\}\right)^{1-E_{i l}^{*}}\right]^{\mathbb{I}\left\{\begin{array}{c}
d_{i l}=0 \\
v_{i l}=0
\end{array}\right\}} \\
& \left.(2 \pi B)^{-\frac{1}{2}} \exp \left\{-\frac{b_{l}^{2}}{2 B}\right\}\right)\left(\exp \left\{-0,5\left(\alpha_{0}, \alpha_{1}, \boldsymbol{\alpha}_{2}^{\top}\right)^{\top} \boldsymbol{\Sigma}_{\alpha}^{-1}\left(\alpha_{0}, \alpha_{1}, \boldsymbol{\alpha}_{2}^{\top}\right)\right\}\right. \\
& \times \exp \left\{-0,5\left(\beta_{0}, \boldsymbol{\gamma}_{0}^{\top}\right)^{\top} \boldsymbol{\Sigma}_{0}^{-1}\left(\beta_{0}, \boldsymbol{\gamma}_{0}^{\top}\right)\right\} \exp \left\{-0,5\left(\beta_{1}, \boldsymbol{\gamma}_{1}^{\top}\right)^{\top} \boldsymbol{\Sigma}_{1}^{-1}\left(\beta_{1}, \boldsymbol{\gamma}_{1}^{\top}\right)\right\} \\
& \times \exp \left\{-0,5\left(\boldsymbol{\beta}_{2}^{\top}, \boldsymbol{\gamma}_{2}^{\top}\right)^{\top} \boldsymbol{\Sigma}_{2}^{-1}\left(\boldsymbol{\beta}_{2}^{\top}, \boldsymbol{\gamma}_{2}^{\top}\right)\right\} \prod_{j=1}^{J 0} \lambda_{0 j}^{a_{0 j}-1} \exp \left\{-b_{0 j} \lambda_{0 j}\right\} \prod_{j=1}^{J 1} \lambda_{1 j}^{a_{1 j}-1} \exp \left\{-b_{1 j} \lambda_{1 j}\right\} \\
& \left.\times \prod_{j=1}^{J 2} \lambda_{2 j}^{a_{2 j}-1} \exp \left\{-b_{2 j} \lambda_{2 j}\right\} \exp \left\{-\frac{\theta^{2}}{2 \sigma_{\theta}^{2}}\right\}\left(\frac{1}{B}\right)^{\kappa_{B}+1} \exp \left\{-\frac{\varsigma_{B}}{B}\right\}\right) .
\end{aligned}
$$

A partir desta distribuição a posteriori são obtidas as distribuições condicionais de cada parâmetro.

1. Distribuição a posteriori condicional completa de $\boldsymbol{\lambda}_{0}$

Corresponde com a mesma distribuição descrita para a cópula de Clayton, dada pela expressão (3.19).

2. Distribuição a posteriori condicional completa de $\boldsymbol{\lambda}_{1}$

De (3.30), a distribuição a posteriori condicional completa de $\boldsymbol{\lambda}_{1}$ é 


$$
\begin{aligned}
& \pi\left(\boldsymbol{\lambda}_{1} \mid \beta_{1}, \boldsymbol{\gamma}_{1}, \boldsymbol{\beta}_{2}, \boldsymbol{\gamma}_{2}, \boldsymbol{\lambda}_{2}, \theta, \boldsymbol{b}, \boldsymbol{E}^{*}, D_{o b s}\right) \propto \prod_{l=1}^{L} \prod_{i=1}^{n_{l}}\left[h_{1}\left(y_{E i l} \mid \boldsymbol{\lambda}_{1}\right)\left(H_{1}\left(y_{E i l} \mid \boldsymbol{\lambda}_{1}\right)\right)^{\theta-1}\right. \\
& \times \exp \left\{-\left[\left(H_{1}\left(y_{E i l} \mid \boldsymbol{\lambda}_{1}\right) \mathrm{e}^{K_{E i l}+b_{l}}\right)^{\theta}+\left(H_{2}\left(y_{G i l} \mid \boldsymbol{\lambda}_{2}\right) \mathrm{e}^{K_{G i l}+b_{l}}\right)^{\theta}\right]^{\frac{1}{\theta}}\right\}\left[\left(H_{1}\left(y_{E i l} \mid \boldsymbol{\lambda}_{1}\right) \mathrm{e}^{K_{E i l}+b_{l}}\right)^{\theta}\right. \\
& \left.+\left(H_{2}\left(y_{G i l} \mid \boldsymbol{\lambda}_{2}\right) \mathrm{e}^{K_{G i l}+b_{l}}\right)^{\theta}\right]^{\frac{1}{\theta}-2}\left(\theta-1+\left[\left(H_{1}\left(y_{E i l} \mid \boldsymbol{\lambda}_{1}\right) \mathrm{e}^{K_{E i l}+b_{l}}\right)^{\theta}+\left(H_{2}\left(y_{G i l} \mid \boldsymbol{\lambda}_{2}\right)\right.\right.\right. \\
& \left.\left.\left.\left.\times \mathrm{e}^{K_{G i l}+b_{l}}\right)^{\theta}\right]^{\frac{1}{\theta}}\right)\right]^{\mathbb{I}\left\{\begin{array}{c}
d_{i l}=1 \\
v_{i l}=1
\end{array}\right.}\left[h _ { 1 } ( y _ { E i l } | \boldsymbol { \lambda } _ { 1 } ) \operatorname { e x p } \left\{-H_{1}\left(y_{E i l} \mid \boldsymbol{\lambda}_{1}\right) \mathrm{e}^{K_{E i l}+b_{l}}-\left[\left(H_{1}\left(y_{E i l} \mid \boldsymbol{\lambda}_{1}\right)\right.\right.\right.\right. \\
& \left.\left.\left.\times \mathrm{e}^{K_{E i l}+b_{l}}\right)^{\theta}+\left(H_{2}\left(y_{G i l} \mid \boldsymbol{\lambda}_{2}\right) \mathrm{e}^{K_{G i l}+b_{l}}\right)^{\theta}\right]^{\frac{1}{\theta}}\right\}\left[\left(H_{1}\left(y_{E i l} \mid \boldsymbol{\lambda}_{1}\right) \mathrm{e}^{K_{E i l}+b_{l}}\right)^{\theta}+\left(H_{2}\left(y_{G i l} \mid \boldsymbol{\lambda}_{2}\right)\right.\right. \\
& \left.\left.\left.\times \mathrm{e}^{K_{G i l}+b_{l}}\right)^{\theta}\right]^{\frac{1}{\theta}-1}\right]^{\mathbb{I}\left\{\begin{array}{c}
d_{i l}=1 \\
v_{i l}=0
\end{array}\right.} \exp \left\{-E_{i l}^{*} \mathbb{I}\left\{\begin{array}{l}
d_{i l}=0 \\
v_{i l}=0
\end{array}\right\} H_{1}\left(y_{i l} \mid \boldsymbol{\lambda}_{1}\right) \mathrm{e}^{K_{E i l}+b_{l}}\right\} \prod_{j=1}^{J 1} \lambda_{1 j}^{a_{1 j}-1} \exp \left\{-b_{1 j} \lambda_{1 j}\right\},
\end{aligned}
$$

sendo que a função log-posteriori é dada por

$$
\begin{aligned}
& \mathcal{L}\left(\pi\left(\boldsymbol{\lambda}_{1} \mid \beta_{1}, \boldsymbol{\gamma}_{1}, \boldsymbol{\beta}_{2}, \boldsymbol{\gamma}_{2}, \boldsymbol{\lambda}_{2}, \theta, \boldsymbol{b}, \boldsymbol{E}^{*}, D_{\text {obs }}\right)\right)=\sum_{l=1}^{L} \sum_{i=1}^{n_{l}}\left(\mathbb{I}\left\{\begin{array}{c}
d_{i l}=1 \\
v_{i l}=1
\end{array}\right\}+\mathbb{I}\left\{\begin{array}{l}
d_{i l}=1 \\
v_{i l}=0
\end{array}\right\}\right) \\
& \times\left(\ln \left(h_{1}\left(y_{E i l} \mid \boldsymbol{\lambda}_{1}\right)\right)-\left[\left(H_{1}\left(y_{E i l} \mid \boldsymbol{\lambda}_{1}\right) \mathrm{e}^{K_{E i l}+b_{l}}\right)^{\theta}+\left(H_{2}\left(y_{G i l} \mid \boldsymbol{\lambda}_{2}\right) \mathrm{e}^{K_{G i l}+b_{l}}\right)^{\theta}\right]^{\frac{1}{\theta}}+\left(\frac{1}{\theta}-1\right)\right. \\
& \left.\times \ln \left(\left(H_{1}\left(y_{E i l} \mid \boldsymbol{\lambda}_{1}\right) \mathrm{e}^{K_{E i l}+b_{l}}\right)^{\theta}+\left(H_{2}\left(y_{G i l} \mid \boldsymbol{\lambda}_{2}\right) \mathrm{e}^{K_{G i l}+b_{l}}\right)^{\theta}\right)\right)+\sum_{l=1}^{L} \sum_{i=1}^{n_{l}} \mathbb{I}\left\{\begin{array}{l}
d_{i l}=1 \\
v_{i l}=1
\end{array}\right\}((\theta-1) \\
& \times \ln \left(H_{1}\left(y_{E i l} \mid \boldsymbol{\lambda}_{1}\right)\right)-\ln \left(\left(H_{1}\left(y_{E i l} \mid \boldsymbol{\lambda}_{1}\right) \mathrm{e}^{K_{E i l}+b_{l}}\right)^{\theta}+\left(H_{2}\left(y_{G i l} \mid \boldsymbol{\lambda}_{2}\right) \mathrm{e}^{K_{G i l}+b_{l}}\right)^{\theta}\right)+\ln (\theta-1 \\
& \left.\left.+\left[\left(H_{1}\left(y_{E i l} \mid \boldsymbol{\lambda}_{1}\right) \mathrm{e}^{K_{E i l}+b_{l}}\right)^{\theta}+\left(H_{2}\left(y_{G i l} \mid \boldsymbol{\lambda}_{2}\right) \mathrm{e}^{K_{G i l}+b_{l}}\right)^{\theta}\right]^{\frac{1}{\theta}}\right)\right)-\sum_{l=1}^{L} \sum_{i=1}^{n_{l}} \mathbb{I}\left\{\begin{array}{l}
d_{i l}=1 \\
v_{i l}=0
\end{array}\right\} H_{1}\left(y_{E i l} \mid \boldsymbol{\lambda}_{1}\right) \\
& \times \mathrm{e}^{K_{E i l}+b_{l}}-\sum_{l=1}^{L} \sum_{i=1}^{n_{l}} E_{i l}^{*} \mathbb{I}\left\{\begin{array}{l}
d_{i l}=0 \\
v_{i l}=0
\end{array}\right\} H_{1}\left(y_{i l} \mid \boldsymbol{\lambda}_{1}\right) \mathrm{e}^{K_{E i l}+b_{l}}+\sum_{j=1}^{J 1}\left(a_{1 j}-1\right) \log \left(\lambda_{1 j}\right)-b_{1 j} \lambda_{1 j} .
\end{aligned}
$$

3. Distribuição a posteriori condicional completa de $\boldsymbol{\lambda}_{2}$

De (3.30), a distribuição a posteriori condicional completa de $\boldsymbol{\lambda}_{2}$ é

$$
\begin{aligned}
& \pi\left(\boldsymbol{\lambda}_{2} \mid \beta_{1}, \gamma_{1}, \boldsymbol{\lambda}_{1}, \boldsymbol{\beta}_{2}, \boldsymbol{\gamma}_{2}, \theta, \boldsymbol{b}, D_{o b s}\right) \propto \prod_{l=1}^{L} \prod_{i=1}^{n_{l}}\left[h_{2}\left(y_{G i l} \mid \boldsymbol{\lambda}_{2}\right)\left(H_{2}\left(y_{G i l} \mid \boldsymbol{\lambda}_{2}\right)\right)^{\theta-1}\right. \\
& \times \exp \left\{-\left[\left(H_{1}\left(y_{E i l} \mid \boldsymbol{\lambda}_{1}\right) \mathrm{e}^{K_{E i l}+b_{l}}\right)^{\theta}+\left(H_{2}\left(y_{G i l} \mid \boldsymbol{\lambda}_{2}\right) \mathrm{e}^{K_{G i l}+b_{l}}\right)^{\theta}\right]^{\frac{1}{\theta}}\right\}\left[\left(H_{1}\left(y_{E i l} \mid \boldsymbol{\lambda}_{1}\right) \mathrm{e}^{K_{E i l}+b_{l}}\right)^{\theta}\right. \\
& \left.+\left(H_{2}\left(y_{G i l} \mid \boldsymbol{\lambda}_{2}\right) \mathrm{e}^{K_{G i l}+b_{l}}\right)^{\theta}\right]^{\frac{1}{\theta}-2}\left(\theta-1+\left[\left(H_{1}\left(y_{E i l} \mid \boldsymbol{\lambda}_{1}\right) \mathrm{e}^{K_{E i l}+b_{l}}\right)^{\theta}+\left(H_{2}\left(y_{G i l} \mid \boldsymbol{\lambda}_{2}\right)\right.\right.\right. \\
& \left.\left.\left.\left.\times \mathrm{e}^{K_{G i l}+b_{l}}\right)^{\theta}\right]^{\frac{1}{\theta}}\right)\right]^{\mathbb{I}\left\{\begin{array}{c}
d_{i l}=1 \\
v_{i l}=1
\end{array}\right.}\left[\exp \left\{-\left[\left(H_{1}\left(y_{E i l} \mid \boldsymbol{\lambda}_{1}\right) \mathrm{e}^{K_{E i l}+b_{l}}\right)^{\theta}+\left(H_{2}\left(y_{G i l} \mid \boldsymbol{\lambda}_{2}\right) \mathrm{e}^{K_{G i l}+b_{l}}\right)^{\theta}\right]^{\frac{1}{\theta}}\right\}\right.
\end{aligned}
$$


$\left.\times\left[\left(H_{1}\left(y_{E i l} \mid \boldsymbol{\lambda}_{1}\right) \mathrm{e}^{K_{E i l}+b_{l}}\right)^{\theta}+\left(H_{2}\left(y_{G i l} \mid \boldsymbol{\lambda}_{2}\right) \mathrm{e}^{K_{G i l}+b_{l}}\right)^{\theta}\right]^{\frac{1}{\theta}-1}\right]^{\mathbb{I}\left\{\prod_{j=1}^{d_{i l}=1}=0\right.} \prod_{j 2}^{J 2} \lambda_{2 j}^{a_{2 j}-1} \exp \left\{-b_{2 j} \lambda_{2 j}\right\}$,

em que a função log-posteriori é dada por

$$
\begin{aligned}
& \mathcal{L}\left(\pi\left(\boldsymbol{\lambda}_{2} \mid \beta_{1}, \boldsymbol{\gamma}_{1}, \boldsymbol{\lambda}_{1}, \boldsymbol{\beta}_{2}, \boldsymbol{\gamma}_{2}, \theta, \boldsymbol{b}, D_{\text {obs }}\right)\right)=\sum_{l=1}^{L} \sum_{i=1}^{n_{l}}\left(\mathbb{I}\left\{\begin{array}{l}
d_{i l}=1 \\
v_{i l}=1
\end{array}\right\}+\mathbb{I}\left\{\begin{array}{l}
d_{i l}=1 \\
v_{i l}=0
\end{array}\right\}\right) \\
& \times\left(-\left[\left(H_{1}\left(y_{E i l} \mid \boldsymbol{\lambda}_{1}\right) \mathrm{e}^{K_{E i l}+b_{l}}\right)^{\theta}+\left(H_{2}\left(y_{G i l} \mid \boldsymbol{\lambda}_{2}\right) \mathrm{e}^{K_{G i l}+b_{l}}\right)^{\theta}\right]^{\frac{1}{\theta}}+\left(\frac{1}{\theta}-1\right) \ln \left(\left(H_{1}\left(y_{E i l} \mid \boldsymbol{\lambda}_{1}\right)\right.\right.\right. \\
& \left.\left.\left.\times \mathrm{e}^{K_{E i l}+b_{l}}\right)^{\theta}+\left(H_{2}\left(y_{G i l} \mid \boldsymbol{\lambda}_{2}\right) \mathrm{e}^{K_{G i l}+b_{l}}\right)^{\theta}\right)\right)+\sum_{l=1}^{L} \sum_{i=1}^{n_{l}} \mathbb{I}\left\{\begin{array}{l}
d_{i l}=1 \\
v_{i l}=1
\end{array}\right\}\left(\ln \left(h_{2}\left(y_{G i l} \mid \boldsymbol{\lambda}_{2}\right)\right)+(\theta-1)\right. \\
& \times \ln \left(H_{2}\left(y_{G i l} \mid \boldsymbol{\lambda}_{2}\right)\right)-\ln \left(\left(H_{1}\left(y_{E i l} \mid \boldsymbol{\lambda}_{1}\right) \mathrm{e}^{K_{E i l}+b_{l}}\right)^{\theta}+\left(H_{2}\left(y_{G i l} \mid \boldsymbol{\lambda}_{2}\right) \mathrm{e}^{K_{G i l}+b_{l}}\right)^{\theta}\right)+\ln (\theta-1 \\
& \left.\left.\left.+\left[\left(H_{1}\left(y_{E i l} \mid \boldsymbol{\lambda}_{1}\right) \mathrm{e}^{K_{E i l}+b_{l}}\right)^{\theta}+\left(H_{2}\left(y_{G i l} \mid \boldsymbol{\lambda}_{2}\right) \mathrm{e}^{K_{G i l}+b_{l}}\right)^{\theta}\right)\right]^{\frac{1}{\theta}}\right)\right)-\sum_{j=1}^{J 2}\left(a_{2 j}-1\right) \ln \left(\lambda_{2 j}\right)-b_{2 j} \lambda_{2 j} .
\end{aligned}
$$

4. Distribuição a posteriori condicional completa de $\left(\beta_{0}, \gamma_{0}\right)$

A distribuição condicional completa de $\left(\beta_{0}, \gamma_{0}\right)$ corresponde com a mesma expressão da cópula de Clayton dada por (3.20).

5. Distribuição a posteriori condicional completa de $\left(\beta_{1}, \gamma_{1}\right)$

Relembrando que $K_{E i l}=A_{i l} \beta_{1}+\boldsymbol{x}_{i l}^{\top} \boldsymbol{\gamma}_{1}$ é preditor linear dos efeitos fixos e pela independência entre parâmetros, de (3.30) observa-se que a distribição a posteriori condicional completa de $\left(\beta_{1}, \gamma_{1}\right)$ é dada por

$$
\begin{aligned}
& \pi\left(\beta_{1}, \boldsymbol{\gamma}_{1} \mid \boldsymbol{\lambda}_{1}, \boldsymbol{\beta}_{2}, \boldsymbol{\gamma}_{2}, \boldsymbol{\lambda}_{2}, \theta, \boldsymbol{b}, \boldsymbol{E}^{*}, D_{o b s}\right) \propto \prod_{l=1}^{L} \prod_{i=1}^{n_{l}}\left[\operatorname { e x p } \left\{\theta K_{E i l}-\left[\left(H_{1}\left(y_{E i l} \mid \boldsymbol{\lambda}_{1}\right)\right.\right.\right.\right. \\
& \left.\left.\left.\times \mathrm{e}^{K_{E i l}+b_{l}}\right)^{\theta}+\left(H_{2}\left(y_{G i l} \mid \boldsymbol{\lambda}_{2}\right) \mathrm{e}^{K_{G i l}+b_{l}}\right)^{\theta}\right]^{\frac{1}{\theta}}\right\}\left[\left(H_{1}\left(y_{E i l} \mid \boldsymbol{\lambda}_{1}\right) \mathrm{e}^{K_{E i l}+b_{l}}\right)^{\theta}+\left(H_{2}\left(y_{G i l} \mid \boldsymbol{\lambda}_{2}\right)\right.\right. \\
& \left.\left.\left.\left.\times \mathrm{e}^{K_{G i l}+b_{l}}\right)^{\theta}\right]^{\frac{1}{\theta}-2}\left(\theta-1+\left[\left(H_{1}\left(y_{E i l} \mid \boldsymbol{\lambda}_{1}\right) \mathrm{e}^{K_{E i l}+b_{l}}\right)^{\theta}+\left(H_{2}\left(y_{G i l} \mid \boldsymbol{\lambda}_{2}\right) \mathrm{e}^{K_{G i l}+b_{l}}\right)^{\theta}\right]^{\frac{1}{\theta}}\right)\right]^{\mathbb{I}\left\{d_{i l}^{d_{i l}=1}\right\}}\right\} \\
& \times\left[\operatorname { e x p } \left\{K_{E i l}-H_{1}\left(y_{E i l} \mid \boldsymbol{\lambda}_{1}\right) \mathrm{e}^{K_{E i l}+b_{l}}-\left[\left(H_{1}\left(y_{E i l} \mid \boldsymbol{\lambda}_{1}\right) \mathrm{e}^{K_{E i l}+b_{l}}\right)^{\theta}+\left(H_{2}\left(y_{G i l} \mid \boldsymbol{\lambda}_{2}\right)\right.\right.\right.\right. \\
& \left.\left.\left.\left.\times \mathrm{e}^{K_{G i l}+b_{l}}\right)^{\theta}\right]^{\frac{1}{\theta}}\right\}\left[\left(H_{1}\left(y_{E i l} \mid \boldsymbol{\lambda}_{1}\right) \mathrm{e}^{K_{E i l}+b_{l}}\right)^{\theta}+\left(H_{2}\left(y_{G i l} \mid \boldsymbol{\lambda}_{2}\right) \mathrm{e}^{K_{G i l}+b_{l}}\right)^{\theta}\right]^{\frac{1}{\theta}-1}\right]^{\left.\mathbb{I} d_{v_{i l}=1}^{d_{i l}=0}\right\}} \\
& \times \exp \left\{-E_{i l}^{*} \mathbb{I}\left\{\begin{array}{l}
d_{i l}=0 \\
v_{i l}=0
\end{array} H_{1}\left(y_{i l} \mid \boldsymbol{\lambda}_{1}\right) \mathrm{e}^{K_{E i l}+b_{l}}\right\} \exp \left\{-0,5\left(\beta_{1}, \boldsymbol{\gamma}_{1}^{\top}\right)^{\top} \boldsymbol{\Sigma}_{1}^{-1}\left(\beta_{1}, \boldsymbol{\gamma}_{1}^{\top}\right)\right\},\right.
\end{aligned}
$$

e a função log-posteriori é dada por 


$$
\begin{aligned}
& \mathcal{L}\left(\pi\left[\beta_{1}, \boldsymbol{\gamma}_{1} \mid \boldsymbol{\lambda}_{1}, \boldsymbol{\beta}_{2}, \boldsymbol{\gamma}_{2}, \boldsymbol{\lambda}_{2}, \theta, \boldsymbol{b}, \boldsymbol{E}^{*}, D_{o b s}, b_{l}\right]\right) \propto \sum_{l=1}^{L} \sum_{i=1}^{n_{l}}\left(\mathbb{I}\left\{\begin{array}{c}
d_{i l}=1 \\
v_{i l}=1
\end{array}\right\}+\mathbb{I}\left\{\begin{array}{l}
d_{i l}=1 \\
v_{i l}=0
\end{array}\right\}\right) \\
& \times\left(-\left[\left(H_{1}\left(y_{E i l} \mid \boldsymbol{\lambda}_{1}\right) \mathrm{e}^{K_{E i l}+b_{l}}\right)^{\theta}+\left(H_{2}\left(y_{G i l} \mid \boldsymbol{\lambda}_{2}\right) \mathrm{e}^{K_{G i l}+b_{l}}\right)^{\theta}\right]^{\frac{1}{\theta}}+\left(\frac{1}{\theta}-1\right) \ln \left(\left(H_{1}\left(y_{E i l} \mid \boldsymbol{\lambda}_{1}\right)\right.\right.\right. \\
& \left.\left.\left.\times \mathrm{e}^{K_{E i l}+b_{l}}\right)^{\theta}+\left(H_{2}\left(y_{G i l} \mid \boldsymbol{\lambda}_{2}\right) \mathrm{e}^{K_{G i l}+b_{l}}\right)^{\theta}\right)\right)+\sum_{l=1}^{L} \sum_{i=1}^{n_{l}} \mathbb{I}\left\{\begin{array}{l}
d_{i l}=1 \\
v_{i l}=1
\end{array}\right\}\left(\theta K_{E i l}-\ln \left(\left(H_{1}\left(y_{E i l} \mid \boldsymbol{\lambda}_{1}\right)\right.\right.\right. \\
& \left.\left.\times \mathrm{e}^{K_{E i l}+b_{l}}\right)^{\theta}+\left(H_{2}\left(y_{G i l} \mid \boldsymbol{\lambda}_{2}\right) \mathrm{e}^{K_{G i l}+b_{l}}\right)^{\theta}\right)+\ln \left(\theta-1+\left[\left(H_{1}\left(y_{E i l} \mid \boldsymbol{\lambda}_{1}\right) \mathrm{e}^{K_{E i l}+b_{l}}\right)^{\theta}\right.\right. \\
& \left.\left.\left.+\left(H_{2}\left(y_{G i l} \mid \boldsymbol{\lambda}_{2}\right) \mathrm{e}^{K_{G i l}+b_{l}}\right)^{\theta}\right]^{\frac{1}{\theta}}\right)\right)+\sum_{l=1}^{L} \sum_{i=1}^{n_{l}} \mathbb{I}\left\{\begin{array}{c}
d_{i l}=1 \\
v_{i l}=0
\end{array}\right\}\left(K_{E i l}-H_{1}\left(y_{E i l} \mid \boldsymbol{\lambda}_{1}\right) \mathrm{e}^{K_{E i l}+b_{l}}\right) \\
& -\sum_{l=1}^{L} \sum_{i=1}^{n_{l}} E_{i l}^{*} \mathbb{I}\left\{\begin{array}{l}
d_{i l}=0 \\
v_{i l}=0
\end{array}\right\} H_{1}\left(y_{i l} \mid \boldsymbol{\lambda}_{1}\right) \mathrm{e}^{K_{E i l}+b_{l}}-0,5\left(\beta_{1}, \boldsymbol{\gamma}_{1}^{\top}\right)^{\top} \boldsymbol{\Sigma}_{1}^{-1}\left(\beta_{1}, \boldsymbol{\gamma}_{1}^{\top}\right) .
\end{aligned}
$$

6. Distribuição a posteriori marginal de $\left(\beta_{2}, \gamma_{2}\right)$

De (3.30) e considerando o preditor linear dos efeitos fixos $K_{G i l}=A_{i l} \beta_{21}+V_{i l}(1-$ $\left.A_{i l}\right) \beta_{22}+\boldsymbol{z}_{i l}^{\top} \gamma_{2}$ além da independência entre parâmetros, tem-se que a distribuição a posteriori condicional completa de $\left(\boldsymbol{\beta}_{2}, \boldsymbol{\gamma}_{2}\right)$ é

$$
\begin{aligned}
& \pi\left(\boldsymbol{\beta}_{2}, \boldsymbol{\gamma}_{2} \mid \beta_{1}, \boldsymbol{\gamma}_{1}, \boldsymbol{\lambda}_{1}, \boldsymbol{\lambda}_{2}, \theta, D_{o b s}, b_{l}\right) \propto \prod_{l=1}^{L} \prod_{i=1}^{n_{l}}\left[\operatorname { e x p } \left\{\theta K_{G i l}-\left[\left(H_{1}\left(y_{E i l} \mid \boldsymbol{\lambda}_{1}\right) \mathrm{e}^{K_{E i l}+b_{l}}\right)^{\theta}\right.\right.\right. \\
& \left.\left.+\left(H_{2}\left(y_{G i l} \mid \boldsymbol{\lambda}_{2}\right) \mathrm{e}^{K_{G i l}+b_{l}}\right)^{\theta}\right]^{\frac{1}{\theta}}\right\}\left[\left(H_{1}\left(y_{E i l} \mid \boldsymbol{\lambda}_{1}\right) \mathrm{e}^{K_{E i l}+b_{l}}\right)^{\theta}+\left(H_{2}\left(y_{G i l} \mid \boldsymbol{\lambda}_{2}\right) \mathrm{e}^{K_{G i l}+b_{l}}\right)^{\theta}\right]^{\frac{1}{\theta}-2} \\
& \left.\times\left(\theta-1+\left[\left(H_{1}\left(y_{E i l} \mid \boldsymbol{\lambda}_{1}\right) \mathrm{e}^{K_{E i l}+b_{l}}\right)^{\theta}+\left(H_{2}\left(y_{G i l} \mid \boldsymbol{\lambda}_{2}\right) \mathrm{e}^{K_{G i l}+b_{l}}\right)^{\theta}\right]^{\frac{1}{\theta}}\right)\right]^{\mathbb{I}\left\{d_{i l l}^{d_{i l}=1}\right\}} \\
& \times\left[\operatorname { e x p } \{ - [ ( H _ { 1 } ( y _ { E i l } | \boldsymbol { \lambda } _ { 1 } ) \mathrm { e } ^ { K _ { E i l } + b _ { l } } ) ^ { \theta } + ( H _ { 2 } ( y _ { G i l } | \boldsymbol { \lambda } _ { 2 } ) \mathrm { e } ^ { K _ { G i l } + b _ { l } } ) ^ { \theta } ] ^ { \frac { 1 } { \theta } } \} \left[\left(H_{1}\left(y_{E i l} \mid \boldsymbol{\lambda}_{1}\right) \mathrm{e}^{K_{E i l}+b_{l}}\right)^{\theta}\right.\right. \\
& \left.\left.+\left(H_{2}\left(y_{G i l} \mid \boldsymbol{\lambda}_{2}\right) \mathrm{e}^{K_{G i l}+b_{l}}\right)^{\theta}\right]^{\frac{1}{\theta}-1}\right]^{\mathbb{I}\left\{\left\{_{v_{i l}=0}^{d_{i l}=1}\right\}\right.} \exp \left\{-0,5\left(\boldsymbol{\beta}_{2}^{\top}, \boldsymbol{\gamma}_{2}^{\top}\right)^{\top} \boldsymbol{\Sigma}_{2}^{-1}\left(\boldsymbol{\beta}_{2}^{\top}, \boldsymbol{\gamma}_{2}^{\top}\right)\right\},
\end{aligned}
$$

com a função log-posteriori dada por

$$
\begin{aligned}
& \mathcal{L}\left(\pi\left(\boldsymbol{\beta}_{2}, \boldsymbol{\gamma}_{2} \mid \beta_{1}, \boldsymbol{\gamma}_{1}, \boldsymbol{\lambda}_{1}, \boldsymbol{\lambda}_{2}, \boldsymbol{b}, \theta, D_{o b s}\right)\right)=\sum_{l=1}^{L} \sum_{i=1}^{n_{l}}\left(\mathbb{I}\left\{\begin{array}{c}
d_{i l}=1 \\
v_{i l}=1
\end{array}\right\}+\mathbb{I}\left\{\begin{array}{l}
d_{i l}=1 \\
v_{i l}=0
\end{array}\right\}\right)\left(-\left[\left(H_{1}\left(y_{E i l} \mid \boldsymbol{\lambda}_{1}\right)\right.\right.\right. \\
& \left.\left.\times \mathrm{e}^{K_{E i l}+b_{l}}\right)^{\theta}+\left(H_{2}\left(y_{G i l} \mid \boldsymbol{\lambda}_{2}\right) \mathrm{e}^{K_{G i l}+b_{l}}\right)^{\theta}\right]^{\frac{1}{\theta}}+\left(\frac{1}{\theta}-1\right) \ln \left(\left(H_{1}\left(y_{E i l} \mid \boldsymbol{\lambda}_{1}\right) \mathrm{e}^{K_{E i l}+b_{l}}\right)^{\theta}\right. \\
& \left.\left.+\left(H_{2}\left(y_{G i l} \mid \boldsymbol{\lambda}_{2}\right) \mathrm{e}^{K_{G i l}+b_{l}}\right)^{\theta}\right)\right)+\sum_{l=1}^{L} \sum_{i=1}^{n_{l}} \mathbb{I}\left\{\begin{array}{l}
d_{i l}=1 \\
v_{i l}=1
\end{array}\right\}\left(\theta K_{G i l}-\ln \left(\left(H_{1}\left(y_{E i l} \mid \boldsymbol{\lambda}_{1}\right) \mathrm{e}^{K_{E i l}+b_{l}}\right)^{\theta}\right.\right.
\end{aligned}
$$




$$
\begin{aligned}
& \left.+\left(H_{2}\left(y_{G i l} \mid \boldsymbol{\lambda}_{2}\right) \mathrm{e}^{K_{G i l}+b_{l}}\right)^{\theta}\right)+\ln \left(\theta-1+\left[\left(H_{1}\left(y_{E i l} \mid \boldsymbol{\lambda}_{1}\right) \mathrm{e}^{K_{E i l}+b_{l}}\right)^{\theta}+\left(H_{2}\left(y_{G i l} \mid \boldsymbol{\lambda}_{2}\right)\right.\right.\right. \\
& \left.\left.\left.\left.\left.\times \mathrm{e}^{K_{G i l}+b_{l}}\right)^{\theta}\right)\right]^{\frac{1}{\theta}}\right)\right)-0,5\left(\boldsymbol{\beta}_{2}^{\top}, \boldsymbol{\gamma}_{2}^{\top}\right)^{\top} \boldsymbol{\Sigma}_{2}^{-1}\left(\boldsymbol{\beta}_{2}^{\top}, \boldsymbol{\gamma}_{2}^{\top}\right) .
\end{aligned}
$$

7. Distribuição a posteriori condicional completa da variável latente $\boldsymbol{E}^{*}$

Esta distribuição se conserva como na cópula de Clayton, portanto aqui é omitida, veja (3.21).

8. Distribuição a posteriori condicional completa do parâmetro da cópula $\theta$ De (3.30) e pela independência entre parâmetros, tem-se que a distribuição $a$ posteriori condicional completa para $\theta$ fica expressa como

$$
\begin{aligned}
& \pi\left(\theta \mid \beta_{1}, \gamma_{1}, \boldsymbol{\lambda}_{1}, \boldsymbol{\beta}_{2}, \gamma_{2}, \boldsymbol{\lambda}_{2}, \boldsymbol{b}, \boldsymbol{E}^{*}, D_{o b s}\right) \propto \prod_{l=1}^{L} \prod_{i=1}^{n_{l}}\left[\left(H_{1}\left(y_{E i l} \mid \boldsymbol{\lambda}_{1}\right) H_{2}\left(y_{G i l} \mid \boldsymbol{\lambda}_{2}\right)\right)^{\theta}\right. \\
& \times \exp \left\{\theta\left(K_{E i l}+K_{G i l}+2 b_{l}\right)-\left[\left(H_{1}\left(y_{E i l} \mid \boldsymbol{\lambda}_{1}\right) \mathrm{e}^{K_{E i l}+b_{l}}\right)^{\theta}+\left(H_{2}\left(y_{G i l} \mid \boldsymbol{\lambda}_{2}\right) \mathrm{e}^{K_{G i l}+b_{l}}\right)^{\theta}\right]^{\frac{1}{\theta}}\right\} \\
& \times\left[\left(H_{1}\left(y_{E i l} \mid \boldsymbol{\lambda}_{1}\right) \mathrm{e}^{K_{E i l}+b_{l}}\right)^{\theta}+\left(H_{2}\left(y_{G i l} \mid \boldsymbol{\lambda}_{2}\right) \mathrm{e}^{K_{G i l}+b_{l}}\right)^{\theta}\right]^{\frac{1}{\theta}-2}\left(\theta-1+\left[\left(H_{1}\left(y_{E i l} \mid \boldsymbol{\lambda}_{1}\right)\right.\right.\right. \\
& \left.\left.\left.\left.\times \mathrm{e}^{K_{E i l}+b_{l}}\right)^{\theta}+\left(H_{2}\left(y_{G i l} \mid \boldsymbol{\lambda}_{2}\right) \mathrm{e}^{K_{G i l}+b_{l}}\right)^{\theta}\right]^{\frac{1}{\theta}}\right)\right]^{\mathbb{I}\left\{\begin{array}{c}
d_{i l}=1 \\
v_{i l}=1
\end{array}\right\}}\left[\operatorname { e x p } \left\{-\left[\left(H_{1}\left(y_{E i l} \mid \boldsymbol{\lambda}_{1}\right) \mathrm{e}^{K_{E i l}+b_{l}}\right)^{\theta}\right.\right.\right. \\
& \left.\left.\left.+\left(H_{2}\left(y_{G i l} \mid \boldsymbol{\lambda}_{2}\right) \mathrm{e}^{K_{G i l}+b_{l}}\right)^{\theta}\right]^{\frac{1}{\theta}}\right\}\left[\left(H_{1}\left(y_{E i l} \mid \boldsymbol{\lambda}_{1}\right) \mathrm{e}^{K_{E i l}+b_{l}}\right)^{\theta}+\left(H_{2}\left(y_{G i l} \mid \boldsymbol{\lambda}_{2}\right) \mathrm{e}^{K_{G i l}+b_{l}}\right)^{\theta}\right]^{\frac{1}{\theta}-1}\right]^{\mathbb{I}\left\{\begin{array}{c}
d_{i l}=1 \\
v_{i l}=0
\end{array}\right\}} \\
& \times \exp \left\{-\frac{\theta^{2}}{2 \sigma_{\theta}^{2}}\right\}
\end{aligned}
$$

e a função log-posteriori por

$$
\begin{aligned}
& \mathcal{L}\left(\pi\left(\theta \mid \beta_{1}, \boldsymbol{\gamma}_{1}, \boldsymbol{\lambda}_{1}, \boldsymbol{\beta}_{2}, \boldsymbol{\gamma}_{2}, \boldsymbol{\lambda}_{2}, \boldsymbol{b}, \boldsymbol{E}^{*}, D_{o b s}\right)\right)=\sum_{l=1}^{L} \sum_{i=1}^{n_{l}}\left(\mathbb{I}\left\{\begin{array}{c}
d_{i l}=1 \\
v_{i l}=1
\end{array}\right\}+\mathbb{I}\left\{\begin{array}{c}
d_{i l}=1 \\
v_{i l}=0
\end{array}\right\}\right) \\
& \times\left(-\left[\left(H_{1}\left(y_{E i l} \mid \boldsymbol{\lambda}_{1}\right) \mathrm{e}^{K_{E i l}+b_{l}}\right)^{\theta}+\left(H_{2}\left(y_{G i l} \mid \boldsymbol{\lambda}_{2}\right) \mathrm{e}^{K_{G i l}+b_{l}}\right)^{\theta}\right]^{\frac{1}{\theta}}+\left(\frac{1}{\theta}-1\right) \ln \left(\left(H_{1}\left(y_{E i l} \mid \boldsymbol{\lambda}_{1}\right)\right.\right.\right. \\
& \left.\left.\left.\times \mathrm{e}^{K_{E i l}+b_{l}}\right)^{\theta}+\left(H_{2}\left(y_{G i l} \mid \boldsymbol{\lambda}_{2}\right) \mathrm{e}^{K_{G i l}+b_{l}}\right)^{\theta}\right)\right)+\sum_{l=1}^{L} \sum_{i=1}^{n_{l}} \mathbb{I}\left\{\begin{array}{l}
d_{i l}=1 \\
v_{i l}=1
\end{array}\right\}\left(\theta \ln \left(H_{1}\left(y_{E i l} \mid \boldsymbol{\lambda}_{1}\right) H_{2}\left(y_{G i l} \mid \boldsymbol{\lambda}_{2}\right)\right)\right. \\
& +\theta\left(K_{E i l}+K_{G i l}+2 b_{l}\right)-\ln \left(\left(H_{1}\left(y_{E i l} \mid \boldsymbol{\lambda}_{1}\right) \mathrm{e}^{K_{E i l}+b_{l}}\right)^{\theta}+\left(H_{2}\left(y_{G i l} \mid \boldsymbol{\lambda}_{2}\right) \mathrm{e}^{K_{G i l}+b_{l}}\right)^{\theta}\right)+\ln (\theta \\
& \left.\left.\left.-1+\left[\left(H_{1}\left(y_{E i l} \mid \boldsymbol{\lambda}_{1}\right) \mathrm{e}^{K_{E i l}+b_{l}}\right)^{\theta}+\left(H_{2}\left(y_{G i l} \mid \boldsymbol{\lambda}_{2}\right) \mathrm{e}^{K_{G i l}+b_{l}}\right)^{\theta}\right)\right]^{\frac{1}{\theta}}\right)\right)-\frac{\theta^{2}}{2 \sigma_{\theta}^{2}} .
\end{aligned}
$$

9. Distribuição a posteriori condicional completa do preditor linear do modelo logito $\alpha$

Esta distribuição também se conserva como na cópula de Clayton conforme (3.22).

10. Distribuição a posteriori condicional completa da variância do efeito aleatório $B$. Como na cópula de Clayton $[B \mid \boldsymbol{b}] \sim \operatorname{Gama} \operatorname{Inversa}\left(\frac{L}{2}+\kappa_{B} ; \frac{\sum_{l=1}^{L} b_{l}^{2}}{2}+\varsigma_{B}\right)$. 
11. Distribuição a posteriori condicional completa do vetor de efeitos aleatórios $\boldsymbol{b}$ Da distribuição a posteriori conjunta aumentada (3.30), tem-se que

$$
\begin{aligned}
& \pi\left[\boldsymbol{b} \mid \beta_{0}, \boldsymbol{\gamma}_{0}, \beta_{1}, \boldsymbol{\gamma}_{1}, \boldsymbol{\beta}_{2}, \boldsymbol{\gamma}_{2}, \boldsymbol{\lambda}_{0}, \boldsymbol{\lambda}_{1}, \boldsymbol{\lambda}_{2}, \theta, B, D_{o b s}\right] \propto \prod_{l=1}^{L} \prod_{i=1}^{n_{l}}\left[\operatorname { e x p } \left\{b_{l}-H_{0}\left(y_{i l} \mid \boldsymbol{\lambda}_{0}\right)\right.\right. \\
& \left.\left.\times \mathrm{e}^{K_{D i l}+b_{l}}\right\}\right]^{\mathbb{I}\left\{\begin{array}{c}
d_{i l}=0 \\
v_{i l}=1
\end{array}\right.}\left[\exp \left\{2 \theta b_{l}-\left[\left(H_{1}\left(y_{E i l} \mid \boldsymbol{\lambda}_{1}\right) \mathrm{e}^{K_{E i l}+b_{l}}\right)^{\theta}+\left(H_{2}\left(y_{G i l} \mid \boldsymbol{\lambda}_{2}\right) \mathrm{e}^{K_{G i l}+b_{l}}\right)^{\theta}\right]^{\frac{1}{\theta}}\right\}\right. \\
& \times\left[\left(H_{1}\left(y_{E i l} \mid \boldsymbol{\lambda}_{1}\right) \mathrm{e}^{K_{E i l}+b_{l}}\right)^{\theta}+\left(H_{2}\left(y_{G i l} \mid \boldsymbol{\lambda}_{2}\right) \mathrm{e}^{K_{G i l}+b_{l}}\right)^{\theta}\right]^{\frac{1}{\theta}-2}\left(\theta-1+\left[\left(H_{1}\left(y_{E i l} \mid \boldsymbol{\lambda}_{1}\right)\right.\right.\right. \\
& \left.\left.\left.\left.\times \mathrm{e}^{K_{E i l}+b_{l}}\right)^{\theta}+\left(H_{2}\left(y_{G i l} \mid \boldsymbol{\lambda}_{2}\right) \mathrm{e}^{K_{G i l}+b_{l}}\right)^{\theta}\right]^{\frac{1}{\theta}}\right)\right]^{\mathbb{I}\left\{\begin{array}{l}
d_{i l}=1 \\
v_{i l}=1
\end{array}\right\}}\left[\operatorname { e x p } \left\{b_{l}-H_{1}\left(y_{E i l} \mid \boldsymbol{\lambda}_{1}\right) \mathrm{e}^{K_{E i l}+b_{l}}\right.\right. \\
& \left.-\left[\left(H_{1}\left(y_{E i l} \mid \boldsymbol{\lambda}_{1}\right) \mathrm{e}^{K_{E i l}+b_{l}}\right)^{\theta}+\left(H_{2}\left(y_{G i l} \mid \boldsymbol{\lambda}_{2}\right) \mathrm{e}^{K_{G i l}+b_{l}}\right)^{\theta}\right]^{\frac{1}{\theta}}\right\}\left[\left(H_{1}\left(y_{E i l} \mid \boldsymbol{\lambda}_{1}\right) \mathrm{e}^{K_{E i l}+b_{l}}\right)^{\theta}\right. \\
& \left.\left.+\left(H_{2}\left(y_{G i l} \mid \boldsymbol{\lambda}_{2}\right) \mathrm{e}^{K_{G i l}+b_{l}}\right)^{\theta}\right]^{\frac{1}{\theta}-1}\right]^{\mathbb{I}\left\{\begin{array}{c}
d_{i l}=1 \\
v_{i l}=0
\end{array}\right.}\left[\operatorname { e x p } \{ - E _ { i l } ^ { * } H _ { 1 } ( y _ { i l } | \boldsymbol { \lambda } _ { 1 } ) \mathrm { e } ^ { K _ { E i l } + b _ { l } } \} \operatorname { e x p } \left\{-\left(1-E_{i l}^{*}\right)\right.\right. \\
& \left.\left.\times H_{0}\left(y_{i l} \mid \boldsymbol{\lambda}_{0}\right) \mathrm{e}^{K_{D i l}+b_{l}}\right\}\right]^{\mathbb{I}\left\{d_{i l}^{d_{i l}=0}\right\}} \exp \left\{-\frac{b_{l}^{2}}{2 B}\right\},
\end{aligned}
$$

com a função log-posteriori expressa como

$$
\begin{aligned}
& \mathcal{L}\left(\pi\left(\boldsymbol{b} \mid \beta_{0}, \boldsymbol{\gamma}_{0}, \beta_{1}, \boldsymbol{\gamma}_{1}, \boldsymbol{\beta}_{2}, \boldsymbol{\gamma}_{2}, \boldsymbol{\lambda}_{0}, \boldsymbol{\lambda}_{1}, \boldsymbol{\lambda}_{2}, \theta, B, D_{o b s}\right)\right)=\sum_{l=1}^{L} \sum_{i=1}^{n_{l}} \mathbb{I}\left\{\begin{array}{l}
d_{i l}=0 \\
v_{i l}=1
\end{array}\right\}\left(b_{l}\right. \\
& \left.-H_{0}\left(y_{i l} \mid \boldsymbol{\lambda}_{0}\right) \mathrm{e}^{K_{D i l}+b_{l}}\right)+\sum_{l=1}^{L} \sum_{i=1}^{n_{l}}\left(\mathbb{I}\left\{\begin{array}{l}
d_{i l}=1 \\
v_{i l}=1
\end{array}\right\}+\mathbb{I}\left\{\begin{array}{l}
d_{i l}=1 \\
v_{i l}=0
\end{array}\right\}\right)\left(-\left[\left(H_{1}\left(y_{E i l} \mid \boldsymbol{\lambda}_{1}\right) \mathrm{e}^{K_{E i l}+b_{l}}\right)^{\theta}\right.\right. \\
& \left.+\left(H_{2}\left(y_{G i l} \mid \boldsymbol{\lambda}_{2}\right) \mathrm{e}^{K_{G i l}+b_{l}}\right)^{\theta}\right]^{\frac{1}{\theta}}+\left(\frac{1}{\theta}-1\right) \ln \left(\left(H_{1}\left(y_{E i l} \mid \boldsymbol{\lambda}_{1}\right) \mathrm{e}^{K_{E i l}+b_{l}}\right)^{\theta}+\left(H_{2}\left(y_{G i l} \mid \boldsymbol{\lambda}_{2}\right)\right.\right. \\
& \left.\left.\left.\times \mathrm{e}^{K_{G i l}+b_{l}}\right)^{\theta}\right)\right)+\sum_{l=1}^{L} \sum_{i=1}^{n_{l}} \mathbb{I}\left\{\begin{array}{l}
d_{i l}=1 \\
v_{i l}=1
\end{array}\right\}\left(2 \theta b_{l}-\ln \left(\left(H_{1}\left(y_{E i l} \mid \boldsymbol{\lambda}_{1}\right) \mathrm{e}^{K_{E i l}+b_{l}}\right)^{\theta}\right.\right. \\
& \left.+\left(H_{2}\left(y_{G i l} \mid \boldsymbol{\lambda}_{2}\right) \mathrm{e}^{K_{G i l}+b_{l}}\right)^{\theta}\right)+\ln \left(\theta-1+\left[\left(H_{1}\left(y_{E i l} \mid \boldsymbol{\lambda}_{1}\right) \mathrm{e}^{K_{E i l}+b_{l}}\right)^{\theta}+\left(H_{2}\left(y_{G i l} \mid \boldsymbol{\lambda}_{2}\right)\right.\right.\right. \\
& \left.\left.\left.\left.\left.\times \mathrm{e}^{K_{G i l}+b_{l}}\right)^{\theta}\right)\right]^{\frac{1}{\theta}}\right)\right)+\sum_{l=1}^{L} \sum_{i=1}^{n_{l}} \mathbb{I}\left\{\begin{array}{l}
d_{i l}=1 \\
v_{i l}=0
\end{array}\right\}\left(b_{l}-H_{1}\left(y_{E i l} \mid \boldsymbol{\lambda}_{1}\right) \mathrm{e}^{K_{E i l}+b_{l}}\right)-\sum_{l=1}^{L} \sum_{i=1}^{n_{l}} \mathbb{I}\left\{\begin{array}{l}
d_{i l}=0 \\
v_{i l}=0
\end{array}\right\} \\
& \times\left(E_{i l}^{*} H_{1}\left(y_{i l} \mid \boldsymbol{\lambda}_{1}\right) \mathrm{e}^{K_{E i l}+b_{l}}+\left(1-E_{i l}^{*}\right) H_{0}\left(y_{i l} \mid \boldsymbol{\lambda}_{0}\right) \mathrm{e}^{K_{D i l}+b_{l}}\right)-\sum_{l=1}^{L} \frac{b_{l}^{2}}{2 B} .
\end{aligned}
$$




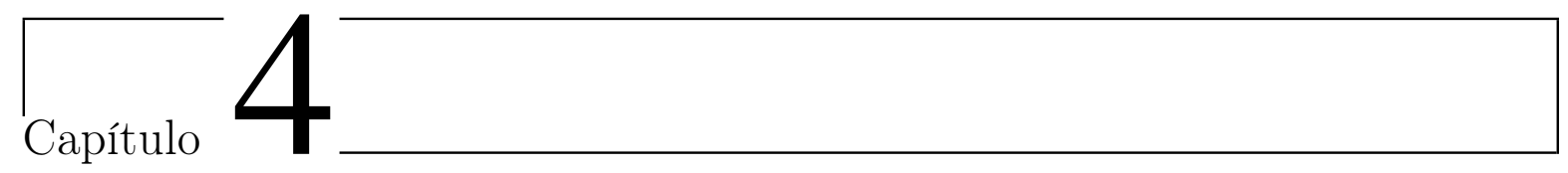

\section{Aspectos computacionais e estudo de simulação}

Neste capítulo é apresentado o esquema computacional da modelagem proposta. Para analisar o desempenho da modelagem por meio do amostrador de Gibbs, é preciso, na inferência bayesiana, amostrar da distribuição a posteriori completa dada em (3.14) para isso, o algortimo de Gibbs é implementado conforme descrito na Secção 4.1. Posteriormente, uma validação do método de estimação é efetuada através de um estudo de simulação na Secção 4.2. Finalmente resultados de tal estudo são apresentados segundo a cópula de Clayton. A programação é desenvolvida na linguagem $\mathrm{R}$ versão 3.2.4 (R Core Team, 2015).

\subsection{Amostragem}

Inicialmente são desenvolvidas funções preliminares com as quais obtém-se:

1. Os pontos $\left\{s_{k 0}, s_{k 1}, s_{k 2}, \cdots, s_{k J_{k}}\right\}, k=0,1,2$ da partição finita do tempo para calcular as funções de risco basal constantes por partes $h_{0}(t), h_{1}(t)$ e $h_{2}(t)$ segundo a quantidade de intervalos $J_{k}$. Tal partição foi implementada usando a função quantile em $\mathrm{R}$, determinando os intervalos segundo os quantis dos dados observados, sem as censuras, o que garante a obtenção de pelo menos uma falha em cada intervalo.

2. Uma matriz que contém as variáveis indicadoras dos $N$ indivíduos nos $J_{k}$ intervalos, $\delta_{i k j}, i=1, \cdots, n, k=0,1,2, j=1, \cdots, J_{k}$ e os comprimentos dos intervalos necessários para calcular a função de risco basal acumulada $H_{k}\left(t \mid \boldsymbol{\lambda}_{k}\right) k=0,1,2$ dada por (3.6).

3. Uma matriz de planejamento para o efeito aleatório, para o caso do modelo misto.

4. O período de aquecimento (burn-in) e espaçamento (thinning) das cadeias de Markov após amostragem da distribuição a posteriori (3.18).

5. A validação das condições do Teorema 1. A implementação foi feita utilizando a função rankMatrix do pacote Matrix. 
Estas funções preliminares estão ligadas a um programa principal que contém as especificações dos valores iniciais das cadeias de Markov, os hiperparâmetros das distribuições apriori e o algoritmo de Gibbs como descrito em seguida. Para este último são chamados os programas com a implementação das distribuições aposteriori condicionais completas.

Como comentado anteriormente, para obter amostras da distribuição a posteriori aumentada (3.14) é utilizado o amostrador de Gibbs. Assim, o esquema para amostrar o vetor de parâmetros $\boldsymbol{\xi}=\left(\boldsymbol{\alpha}^{\top}, \beta_{0}, \boldsymbol{\gamma}_{0}^{\top}, \boldsymbol{\lambda}_{0}^{\top}, \beta_{1}, \boldsymbol{\gamma}_{1}^{\top}, \boldsymbol{\lambda}_{1}^{\top}, \boldsymbol{\beta}_{2}^{\top}, \boldsymbol{\gamma}_{2}^{\top}, \boldsymbol{\lambda}_{2}^{\top}, \theta\right)^{\top}$, a variância do efeito aleatório $B$ e as variáveis latentes, $\boldsymbol{b}$ e $\boldsymbol{E}^{*}$, inseridas na distribuição a posteriori (3.12) é detalhado a seguir.

Passo 0. Considerar a iteração $r=0$ e escolher valores iniciais para os parâmetros

$$
\boldsymbol{\xi}^{(0)}=\left(\boldsymbol{\alpha}^{(0)},\left(\beta_{0}, \gamma_{0}\right)^{(0)}, \boldsymbol{\lambda}_{0}^{(0)},\left(\beta_{1}, \boldsymbol{\gamma}_{1}\right)^{(0)}, \boldsymbol{\lambda}_{1}^{(0)},\left(\boldsymbol{\beta}_{2}, \boldsymbol{\gamma}_{2}\right)^{(0)}, \boldsymbol{\lambda}_{2}^{(0)}, \theta^{(0)}\right), B^{(0)}, \boldsymbol{b}^{(0)}, \boldsymbol{E}^{*(0)}
$$

Neste ponto, para os valores iniciais foram ajustados modelos logito para $\boldsymbol{\alpha}^{(0)}$ e modelos de Cox para os coeficientes das regressões associados ao tempo até o óbito, tempo até a progressão e tempo até óbito após progressão, $\left(\beta_{0}, \boldsymbol{\gamma}_{0}\right)^{(0)},\left(\beta_{1}, \boldsymbol{\gamma}_{1}\right)^{(0)}$ e $\left(\boldsymbol{\beta}_{2}, \boldsymbol{\gamma}_{2}\right)^{(0)}$ respectivamente. Os efeitos aleatórios foram gerados como $\mathrm{N}(0,1)$, sendo $B^{(0)}=1$ sua variância. Para $\boldsymbol{E}^{*(0)}, \theta^{(0)}$ e as funções de risco basais iniciais $\left(\boldsymbol{\lambda}_{0}^{(0)}, \boldsymbol{\lambda}_{1}^{(0)}, \boldsymbol{\lambda}_{2}^{(0)}\right)$ foram amostrados valores das suas respectivas distribuições a posteriori marginais, condicionais nos valores iniciais dos modelos logito e Cox ajustados.

Para gerar amostras das distribuições a posteriori marginais (de todos os parâmetros), foi implementado o algoritmo de amostrador por fatias. Este amostrador, usado na implementação, encontra-se disponível no pacote MfUSampler da linguagem R.

Passo 1. Gerar $\boldsymbol{\xi}^{(r+1)}, B^{(r+1)}, \boldsymbol{b}^{(r+1)}$ e $\boldsymbol{E}^{*(r+1)}$, sendo

$$
\boldsymbol{\xi}^{(r+1)}=\left(\boldsymbol{\alpha}^{(r+1)},\left(\beta_{0}, \gamma_{0}\right)^{(r+1)}, \boldsymbol{\lambda}_{0}^{(r+1)},\left(\beta_{1}, \boldsymbol{\gamma}_{1}\right)^{(r+1)}, \boldsymbol{\lambda}_{1}^{(r+1)},\left(\boldsymbol{\beta}_{2}, \boldsymbol{\gamma}_{2}\right)^{(r+1)}, \boldsymbol{\lambda}_{2}^{(r+1)}, \theta^{(r+1)}\right),
$$

como segue:

- Gerar $\boldsymbol{\lambda}_{0}^{(r+1)} \sim \pi\left(\boldsymbol{\lambda}_{0}^{(r+1)} \mid\left(\beta_{0}, \gamma_{0}\right)^{(r)}, \boldsymbol{b}^{(r)}, \boldsymbol{E}^{*(r)}, D_{o b s}\right)$ com valor inicial $\boldsymbol{\lambda}_{0}^{(r)}$;

- Gerar $\boldsymbol{\lambda}_{1}^{(r+1)} \sim \pi\left(\boldsymbol{\lambda}_{1}^{(r+1)} \mid\left(\beta_{1}, \boldsymbol{\gamma}_{1}\right)^{(r)},\left(\boldsymbol{\beta}_{2}, \boldsymbol{\gamma}_{2}\right)^{(r)}, \boldsymbol{\lambda}_{2}^{(r)}, \theta^{(r)}, \boldsymbol{b}^{(r)}, \boldsymbol{E}^{*(r)}, D_{o b s}\right)$ com valor inicial $\boldsymbol{\lambda}_{1}^{(r)}$;

- Gerar $\boldsymbol{\lambda}_{2}^{(r+1)} \sim \pi\left(\boldsymbol{\lambda}_{2}^{(r+1)} \mid\left(\beta_{1}, \boldsymbol{\gamma}_{1}\right)^{(r)}, \boldsymbol{\lambda}_{1}^{(r+1)},\left(\boldsymbol{\beta}_{2}, \boldsymbol{\gamma}_{2}\right)^{(r)}, \theta^{(r)}, \boldsymbol{b}^{(r)}, \boldsymbol{E}^{*(r)}, D_{o b s}\right)$, com valor inicial $\boldsymbol{\lambda}_{2}^{(r)}$;

- $\operatorname{Gerar}\left(\beta_{0}, \gamma_{0}\right)^{(r+1)} \sim \pi\left(\left(\beta_{0}, \gamma_{0}\right)^{(r+1)} \mid \boldsymbol{\lambda}_{0}^{(r+1)}, \boldsymbol{b}^{(r)}, \boldsymbol{E}^{*(r)}, D_{o b s}\right)$, com valor inicial $\left(\beta_{0}, \gamma_{0}\right)^{(r)}$

- $\operatorname{Gerar}\left(\beta_{1}, \boldsymbol{\gamma}_{1}\right)^{(r+1)} \sim \pi\left(\left(\beta_{1}, \boldsymbol{\gamma}_{1}\right)^{(r+1)} \mid \boldsymbol{\lambda}_{1}^{(r+1)},\left(\boldsymbol{\beta}_{2}, \boldsymbol{\gamma}_{2}\right)^{(r)}, \boldsymbol{\lambda}_{2}^{(r+1)}, \theta^{(r)}, \boldsymbol{b}^{(r)}, \boldsymbol{E}^{*(r)}, D_{o b s}\right)$, com valor inicial $\left(\beta_{1}, \gamma_{1}\right)^{(r)}$; 
- Gerar $\left(\boldsymbol{\beta}_{2}, \boldsymbol{\gamma}_{2}\right)^{(r+1)} \sim \pi\left(\left(\boldsymbol{\beta}_{2}, \boldsymbol{\gamma}_{2}\right)^{(r+1)} \mid\left(\beta_{1}, \boldsymbol{\gamma}_{1}\right)^{(r+1)}, \boldsymbol{\lambda}_{1}^{(r+1)}, \boldsymbol{\lambda}_{2}^{(r+1)}, \theta^{(r)}, \boldsymbol{b}^{(r)}, \boldsymbol{E}^{*(r)}\right.$, $\left.D_{\text {obs }}\right)$, com valor inicial $\left(\boldsymbol{\beta}_{2}, \boldsymbol{\gamma}_{2}\right)^{(r)}$;

- Gerar $\boldsymbol{E}^{*(r+1)} \sim \pi\left(\boldsymbol{E}^{*(r+1)} \mid \boldsymbol{\alpha}^{(r)},\left(\beta_{0}, \boldsymbol{\gamma}_{0}\right)^{(r+1)}, \boldsymbol{\lambda}_{0}^{(r+1)},\left(\beta_{1}, \boldsymbol{\gamma}_{1}\right)^{(r+1)}, \boldsymbol{\lambda}_{1}^{(r+1)}, \theta^{(r)}, \boldsymbol{b}^{(r)}\right.$, $\left.D_{\text {obs }}\right)$, com distribuição Bernoulli com parâmetro $p$, em que $p=a /(a+b)$. Como descrito no item 7 da Seção 3.2, a distribuição a posteriori marginal de $\boldsymbol{E}^{*}$ pode ser interpretada como $f\left(E^{*}\right)=\left(a^{E^{*}}\right)\left(b^{1-E^{*}}\right) \operatorname{com} a=\frac{\exp \left\{K_{P i l}-H_{1}\left(y_{i l} \mid \boldsymbol{\lambda}_{1}\right) \mathrm{e}^{K_{E i l}+b_{l}}\right\}}{1+\mathrm{e}^{K_{P i l}}}$ e $b=\frac{\exp \left\{-H_{0}\left(y_{i l} \mid \boldsymbol{\lambda}_{0}\right) \mathrm{e}^{K_{D i l}+b_{l}}\right\}}{1+\mathrm{e}^{K}{ }^{P i l}}$ para o $i$-ésimo indivíduo no $l$-ésimo grupo;

- $\operatorname{Gerar} \theta^{(r+1)} \sim \pi\left(\theta^{(r+1)} \mid\left(\beta_{1}, \boldsymbol{\gamma}_{1}\right)^{(r+1)}, \boldsymbol{\lambda}_{1}^{(r+1)},\left(\boldsymbol{\beta}_{2}, \boldsymbol{\gamma}_{2}\right)^{(r+1)}, \boldsymbol{\lambda}_{2}^{(r+1)}, \boldsymbol{b}^{(r)}, \boldsymbol{E}^{*(r+1)}, D_{o b s}\right)$, com valor inicial $\theta^{(r)}$;

- $\operatorname{Gerar} \boldsymbol{\alpha}^{(r+1)} \sim \pi\left(\boldsymbol{\alpha}^{(r+1)} \mid \boldsymbol{E}^{*(r+1)}, D_{o b s}\right)$, com valor inicial $\boldsymbol{\alpha}^{(r)}$;

- Gerar $B^{(r+1)} \sim \pi\left(B^{(r+1)} \mid \boldsymbol{b}^{(r)}\right)$ em que $(B \mid \boldsymbol{b}) \sim$ Gama Inversa $\left(\frac{L}{2}+\kappa_{B} ; \frac{\sum_{l=1}^{L} b_{l}^{2}}{2}+\right.$ $\left.\varsigma_{B}\right)$ com $L$ sendo o número de grupos, $b_{l}$ o efeito aleatório $l=1, \cdots, L$ e $\left(\kappa_{B}, \varsigma_{B}\right)$ os hiperparâmetros da distribuição a priori para $B$, relembrando que $\pi(B)=$ Gama Inversa $\left(\kappa_{B}, \varsigma_{B}\right)$, e finalmente.

- Gerar $\boldsymbol{b}^{(r+1)} \sim \pi\left(\boldsymbol{b}^{(r+1)} \mid\left(\beta_{0}, \boldsymbol{\gamma}_{0}\right)^{(r+1)}, \boldsymbol{\lambda}_{0}^{(r+1)},\left(\beta_{1}, \boldsymbol{\gamma}_{1}\right)^{(r+1)}, \boldsymbol{\lambda}_{1}^{(r+1)},\left(\boldsymbol{\beta}_{2}, \boldsymbol{\gamma}_{2}\right)^{(r+1)}, \boldsymbol{\lambda}_{2}^{(r+1)}\right.$, $\left.\theta^{(r+1)}, B^{(r+1)}, D_{o b s}\right)$, com valor inicial $\boldsymbol{b}^{(r)}$.

Observação. Quando se trata do modelo sem efeitos aleatórios, as distribuições a posteriori condicionais completas dos parâmetros não dependem de $\boldsymbol{b}$ nem de $B$. Portanto, neste passo são desconsideradas as gerações de valores para o vetor $\boldsymbol{b}$ e sua variância $B$.

Passo 2. Fazer $r=r+1$ e voltar ao passo 1 .

Este algoritmo é repetido até a cadeia atingir uma distribuição estacionária e gerar a amostra a posteriori desejada.

Após a implementação do programa, seguindo este algoritmo e com as configurações dos hiperparâmetros conforme descrito na Seção 3.1.2, é feito um estudo de simulação com o objetivo de analisar o desempenho do modelo segundo alguns aspectos importantes na modelagem. Os detalhes do processo de simulação, assim como os resultados são discutidos na sequência.

\subsection{Estudo de simulação}

Um estudo de simulação foi realizado segundo a cópula de Clayton com os seguintes objetivos:

- validar o programa para a inferência bayesiana;

- avaliar o desempenho do método de estimação observando o comportamento das densidades a posteriori de cada parâmetro em relação aos verdadeiros valores 
usados como parâmetros na geração dos conjuntos de dados (apresentados na Tabela 4.1);

- examinar a robustez da estimação. Quando os dados são gerados segundo uma distribuição diferente à distribuição exponencial, considera-se uma má especificação debido a que é usado o modelo exponencial por partes para modelar as funções de risco basal. A ideia é observar a influência das estimativas dos parâmetros das funções de risco basal nas estimativas dos demais parâmetros quando a distribuição weibull é usada na geração dos dados.

Para determinar os cenários finais de simulação, foram feitos os seguintes testes prévios:

- com uma réplica: gera-se um conjunto de dados de diferentes tamanhos $N=500$, 1000 e 3000. Usando a mesma semente foram testadas diferentes partições para as funções de risco basais, usando $\left(J_{0}, J_{1}, J_{2}\right)=(1,1,1)$ até $\left(J_{0}, J_{1}, J_{2}\right)=(6,6,6)$. Para cada parâmetro são observadas as funções densidade a posteriori, a evolução das cadeias de Markov através dos traços das sequências simuladas e a função de autocorrelação (ACF).

- com 20 réplicas: usando três partições para $\boldsymbol{\lambda}_{0}, \boldsymbol{\lambda}_{1}$ e $\boldsymbol{\lambda}_{2}$, i.e. $\left(J_{0}, J_{1}, J_{2}\right)=(3,3,3)$, valores que levaram a melhores resultados na análise anterior, foram calculadas para todos os parâmetros na modelagem: a média a posteriori, desvio padrão $a$ posteriori, viés entre a média a posteriori e o verdadeiro valor do parâmetro, raiz do erro quadrático médio e porcentagem de cobertura do intervalo de credibilidade de $95 \%$, obtido pelos percentis a posteriori de $2,5 \%$ e $97,5 \%$ de cada parâmetro.

Estes testes foram efetuados com cadeias MCMC de 5000 iterações com um período de aquecimento de 1000 e espaçamento de 10 (com o propósito de tirar o efeito dos valores inicias e as autocorrelações) em todos os parâmetros, levando em conta, além disso, diferentes porcentagens de censura $(0 \%, 25 \%, 50 \%$ e $75 \%)$ e valores para o parâmetro da cópula de Clayton $(\theta=(0,5 ; 1 ; 2))$. Foram considerados também os tempos de execução.

A partir de tais testes iniciais, para o estudo de simulação final, apresentado a seguir, foram consideradas as duas modelagens, com e sem efeito aleatório, nas quais foram usados 200 conjuntos de dados de tamanhos $N=500$ e $N=1000$ com os tempos $T_{D}, T_{E}, T_{G}$, covariáveis e censura sob os seguintes cenários:

1. três partições para as funções de risco basal $\boldsymbol{\lambda}_{0}, \boldsymbol{\lambda}_{1}$ e $\boldsymbol{\lambda}_{2}$ i.e, $\left(J_{0}, J_{1}, J_{2}\right)=(3,3,3)$;

2. três porcentagens de censura: $10 \%, 45 \%$ e $70 \%$. Relembre que isto é a porcentagem de indivíduos nos casos 3 e 4, respectivamente (Seção 3.1.1);

3. dois valores para o parâmetro da cópula, $\theta=1$ e $\theta=2$ indicando uma dependência positiva de $\tau=0,33$ e $\tau=0,5$, respectivamente. Para a modelagem sem efeito aleatório não foi usado o valor de $\theta=2$ devido ao comportamento similar ao de $\theta=1$, e finalmente; 
4. para o caso do modelo com efeito aleatório (modelo misto), analisamos 10 grupos desbalanceados $(L=10)$.

Nas situações anteriories, os tempos das amostras simuladas foram gerados seguindo uma distribuição exponencial levando em conta que a estimação das funções de risco basal é feita através da função exponencial por partes. No entanto, foram levados em conta outros dois cenários para o modelo geral (modelo misto), (1) com $N=1000$, $\theta=1$ e $10 \%$ de censura e (2) com $N=500, \theta=1$ e $45 \%$ de censura, ambos com tempos gerados segundo uma distribuição Weibull, com o objetivo de observar a influência das funções de risco basais na inferência dos outros parâmetros.

\subsubsection{Geração das amostras}

O processo de geração das amostras começa a partir das covariáveis. A variável basal $A$ é gerada a partir de uma distribuição Bernoulli com probabilidade 0,5 que indica se o indivíduo está ou não em tratamento. Gera-se, independentemente, duas covariáveis basais: uma contínua e outra dicotômica com distribuições Normal e Bernoulli, $X_{1} \sim$ $\mathrm{N}(0,1)$ e $X_{2} \sim \operatorname{Ber}(0,6)$, respectivamente.

Considerando-se estas variáveis e valores pré-fixados para $\boldsymbol{\alpha}$ como indicado na Tabela $4.1^{1}$, gera-se a variável indicadora da progressão $E$ a partir do modelo logito

$$
\operatorname{logit}(\operatorname{Pr}(\mathrm{E}=1 \mid \mathrm{A}, \boldsymbol{x}))=\log \left(\frac{\operatorname{Pr}(\mathrm{E}=1 \mid \mathrm{A}, \boldsymbol{x})}{1-\operatorname{Pr}(\mathrm{E}=1 \mid \mathrm{A}, \boldsymbol{x})}\right)=\alpha_{0}+\mathrm{A} \alpha_{1}+\boldsymbol{x}^{\top} \boldsymbol{\alpha}_{\mathbf{2}} .
$$

Assim, obtemos $E$ como $\operatorname{Ber}(p) \operatorname{com} p=\frac{\exp \left\{\alpha_{0}+\alpha_{1} A+\alpha_{2} X_{1}+\alpha_{3} X_{2}\right\}}{1+\exp \left\{\alpha_{0}+\alpha_{1} A+\alpha_{2} X_{1}+\alpha_{3} X_{2}\right\}}$.

Após isto, no modelo misto, geram-se os efeitos aleatórios como $L$ variáveis aleatórias com distribuição $\mathrm{N}(0,1)$, designadas aleatoriamente aos $L$ grupos. Todos os indivíduos em um mesmo grupo recebem o mesmo efeito aleatório.

Em seguida, são geradas duas covariáveis, $Z$ com distribuição Normal truncada em 0, se $E=1$ e $V$ com distribuição $\operatorname{Ber}(p), \operatorname{com} p=\frac{\exp \left\{-0,5+0,2 X_{1}+0,5 Z\right\}}{1+\exp \left\{-0,5+0,2 X_{1}+0,5 Z\right\}}$ dado que $E=1$ e $A=1$.

Posteriormente, obtém-se três tempos basais segundo a distribuição Exponencial ou Weibull com as seguintes especificações:

- $T_{D}$ : tempo até o óbito, sob o modelo $T_{D},(3.2)$ e assumindo $H_{0}(t)=t$;

- $T_{E}$ : tempo até a progressão, sob o modelo $T_{E},(3.4) \operatorname{com} H_{1}(t)=5 t$;

- $T_{G}$ : tempo até o óbito após progressão, sob o modelo $T_{G},(3.5)$ em que $H_{2}(t)=t$.

\footnotetext{
${ }^{1}$ Os valores dos parâmetros $\left(\boldsymbol{\alpha}, \beta_{0}, \gamma_{0}, \beta_{1}, \boldsymbol{\gamma}_{1}, \boldsymbol{\beta}_{2}, \gamma_{2}\right)$, correspondem com os mesmos reportados por Zhang et al. (2014). Para posteriores estudos de simulação estes valores podem ser mudados, segundo uma análise dos dados reais.
} 
Note que a geração destes tempos depende da variável $E$. Se $E=0$ gera-se $T_{D}$ e se $E=1$ gera-se $\left(T_{E}, T_{G}\right)$ conjuntamente. Para gerar $\left(T_{E}, T_{G}\right)$ é usado o pacote CDVine versão 1.4 desenvolvido por Schepsmeier et al. $(2015)^{2}$, com o qual incorpora-se a dependência entre $T_{E}$ e $T_{G}$ através das cópulas. Os tempos $\left(T_{E}, T_{G}\right)$ foram gerados com a versão rotacionada em 180 graus para obter as correspondentes cópulas de sobrevivência.

Para a geração destes três tempos deve-se atentar ao fato de que a função de distribuição Weibull sob a parametrização em R é dada por

$$
F(x)=1-\exp \left\{-\left(\frac{x}{k}\right)^{a}\right\}, \quad x>0,
$$

sendo $a$ e $k$ os parâmetros de forma e escala, respectivamente. Deste modo, a função de risco é dada por $h(x)=a k^{-a} x^{a-1}$ e portanto, os tempos Weibull são gerados assumindo $a$ fixo e $k=\exp \left\{-\frac{1}{a}\left(\log \left(h_{0}(t)\right)+\boldsymbol{x}^{\top} \boldsymbol{\beta}+b\right)\right\}$, segundo seja o preditor linear de efeitos fixos, $K_{D}=A \beta_{0}+\gamma_{01} X_{1}+\gamma_{02} X_{2}, K_{E}=A \beta_{1}+\gamma_{11} X_{1}+\gamma_{12} X_{2}$, e $K_{G}=A \beta_{21}+V(1-A) \beta_{22}+$ $\gamma_{21} X_{1}+\gamma_{22} X_{2}+\gamma_{23} Z$, para os modelos $T_{D}, T_{E}$ e $T_{G}$, respectivamente. Cabe ressaltar que para amostras sem efeito aleatório, no parâmetro de escala é substraído o vetor $\boldsymbol{b}$, ou seja, $k=\exp \left\{-\frac{1}{a}\left(\log \left(h_{0}(t)\right)+\boldsymbol{x}^{\top} \boldsymbol{\beta}\right)\right\}$. Assim, para gerar tempos exponenciais é assumido $a=1$ e para gerar os tempos weibull é assumido $a=2$. Os valores dos parâmetros adotados para o cálculo dos preditores lineares dos efeitos fixos $K_{D}, K_{E}$, e $K_{G}$ são resumidos na Tabela 4.1.

Tabela 4.1: Valores dos parâmetros utilizados na geração dos conjuntos de dados.

\begin{tabular}{l|c|l}
\hline \hline Modelo Logito & $\boldsymbol{\alpha}$ & $\alpha_{0}=1,6, \alpha_{1}=-1,8, \alpha_{2}=1, \alpha_{3}=0,1$ \\
\hline Modelo $T_{D}$ & $\left(\beta_{0}, \gamma_{0}\right)$ & $\beta_{0}=-1, \gamma_{01}=1, \gamma_{02}=0.2$ \\
\hline Modelo $T_{E}$ & $\left(\beta_{1}, \gamma_{1}\right)$ & $\beta_{1}=-0,5, \gamma_{11}=1, \gamma_{12}=0$ \\
\hline Modelo $T_{G}$ & $\left(\boldsymbol{\beta}_{\mathbf{2}}, \gamma_{2}\right)$ & $\beta_{21}=-0,3, \beta_{22}=-0,5, \gamma_{21}=-0,5, \gamma_{22}=0,5, \gamma_{23}=0,4$ \\
\hline \hline
\end{tabular}

\section{Adição da censura}

Na estrutura de riscos semicompetitivos, a censura está associada a duas situações, correspondentes aos casos 3 e 4 como descritos na Seção 3.1.1. Neste trabalho optou-se por submeter os tempos gerados anteriormente, $T_{D}$ e $\left(T_{E}, T_{G}\right)$ a dois tipos de censura à direita como segue:

\footnotetext{
${ }^{2}$ Detalhes e uso do pacote CDVine podem ser consultados em Brechmann e Schepsmeier (2013).
} 
(i). Censura Tipo I, indicando um periodo máximo de acompanhamento de $\eta$, isto é, se os tempos gerados $T_{D}$ e $T_{E}$ são maiores que $\eta$, tem-se o caso $4(d=0, v=0)$. O caso $3(d=0, v=1)$ será observado na situação em que $T_{E}<\eta \leq T_{E}+T_{G}$. Nas amostras geradas, utilizou-se $\eta=7$ sob a cópula de Clayton.

(ii). Censura aleatória, gerada quando a proporção de censura denotada por $p c$ não for atingida pela censura tipo I. Nesta fase um tempo de censura $C$ é gerado como $C \sim \operatorname{Unif}(0, \eta)$. Uma observação a ser censurada é escolhida ao acaso no grupo dos não censurados com tempos $T_{D}$ e $T_{E}+T_{G}$ maiores do que $C$. Esta observação é classificada como caso 4 se $T_{D}>C$ ou se $T_{E}+T_{G}>C$ e corresponderá ao caso 3 se $T_{E}<C \leq T_{E}+T_{G}$.

Assim, em decorrência das censuras, pode-se obter as quatro situações nos dados simulados, já mencionadas:

- Caso 1: Se $T_{D}<\eta$, o óbito é observado e assim para o $i$-ésimo indivíduo do grupo $l$ no conjunto de dados tem-se,

$$
\begin{aligned}
D_{i l}= & \left\{Y_{i l}=T_{D}, Y_{E i l}=0, Y_{G i l}=0, A_{i l}, V_{i l}=\mathrm{NA}, \boldsymbol{X}_{i}=\left(X 1_{i l}, X 2_{i l}\right), Z_{i l}=\mathrm{NA},\right. \\
& \left.d_{i l}=0, v_{i l}=1\right\} .
\end{aligned}
$$

- Caso 2: Se $T_{E}+T_{G}<\eta$, então considera-se que os dois eventos de interesse foram observados e obtém-se para o $i$-ésimo individuo do grupo $l$,

$$
\begin{aligned}
D_{i l}= & \left\{Y_{i l}=0, Y_{E i l}=T_{E}, Y_{G i l}=T_{G}, A_{i l}, V_{i l}, \boldsymbol{X}_{i l}=\left(X 1_{i l}, X 2_{i l}\right), Z_{i l}=Z_{i l}, d_{i l}=1,\right. \\
& \left.v_{i l}=1\right\} .
\end{aligned}
$$

- Caso 3: Comparando o tempo de censura gerado $C$, se $T_{E}<C<T_{E}+T_{G}$, então é observado o tempo até a recaída. O tempo até o óbito é censurado, obtendo o registro

$$
\begin{aligned}
D_{i l}= & \left\{Y_{i l}=0, Y_{E i l}=T_{E}, Y_{G i l}=C, A_{i l}, V_{i l}, \boldsymbol{X}_{i l}=\left(X 1_{i l}, X 2_{i l}\right), Z_{i l}=Z_{i l}, d_{i l}=1,\right. \\
& \left.v_{i l}=0\right\} .
\end{aligned}
$$

- Caso 4: Comparando o tempo de censura gerado $C$, se $C<T_{E}$, então obtém-se a situação em que não se apresenta nenhum dos eventos de interesse. Assim,

$$
\begin{aligned}
D_{i l}= & \left\{Y_{i l}=C, Y_{E i l}=0, Y_{G i l}=0, A_{i l}, V_{i l}=\mathrm{NA}, \boldsymbol{X}_{i l}=\left(X 1_{i l}, X 2_{i l}\right), Z_{i l}=\mathrm{NA},\right. \\
& \left.d_{i l}=0, v_{i l}=0\right\} .
\end{aligned}
$$

Com dados simulados desta forma sob os diferentes cenários já mencionados, foram obtidos os resultados descritos a seguir. Todas as tabelas e gráficos produto das simulações encontram-se no Apêndice C. 


\subsubsection{Resultados para a cópula de Clayton}

Para cada conjunto de dados gerado foram usadas cadeias de 10000 iterações de Gibbs com um período de aquecimento de 2000 e emagrecimento de 10. Em todas as situações, considerou-se três partições para as funções de risco basal $\left(J_{0}, J_{1}, J_{2}\right)=(3,3,3)$. As tabelas dos resultados contêm, nas primeiras entradas, os parâmetros a ser estimados com seus verdadeiros valores. Para cada um deles são resumidos: a média das 200 réplicas das médias a posteriori (Est), o média dos desvios padrões a posteriori (SD), a média das raízes quadradas do erro quadrático médio (RMSE) e a probabilidade de cobertura do intervalo de alta densidade a posteriori (HPD) de $95 \%$.

Com estas especificações os resultados são apresentados da seguinte forma:

1. Resultados para o modelo misto com 10 grupos desbalanceados (em termos da aplicação, da IRC, os grupos fazem referência às cidades onde foi tratado o indivíduo) e com tempos $T_{D}, T_{E}$ e $T_{G}$ gerados segundo a distribuição exponencial. As Tabelas C.1 a C.6 correspondem aos resultados, sendo que as primeiras três correspondem ao valor para o parâmetro da cópula $\theta=1$ com as respectivas proporções de censura, $10 \%, 45 \%$ e $70 \%$. As últimas três tabelas correspondem ao valor de $\theta=2$ com as mesmas proporções de censura.

2. Resultados para o modelo geral com tempos gerados segundo a distribuição Weibull nas Tabelas C.7 e C.8.

3. Resultados para o modelo sem efeito aleatório com tempos gerados segundo a distribuição exponencial nas Tabelas C.9, C.10 e C.11, correspondentes a $\theta=1$ com as respectivas proporções de censura, 10\%, $45 \%$ e $70 \%$.

4. As Figuras C.1 a C.11 representam uma comparação das médias e desvios padrão $a$ posteriori dos parâmetros (excepto os correspondentes às funções de risco basal) entre as modelagens mistas $\operatorname{com} \theta=1, \theta=2$ (primeira e segunda coluna de figuras) e a modelagem sem efeito aleatório (fixa) $\operatorname{com} \theta=1$ (terceira coluna). De maneira geral, a escala dos mesmos foi fixada em 0,5 acima e abaixo do valor real do parâmetro (representado pela linha horizontal).

\section{Comentários: Modelagem com efeito aleatório - mista}

Observa-se, conforme Tabelas C.1, C.2 e C.3, que em geral se obteve o comportamento esperado segundo as proporções de censura especificadas.

As melhores estimativas foram para $10 \%$ de censura, em que particularmente as médias a posteriori (Est) são próximas dos verdadeiros valores com os quais foram geradas as amostras. Os desvios padrões (SD) e o RMSE são coerentes, o que dá indícios que a incerteza nas estimativas são explicadas adequadamente. Estas estimativas melhoram quando o tamanho das amostras aumenta. Embora, ao observar a porcentagem de cobertura ela é maior para $\mathrm{N}=1000$ que para $\mathrm{N}=500$ em $65 \%$ dos parâmetros estimados. No entanto, observa-se que estas porcentagens de cobertura ficaram acima de $90 \%$ para os 26 parâmetros. Aumentando o número de réplicas, por exemplo 500 réplicas, como 
em Zhang et al. (2014) possivelmente estas porcentagens melhorariam.

Pelas Tabelas C.2 e C.3, nota-se um aumento do viés proporcional ao aumento da censura, que parece ser mais acentuado para os parâmetros associados às funções de risco basais $\left(\boldsymbol{\lambda}_{0}, \boldsymbol{\lambda}_{1}, \boldsymbol{\lambda}_{2}\right)$. Porém, as porcentagens de cobertura continuam sendo relativamente altas para a maioria dos parâmetros tanto para $\mathrm{N}=500$ quanto para $\mathrm{N}=1000$.

Ao analisar os resultados das Tabelas C.4, C.5 e C.6, nas quais $\theta=2$, interpretações similares às tabelas anteriores podem ser feitas, indicando que na inferência dos outros parâmetros não se apresentam diferenças pronunciadas quando se muda o nível de dependência entre os tempos até os eventos intermediário e final. Isto dá indícios de que a modelagem é apropriada para diferentes níveis de dependência.

Nas Tabelas C.7 e C.8 são apresentados os resultados da simulação quando os conjuntos de dados foram gerados segundo uma distribução Weibull para os tempos $T_{D}$, $T_{E}$ y $T_{G}$. Nestas situações, as estimatições das funções de risco basal foram as mais afastadas dos verdadeiros parâmetros e com isto as inferências dos outros parâmetros são afetadas negativamente, exceto para o modelo logito em que as porcentagens de cobertura ficaram acima de $93 \%$.

Em relação à variância do efeito aleatório, observa-se boas estimativas, inclusive quando a porcentagem de censura é variada.

\section{Comentários: Modelagem sem efeito aleatório - fixa}

A inferência no modelo sem efeito aleatório não possui diferenças marcantes em relação ao modelo misto. Podemos ver isto nas Tabelas C.9, C.10 e C.11. O aumento no tamanho da amostra implica em uma diminuição na dispersão das estimativas, as quais ficam mais próximas do verdadero valor dos parâmetros à medida em que se tem menor proporção de censura. Além disso, obtém-se altas porcentagens de cobertura sendo iguais ou superiores a 95\% em 200 réplicas quando há 10\% de censura, acima de 91\% de cobertura com $45 \%$ de censura para a maioria dos parâmetros (exceto em seis deles) e acima de $88 \%$ de cobertura com $70 \%$ de censura, sem discriminar por tamanho de amostra em todas as situações.

Em relação ao parâmetro da cópula, que pode não ser simples de estimar (Lakhal et al., 2008), observa-se estimativas adequadas e altas porcentagens de cobertura até com $45 \%$ de censura para amostras de tamanho 500 e 1000. Já com censura superior a 45\%, a estimativa torna-se sobrestimada, apesar da baixa dispersão.

\section{Comentários: Figuras}

Neste trabalho são apresentados os resultados gráficos das simulações para comparar mais facilmente as estimativas dos parâmetros e a dispersão, separados segundo a variável ao qual está associado. Além disso, são mostradas as estimativas para as modelagens fixa e mista para efeito de comparação. 
- Modelo $T_{D}$ : Coeficientes associados à modelagem do óbito sem progressão, $\beta_{0}, \gamma_{01}$ e $\gamma_{02}$ apresentadas nas Figuras C.1 e C.2.

Nesta componente, o coeficiente mais afetado pela censura foi $\beta_{0}$ associado ao efeito do tratamento, sendo subestimado quando aumenta a censura. Os coeficientes $\gamma_{01}$ e $\gamma_{02}$ associados às covariáveis $X_{1} \sim \mathrm{N}(0,1)$ e $X_{2} \sim \operatorname{Ber}(0,6)$, respectivamente, foram sobrestimados, em maior proporção para $\gamma_{01}$ do que para $\gamma_{02}$; em compensação, $\gamma_{01}$ apresenta menor dispersão que $\gamma_{02}$.

- Modelo $T_{E}$ : Coeficientes associados à modelagem da progressão, $\beta_{1}, \gamma_{11}$ e $\gamma_{12}$ mostrados nas Figuras C.3 e C.4.

As estimativas nesta componente dão indícios de um bom ajuste dado que as estimativas dos três parâmetros foram pouco afetados pela censura, além de apresentar pequenas dispersões, principalmente para $\gamma_{11}$ (covariável $x_{1}$ ). Este coeficiente apresenta uma leve sobrestimação na modelagem mista.

- Modelo $T_{G}$ : Coeficientes associados à modelagem do óbito após progressão, $\beta_{21}$, $\beta_{22}, \gamma_{21}, \gamma_{22}$ e $\gamma_{23}$ conforme as Figuras C.5, C.6 e C.7.

Relembre que $\beta_{21}$ e $\beta_{22}$, estão associadas ao tratamento $A$ e à mudança de tratamento $V(1-A)$. Aqui as melhores estimativas foram observadas no modelo misto (colunas 1 e 2) que foram menos afetadas pela censura do que o modelo fixo (coluna 3). Em ambos os casos nota-se uma mudança significativa na dispersão quando a censura passa de $45 \%$ a $70 \%$. A respeito dos coeficientes $\gamma_{21}, \gamma_{22}$ e $\gamma_{23}$, associados respectivamente às covariáveis $x_{1}, x_{2}$ e $z$ ( $Z$ medida após a progressão), observa-se que as melhores estimativas foram para $\gamma_{21}$; para $\gamma_{22}$ são sobrestimadas com o aumento da censura, e para $\gamma_{23}$ elas são subestimadas.

- Modelo logito: Coeficientes associados à modelagem da probabilidade da ocorrência da progressão, $\alpha_{0}, \alpha_{1}, \alpha_{2}$ e $\alpha_{3}$ conforme as Figuras C.8 e C.9.

Comparando com as outras componentes (modelos $T_{D}, T_{E}$ e $T_{G}$ ), as estimativas aqui apresentam maiores dispersões. As estimativas de $\alpha_{3}$, correspondente ao efeito de $x_{2}$, não parecem ser afetadas pela censura. Já o intercepto $\alpha_{0}$ e o coeficiente da covariável $x_{1}, \alpha_{2}$ são sobrestimados proporcionalmente com o aumento da censura e o coeficiente $\alpha_{1}$ associado com o tratamento $A$, subestimado.

- Parâmetro da cópula $\theta$ : mostrado na Figura C.10.

Os gráficos destas figuras mostram o acentuado efeito da censura na estimativa de $\theta$, apresentando uma maior dispersão quando a dependência é maior, ou seja, quando $\theta=2$ (indicando $\tau=0,5$, figura do meio). Quando a dependência entre os tempos até a progressão e óbito é menor, não se observam diferenças entre a modelagem mista (figura à esquerda) e fixa (figura à direita).

- Variância do efeito aleatório B, conforme Figura C.11.

Em geral, é possível concluir que a censura não tem efeito na estimativa deste parâmetro, tampouco o tamanho da amostra. O que se observa a primera vista é uma dispersão ampla. 
Observa-se que em geral não há grandes diferenças nas inferências quando se usa a modelagem mista ou fixa. Além disso, em todos os parâmetros, exceto na variância do efeito aleatório $B$, a dispersão é menor com amostras maiores, como era de se esperar.

As componentes da modelagem com estimativas mais próximas aos verdadeiros valores e menores dispersões foram para os parâmetros associados aos modelos $T_{E}$ e $T_{G}$. 


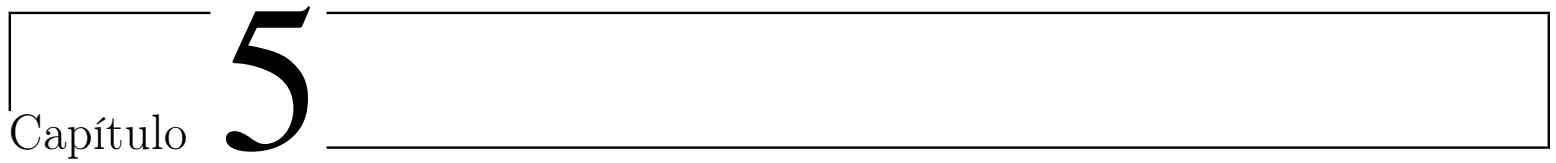

\section{Aplicação}

Neste capítulo é apresentada uma breve descrição das medidas de ajuste que serão usadas nesta tese como medidas de comparação de modelos e que são o suporte para determinar o ajuste mais adequado a um conjunto dados. Na sequência, é aplicada a modelagem proposta segundo a cópula de Clayton a dois conjuntos de dados. O primeiro deles corresponde a pacientes com leucemia que receberam transplante de medula óssea (TMO). Os dados se encontram disponíveis com o nome ebmt 4 no pacote mstate em R. Para estes dados, é ajustada a modelagem sem efeito aleatório (modelo fixo). O segundo conjunto de dados, como discutido no Capítulo 1, corresponde ao estudo de insuficiência renal crônica (IRC), que pretende avaliar a eficácia do tratamento e o efeito das covariáveis na sobrevivência dos pacientes observados. Para os dados de IRC, além de uma análise descritiva, são aplicados os modelos propostos com e sem efeitos aleatórios (modelagem mista e fixa).

\subsection{Comparação de modelos}

Considerando que para os modelos propostos deste trabalho podem ser escolhidas diferentes partições para as funções de risco basal, há interesse em comparar o ajuste de vários modelos aos dados quando são assumidas diferentes partições para $\left(J_{0}, J_{1}, J_{2}\right)$.

As medidas de comparação de modelos consideradas são:

- Estatística do logaritmo da pseudoverossimilhança marginal (LPML)

Esta medida é calculada com base nas ordenadas preditivas condicionais (CPO), proposta por Geisser (1993), e é definida como LPML $=\sum_{i, l} \log \left(\mathrm{CPO}_{i l}\right)$, em que valores maiores de LPML indicam melhor ajuste. Para o $i$-ésimo indivíduo do l-ésimo grupo, a estatística $\mathrm{CPO}_{i l}$ é definida como

$$
\mathrm{CPO}_{i l}=\mathrm{L}\left(D_{i l} \mid D^{(-i l)}\right)=\int \mathrm{L}_{i l}\left(\boldsymbol{\xi}, B \mid D_{i l}\right) \pi\left(\boldsymbol{\xi}, B \mid D^{(-i l)}\right) d B d \boldsymbol{\xi},
$$

em que $D^{(-i l)}$ denota os dados sem a observação do $i$-ésimo indivíduo no $l$ - 
ésimo grupo, $\mathrm{L}_{i l}\left(\boldsymbol{\xi}, B \mid D_{i l}\right)$ denota a função verossimilhança para o $i$-ésimo indivíduo no l-ésimo grupo, corresponde ao termo no produto da expressão (3.11), e $\pi\left(\boldsymbol{\xi}, B \mid D^{(-i l)}\right)$ é a função densidade a posteriori dado os dados $D^{(-i l)}$. Segundo Lee et al. (2016), intuitivamente o $\mathrm{CPO}_{i l}$ é a probabilidade a posteriori do resultado observado para o $i$-ésimo indivíduo no $l$-ésimo grupo com base no modelo ajustado aos dados que excluem esse indivíduo especificamente. Portanto, grandes valores do $\mathrm{CPO}_{i l}$ atribuem altas probabilidades aos dados observados, indicando um melhor ajuste.

Em muitos modelos de sobrevivência, não é possível obter uma expressão analítica para esta medida. No entanto, um estimador Monte Carlo de $\mathrm{CPO}_{i l}$ pode ser obtido usando uma amostra MCMC da distribuição a posteriori $\pi\left(\boldsymbol{\xi}, B \mid D_{o b s}\right)$. De acordo com Chen et al. (2000) (Cap. 10), uma aproximação da $\mathrm{CPO}_{i l}$ é dada por

$$
\widehat{\mathrm{CPO}}_{i l}=\left(\frac{1}{Q} \sum_{q=1}^{Q} \frac{1}{\mathrm{~L}_{i l}\left(\boldsymbol{\xi}_{q}, B_{q} \mid D_{i l}\right)}\right)^{-1},
$$

$\operatorname{com} q=1, \cdots, Q$ amostras MCMC da distribuição a posteriori de $(\boldsymbol{\xi}, B)$. Outra medida que se encontra na literatura baseada no CPO é

$$
\mathrm{ALPML}=\frac{L P M L}{N}
$$

com $N$ sendo o tamanho da amostra, i.e o número de observações. Esta estatística pode ser interpretada como a pseudoverossimilhança marginal relativa (Ibrahim et al., 2000).

- critério de informação bayesiano (BIC)

Conforme Sahu e Dey (2000), este critério é definido como

$$
\mathrm{BIC}=-2(\text { função log-verossimilhança calculada no EMV })+p \log (N),
$$

em que $N$ é o tamanho da amostra e $p$ é o número estimado de parâmetros. Como estes autores comentam, com uma implementação via MCMC não é possível maximizar a log-verossimilhança requerida para calcular o BIC. No entanto, o valor médio da função de verossimilhança avaliada a cada iteração MCMC pode ser usada como uma aproximação. Desta forma, o critério pode ser definido como

$$
\widehat{B I C}=-\frac{2}{Q} \sum_{q=1}^{Q} \sum_{i, l} \log \left(\mathrm{L}_{i l}\left(\boldsymbol{\xi}_{q}, B_{q} \mid D_{i l}\right)\right)+p \log N
$$

em que $\left(\boldsymbol{\xi}_{q}, B_{q}\right)$ corresponde à uma amostra da distribuição a posteriori obtida na $q$-ésima iteração no algoritmo MCMC e $p$ é o número de parâmetros associados ao modelo. 
No caso do BIC, valores menores indicam um melhor ajuste.

- Criterio de informação de desviância (DIC)

Como indicam Lee et al. (2016), esta estatística é frequentemente escolhida para a comparação de modelos no paradigma bayesiano. De acordo com Spiegelhalter et al. (2002) e assumindo $\boldsymbol{\vartheta}=(\boldsymbol{\xi}, B)$, o DIC é uma medida definida como DIC $=$ $\operatorname{Dev}(\overline{\boldsymbol{\vartheta}})+2 p_{D}=\overline{\operatorname{Dev}(\boldsymbol{\vartheta})}+p_{D}$, com o termo de penalidade dado por $p_{D}=\overline{\operatorname{Dev}(\boldsymbol{\vartheta})}-$ $\operatorname{Dev}(\overline{\boldsymbol{\vartheta}})$ que corresponde ao número efetivo de parâmetros. Aqui a desviância é definida como $\operatorname{Dev}(\boldsymbol{\vartheta})=\sum_{i, l}-2 \log \left(\mathrm{L}\left(\boldsymbol{\xi}, B \mid D_{i l}\right)\right)$, sendo $\mathrm{L}\left(\boldsymbol{\xi}, B \mid D_{i l}\right)$ o termo no produto da expressão (3.11). Já as quantidades $\overline{\boldsymbol{\vartheta}}$ e $\overline{\operatorname{Dev}(\boldsymbol{\vartheta})}=\mathbb{E}\left(\operatorname{Dev}\left(\boldsymbol{\xi}, B \mid D_{\text {obs }}\right)\right)$ denotam a média a posteriori de $\boldsymbol{\vartheta}$ e $\operatorname{Dev}(\boldsymbol{\vartheta})$, respectivamente, que podem ser estimadas por $\overline{\boldsymbol{\vartheta}}=\frac{1}{Q} \sum_{q=1}^{Q} \boldsymbol{\vartheta}_{q}$ e $\overline{\operatorname{Dev}(\boldsymbol{\vartheta})}=\frac{1}{Q} \sum_{q=1}^{Q} \operatorname{Dev}\left(\boldsymbol{\vartheta}_{q}\right)$, em que $\boldsymbol{\vartheta}_{1}, \cdots, \boldsymbol{\vartheta}_{Q}$ são amostras da distribuição a posteriori (Spiegelhalter et al. (2002), de Castro et al. $(2015))$.

Pequenos valores de DIC indicam os melhores ajustes.

Além das medidas de ajuste descritas para a escolha do modelo mais apropriado, neste trabalho também são usados: gráficos da sequência de valores simulados (traços) e gráficos da função de autocorrelação (ACF) da cadeia de Markov fornecida pela distribuição a posteriori.

Como indicam Lesaffre e Lawson (2012), para avaliar a convergência das cadeias de Markov é mais recomendável besear-se em critérios descritivos do que em testes de hipóteses, pois os desenvolvimentos teóricos têm gerado um impacto limitado na prática. Assim, optou-se por usar aproximações gráficas através da inspeção das sequências dos valores simulados e autocorrelação para inferir se a convergência foi atingida. Cabe destacar que os gráficos das autocorrelações não determinam exclusivamente a convergência, pois valores altos não implicam ausência de convergência e sim mistura lenta. Detalhes de avaliação das cadeias de Markov podem ser consultados, por exemplo, em Lesaffre e Lawson (2012) Seção 7.2, Ntzoufras (2009) Seção 2.2.

\subsection{Transplante de medula óssea (TMO)}

O conjunto de dados analisado nesta Seção provem da European Society for Blood and Marrow Transplantation (EBMT) e corresponde a 2279 pacientes transplantados entre 1985 e 1998. Na literatura, vários autores já usaram estes dados para ilustrar outras modelagens multiestado como, por exemplo, Fiocco et al. (2008), de Wreede et al. (2011) e de Castro et al. (2015).

No conjunto de dados de TMO foram registrados vários eventos. No entanto, para esta aplicação só foram considerados: o tempo em dias desde o transplante até a recaída, 
como evento intermediário, e o tempo em dias desde o transplante até o óbito, como evento terminal. Os fatores prognósticos, assim como o número de observações em cada caso, são apresentados na Tabela 5.1.

Tabela 5.1: Descrição das covariáveis e eventos de interesse nos dados de TMO.

\begin{tabular}{llcc}
\hline \hline & Categorias & $n$ & $\%$ \\
\hline Coariáveis & & 634 & 27,82 \\
\hline Ano de transplante & 1 1985 - 1989 & 896 & 39,31 \\
& $21990-1994$ & 749 & 32,86 \\
& $31995-1998$ & & \\
& & 551 & 24,18 \\
Idade do paciente & $1 \leq 20$ anos & 1213 & 53,22 \\
no transplante & $220-40$ anos & 515 & 22,60 \\
& $3>40$ anos & & \\
Profilaxia & & 1730 & 75,91 \\
& 0 Não & 549 & 24,09 \\
Compatibilidade sexo & 0 Sem incompatibilidade & 1734 & 76,09 \\
doador-receptor & 1 Incompatibilidade & 545 & 23,91 \\
& & & \\
\hline Eventos de interesse & & & \\
\hline Caso & 1 Óbito & 533 & 23,39 \\
& 2 Óbito após recaída & 305 & 13,38 \\
& 3 Recaída & 65 & 2,85 \\
& 4 Censura & 1376 & 60,38 \\
\hline \hline
\end{tabular}

Conforme Xu et al. (2010), uma forma intuitiva de identificar a força da dependência entre os eventos de interesse, recaída e óbito, é através da proporção de pacientes que apresentaram óbito após recaída entre todos os pacientes que apresentaram recaída. Da Tabela 5.1, pode se observar que tal proporção é 305/370 = 82,43\%. Espera-se com isto encontrar um grau de dependência moderado entre os eventos. Além disso, o gráfico de dispersão do tempo até recaída e o tempo até óbito após recaída apresentado na Figura 5.1 indica uma possível dependencia positiva.

\subsubsection{Ajuste da modelagem proposta}

Para os dados de TMO foi ajustado a modelo sem efeito aleatório (modelo fixo) com diferentes partições para as funções de risco basal e usando a cópula de Clayton. Para todos os modelos fixos ajustados foram adotados como hiperparâmetros para as distribuições a priori os sugeridos na literatura (Zhang et al. (2014), Ibrahim et al. (2000)). Assim, $\boldsymbol{\alpha} \sim \mathrm{N}_{p_{a}}\left(\mathbf{0}, \boldsymbol{\Sigma}_{a}\right)$ com $\boldsymbol{\Sigma}_{a}=1000 \boldsymbol{I}^{-1} p_{a}$ sendo $\boldsymbol{I}^{-1} p_{a}$ a matriz identidade inversa de dimensão $p_{a}=7$. Similarmente, $\left(\beta_{0}, \boldsymbol{\gamma}_{0}^{\top}\right)^{\top} \sim \mathrm{N}_{p_{0}}\left(\mathbf{0}, \boldsymbol{\Sigma}_{0}\right),\left(\beta_{1}, \boldsymbol{\gamma}_{1}^{\top}\right) \sim \mathrm{N}_{p_{1}}\left(\mathbf{0}, \boldsymbol{\Sigma}_{1}\right)$ e $\left(\boldsymbol{\beta}_{2}^{\top}, \boldsymbol{\gamma}_{2}^{\top}\right) \sim \mathrm{N}_{p_{2}}\left(\mathbf{0}, \boldsymbol{\Sigma}_{2}\right)$ com $p_{0}=p_{1}=p_{2}=6$ (número de covariáveis). Para $\lambda_{k j}$ são 


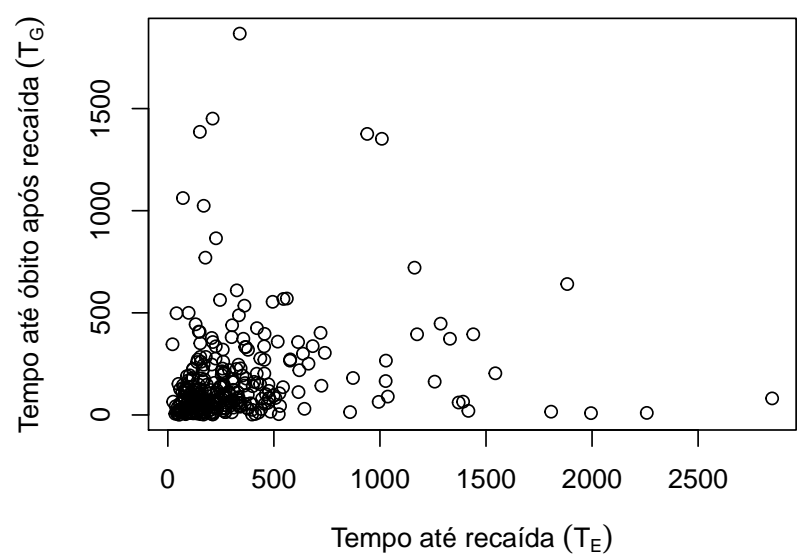

Figura 5.1: Diagrama de dispersão dos tempos até a ocorrência dos eventos de interesse, recaída e óbito, dos dados de TMO. Incluí sómente os indivíduos do caso 2.

assumidos $\lambda_{k j} \sim \operatorname{Gama}(0,01 ; 0,01)$ para $j=1, \cdots, J_{k}$ e $k=0,1,2$. Finalmente, para $\theta$ tem-se $\theta \sim \operatorname{GamaInv}(0,01 ; 0,01)$.

Para todos os modelos ajustados, foram usadas 10000 iterações de Gibbs, obtendo amostras de tamanho 800 após um aquecimento de 2000 e espaçamento de 10.

O resultado das medidas de comparação de modelos para alguns desses ajustes apresentam-se na Tabela 5.2.

Tabela 5.2: Valores LPML, BIC DIC para o modelo fixo sob a cópula de Clayton ajustados aos dados de TMO.

\begin{tabular}{cccccc}
\hline \hline \multicolumn{3}{c}{ Partições } & \multicolumn{3}{c}{ Modelo fixo } \\
\hline$J_{0}$ & $J_{1}$ & $J_{2}$ & LPML & BIC & DIC \\
\hline & & & & & \\
1 & 1 & 1 & $-11425,80$ & 23008,50 & 22803,61 \\
2 & 2 & 2 & $-10788,22$ & 21766,56 & 21542,44 \\
3 & 3 & 3 & $-10573,30$ & 21360,84 & 21114,43 \\
4 & 4 & 4 & $-10469,30$ & 21177,21 & 20905,85 \\
5 & 5 & 5 & $-10399,57$ & 21058,99 & 20765,86 \\
10 & 10 & 10 & $-10300,54$ & $\mathbf{2 0 9 6 0 , 3 3}$ & 20556,59 \\
15 & 15 & 15 & $\mathbf{- 1 0 2 7 8 , 9 0}$ & 21016,98 & $\mathbf{2 0 4 9 7 , 2 2}$ \\
2 & 12 & 5 & $-10838,88$ & 21962,62 & 21655,78 \\
5 & 10 & 8 & $-10744,54$ & 21802,52 & 21449,11 \\
& & & & & \\
\hline \hline
\end{tabular}

Conforme a Tabela 5.2 , o ajuste com as partições $\left(J_{0}, J_{1}, J_{2}\right)=(15,15,15)$ obteve o maior valor de LPML e o menor valor de DIC entre as nove combinações conside- 
radas para $J_{0}, J_{1}, J_{2}$ sob o modelo fixo. Por outro lado, o BIC detectou como melhor ajuste o modelo com as partições $\left(J_{0}, J_{1}, J_{2}\right)=(10,10,10)$. Considerando que os resultados de ambos ajustes foram relativamente próximos, no que segue são apresentados os resultados em que $\left(J_{0}, J_{1}, J_{2}\right)=(15,15,15)$.

\section{Resultados com o modelo fixo}

A Tabela 5.3 apresenta as estimativas a posteriori (média, desvio padrão (Sd.) e HPD de $95 \%)$ dos parâmetros segundo a modelagem com $\left(J_{0}, J_{1}, J_{2}\right)=(15,15,15)$.

No Apêndice D são apresentados os gráficos da função densidade a posteriori, os traços dos valores simulados e os gráficos da função de autocorrelação para todos os parâmetros estimados referentes aos resultados da Tabela 5.3. A Figura D.1 refere à inferência bayesiana para a componente do óbito $T_{D}$, a Figura D.2 para a componente da recaída $T_{E}$, a Figura D.3 para a componente óbito após recaída $T_{G}$ e a Figura D.3 para a probabilidade de recaída, componente logito. Aqui é apresentada à inferência Bayesiana para o parâmetro da cópula de Clayton conforme observa-se na Figura 5.2. De tais gráficos pode-se observar que em forma geral houve convergência para todos os parâmetros obtendo distribuições a posteriori simétricas com variâncias pequenas e sem grandes autocorrelações o que fornece indícios de convergência.
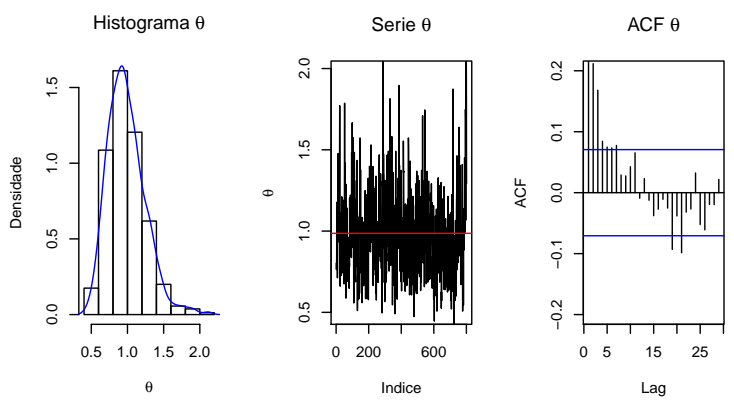

Figura 5.2: Função densidade a posteriori, traços e ACF para o parâmetro da cópula de Clayton do ajuste da modelagem fixa com $\left(J_{0}, J_{1}, J_{2}\right)=(15,15,15)$ aos dados de TMO.

Conforme a Tabela 5.3, o ano de transplante teve um efeito signigicativo sob as componentes $T_{D}, T_{E}$ e $T_{G}$, dado que os respectivos intervalos HPD não contém o zero. Da mesma forma, a idade do paciente resultou significativa na componente $T_{D}$. A compatibilidade do sexo doador-receptor também pode ser incluída na componente $T_{D}$ como relevante pois, segundo a Figura D.1, observa-se para $\gamma_{06}$ que a massa da distribuição a posteriori aparece concentrada à direita de 0 . A componente do óbito após recaída $\left(T_{G}\right)$ teve a idade como fator prognóstico significativo, além do ano de transplante.

A profilaxia só foi um fator representativo para a probabilidade de recaída (modelo logito). Embora o HPD de $95 \%$ contém o zero, ele fica na borda esquerda do intervalo e, conforme Figura D.4 para $\left(\alpha_{5}\right)$, a massa da distribuição a posteriori se concentra à 
Tabela 5.3: Ajuste modelagem fixa aos dados de $\operatorname{TMO} \operatorname{com}\left(J_{0}, J_{1}, J_{2}\right)=(15,15,15)$.

\begin{tabular}{|c|c|c|c|c|}
\hline \multicolumn{2}{|c|}{ Covariável / Parâmetro } & Média & Sd. & HPD 95\% \\
\hline \multicolumn{5}{|c|}{ Modelo $T_{D}$ (Óbito) } \\
\hline \multirow{3}{*}{ Ano do transplante } & 1985-1989 & & & \\
\hline & $1990-1994\left(\gamma_{01}\right)$ & $-0,509$ & 0,113 & $(-0,736 ;-0,294)$ \\
\hline & $1995-1998\left(\gamma_{02}\right)$ & $-0,537$ & 0,119 & $(-0,749 ;-0,299)$ \\
\hline \multirow{3}{*}{$\begin{array}{l}\text { Idade do paciente } \\
\text { no transplante }\end{array}$} & $\leq 20$ anos & & & \\
\hline & $(20-40]$ anos $\left(\gamma_{03}\right)$ & 0,614 & 0,122 & $(0,375 ; 0,847)$ \\
\hline & $>40 \operatorname{anos}\left(\gamma_{04}\right)$ & 0,947 & 0,140 & $(0,685 ; 1,218)$ \\
\hline \multirow[t]{2}{*}{ Profilaxia } & Não & & & \\
\hline & $\operatorname{Sim}\left(\gamma_{05}\right)$ & 0,085 & 0,099 & $(-0,103 ; 0,283)$ \\
\hline \multirow{3}{*}{$\begin{array}{l}\text { Compatibilidade sexo } \\
\text { doador-receptor }\end{array}$} & Não incompatível & & & \\
\hline & Incompatível $\left(\gamma_{06}\right)$ & 0,186 & 0,100 & $(-0,004 ; 0,379)$ \\
\hline & Modelo $T_{E}(\mathrm{R}$ & caída) & & \\
\hline \multirow[t]{3}{*}{ Ano do transplante } & 1985-1989 & & & \\
\hline & $1990-1994\left(\gamma_{11}\right)$ & $-0,517$ & 0,180 & $(-0,865 ;-0,188)$ \\
\hline & $1995-1998\left(\gamma_{12}\right)$ & $-0,348$ & 0,213 & $(-0,735 ; 0,059)$ \\
\hline \multirow{3}{*}{$\begin{array}{l}\text { Idade do paciente } \\
\text { no transplante }\end{array}$} & $\leq 20$ anos & & & \\
\hline & $(20-40]$ anos $\left(\gamma_{13}\right)$ & $-0,126$ & 0,170 & $(-0,474 ; 0,203)$ \\
\hline & $>40 \operatorname{anos}\left(\gamma_{14}\right)$ & 0,263 & 0,219 & $(-0,163 ; 0,697)$ \\
\hline \multirow[t]{2}{*}{ Profilaxia } & Não & & & \\
\hline & $\operatorname{Sim}\left(\gamma_{15}\right)$ & 0,024 & 0,162 & $(-0,322 ; 0,324)$ \\
\hline \multirow{2}{*}{$\begin{array}{l}\text { Compatibilidade sexo } \\
\text { doador-receptor }\end{array}$} & Não incompatível & & & \\
\hline & Incompatível $\left(\gamma_{16}\right)$ & 0,165 & 0,157 & $(-0,117 ; 0,456)$ \\
\hline \multicolumn{5}{|c|}{ Modelo $T_{G}$ (Óbito após recaída) } \\
\hline \multirow[t]{3}{*}{ Ano do transplante } & $1985-1989$ & & & \\
\hline & $1990-1994\left(\gamma_{11}\right)$ & $-0,482$ & 0,140 & $(-0,748 ;-0,204)$ \\
\hline & $1995-1998\left(\gamma_{12}\right)$ & $-0,423$ & 0,164 & $(-0,730 ;-0,098)$ \\
\hline \multirow{3}{*}{$\begin{array}{l}\text { Idade do paciente } \\
\text { no transplante }\end{array}$} & $\leq 20$ anos & & & \\
\hline & $(20-40]$ anos $\left(\gamma_{13}\right)$ & 0,193 & 0,146 & $(-0,061 ; 0,492)$ \\
\hline & $>40 \operatorname{anos}\left(\gamma_{14}\right)$ & 0,249 & 0,183 & $(-0,114 ; 0,580)$ \\
\hline \multirow{2}{*}{ Profilaxia } & Não & & & \\
\hline & $\operatorname{Sim}\left(\gamma_{15}\right)$ & 0,028 & 0,130 & $(-0,198 ; 0,304)$ \\
\hline \multirow{2}{*}{$\begin{array}{l}\text { Compatibilidade sexo } \\
\text { doador-receptor }\end{array}$} & Não incompatível & & & \\
\hline & Incompatível $\left(\gamma_{16}\right)$ & 0,106 & 0,130 & $(-0,131 ; 0,370)$ \\
\hline \multicolumn{5}{|c|}{ Modelo Logito } \\
\hline Intercepto & $\left(\alpha_{0}\right)$ & $-1,584$ & 0,163 & $(-1,902 ;-1,264)$ \\
\hline \multirow[t]{3}{*}{ Ano do transplante } & $1985-1989$ & & & \\
\hline & $1990-1994\left(\alpha_{1}\right)$ & 0,313 & 0,178 & $(-0,006 ; 0,663)$ \\
\hline & $1995-1998\left(\alpha_{2}\right)$ & 0,189 & 0,199 & $(-0,221 ; 0,510)$ \\
\hline \multirow{3}{*}{$\begin{array}{l}\text { Idade do paciente } \\
\text { no transplante }\end{array}$} & $\leq 20$ anos & & & \\
\hline & $\overline{(20-40]}$ anos $\left(\alpha_{3}\right)$ & $-0,100$ & 0,145 & $(-0,365 ; 0,179)$ \\
\hline & $>40 \operatorname{anos}\left(\alpha_{4}\right)$ & $-0,300$ & 0,197 & $(-0,720 ; 0,039)$ \\
\hline \multirow[t]{2}{*}{ Profilaxia } & Não & & & \\
\hline & $\operatorname{Sim}\left(\alpha_{5}\right)$ & 0,252 & 0,152 & $(-0,018 ; 0,574)$ \\
\hline \multirow{2}{*}{$\begin{array}{l}\text { Compatibilidade sexo } \\
\text { doador-receptor }\end{array}$} & Não incompatível & & & \\
\hline & Incompatível $\left(\alpha_{6}\right)$ & $-0,018$ & 0,147 & $(-0,291 ; 0,262)$ \\
\hline Parâmetro Cópula & $\theta$ & 0,986 & 0,259 & $(0,572 ; 1,494)$ \\
\hline
\end{tabular}


direita de zero.

Possíveis interpretações clínicas destes resultados podem ser dadas em termos do risco, tomando-se o exponencial dos parâmetros estimados e comparando com 1 que corresponde ao risco do respectivo grupo de referência. Assim, por exemplo, no modelo $T_{D}$ para o ano de transplante tem-se, para 1990-1994, $\exp \left\{\hat{\gamma_{01}}\right\}=\exp \{-0,509\}=0,601$ e para 1995-1998, $\exp \left\{\hat{\gamma}_{02}\right\}=\exp \{-0,537\}=0,584$ indicando uma diminuição do risco para óbito, em relação aos pacientes transplantados no periodo entre 1985 e 1989. Interpreções similares obtém-se para esta covariável nos modelos $T_{E}$ e $T_{G}$. Quanto à idade, no modelo $T_{D}$, há um incremento do risco para óbito nos indivíduos mayores de 20 anos $(\exp \{0,614\}=1,848$ para indivíduos entre entre 20 e 40 anos e $\exp \{0,947\}=2,577$ para maiores de 40 anos )

A média a posteriori e o HPD de $95 \%$ para o parâmetro da cópula foram 0,986 e $(0,572 ; 1,494)$, respectivamente, o que indica uma dependência moderada entre $T_{E}$ e $T_{G}$. Dado que na cópula de Clayton $\tau=\frac{\theta}{\theta+2}$, uma possível estimativa plug-in pontual de $\tau$ seria 0,33 e intervalar $(0,276 ; 0,428)$.

\subsection{Insuficiência renal crônica (IRC)}

O conjunto de dados consiste dos tempos de acompanhamento de 1252 pacientes com IRC. O acompanhamento foi realizado pelo Fresenius Medical Care de Colombia e Laboratório Médico Echavarria durante um período de 690 dias (período comprendido entre 30 de maio de 2009 e 20 de abril de 2011). Uma das características destes pacientes é que eles se encontram nos estágios mais avançados da doença e, portanto, requerem tratamento dialítico. O tipo de diálise aplicado em cada paciente depende da indicação médica, sendo a hemodiálise o mais comum. Além do tratamento, foram consideradas outras variáveis, registradas no início do seguimento dos pacientes. Uma descrição delas é apresentada Tabela 5.4 e, como pode-se observar, 75\% da amostra está composta por pacientes em tratamento de hemodiálise.

A IRC é uma doença que afeta pessoas de ambos os sexos em todas as idades. Conforme a Tabela 5.4, as maiores proporções são pacientes do sexo masculino, representando $61,3 \%$ da amostra, e pessoas com idades superiores a 50 anos de idade, que correspondem a $64,5 \%$ da amostra.

Ao lidar com a estrutura de riscos semicompetitivos, no estudo de IRC são considerados os eventos de interesse, intermediário e final, como uma piora ou progressão no quadro clínico e o óbito, respectivamente. Segundo a opinião das pesquisadoras do Laboratório Médico Echavarria, fornecedoras dos dados e conforme Steddon e Sharples (2015), existem vários critérios para definir esta piora, geralmente através de exames que avaliam as concentrações de fósforo inorgânico, cálcio, potássio ou nitrogênio uréico (BUN) no sangue. Níveis fora dos estabelecidos como referência indicam a progressão. Especificamente, para pacientes com IRC que iniciam tratamento dialítico, estes níveis são: cálcio, de 8,7 a 10,4 mg/dl; fósforo de 3,4 a 5,6 mg/dl; nitrogênio uréico, de 9 a 23 
Tabela 5.4: Descrição das covariáveis e eventos de interesse do estudo de IRC.

\begin{tabular}{llcc}
\hline \hline & Categorias & $n$ & $\%$ \\
\hline Covariáveis & & & \\
\hline Tratamento & 0 Diálises peritoneal & 309 & 24,68 \\
& 1 Hemodiálises & 943 & 75,32 \\
Sexo & & & \\
& 0 Feminino & 484 & 38,66 \\
& 1 Masculino & 768 & 61,34 \\
Idade & 1 $\leq 30$ anos & 126 & 10,06 \\
& 2 De 31 a 50 anos & 318 & 25,40 \\
& 3 De 51 a 65 anos & 426 & 34,03 \\
& $4 \geq 66$ anos & 382 & 30,51 \\
& & & \\
Cidade & 1 Manizales & 280 & 22,36 \\
& 2 Monteria & 369 & 29,47 \\
& 3 Rionegro & 131 & 10,46 \\
& 4 Sincelejo & 334 & 26,68 \\
& 5 Tunja & 138 & 11,02 \\
\hline Eventos de interesse & & & \\
\hline Caso & 1 Óbito & 177 & 14,14 \\
& 2 Óbito após progressão & 62 & 4,95 \\
& 3 Progressão & 303 & 24,20 \\
& 4 Censura & 710 & 56,71 \\
\hline \hline
\end{tabular}

$\mathrm{mg} / \mathrm{dl}$ e potássio, de 4 a $6 \mathrm{mg} / \mathrm{dl}$.

Eles também indicam que existe uma forte associação entre hiperfosfatemia e mortalidade de pacientes com IRC em ambos os tratamentos dialíticos. Neste trabalho, foi adotado que a progressão da IRC ocorre quando o valor do exame de fósforo inorgânico no sangue for superior ao nível de referência de $5,6 \mathrm{mg} / \mathrm{dl}$.

Na Tabela 5.4 também são resumidas as proporções dos pacientes segundo os eventos de interesse. É importante ressaltar a alta proporção de censura, sendo que os casos três e quatro compõem $80 \%$ da amostra. Esta alta porcentagem de censura poderia dar indícios de presença de curados. No entanto, para a IRC é mais razoável supor que todos os pacientes sejam suscetíveis aos eventos de interesse. Neste caso, a alta censura pode estar relacionada ao fato de o período de acompanhamento ser curto, pois além de alguns pacientes entrarem tardiamente no estudo ${ }^{1}$, ou sairem precocemente ${ }^{2}$, esta doença é considerada de lenta evolução.

\footnotetext{
${ }^{1}$ Os pacientes começaram o seguimento em instantes aleatórios no período do estudo dependendo da data em que foi diagnosticado com estágio avançado da doença e que foi encaminhado a tratamento com diálise.

${ }^{2}$ Pode-se perder contato com o paciente devido a um possível acontecimento aleatório, mudança de plano de saúde, mudança de cidade, etc.
} 
Para ter uma ideia da estrutura dos dados, obtêm-se a distribuição dos pacientes com IRC segundo os eventos de interesse, no período de observação, para cada categoria das covariáveis conforme se resume na Tabela 5.5.

Tabela 5.5: Distribuição do número de pacientes n(\%) com IRC pelas covariáveis segundo os eventos de interesse.

\begin{tabular}{llcccc}
\hline \hline Variável & & Óbito & Óbito após progressão & Progressão & Censura \\
\hline \multirow{2}{*}{ Tratamento } & & & & \\
& 0 Diálises peritoneal & $58(32,8)$ & $12(19,4)$ & $61(20,1)$ & $178(25,1)$ \\
Sexo & 1 Hemodiálises & $119(67,2)$ & $50(80,6)$ & $242(79,9)$ & $532(74,9)$ \\
& & & & & \\
& 0 Feminino & $73(41,2)$ & $28(45,2)$ & $119(39,3)$ & $264(37,2)$ \\
& 1 Masculino & $104(58,8)$ & $34(54,8)$ & $184(60,7)$ & $446(62,8)$ \\
& & & & & \\
& 1 De 1 a 30 anos & $10(5,6)$ & $7(11,3)$ & $39(12,9)$ & $70(9,9)$ \\
& 2 De 31 a 50 anos & $25(14,1)$ & $11(17,7)$ & $95(31,4)$ & $187(26,3)$ \\
& 3 De 51 a 65 anos & $60(33,9)$ & $26(41,9)$ & $114(37,6)$ & $226(31,8)$ \\
& 4 De 66 a 97 anos & $82(46,3)$ & $18(29,0)$ & $55(18,2)$ & $227(32,0)$ \\
& & & & \\
& 1 Manizales & $34(19,2)$ & $10(16,1)$ & $92(30,4)$ & $144(20,3)$ \\
& 2 Monteria & $66(37,3)$ & $23(37,1)$ & $68(22,4)$ & $212(29,9)$ \\
& 3 Rionegro & $9(5,1)$ & $4(6,5)$ & $45(14,9)$ & $73(10,3)$ \\
& 4 Sincelejo & $49(27,7)$ & $17(27,4)$ & $57(18,8)$ & $211(29,7)$ \\
& 5 Tunja & $19(10,7)$ & $8(12,9)$ & $41(13,5)$ & $70(9,9)$ \\
& & & & \\
\hline \hline
\end{tabular}

Da Tabela 5.5 pode-se observar que do total de casos com progressão, $62 / 365=$ $16,98 \%$ apresentaram óbito. Esta porcentagem é relativamente baixa indicando intuitivamente que a possível dependência não será muito alta. Isto também pode ser analisado pelas covariáveis. Veja por exemplo que, por grupos etários, a maior proporção é para os mais idosos ( $\geq 66$ anos), dada por $18 / 73=24,66 \%$, enquanto que a menor é para o grupo de 31 a 50 anos, que é igual a $11 / 106=10,34 \%$. Quanto à cidade, Rionegro apresenta a proporção mais baixa, igual a $4 / 49=8,16 \%$. Visualmente pode-se verificar que a dependência entre os eventos de interesse será baixa e positiva, conforme o gráfico de dispersão dos tempos até a ocorrência de tais eventos apresentado na Figura 5.3.

Pode ressaltar-se também algumas observações por covariáveis. O óbito se apresenta em maior proporção para os mais idosos, mas quando há progressão precedida ao óbito, houve uma maior proporção de pacientes entre 51 e 65 anos. Quanto à cidade, o óbito se apresenta em maior proporção para pacientes de Monteria e a progressão em pacientes de Manizales. 


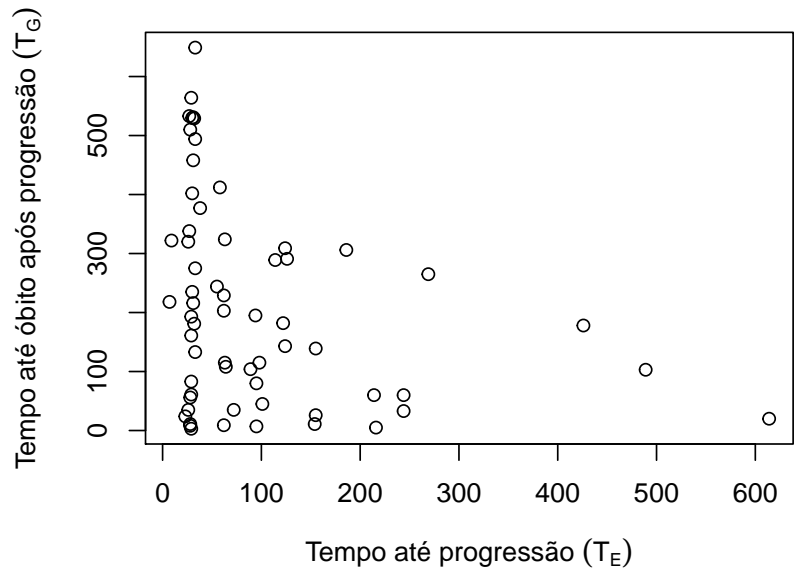

Figura 5.3: Diagrama de dispersão dos tempos até a ocorrência dos eventos de interesse, progressão e óbito, dos dados de IRC. Incluí sómente os indivíduos do caso 2.

Curvas de sobrevivência foram obtidas para os eventos de progressão e óbito seguindo a abordagem de cópulas como indicam Lakhal et al. (2008) e como analisado em Patiño (2012). Especificamente, na Figura 5.4, são apresentadas estas funções usando a cópula de Clayton. Observa-se que as curvas estimadas são diferentes. Para a progressão, a probabilidade de sobrevivência decresce mais rapidamente do que para o óbito ao longo do tempo. Ambos os casos refletem a alta proporção de censura pela forma das curvas ao final do período de acompanhamento, que é uma grande limitação deste estudo.
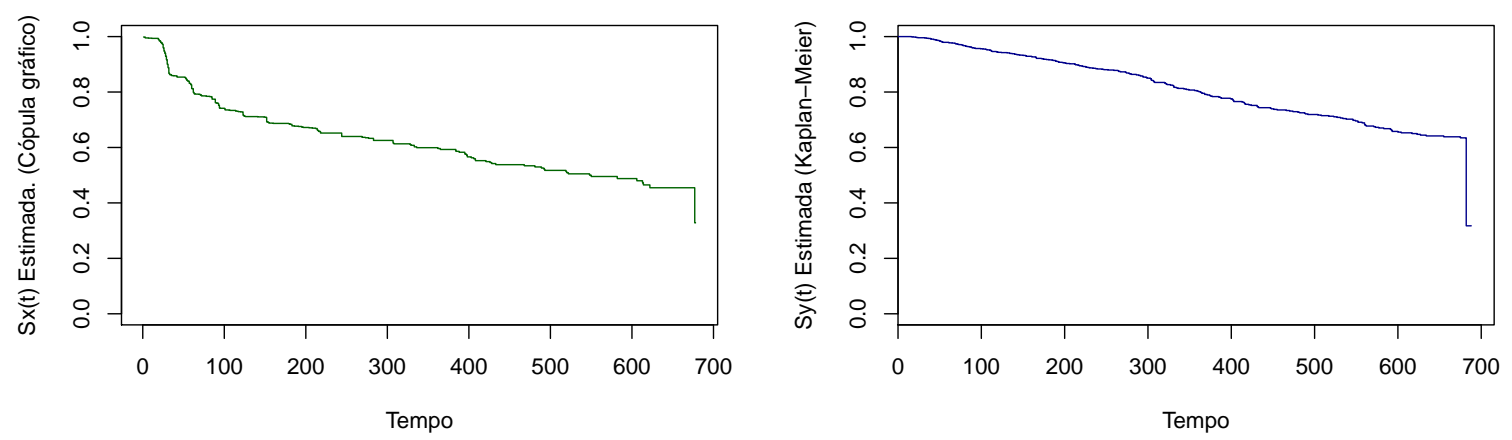

Figura 5.4: Funções de sobrevivência pelo estimador cópula gráfico para o tempo (em dias) até a progressão (esquerda) e Kaplan-Meier para o tempo (em dias) até o óbito (direita).

\subsubsection{Ajuste da modelagem proposta}

Para esta aplicação, foram ajustadas diferentes modelagens fixas e mistas aos dados de IRC variando os valores para as partições das funções de risco basal. Inicialmente considerou-se o ajuste para uma análise paramétrica, ou seja, quando $\left(J_{0}, J_{1}, J_{2}\right)=$ 
$(1,1,1)$ e, para uma análise semiparamétrica foram utilizados diferentes valores para $\left(J_{0}, J_{1}, J_{2}\right)$.

Como comentado na Seção 3.1.2, para todos os modelos ajustados foram adotados como hiperparâmetros para as distribuições a priori os sugeridos na literatura, assim, $\boldsymbol{\alpha} \sim \mathrm{N}_{p_{a}}\left(\mathbf{0}, \boldsymbol{\Sigma}_{a}\right)$ com $\boldsymbol{\Sigma}_{a}=1000 \boldsymbol{I}^{-1} p_{a}$ sendo $\boldsymbol{I}^{-1} p_{a}$ a matriz de identidade inversa de dimensão $p_{a}=10$ para o modelo fixo e $p_{a}=6$ para o modelo misto. Similarmente, $\left(\beta_{0}, \boldsymbol{\gamma}_{0}^{\top}\right)^{\top} \sim \mathrm{N}_{p_{0}}\left(\mathbf{0}, \boldsymbol{\Sigma}_{0}\right),\left(\beta_{1}, \boldsymbol{\gamma}_{1}^{\top}\right) \sim \mathrm{N}_{p_{1}}\left(\mathbf{0}, \boldsymbol{\Sigma}_{1}\right)$ e $\left(\boldsymbol{\beta}_{2}^{\top}, \boldsymbol{\gamma}_{2}^{\top}\right) \sim \mathrm{N}_{p_{2}}\left(\mathbf{0}, \boldsymbol{\Sigma}_{2}\right)$ com $p_{0}=p_{1}=p_{2}=9$ para o modelo fixo e $p_{0}=p_{1}=p_{2}=5$ para o modelo misto. Para $\lambda_{k j}$ são assumidos $\lambda_{k j} \sim \operatorname{Gama}(0,01 ; 0,01)$ para $j=1, \cdots, J_{k}$ e $k=0,1,2$. Para $\theta$ tem-se $\theta \sim \operatorname{GamaInv}(0,01 ; 0,01)$. No caso do modelo misto, para $B$ é assumido $B \sim \operatorname{GamaInv}(3,2)$.

Para todos os modelos ajustados, são usadas 20000 iterações de Gibbs, obtendo amostras de tamanho 750 após um aquecimento de 5000 e espaçamento de 20.

Os resultados das medidas de avaliação de modelos para estes ajustes seguem na Tabela 5.6. Conforme esta tabela, pode-se observar que para o modelo fixo todos os critérios concordam para determinar o modelo $\left(J_{0}, J_{1}, J_{2}\right)=(2,2,2)$ como o melhor ajuste. Já para o modelo misto, o melhor ajuste corresponde à tripla $\left(J_{0}, J_{1}, J_{2}\right)=(1,1,1)$, modelo exponencial.

Tabela 5.6: Valores LPML, BIC DIC para os modelos fixos e mistos segundo a cópula de Clayton ajustados aos dados de IRC.

\begin{tabular}{ccccccccc}
\hline \hline \multicolumn{3}{c}{ Partições } & \multicolumn{3}{c}{ Modelo fixo } & \multicolumn{3}{c}{ Modelo misto } \\
\hline$J_{0}$ & $J_{1}$ & $J_{2}$ & LPML & BIC & DIC & LPML & BIC & DIC \\
\hline & & & & & & & & \\
1 & 1 & 1 & $-1994,211$ & 4204,244 & 3967,719 & $\mathbf{- 1 8 3 0 , 4 4 1}$ & $\mathbf{3 4 5 4 , 2 5 8}$ & $\mathbf{3 9 8 5 , 1 1 4}$ \\
2 & 2 & 2 & $\mathbf{- 1 9 3 8 , 9 7 2}$ & $\mathbf{4 1 2 5 , 7 4 4}$ & $\mathbf{3 8 7 3 , 6 6 4}$ & $-1909,631$ & 3541,668 & 4126,886 \\
3 & 3 & 3 & $-2061,422$ & 4360,655 & 4071,703 & $-2218,365$ & 3788,673 & 4264,081 \\
5 & 5 & 5 & $-2013,732$ & 4307,924 & 3982,387 & $-2108,311$ & 3892,785 & 4413,541 \\
10 & 10 & 10 & $-2465,200$ & 5166,293 & 4660,142 & $-2617,565$ & 4789,746 & 5057,239 \\
2 & 1 & 1 & $-1984,089$ & 4189,906 & 3945,280 & $-1864,477$ & 3512,188 & 4065,928 \\
1 & 2 & 1 & $-1948,454$ & 4134,757 & 3893,606 & $-1858,161$ & 3496,443 & 4047,427 \\
1 & 1 & 2 & $-1990,174$ & 4204,720 & 3958,485 & $-1868,762$ & 3484,176 & 4082,915 \\
1 & 2 & 2 & $-1957,216$ & 4154,570 & 3910,157 & $-1871,909$ & 3536,520 & 4005,753 \\
2 & 2 & 1 & $-1945,106$ & 4127,177 & 3884,937 & $-1860,723$ & 3490,302 & 4006,500 \\
2 & 1 & 2 & $-1985,544$ & 4199,792 & 3953,621 & $-1913,542$ & 3564,155 & 4104,240 \\
1 & 3 & 1 & $-1954,811$ & 4153,613 & 3907,792 & $-2083,916$ & 3611,462 & 4166,226 \\
1 & 3 & 2 & $-1955,996$ & 4161,417 & 3907,930 & $-1958,276$ & 3600,786 & 4190,449 \\
1 & 3 & 5 & $-1958,705$ & 4183,609 & 3913,396 & $-1899,064$ & 3586,022 & 4062,066 \\
& & & & & & & \\
\hline \hline
\end{tabular}

Da Tabela 5.6, pode-se ressaltar que os três critérios, LPML, BIC e DIC, concordam 
na classificação dos modelos fixos ajustados. Para o modelo misto isto ocorre com ao menos dois dos critérios. Observa-se também que ao comparar os ajustes do modelo fixo e misto de uma mesma tripla $\left(J_{0}, J_{1}, J_{2}\right)$, em geral, os critérios fornecem valores menores para o modelo misto. Os respectivos resultados dos ajustes usando a cópula de Clayton, ressaltados na Tabela 5.6, serão apresentados a seguir.

\section{Resultados modelagem fixa}

Nas Tabelas 5.7 e 5.8 são resumidas as estimativas a posteriori (média, desvio padrão (Sd.) e HPD de 95\%) do ajuste do modelo fixo segundo a cópula de Clayton com $\left(J_{0}, J_{1}, J_{2}\right)=(2,2,2)$. Em seguida, no Apêndice D, são apresentadas as Figuras D.5, D.6, D.7 e D.8 que correspondem aos gráficos das funções densidade a posteriori, as sequências dos valores simulados (traços) e as funções de autocorrelação para cada um dos parâmetros das componentes $T_{D}, T_{E}, T_{G}$ e logito, respectivamente. A inferência para o parâmetro da cópula mostra-se na Figura 5.5.

Em todas as situações, é considerado como estimador a média a posteriori.

Considerando que um coeficiente é significativo quando o intervalo de maior densidade a posteriori (HPD) de $95 \%$ não contém o 0, pode ser afirmado das Tabelas 5.7 e 5.8 que o tratamento só parece estar associado à probabilidade da progressão, dado que só se apresentou significativo para a componente logito da modelagem, aqui a chance de progressão é maior para pacientes em tratamento de hemodiálise $(\exp \{1,038\}=2.823)$ que em tratamento de diálise peritoneal $(\exp \{0\}=1)$. Em relação ao sexo, ele tem efeito na sobrevivência dos pacientes que apresentaram óbito, ele foi significativo para as componentes logito e $T_{D}$, em ambos os casos o risco é menor para as pessoas de sexo masculino do que do sexo femenino $(\exp \{-0,543\}=0,581$ no modelo logito e $\exp \{-0,610\}=0,543$ no modelo $\left.T_{D}\right)$.

A idade é outra covariável que aparece como significativa. Em particular, para o grupo etário mais idoso, maiores de 66 anos, no modelo $T_{D}$ quanto no modelo $T_{E}$ sendo que tem um risco maior de óbito e um risco menor de progressão em relação aos mais jovens. No modelo $T_{E}$, em geral, observa-se um risco maior de progressão para os jovens (menores de 30 anos, observa-se que os coeficientes estimados de $\gamma_{12}, \gamma_{13}, \gamma_{14}$, são negativos).

Algumas cidades ou centros de tratamento apresentaram efeitos diferentes nas componentes da modelagem. Monteria apresentou efeito para a progressão, Sincelejo foi significativa para óbito e óbito após progressão (modelo $T_{G}$ ) e Tunja para óbito e a probabilidade de progressão (modelo logito). Observa-se que indivíduos tratados em Manizales tem menor risco de óbito e óbito após progressão que os pacientes tratados nas demais cidades.

Levando em conta que a média a posteriori e o HPD de $95 \%$ para $\theta$ foi 0,143 e $(0,002 ; 0,508)$, respectivamente, pode-se concluir que este parâmetro indica uma dependência positiva entre $T_{E}$ e $T_{G}$, obtendo uma possível estimativa plug-in pontual para 
Tabela 5.7: Ajuste modelo fixo aos dados de IRC com as partições para as funções de risco basal $\left(J_{0}, J_{1}, J_{2}\right)=(2,2,2)$. Componentes: Logito e Modelo $T_{D}$.

\begin{tabular}{|c|c|c|c|c|}
\hline \multicolumn{2}{|c|}{ Covariável / Parâmetro } & Média & Sd. & HPD $95 \%$ \\
\hline \multicolumn{5}{|c|}{ Modelo Logito } \\
\hline Intercepto & $\left(\alpha_{0}\right)$ & $-0,034$ & 0,410 & $(-0,828 ; 0,781)$ \\
\hline \multirow[t]{2}{*}{ Tratamento } & Diálises peritoneal & & & \\
\hline & Hemodiálises $\left(\alpha_{1}\right)$ & 1,038 & 0,337 & $(0,424 ; 1,749)$ \\
\hline \multirow[t]{2}{*}{ Sexo } & Feminino & & & \\
\hline & Masculino $\left(\alpha_{2}\right)$ & $-0,543$ & 0,278 & $(-1,105 ;-0,030)$ \\
\hline \multirow[t]{4}{*}{ Idade } & $\leq 30$ anos & & & \\
\hline & 31 a 50 anos $\left(\alpha_{3}\right)$ & 0,269 & 0,431 & $(-0,497 ; 1,079)$ \\
\hline & 51 a 65 anos $\left(\alpha_{4}\right)$ & $-0,238$ & 0,407 & $(-0,985 ; 0,612)$ \\
\hline & $\geq 66 \operatorname{anos}\left(\alpha_{5}\right)$ & $-0,559$ & 0,425 & $(-1,415 ; 0,232)$ \\
\hline \multirow[t]{5}{*}{ Cidade } & Manizales & & & \\
\hline & Monteria $\left(\alpha_{6}\right)$ & $-0,148$ & 0,394 & $(-0,926 ; 0,564)$ \\
\hline & Rionegro $\left(\alpha_{7}\right)$ & 0,679 & 0,473 & $(-0,359 ; 1,576)$ \\
\hline & Sincelejo $\left(\alpha_{8}\right)$ & $-0,174$ & 0,336 & $(-0,859 ; 0,450)$ \\
\hline & Tunja $\left(\alpha_{9}\right)$ & 0,684 & 0,431 & $(0,123 ; 1,574)$ \\
\hline \multicolumn{5}{|c|}{ Modelo $T_{D}$ (Óbito) } \\
\hline \multirow[t]{2}{*}{ Tratamento } & Diálises peritoneal & & & \\
\hline & Hemodiálises $\left(\beta_{0}\right)$ & 0,120 & 0,271 & $(-0,390 ; 0,630)$ \\
\hline \multirow[t]{2}{*}{ Sexo } & Feminino & & & \\
\hline & Masculino $\left(\gamma_{01}\right)$ & $-0,610$ & 0,230 & $(-1,069 ;-0,153)$ \\
\hline \multirow{3}{*}{ Idade } & $\overline{31}$ a 50 anos $\left(\gamma_{02}\right)$ & 0,018 & 0,516 & $(-0,988 ; 1,029)$ \\
\hline & 51 a $65 \operatorname{anos}\left(\gamma_{03}\right)$ & 0,394 & 0,431 & $(-0,495 ; 1,165)$ \\
\hline & $\geq 66 \operatorname{anos}\left(\gamma_{04}\right)$ & 0,950 & 0,442 & $(0,092 ; 1,775)$ \\
\hline \multirow[t]{5}{*}{ Cidade } & Manizales & & & \\
\hline & Monteria $\left(\gamma_{05}\right)$ & 0,366 & 0,340 & $(-0,255 ; 1,038)$ \\
\hline & Rionegro $\left(\gamma_{06}\right)$ & 0,362 & 0,450 & $(-0,496 ; 1,267)$ \\
\hline & Sincelejo $\left(\gamma_{07}\right)$ & 0,756 & 0,295 & $(0,173 ; 1,300)$ \\
\hline & Tunja $\left(\gamma_{08}\right)$ & 1,412 & 0,475 & $(0,522 ; 2,322)$ \\
\hline
\end{tabular}


Tabela 5.8: Ajuste modelo fixo aos dados de IRC com as partições para as funções de risco basal $\left(J_{0}, J_{1}, J_{2}\right)=(2,2,2)$. Componentes: Modelos $T_{E}$ e $T_{G}$.

\begin{tabular}{|c|c|c|c|c|}
\hline \multicolumn{2}{|c|}{ Covariável / Parâmetro } & Média & $\overline{\mathrm{Sd}}$ & HPD 95\% \\
\hline \multicolumn{5}{|c|}{ Modelo $T_{E}$ (Progressão) } \\
\hline Tratamento & $\begin{array}{l}\text { Diálises peritoneal } \\
\text { Hemodiálises }\left(\beta_{1}\right)\end{array}$ & $-0,474$ & 0,240 & $(-0,921 ; 0,014)$ \\
\hline \multirow[t]{2}{*}{ Sexo } & Feminino & & & \\
\hline & Masculino $\left(\gamma_{11}\right)$ & 0,372 & 0,203 & $(-0,025 ; 0,791)$ \\
\hline \multirow[t]{4}{*}{ Idade } & $\leq 30$ anos & & & \\
\hline & 31 a $50 \operatorname{anos}\left(\gamma_{12}\right)$ & $-0,460$ & 0,268 & $(-0,978 ; 0,064)$ \\
\hline & 51 a 65 anos $\left(\gamma_{13}\right)$ & $-0,250$ & 0,279 & $(-0,755 ; 0,306)$ \\
\hline & $\geq 66 \operatorname{anos}\left(\gamma_{14}\right)$ & $-0,828$ & 0,304 & $(-1,426 ;-0,232)$ \\
\hline \multirow[t]{5}{*}{ Cidade } & Manizales & & & \\
\hline & Monteria $\left(\gamma_{15}\right)$ & $-0,676$ & 0,289 & $(-1,264 ;-0,103)$ \\
\hline & Rionegro $\left(\gamma_{16}\right)$ & 0,045 & 0,269 & $(-0,487 ; 0,534)$ \\
\hline & Sincelejo $\left(\gamma_{17}\right)$ & $-0,408$ & 0,248 & $(-0,897 ; 0,037)$ \\
\hline & Tunja $\left(\gamma_{18}\right)$ & $-0,361$ & 0,266 & $(-0,851 ; 0,190)$ \\
\hline \multicolumn{5}{|c|}{ Modelo $T_{G}$ (Óbito após progressão) } \\
\hline \multirow[t]{2}{*}{ Tratamento } & Diálises peritoneal & & & \\
\hline & Hemodiálises $\left(\beta_{2}\right)$ & 0,050 & 0,341 & $(-0,577 ; 0,730)$ \\
\hline \multirow[t]{2}{*}{ Sexo } & Feminino & & & \\
\hline & Masculino $\left(\gamma_{21}\right)$ & $-0,198$ & 0,265 & $(-0,703 ; 0,299)$ \\
\hline \multirow[t]{4}{*}{ Idade } & $\leq 30$ anos & & & \\
\hline & 31 a $50 \operatorname{anos}\left(\gamma_{22}\right)$ & $-0,197$ & 0,526 & $(-1,174 ; 0,842)$ \\
\hline & 51 a 65 anos $\left(\gamma_{23}\right)$ & 0,365 & 0,487 & $(-0,547 ; 1,297)$ \\
\hline & $\geq 66 \operatorname{anos}\left(\gamma_{24}\right)$ & 0,825 & 0,510 & $(-0,180 ; 1,780)$ \\
\hline \multirow[t]{5}{*}{ Cidade } & Manizales & & & \\
\hline & Monteria $\left(\gamma_{25}\right)$ & 0,810 & 0,387 & $(-0,027 ; 1,517)$ \\
\hline & Rionegro $\left(\gamma_{26}\right)$ & 0,724 & 0,632 & $(-0,461 ; 1,897)$ \\
\hline & Sincelejo $\left(\gamma_{27}\right)$ & 1,569 & 0,414 & $(0,791 ; 2,338)$ \\
\hline & Tunja $\left(\gamma_{28}\right)$ & 0,501 & 0,495 & $(-0,503 ; 1,464)$ \\
\hline Parâmetro Cópula & $\theta$ & 0,143 & 0,178 & $(0,002 ; 0,508)$ \\
\hline
\end{tabular}



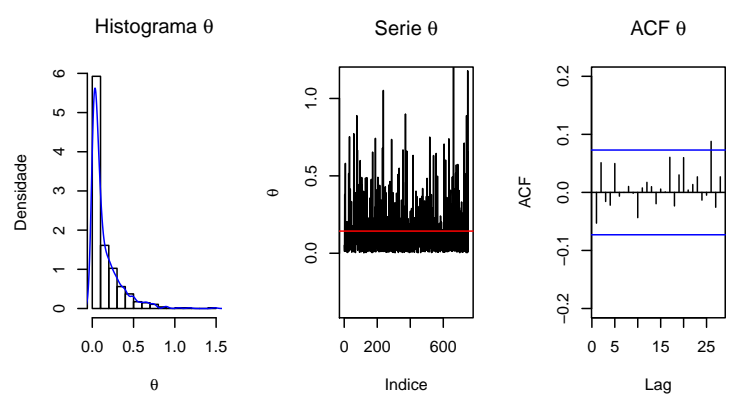

Figura 5.5: Funções densidade a posteriori, traço e ACF para o parâmetro da cópula $\theta$ do ajuste do modelo fixo com $\left(J_{0}, J_{1}, J_{2}\right)=(2,2,2)$ aos dados de IRC.

$\tau$ de $\hat{\tau}=0,143 / 2,143=0,07$ e intervalar de $(0,0009 ; 0,202)$. Embora pequena, não é 0 para poder assumir que os tempos até os eventos de interesse sejam independentes. Neste caso dos pacientes com IRC, o valor pequeno de $\hat{\tau}$ pode ser explicado devido ao fato que, ao tratar com esta doença, caracterizada por ser de lenta evolução, o tempo de acompanhamento é curto e portanto espera-se uma a alta proporção de censura, como de fato foi observado.

Com base nas figuras das funções densidade a posteriori de todos os parâmetros, pode-se incluir as seguintes covariáveis como sendo significativas (considerando que os intervalos HPD contém o 0 na borda direita ou esquerda do intervalo): Para o componente da progressão: Tratamento $\left(\beta_{1}\right)$, grupo etário de 31 - 50 anos $\left(\gamma_{12}\right)$ e a cidade Sincelejo $\left(\gamma_{17}\right)$. Para a componente de óbito após progressão (modelo $T_{G}$ ): a cidades Monteria e $\left(\gamma_{25}\right)$ e Rionegro $\left(\gamma_{26}\right)$. Finalmente para o modelo logito, as cidades Rionegro $\left(\alpha_{7}\right)$ e Sincelejo $\left(\alpha_{8}\right)$.

De forma geral, pode-se concluir que as cidades tem efeito significativo na sobrevivência dos pacientes com IRC e, portanto, é indicativo que um ajuste misto agrupando os dados nas cidades (local de tratamento) deve produzir um melhor ajuste.

\section{Resultados modelo misto}

De forma análoga à modelagem fixa, são resumidas as estimativas a posteriori (média, desvio padrão, HPD 95\% e mediana) do ajuste do modelo misto paramétrico, ou seja, com $\left(J_{0}, J_{1}, J_{2}\right)=(1,1,1)$, conforme Tabela 5.9. Também é assumido como estimativa para todos os parâmetros, a média a posteriori. Os gráficos correspondentes a este ajuste também podem ser consultados no Apêndice D.

Para o ajuste da modelagem são consideradas as seguintes covariáves: tratamento, sexo e grupos etários. Da Tabela 5.9 pode deduzir-se que estas covariáveis resultaram significativas para o modelo de progressão $T_{E}$ dado que 0 não pertence aos intervalos HPD, exceto para o grupo de 51 a 65 anos. No entanto ao observar a respectiva densidade a posteriori na Figura D.10, parâmetro $\gamma_{13}$, o maior volume de massa fica a esquerda de 0 tornando esta variável significativa. Para a componente do óbito após progressão (modelo $T_{G}$ ), o 0 pertence aos intervalos HPD de todos os parâmetros o que fornece 
Tabela 5.9: Ajuste modelo misto aos dados de IRC com as partições para as funções de risco basal $\left(J_{0}, J_{1}, J_{2}\right)=(1,1,1)$.

\begin{tabular}{|c|c|c|c|c|}
\hline \multicolumn{2}{|c|}{ Covariável / Parâmetro } & Média & Desvio P. & HPD $95 \%$ \\
\hline \multicolumn{5}{|c|}{ Modelo $T_{D}$ (Óbito) } \\
\hline Tratamento & $\begin{array}{l}\text { Diálises peritoneal } \\
\text { Hemodiálises }\left(\beta_{0}\right)\end{array}$ & $-0,133$ & 0,176 & $(-0,473 ; 0,220)$ \\
\hline \multirow[t]{2}{*}{ Sexo } & Feminino & & & \\
\hline & $\operatorname{Masculino}\left(\gamma_{01}\right)$ & $-0,331$ & 0,191 & $(-0,673 ; 0,051)$ \\
\hline \multirow[t]{4}{*}{ Idade } & $<30$ anos & & & \\
\hline & 31 a 50 anos $\left(\gamma_{02}\right)$ & $-0,073$ & 0,400 & $(-0,832 ; 0,722)$ \\
\hline & 51 a 65 anos $\left(\gamma_{03}\right)$ & 0,465 & 0,367 & $(-0,269 ; 1,154)$ \\
\hline & $\geq 66 \operatorname{anos}\left(\gamma_{04}\right)$ & 0,835 & 0,363 & $(0,175 ; 1,568)$ \\
\hline \multicolumn{5}{|c|}{ Modelo $T_{E}$ (Progressão) } \\
\hline Tratamento & $\begin{array}{l}\text { Diálises peritoneal } \\
\text { Hemodiálises }\left(\beta_{1}\right)\end{array}$ & $-0,551$ & 0,206 & $(-0,932 ;-0,138)$ \\
\hline \multirow[t]{2}{*}{ Sexo } & Feminino & & & \\
\hline & $\operatorname{Masculino}\left(\gamma_{11}\right)$ & 0,480 & 0,209 & $(0,041 ; 0,829)$ \\
\hline \multirow[t]{4}{*}{ Idade } & $<30$ anos & & & \\
\hline & 31 a 50 anos $\left(\gamma_{12}\right)$ & $-0,529$ & 0,276 & $(-1,067 ;-0,018)$ \\
\hline & 51 a 65 anos $\left(\gamma_{13}\right)$ & $-0,384$ & 0,265 & $(-0,863 ; 0,168)$ \\
\hline & $\geq 66$ anos $\left(\gamma_{14}\right)$ & $-0,618$ & 0,302 & $(-1,239 ;-0,054)$ \\
\hline \multicolumn{5}{|c|}{ Modelo $T_{G}$ (Óbito após Progressão) } \\
\hline Tratamento & $\begin{array}{l}\text { Diálises peritoneal } \\
\text { Hemodiálises }\left(\beta_{2}\right)\end{array}$ & $-0,143$ & 0,333 & $(-0,877 ; 0,418)$ \\
\hline \multirow[t]{2}{*}{ Sexo } & Feminino & & & \\
\hline & Masculino $\left(\gamma_{21}\right)$ & $-0,189$ & 0,276 & $(-0,696 ; 0,333)$ \\
\hline \multirow[t]{4}{*}{ Idade } & $<30$ anos & & & \\
\hline & 31 a 50 anos $\left(\gamma_{22}\right)$ & $-0,310$ & 0,506 & $(-1,251 ; 0,700)$ \\
\hline & 51 a 65 anos $\left(\gamma_{23}\right)$ & 0,381 & 0,449 & $(-0,416 ; 1,312)$ \\
\hline & $\geq 66 \operatorname{anos}\left(\gamma_{24}\right)$ & 0,739 & 0,468 & $(-0,209 ; 1,595)$ \\
\hline \multicolumn{5}{|c|}{ Modelo Logito } \\
\hline \multirow{3}{*}{ Tratamento } & $\alpha_{0}$ (Intercepto) & $-0,333$ & 0,301 & $(-0,868 ; 0,237)$ \\
\hline & Diálises peritoneal & & & \\
\hline & Hemodiálises $\left(\alpha_{1}\right)$ & 0,726 & 0,213 & $(0,339 ; 1,187)$ \\
\hline \multirow[t]{2}{*}{ Sexo } & Feminino & & & \\
\hline & Masculino $\left(\alpha_{2}\right)$ & $-0,376$ & 0,231 & $(-0,880 ; 0,045)$ \\
\hline \multirow[t]{4}{*}{ Idade } & $<30$ anos & & & \\
\hline & 31 a 50 anos $\left(\alpha_{3}\right)$ & 0,004 & 0,325 & $(-0,561 ; 0,689)$ \\
\hline & 51 a 65 anos $\left(\alpha_{4}\right)$ & $-0,202$ & 0,292 & $(-0,756 ; 0,405)$ \\
\hline & 66 a $97 \operatorname{anos}\left(\alpha_{5}\right)$ & $-0,815$ & 0,311 & $(-1,436 ;-0,188)$ \\
\hline Parâmetro Cópula & $\theta$ & 0,107 & 0,124 & $(0,002 ; 0,380)$ \\
\hline Var Efeito aleatório & $B$ & 0,522 & 0,279 & $(0,164 ; 1,111)$ \\
\hline
\end{tabular}


indícios que estas covariáveis não tenham um efeito significativo para esta componente.

No ajuste misto, a covariável sexo tornou-se significativa para a progressão (modelo $T_{E}$ ), além de ter um feito para o óbito (modelo $T_{D}$ ) e a probabilidade de progressão dos pacientes (modelo logito) conforme pode ser visualizado nos gráficos das funções densidade a posteriori, Figura D.9 (parâmetro $\gamma_{01}$ ) e Figura D.12 (parâmetro $\alpha_{2}$ ), respectivamente.

Comparando os resultados obtidos com a modelagem fixa, não houve diferenças nos sinais das estimativas das covariáveis que resultaram significativas. O tratamento, por exemplo, na componente $T_{E}$ em que menores riscos de progressão são obtidos por pessoas tratadas com hemodiálise comparadas com as tratadas com diálise peritoneal. No modelo logito, pessoas de sexo masculino tem menor risco de apresentar progressão do que pessoas de sexo feminino e em relação a idade, os pacientes mais idosos (maiores de 66 anos) tem maior risco de óbito, em tanto que para a progressão seu risco é menor do que os pacientes mais jovens.

Para esta modelagem, o parâmetro $\theta$ associado à dependência entre $T_{E}$ e $T_{G}$ também resultou significativo, obtendo como possíveis estimativas plug-in pontual e intervalar para $\tau$ iguais a $\hat{\tau}=0,107 / 2,107=0,05$ e $(0,0009 ; 0,1597)$. Observe que em relação à modelagem fixa, esta estimativa foi um pouco menor.

Em relação às densidades a posteriori, traços e funções de autocorrelação, tem-se na Figura D.9 os gráficos correspondentes à componente do óbito $T_{D}$. Ao comparar os respectivos parâmetros no ajuste fixo, neste caso misto observam-se cadeias mais estacionárias pois todos os parâmetros desta componente apresentaram desvios menores. As ACFs também apresentaram um melhor comportamento dado que não observamos muitos valores fora das bandas de confiança.

Na Figura D.11 tem-se as densidades a posteriori, traços e ACFs para o modelo $T_{G}$. Esta componente tampouco apresentou diferenças em relação aos parâmetros ajustados no modelo fixo exceto a mudança de sinal na média a posteriori do tratamento.
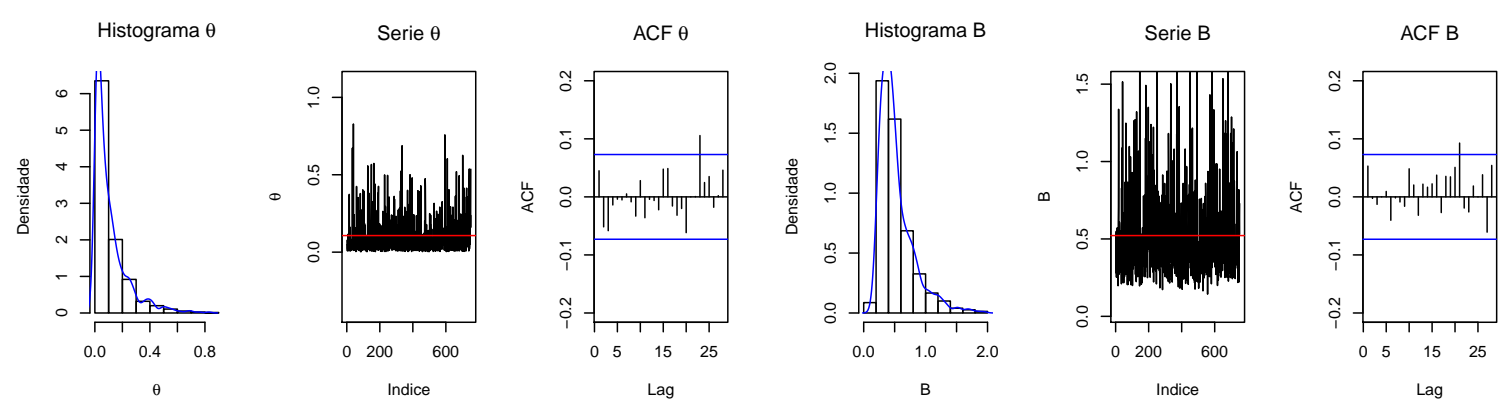

Figura 5.6: Função densidade a posteriori, traço e ACF para o parâmetro da cópula $\theta$ (esquerda) e a variância do efeito aleatório $B$ (direita) do ajuste do modelo misto com $\left(J_{0}, J_{1}, J_{2}\right)=(1,1,1)$ aos dados de IRC. 
No caso do modelo logito os gráficos do ajuste seguem na Figura D.12. Visualmente pode-se observar que as cadeias do modelo misto tiveram um melhor desempenho que o modelo fixo. Como também acontece para o parâmetro da cópula conforme a Figura 5.6.

Quanto à variância do efeito aleatório, cuja estimativa foi 0,522 , pode-se dizer que ela é pequena e possui densidade a posteriori assimétrica (Figura 5.6). Com isso, podese indicar o modelo misto como mais apropriado para os dados do estudo dos pacientes com IRC. 
$\Gamma_{\text {Capítulo }}$

\section{Conclusões}

\subsection{Considerações finais}

Atualmente, em estudos da área médica é muito comum encontrar situações que envolvem eventos não terminais (ou intermediários) e terminais. Dependendo da estrutura do problema e do interesse dos pesquisadores, muitos desses estudos se enquadram na estrutura dos riscos semicompetitivos. Nesta tese propõe-se uma nova modelagem para dados com a estrutura dos riscos semicompetitivos sob a perspectiva bayesiana e considerando cópulas da família Arquimediana e efeito aleatório para incluir dependências entre pacientes tratados em uma mesma clínica ou centro.

A modelagem segue a estrutura do modelo de Zhang et al. (2014) e é motivada pelo estudo de pacientes com insuficiência renal crônica (IRC). Uma análise inicial dos pacientes com IRC, realizada em Patiño (2012), indicou uma possível extensão a um modelo misto com o intuito de agrupar os indivíduos pelo centro de tratamento localizados em diferentes cidades. A inferência é feita sob a abordagem bayesiana e, considerando a cópula de Clayton, é provado o teorema com as condições que garantem que a distribuição a posteriori é própria. A partir da distribuição a posteriori completa, obtida com as variáveis latentes que lidam com a censura e efeito aleatório, são apresentadas as distribuições a posteriori condicionais completas de cada parâmetro. Cada distribuição condicional completa é amostrada pelo algoritmo de fatias para finalmente obter amostras da distribuição a posteriori total segundo o algoritmo de Gibbs.

Toda a implementação computacional foi desenvolvida na linguagem R. Através de um estudo de simulação segundo a cópula de Clayton, verificou-se um desempenho coerente da modelagem levando em conta diferentes situações como proporções de censura, tamanho de amostra e grau de dependência.

Duas aplicações são apresentadas no Capítulo 5. Primeiro, a modelagem fixa (sem efeito aleatório) é aplicada a um conjunto de dados de pacientes com leucemia que receberam transplante de medula óssea. Para tais dados, o modelo misto não se aplica pois não foi medida una variável de agrupamento. Posteriormente, as modelagens fixa e mista são aplicadas aos dados de IRC, os mesmos que fizeram parte da motivação desta 
tese. Para estas aplicações, o melhor ajuste foi escolhido conforme as medidas de comparação de modelos implementadas que, em geral, coincidiam na ordem de classificação dos ajustes. Cabe ressaltar que pela forma dos dados das aplicações aqui analisadas, nenhuma possuia a característica de mudança de tratamento e, portanto, não foi possível avaliar este efeito na modelagem aplicada. Em ambas aplicações encontrou-se dependência fraca positiva entre os tempos até os eventos intermediáios e finais.

De forma geral, pode-se indicar que a inferência bayesiana e a forma de amostragem usada tiveram um bom comportamento. No máximo foram necessárias 20000 iterações do algoritmo de Gibbs para atinguir o regime estacionário das cadeias de Markov nas aplicações. O tempo de execução do programa para as aplicações varia conforme o número de partições $\left(J_{0}, J_{1}, J_{2}\right)$ escolhidas para a estimação das funções de risco basal.

\subsection{Sugestões para pesquisas futuras}

Como perspectiva de pesquisas futuras pretende-se:

- Incluir o efeito aleatório na modelagem para a probabilidade de transição ao estado intermediário. No caso foi usado um modelo logito. No entanto, outras funções de ligação podem ser interessantes como, por exemplo, o modelo probito.

- Considerar diferentes efeitos aleatórios para a ocorrência do evento intermediário $\left(\boldsymbol{b}_{\boldsymbol{E}}\right)$ e o evento terminal $\left(\boldsymbol{b}_{\boldsymbol{G}}\right)$. Isto implicaria considerar diferentes matrizes de variância e covariância.

- Propor uma análise de sensibilidade para a escolha dos hiperparâmetros da distribuição a priori na estimação bayesiana.

- Desenvolver um pacote em R com a implementação da modelagem proposta.

- Obter os resultados teóricos para outras cópulas da família Arquimediana uniparamétrica Frank e Gumbel. As cópulas Frank e Gumbel são consideradas importantes, dado que com elas é possível avaliar dependência negativa e nos extremos, respectivamente. Aqui a ideia é provar que a distribuição a posteriori construída com estas cópulas é própia.

Além disso, a partir desta tese, surgem novas linhas de investigação para os riscos semicompetitivos:

1. Modelo de riscos semicompetitivos com censura à esquerda.

Uma pregunta que caberia se fazer é se o evento intermediário não observado poderia ser uma censura à esquerda.

2. Extensão da modelagem proposta a outras cópulas.

A modelagem explícita da dependência dos eventos de interesse através de cópulas permite dar maior flexibilidade à modelagem. Neste ponto, pode ser de interesse generalizar a modelagem à família biparamétrica Arquimediana Power Variance 
Function (PVF), que inclui as cópulas de Clayton, Gumbel e Gaussiana Inversa. Detalhes da cópula PVF podem ser consultados em Romeo et al. (2018).

3. Modelagem paramétrica.

Ainda na literatura, não se tem uma modelagem parámetrica para a estrutura dos riscos semicompetitivos. Até agora só tem-se modelos de regressão de Cox ou riscos proporcionais.

4. Abordagem clássica.

Abordar a modelagem mista com cópulas segundo o paradigma clássico e estudar as respectivas propriedades assintóticas dos estimadores, além de desenvolver processos de estimação apropriados.

5. Eventos recorrentes.

Estudar uma modelagem para riscos semicompetitivos mais abrangente que inclua eventos recorrentes intermediários e não um só como apresentado nesta tese. 
$\Gamma_{\text {Apêndice }} \boldsymbol{A}$

\section{Função de verossimilhança: Expressões para sua construção usando a cópula de Clayton}

Como discutido na Seção 3.1.1, a função verossimilhança é construída por quatro componentes segundo os possíveis casos.

Primeiramente, veja que do modelo logito (3.1) tem-se que

$$
\begin{aligned}
\operatorname{logit}(\operatorname{Pr}(E=1 \mid A, \boldsymbol{x})) & =\log \left(\frac{\operatorname{Pr}(E=1 \mid A, \boldsymbol{x})}{1-\operatorname{Pr}(E=1 \mid A, \boldsymbol{x})}\right) \\
& =\alpha_{0}+A \alpha_{1}+\boldsymbol{x}^{\top} \boldsymbol{\alpha}_{\mathbf{2}} .
\end{aligned}
$$

Igualando os dois termos à direita da expressão acima e considerando o preditor linear como $K_{P}=\alpha_{0}+A \alpha_{1}+\boldsymbol{x}^{\top} \boldsymbol{\alpha}_{2}$, tem-se $\operatorname{Pr}(E=1 \mid A, \boldsymbol{x}) /(1-\operatorname{Pr}(E=1 \mid$ $A, \boldsymbol{x}))=\exp \left\{K_{P}\right\}$. Logo, isolando $\operatorname{Pr}(E=1 \mid A, \boldsymbol{x})$ obtem-se $\operatorname{Pr}(E=1 \mid A, \boldsymbol{x})=$ $\exp \left\{K_{P}\right\} /\left(1+\exp \left\{K_{P}\right\}\right.$ e, em consequência, $\operatorname{Pr}(E=0 \mid A, \boldsymbol{x})=1-\operatorname{Pr}(E=1 \mid A, \boldsymbol{x})=$ $1 /\left(1+\exp \left\{K_{P}\right\}\right)$. Estas duas probabilidades são usadas nas diferentes expressões que compõem a função verossímilhança, segundo os quatro casos descritos na Seção 3.1.1. A seguir é detalhada a contribuição de cada caso para a modelagem mista.

Caso 1. Os indivíduos apresentam só o evento terminal:

$$
\begin{aligned}
L_{1 i l}\left(\boldsymbol{\alpha}, \beta_{0}, \boldsymbol{\gamma}_{0}, \boldsymbol{\lambda}_{0}, \sigma_{b}^{2} \mid D_{i l}, b_{l}\right) & =\operatorname{Pr}\left(E_{i l}=0 \mid A_{i l}, \boldsymbol{x}_{i l}\right) f_{D}\left(y_{i l} \mid A_{i l}, \boldsymbol{x}_{i l}, b_{l}\right) \\
& =\frac{1}{1+\mathrm{e}^{K_{P i l}}} h_{0}\left(y_{i l} \mid \boldsymbol{\lambda}_{0}\right) \mathrm{e}^{K_{D i l}+b_{l}} \exp \left\{-H_{0}\left(y_{i l} \mid \boldsymbol{\lambda}_{0}\right) \mathrm{e}^{K_{D i l}+b_{l}}\right\} .
\end{aligned}
$$

Esta componente é o produto da probabilidade de que o $i$-ésimo indivíduo do grupo $l$ não apressente progressão e $f_{D}\left(y_{i l} \mid A_{i l}, \boldsymbol{x}_{i l}, b_{l}\right)$, que corresponde à função densidade marginal $f_{D}\left(t_{D} \mid A, \boldsymbol{x}, E=0, b\right)$, derivada da expressão (3.2), que corresponde à função de taxa de falha para óbito. 
Caso 2. Os indivíduos apresentam os dois eventos de interesse: progressão e óbito

$$
\begin{aligned}
& L_{2 i l}\left(\boldsymbol{\alpha}, \beta_{1}, \boldsymbol{\gamma}_{1}, \boldsymbol{\lambda}_{1}, \boldsymbol{\beta}_{2}, \boldsymbol{\gamma}_{2}, \boldsymbol{\lambda}_{2}, \theta, \sigma_{b}^{2} \mid D_{i l}, b_{l}\right)= \\
& \operatorname{Pr}\left(E_{i l}=1 \mid A_{i l}, \boldsymbol{x}_{i l}\right) f_{E G_{\theta}}\left(y_{E i l}, y_{G i l} \mid A_{i l}, V_{i l}, \boldsymbol{x}_{i l}, \boldsymbol{z}_{i l}, b_{l}\right) .
\end{aligned}
$$

Esta componente está composta pelo produto da probabilidade de um indivíduo $i$ do grupo $l$ apresentar progressão, dado o tratamento $A_{i l}$ e as covariáveis basais $\boldsymbol{x}_{i l}$, definidas acima e a função densidade da cópula de Clayton dada pela expressão (2.4).

Para obter a função densidade da cópula de Clayton dadas as covariáveis, ou seja, $f_{E G_{\theta}}\left(y_{E i l}, y_{G i l} \mid A_{i l}, V_{i l}, \boldsymbol{x}_{i l}, \boldsymbol{z}_{i l}, b_{l}\right)$, substituí-se $f_{E}\left(t_{E}\right), f_{G}\left(t_{G}\right), S_{E}\left(t_{E}\right)$ e $S_{G}\left(t_{G}\right)$ em (2.4) pelas respectivas funções densidade e sobrevivência marginais de progressão e do óbito após progressão derivadas das expressões (3.4) e (3.5), respectivamente, para o indivíduo $i$ do grupo $l$.

Assim, por (2.4) tem-se

$$
\begin{aligned}
& f_{E G_{\theta}}\left(y_{E i l}, y_{G i l} \mid A_{i l}, V_{i l}, \boldsymbol{x}_{i l}, \boldsymbol{z}_{i l}, b_{l}\right)= \\
&(1+\theta) f_{E}\left(y_{E i l} \mid A_{i l}, \boldsymbol{x}_{i l}, E_{i l}=1, b_{l}\right) f_{G}\left(y_{G i l} \mid A_{i l}, V_{i l}, \boldsymbol{z}_{i l}, E_{i l}=1, b_{l}\right) \\
& \times\left(S_{E}\left(y_{E i l} \mid A_{i l}, \boldsymbol{x}_{i l}, E_{i l}=1, b_{l}\right) S_{G}\left(y_{G i l} \mid A_{i l}, V_{i l}, \boldsymbol{z}_{i l}, E_{i l}=1, b_{l}\right)\right)^{-(\theta+1)} \\
& \quad \times\left(\left[S_{E}\left(y_{E i l} \mid A_{i l}, \boldsymbol{x}_{i l}, E_{i l}=1, b_{l}\right)\right]^{-\theta}+\left[S_{G}\left(y_{G i l} \mid A_{i l}, V_{i l}, \boldsymbol{z}_{i l}, E_{i l}=1, b_{l}\right)\right]^{-\theta}-1\right)^{-\left(\frac{1}{\theta}+2\right)}
\end{aligned}
$$

Substituíndo

$$
\begin{aligned}
& S_{E}\left(y_{E i l} \mid A_{i l}, \boldsymbol{x}_{i l}, E_{i l}=1, b_{l}\right)=\exp \left\{-H_{1}\left(y_{E i l} \mid \boldsymbol{\lambda}_{1}\right) \exp \left\{K_{E i l}+b_{l}\right\}\right\} ; \\
& f_{E}\left(y_{E i l} \mid A_{i l}, \boldsymbol{x}_{i l}, E_{i l}=1, b_{l}\right)=h_{1}\left(y_{E i l} \mid \boldsymbol{\lambda}_{1}\right) \exp \left\{K_{E i l}+b_{l}-H_{1}\left(y_{E i l} \mid \boldsymbol{\lambda}_{1}\right) \exp \left\{K_{E i l}+b_{l}\right\}\right\}, \\
& S_{G}\left(y_{G i l} \mid A_{i l}, V_{i l}, \boldsymbol{z}_{i l}, E_{i l}=1, b_{l}\right)=\exp \left\{-H_{2}\left(y_{G i l} \mid \boldsymbol{\lambda}_{2}\right) \exp \left\{K_{G}+b_{l}\right\}\right\} \mathrm{e} \\
& f_{G}\left(y_{G i l} \mid A_{i l}, V_{i l}, \boldsymbol{z}_{i l}, E_{i l}=1, b_{l}\right)=h_{2}\left(y_{G i l} \mid \boldsymbol{\lambda}_{2}\right) \exp \left\{K_{G i l}+b_{l}-H_{2}\left(y_{G i l} \mid \boldsymbol{\lambda}_{2}\right) \exp \left\{K_{G i l}+b_{l}\right\}\right\},
\end{aligned}
$$

obtem-se a expressão (3.16) para o $i$-ésimo indivíduo do grupo $l$ dada por

$$
\begin{aligned}
& f_{E G_{\theta}}\left(y_{E i l}, y_{G i l} \mid A_{i l}, V_{i l}, \boldsymbol{x}_{i l}, \boldsymbol{z}_{i l}, b_{l}\right)= \\
& \frac{(1+\theta) h_{1}\left(y_{E i l} \mid \boldsymbol{\lambda}_{1}\right) \mathrm{e}^{K_{E i l}+b_{l}} h_{2}\left(y_{G i l} \mid \boldsymbol{\lambda}_{2}\right) \mathrm{e}^{K_{G i}+b_{l}} \exp \left\{\theta H_{1}\left(y_{E i l} \mid \boldsymbol{\lambda}_{1}\right) \mathrm{e}^{K_{E i l}+b_{l}}+\theta H_{2}\left(y_{G i l} \mid \boldsymbol{\lambda}_{2}\right) \mathrm{e}^{K_{G i l}+b_{l}}\right\}}{\left[\exp \left\{\theta H_{1}\left(y_{E i l} \mid \boldsymbol{\lambda}_{1}\right) \mathrm{e}^{K_{E i l}+b_{l}}\right\}+\exp \left\{\theta H_{2}\left(y_{G i l} \mid \boldsymbol{\lambda}_{2}\right) \mathrm{e}^{K_{G i l}+b_{l}}\right\}-1\right]^{\frac{1}{\theta}+2}} .
\end{aligned}
$$

Caso 3. Os indivíduos apresentam só o evento intermediário da progressão

$$
\begin{aligned}
& L_{3 i l}\left(\boldsymbol{\alpha}, \beta_{1}, \boldsymbol{\gamma}_{1}, \boldsymbol{\lambda}_{1}, \boldsymbol{\beta}_{2}, \boldsymbol{\gamma}_{2}, \boldsymbol{\lambda}_{2}, \theta, \sigma_{b}^{2} \mid D_{i l}, b_{l}\right)= \\
& \quad \operatorname{Pr}\left(E_{i l}=1 \mid A_{i l}, \boldsymbol{x}_{i l}\right) \operatorname{Pr}\left(Y_{E i l}=y_{E i l}, Y_{G i l}>y_{G i l} \mid A_{i l}, V_{i l}, \boldsymbol{x}_{i l}, \boldsymbol{z}_{i l}, b_{l}\right)= \\
& \quad \operatorname{Pr}\left(E_{i l}=1 \mid A_{i l}, \boldsymbol{x}_{i l}\right) \int_{y_{G i l}}^{\infty} f_{E G_{\theta}}\left(y_{E i l}, u \mid A_{i l}, V_{i l}, \boldsymbol{x}_{i l}, \boldsymbol{z}_{i l}, b_{l}\right) d u .
\end{aligned}
$$

Este caso está composto pela probabilidade de progressão, dados o tratamento e as 
covariáveis basais e $\operatorname{Pr}\left(Y_{E i l}=y_{E i l}, Y_{G i l}>y_{G i l} \mid A_{i l}, V_{i l}, \boldsymbol{x}_{i l}, \boldsymbol{z}_{i l}, b_{l}\right)$ indicando que para o indivíduo $i$ do grupo $l$ foi observado o tempo de progressão no instante $y_{E i l}$ e no instante $y_{\text {Gil }}$ não foi observado o evento final, ou seja, sobreviveu ao óbito.

Para obter esta última probabilidade considera-se a expressão geral (3.16), e se calcula $\int_{t_{G}}^{\infty} f_{E G_{\theta}}\left(t_{E}, u\right) d u$ :

$$
\begin{aligned}
\int_{t_{G}}^{\infty} f_{E G_{\theta}}\left(t_{E}, u\right) d u= & (1+\theta) h_{1}\left(t_{E}\right) \exp \left\{K_{E}+b+\theta H_{1}\left(t_{E}\right) \mathrm{e}^{K_{E}+b}\right\} \\
& \int_{t_{G}}^{\infty} h_{2}(u) \mathrm{e}^{K_{G}+b} \exp \left\{\theta H_{2}(u) \mathrm{e}^{K_{G}+b}\right\} \\
& {\left[\exp \left\{\theta H_{1}\left(t_{E}\right) \mathrm{e}^{K_{E}+b}\right\}+\exp \left\{\theta H_{2}(u) \mathrm{e}^{K_{G}+b}\right\}-1\right]^{-\left(\frac{1}{\theta}+2\right)} d u . }
\end{aligned}
$$

Seja $w=\exp \left\{\theta H_{1}\left(t_{E}\right) \mathrm{e}^{K_{E}+b}\right\}+\exp \left\{\theta H_{2}(u) \mathrm{e}^{K_{G}+b}\right\}-1$, de modo que $d w=\exp \left\{\theta H_{2}(u)\right.$ $\left.\mathrm{e}^{K_{G}+b}\right\} \theta h_{2}(u) \mathrm{e}^{K_{G}+b}$. Portanto,

$$
\begin{aligned}
\int_{t_{G}}^{\infty} f_{E G_{\theta}}\left(t_{E}, u\right) d u & =\frac{(1+\theta)}{\theta} h_{1}\left(t_{E}\right) \exp \left\{K_{E}+b+\theta H_{1}\left(t_{E}\right) \mathrm{e}^{K_{E}+b}\right\} \int w^{-\left(\frac{1}{\theta}+2\right)} d w \\
& =\frac{(1+\theta)}{\theta} h_{1}\left(t_{E}\right) \exp \left\{K_{E}+b+\theta H_{1}\left(t_{E}\right) \mathrm{e}^{K_{E}+b}\right\} \frac{1}{-\left(\frac{1}{\theta}+1\right)} \\
& \times\left.\left[\exp \left\{\theta H_{1}\left(t_{E}\right) \mathrm{e}^{K_{E}+b}\right\}+\exp \left\{\theta H_{2}(u) \mathrm{e}^{K_{G}+b}\right\}-1\right]^{-\left(\frac{1}{\theta}+1\right)}\right|_{u=t_{G}} ^{u \rightarrow \infty} \\
& =\frac{h_{1}\left(t_{E}\right) \exp \left\{K_{E}+b+\theta H_{1}\left(t_{E}\right) \mathrm{e}^{K_{E}+b}\right\}}{0+\left[\exp \left\{\theta H_{1}\left(t_{E}\right) \mathrm{e}^{K_{E}+b}\right\}+\exp \left\{\theta H_{2}\left(t_{G}\right) \mathrm{e}^{K_{G}+b}\right\}-1\right]^{\frac{1}{\theta}+1}} .
\end{aligned}
$$

Finalmente, a partir desta expressão temos para o $i$-ésimo indivíduo do grupo $l$,

$$
\begin{aligned}
\int_{y_{G i l}}^{\infty} f_{E G_{\theta}}\left(y_{E i l}, u \mid\right. & \left.A_{i l}, V_{i l}, \boldsymbol{x}_{i l}, \boldsymbol{z}_{i l}, b_{l}\right) d u= \\
& \frac{h_{1}\left(y_{E i l} \mid \boldsymbol{\lambda}_{1}\right) \mathrm{e}^{K_{E i l}+b_{l}} \exp \left\{\theta H_{1}\left(y_{E i l} \mid \boldsymbol{\lambda}_{1}\right) \mathrm{e}^{K_{E i l}+b_{l}}\right\}}{\left[\exp \left\{\theta H_{1}\left(y_{E i l} \mid \boldsymbol{\lambda}_{1}\right) \mathrm{e}^{K_{E i l}+b_{l}}\right\}+\exp \left\{\theta H_{2}\left(y_{G i l} \mid \boldsymbol{\lambda}_{2}\right) \mathrm{e}^{K_{G i}+b_{l}}\right\}-1\right]^{\frac{1}{\theta}+1}} .
\end{aligned}
$$

Caso 4. Os indivíduos neste caso são censurados, nenhum dos dois eventos de interesse é observado

$$
\begin{aligned}
& L_{4 i l}\left(\boldsymbol{\alpha}, \beta_{0}, \boldsymbol{\gamma}_{0}, \boldsymbol{\lambda}_{0}, \beta_{1}, \boldsymbol{\gamma}_{1}, \boldsymbol{\lambda}_{1}, \theta, \sigma_{b}^{2} \mid D_{i l}, b_{l}\right)= \\
& \quad \operatorname{Pr}\left(E_{i l}=1 \mid A_{i l}, \boldsymbol{x}_{i l}\right) S_{E_{\theta}}\left(y_{i l} \mid A_{i l}, \boldsymbol{x}_{i l}\right)+\operatorname{Pr}\left(E_{i l}=0 \mid A_{i l}, \boldsymbol{x}_{i l}\right) S_{D}\left(y_{i l} \mid A_{i l}, \boldsymbol{x}_{i l}\right)= \\
& \quad \operatorname{Pr}\left(E_{i l}=1 \mid A_{i l}, \boldsymbol{x}_{i l}\right) \int_{y_{G i l}}^{\infty} \int_{0}^{\infty} f_{E G_{\theta}}\left(u, v \mid A_{i l}, \boldsymbol{x}_{i l}\right) d v d u \\
& \quad+\operatorname{Pr}\left(E_{i l}=0 \mid A_{i l}, \boldsymbol{x}_{i l}\right) S_{D}\left(y_{i l} \mid A_{i l}, \boldsymbol{x}_{i l}\right) .
\end{aligned}
$$

Este caso está composto por duas partes. Se o indivíduo $i$ do grupo $l$ apresentar progressão tem-se $\operatorname{Pr}\left(E_{i l}=1 \mid A_{i l}, \boldsymbol{x}_{i l}\right) S_{E_{\theta}}\left(y_{i l} \mid A_{i l}, \boldsymbol{x}_{i l}\right)$, caso contrário, a sua contribuição nesta componente será $\operatorname{Pr}\left(E_{i l}=0 \mid A_{i l}, \boldsymbol{x}_{i l}\right) S_{D}\left(y_{i l} \mid A_{i l}, \boldsymbol{x}_{i l}\right)$. 
Observe que as probabilidades de apresentar ou não progressão foram dadas no começo do apêndice e as funções de sobrevivência marginais de óbito e progressão foram obtidas das expressões das funções de taxa de falha (3.2) e (3.4), respectivamente. Particularmente para o indivíduo $i$ do grupo $l$, as expressões para as funções de sobrevivência marginal são dadas por $S_{D}\left(y_{i l} \mid A_{i l}, \boldsymbol{x}_{i l}\right)=\exp \left\{-H_{0}\left(y_{i l} \mid \boldsymbol{\lambda}_{0}\right) \mathrm{e}^{K_{D i l}+b_{l}}\right\}$ e $S_{E_{\theta}}\left(y_{i l} \mid A_{i l}, \boldsymbol{x}_{i l}\right)=\exp \left\{-H_{1}\left(y_{i l} \mid \boldsymbol{\lambda}_{1}\right) \mathrm{e}^{K_{E i l}+b_{l}}\right\}$. Sendo que esta última também pode ser obtida a partir da função de densidade da cópula de Clayton como $S_{E_{\theta}}\left(y_{i l} \mid A_{i l}, \boldsymbol{x}_{i l}\right)=$ $\int_{Y_{G i l}}^{\infty} \int_{0}^{\infty} f_{E G_{\theta}}\left(u, v \mid A_{i l}, \boldsymbol{x}_{i l}\right) d v d u$.

Considerando a expressão geral (3.16) tem-se que

$$
\begin{aligned}
S_{E_{\theta}}(y)= & \int_{y}^{\infty} \int_{0}^{\infty} f_{E G_{\theta}}\left(t_{E}, t_{G}\right) d t_{G} d t_{E} \\
= & \int_{y}^{\infty} \int_{0}^{\infty}(1+\theta) h_{1}\left(t_{E}\right) \mathrm{e}^{K_{E}+b} h_{2}\left(t_{G}\right) \mathrm{e}^{K_{G}+b} \exp \left\{\theta H_{1}\left(t_{E}\right) \mathrm{e}^{K_{E}+b}+\theta H_{2}\left(t_{G}\right) \mathrm{e}^{K_{G}+b}\right\} \\
& \times\left[\exp \left\{\theta H_{1}\left(t_{E}\right) \mathrm{e}^{K_{E}+b}\right\}+\exp \left\{\theta H_{2}\left(t_{G}\right) \mathrm{e}^{K_{G}+b}\right\}-1\right]^{-\left(\frac{1}{\theta}+2\right)} d t_{G} d t_{E} \\
= & \int_{y}^{\infty}(1+\theta) h_{1}\left(t_{E}\right) \mathrm{e}^{K_{E}+b} \exp \left\{\theta H_{1}\left(t_{E}\right) \mathrm{e}^{K_{E}+b}\right\} \int_{0}^{\infty} h_{2}\left(t_{G}\right) \mathrm{e}^{K_{G}+b} \exp \left\{\theta H_{2}\left(t_{G}\right) \mathrm{e}^{K_{G}+b}\right\} \\
& \times\left[\exp \left\{\theta H_{1}\left(t_{E}\right) \mathrm{e}^{K_{E}+b}\right\}+\exp \left\{\theta H_{2}\left(t_{G}\right) \mathrm{e}^{K_{G}+b}\right\}-1\right]^{-\left(\frac{1}{\theta}+2\right)} d t_{G} d t_{E} .
\end{aligned}
$$

Agora, seja $u=\exp \left\{\theta H_{1}\left(t_{E}\right) \mathrm{e}^{K_{E}+b}\right\}+\exp \left\{\theta H_{2}\left(t_{G}\right) \mathrm{e}^{K_{G}+b}\right\}-1$, de modo que $d u=\exp \{\theta$ $\left.H_{2}\left(t_{G}\right) \mathrm{e}^{K_{G}+b}\right\} \theta h_{2}\left(t_{G}\right) \mathrm{e}^{K_{G}+b}$.

Com esta mudança de variável obtém-se

$$
\begin{aligned}
S_{E_{\theta}}(y)= & \int_{y}^{\infty} \int_{0}^{\infty} f_{E G_{\theta}}\left(t_{E}, t_{G}\right) d t_{G} d t_{E} \\
= & \int_{y}^{\infty} \frac{(1+\theta)}{\theta} h_{1}\left(t_{E}\right) \mathrm{e}^{K_{E}+b} \exp \left\{\theta H_{1}\left(t_{E}\right) \mathrm{e}^{K_{E}+b}\right\} \int_{0}^{\infty} u^{-\left(\frac{1}{\theta}+2\right)} d u d t_{E} \\
= & \int_{y}^{\infty} \frac{(1+\theta)}{\theta} h_{1}\left(t_{E}\right) \mathrm{e}^{K_{E}+b} \exp \left\{\theta H_{1}\left(t_{E}\right) \mathrm{e}^{K_{E}+b}\right\}\left(-\frac{\theta}{1+\theta}\right) \\
& \times\left.\left[\exp \left\{\theta H_{1}\left(t_{E}\right) \mathrm{e}^{K_{E}+b}\right\}+\exp \left\{\theta H_{2}\left(t_{G}\right) \mathrm{e}^{K_{G}+b}\right\}-1\right]^{-\left(\frac{1}{\theta}+1\right)}\right|_{t_{G}=0} ^{t_{G} \rightarrow \infty} d t_{E} \\
= & \int_{y}^{\infty} h_{1}\left(t_{E}\right) \mathrm{e}^{K_{E}+b} \exp \left\{\theta H_{1}\left(t_{E}\right) \mathrm{e}^{K_{E}+b}\right\}\left[\exp \left\{\theta H_{1}\left(t_{E}\right) \mathrm{e}^{K_{E}+b}\right\}\right]^{-\left(\frac{1}{\theta}+1\right)} d t_{E}
\end{aligned}
$$

Considere-se agora $w=\exp \left\{\theta H_{1}\left(t_{E}\right) \mathrm{e}^{K_{E}+b}\right\}$, de forma que $d w=\exp \left\{\theta H_{1}\left(t_{E}\right) \mathrm{e}^{K_{G}+b}\right\} \theta h_{1}\left(t_{E}\right)$ $\mathrm{e}^{K_{G}+b}$. Com esta nova mudança de variável, se resolve a integral acima como

$$
\begin{gathered}
S_{E_{\theta}}(y)=\int_{y}^{\infty} h_{1}\left(t_{E}\right) \mathrm{e}^{K_{E}+b} \exp \left\{\theta H_{1}\left(t_{E}\right) \mathrm{e}^{K_{E}+b}\right\}\left[\exp \left\{\theta H_{1}\left(t_{E}\right) \mathrm{e}^{K_{E}+b}\right\}\right]^{-\left(\frac{1}{\theta}+1\right)} d t_{E} \\
=\int w^{-\left(\frac{1}{\theta}+1\right)} d w=\left.\frac{1}{\theta}(-\theta)\left[\exp \left\{\theta H_{1}\left(t_{E}\right) \mathrm{e}^{K_{E}+b}\right\}\right]^{-\frac{1}{\theta}}\right|_{t_{E}=y} ^{t_{E} \rightarrow \infty} .
\end{gathered}
$$


Obtendo

$$
S_{E_{\theta}}(y)=\exp \left\{-H_{1}(y) \mathrm{e}^{K_{E}+b}\right\} .
$$

Juntando tudo obtém-se a contribuição de indivíduos no caso 4 à função verossimilhança

$$
\begin{aligned}
& L_{4 i}\left(\boldsymbol{\alpha}, \beta_{0}, \boldsymbol{\gamma}_{0}, \boldsymbol{\lambda}_{0}, \beta_{1}, \boldsymbol{\gamma}_{1}, \boldsymbol{\lambda}_{1}, \theta \mid D_{i l}, b_{l}\right)= \\
& \quad \frac{\mathrm{e}^{K_{P} i l}}{1+\mathrm{e}^{K_{P} i l}} \exp \left\{-H_{1}\left(y_{i l} \mid \boldsymbol{\lambda}_{1}\right) \mathrm{e}^{K_{E i l}+b_{l}}\right\}+\frac{1}{1+\mathrm{e}^{K_{P} i l}} \exp \left\{-H_{0}\left(y_{i l} \mid \boldsymbol{\lambda}_{0}\right) \mathrm{e}^{K_{D i l}+b_{l}}\right\} .
\end{aligned}
$$


Apêndice $\mathbf{D}$

\section{Prova do teorema 1}

A prova do teorema 1 descrito na Seção 3.2.1 para o modelo misto com a cópula de Clayton é descrita a seguir. Para o modelo fixo, i.e., sem efeito aleatório, não é apresentada aqui dado que é análoga à prova do teorema para o modelo misto com a cópula de Clayton.

\section{$\underline{\text { Prova }}$}

Tem-se que mostrar que, sob as condições do teorema 1, é válida (3.24), ou seja,

$$
\begin{aligned}
& \int_{\Omega^{B}} \int_{\Omega^{\xi}}\left(\prod_{l=1}^{L} \int_{\Omega^{b_{l}}} \prod_{i=1}^{n_{l}}\left[\frac{1}{1+\mathrm{e}^{K_{P i l}}} h_{0}\left(y_{i l} \mid \boldsymbol{\lambda}_{0}\right) \mathrm{e}^{K_{D i l}+b_{l}} \exp \left\{-H_{0}\left(y_{i l} \mid \boldsymbol{\lambda}_{0}\right) \mathrm{e}^{K_{D i l}+b_{l}}\right\}\right]^{\mathbb{I}\left\{\begin{array}{c}
d_{i l}=0 \\
v_{i l}=1
\end{array}\right\}}\right. \\
& \times\left[\frac{\mathrm{e}^{K_{P i l}}}{1+\mathrm{e}^{K_{P i l}}}\left[\exp \left\{\theta H_{1}\left(y_{E i l} \mid \boldsymbol{\lambda}_{1}\right) \mathrm{e}^{K_{E i l}+b_{l}}\right\}+\exp \left\{\theta H_{2}\left(y_{G i l} \mid \boldsymbol{\lambda}_{2}\right) \mathrm{e}^{K_{G i l}+b_{l}}\right\}-1\right]^{-\left(\frac{1}{\theta}+2\right)}(1+\theta)\right. \\
& \left.\times h_{1}\left(y_{E i l} \mid \boldsymbol{\lambda}_{1}\right) \mathrm{e}^{K_{E i l}+b_{l}} h_{2}\left(y_{G i l} \mid \boldsymbol{\lambda}_{2}\right) \mathrm{e}^{K_{G i l}+b_{l}} \exp \left\{\theta H_{1}\left(y_{E i l} \mid \boldsymbol{\lambda}_{1}\right) \mathrm{e}^{K_{E i l}+b_{l}}+\theta H_{2}\left(y_{G i l} \mid \boldsymbol{\lambda}_{2}\right) \mathrm{e}^{K_{G i l}+b_{l}}\right\}\right]^{\mathbb{I}\left\{\begin{array}{c}
d_{i l}=1 \\
v_{i l}=1
\end{array}\right\}} \\
& \times\left[\frac{\mathrm{e}^{K_{P i l}}}{1+\mathrm{e}^{K_{P i l}}} \frac{h_{1}\left(y_{E i l} \mid \boldsymbol{\lambda}_{1}\right) \mathrm{e}^{K_{E i l}+b_{l}} \exp \left\{\theta H_{1}\left(y_{E i l} \mid \boldsymbol{\lambda}_{1}\right) \mathrm{e}^{K_{E i l}+b_{l}}\right\}}{\left[\exp \left\{\theta H_{1}\left(y_{E i l} \mid \boldsymbol{\lambda}_{1}\right) \mathrm{e}^{K_{E i l}+b_{l}}\right\}+\exp \left\{\theta H_{2}\left(y_{G i l} \mid \boldsymbol{\lambda}_{2}\right) \mathrm{e}^{K_{G i l}+b_{l}}\right\}-1\right]^{\frac{1}{\theta}+1}}\right]^{\mathbb{I}\left\{\begin{array}{c}
d_{i l}=1 \\
v_{i l}=0
\end{array}\right\}} \\
& \times\left[\frac{\mathrm{e}^{K_{P} i l}}{1+\mathrm{e}^{K_{P} i l}} \exp \left\{-H_{1}\left(y_{i l} \mid \boldsymbol{\lambda}_{1}\right) \mathrm{e}^{K_{E i l}+b_{l}}\right\}+\frac{1}{1+\mathrm{e}^{K_{P} i l}} \exp \left\{-H_{0}\left(y_{i l} \mid \boldsymbol{\lambda}_{0}\right) \mathrm{e}^{K_{D i l}+b_{l}}\right\}\right]^{\mathbb{I}\left\{\begin{array}{c}
d_{i l}=0 \\
v_{i l}=0
\end{array}\right\}} \\
& \left.\times(2 \pi B)^{-\frac{1}{2}} \exp \left\{-\frac{b_{l}^{2}}{2 B}\right\} d b_{l}\right) \pi(\boldsymbol{\alpha}) \pi\left(\beta_{0}, \gamma_{0}\right) \pi\left(\beta_{1}, \gamma_{1}\right) \pi\left(\boldsymbol{\beta}_{2}, \gamma_{2}\right) \prod_{j=1}^{J_{0}} \frac{1}{\lambda_{0 j}} \prod_{j=1}^{J_{1}} \frac{1}{\lambda_{1 j}} \\
& \times \prod_{j=1}^{J_{2}} \frac{1}{\lambda_{2 j}}\left[\frac{\exp \left\{-\frac{\varsigma_{\theta}}{\theta}\right\}}{\theta^{\kappa_{\theta}+1}}\right]\left[\frac{\exp \left\{-\frac{\varsigma_{B}}{B}\right\}}{B^{\kappa_{B}+1}}\right] d \boldsymbol{\alpha} d \beta_{0} d \boldsymbol{\gamma}_{0} d \beta_{1} d \boldsymbol{\gamma}_{1} d \boldsymbol{\beta}_{2} d \boldsymbol{\gamma}_{2} d \boldsymbol{\lambda}_{\mathbf{0}} d \boldsymbol{\lambda}_{\mathbf{1}} d \boldsymbol{\lambda}_{\mathbf{2}} d \theta d B<\infty .
\end{aligned}
$$

Relembre que o vetor de parâmetros é $\boldsymbol{\xi}=\left(\boldsymbol{\alpha}^{\top}, \beta_{0}, \boldsymbol{\gamma}_{0}^{\top}, \boldsymbol{\lambda}_{0}^{\top}, \beta_{1}, \boldsymbol{\gamma}_{1}^{\top}, \boldsymbol{\lambda}_{1}^{\top}, \boldsymbol{\beta}_{2}^{\top}, \boldsymbol{\gamma}_{2}^{\top}, \boldsymbol{\lambda}_{2}^{\top}, \theta\right)^{\top}$, $B$ é a variância do efeito aleatório e $\boldsymbol{b}=\left(b_{1}, \cdots, b_{l}\right)^{\top}$ o vetor dos L efeitos aleatórios. 
Pelo teorema de Tonelli e considerando que o integrando é não negativo, a ordem de integração pode mudar. Além disso, a componente do modelo logito pode ser isolada nos casos 1, 2 e 3, obtendo-se a expressão

$$
\begin{aligned}
& \int_{\Omega^{B}} \int_{\Omega^{\boldsymbol{b}}} \int_{\Omega^{\boldsymbol{\alpha}}} \prod_{i \in \cup_{m=1}^{3} \mathfrak{N}_{m}, \forall l} \frac{\mathrm{e}^{K_{P i l} E_{i l}}}{1+\mathrm{e}^{K_{P i l}}} \pi(\boldsymbol{\alpha}) d \boldsymbol{\alpha}\left(\int _ { \Omega ^ { \boldsymbol { \xi } - \{ \boldsymbol { \alpha } \} } } \left(\prod _ { l = 1 } ^ { L } \prod _ { i = 1 } ^ { n _ { l } } \left[h_{0}\left(y_{i l} \mid \boldsymbol{\lambda}_{0}\right) \mathrm{e}^{K_{D i l}+b_{l}}\right.\right.\right. \\
& \left.\times \exp \left\{-H_{0}\left(y_{i l} \mid \boldsymbol{\lambda}_{0}\right) \mathrm{e}^{K_{D i l}+b_{l}}\right\}\right]^{\mathbb{I}\left\{\begin{array}{l}
\left\{_{i l l}=0\right. \\
v_{i l}=1
\end{array}\right.}\left[\left[\exp \left\{\theta H_{1}\left(y_{E i l} \mid \boldsymbol{\lambda}_{1}\right) \mathrm{e}^{K_{E i l}+b_{l}}\right\}+\exp \left\{\theta H_{2}\left(y_{G i l} \mid \boldsymbol{\lambda}_{2}\right)\right.\right.\right. \\
& \left.\left.\times \mathrm{e}^{K_{G i l}+b_{l}}\right\}-1\right]^{-\left(\frac{1}{\theta}+2\right)}(1+\theta) h_{1}\left(y_{E i l} \mid \boldsymbol{\lambda}_{1}\right) \mathrm{e}^{K_{E i l}+b_{l}} h_{2}\left(y_{G i l} \mid \boldsymbol{\lambda}_{2}\right) \mathrm{e}^{K_{G i l}+b_{l}} \exp \left\{\theta H_{1}\left(y_{E i l} \mid \boldsymbol{\lambda}_{1}\right)\right. \\
& \left.\left.\times \mathrm{e}^{K_{E i l}+b_{l}}+\theta H_{2}\left(y_{G i l} \mid \boldsymbol{\lambda}_{2}\right) \mathrm{e}^{K_{G i l}+b_{l}}\right\}\right]^{\mathbb{I}\left\{\begin{array}{l}
d_{i l}=1 \\
v_{i l}=1
\end{array}\right\}} \\
& \times\left[\frac{h_{1}\left(y_{E i l} \mid \boldsymbol{\lambda}_{1}\right) \mathrm{e}^{K_{E i l}+b_{l}} \exp \left\{\theta H_{1}\left(y_{E i l} \mid \boldsymbol{\lambda}_{1}\right) \mathrm{e}^{K_{E i l}+b_{l}}\right\}}{\left[\exp \left\{\theta H_{1}\left(y_{E i l} \mid \boldsymbol{\lambda}_{1}\right) \mathrm{e}^{K_{E i l}+b_{l}}\right\}+\exp \left\{\theta H_{2}\left(y_{\text {Gil }} \mid \boldsymbol{\lambda}_{2}\right) \mathrm{e}^{K_{G i l}+b_{l}}\right\}-1\right]^{\frac{1}{\theta}+1}}\right]^{\mathbb{I}\left\{\begin{array}{l}
d_{i l}=1 \\
v_{i l}=0
\end{array}\right\}} \\
& \times\left[\frac{\mathrm{e}^{K_{P} i l}}{1+\mathrm{e}^{K_{P} i l}} \exp \left\{-H_{1}\left(y_{i l} \mid \boldsymbol{\lambda}_{1}\right) \mathrm{e}^{K_{E i l}+b_{l}}\right\}+\frac{1}{1+\mathrm{e}^{K_{P} i l}} \exp \left\{-H_{0}\left(y_{i l} \mid \boldsymbol{\lambda}_{0}\right) \mathrm{e}^{K_{D i l}+b_{l}}\right\}\right]^{\mathbb{I}\left\{\begin{array}{c}
d_{i l}=0 \\
v_{i l}=0
\end{array}\right\}} \\
& \left.\times(2 \pi B)^{-\frac{1}{2}} \exp \left\{-\frac{b_{l}^{2}}{2 B}\right\}\right) \pi\left(\beta_{0}, \boldsymbol{\gamma}_{0}\right) \pi\left(\beta_{1}, \boldsymbol{\gamma}_{1}\right) \pi\left(\boldsymbol{\beta}_{2}, \boldsymbol{\gamma}_{2}\right) \prod_{j=1}^{J_{0}} \frac{1}{\lambda_{0 j}} \prod_{j=1}^{J_{1}} \frac{1}{\lambda_{1 j}} \prod_{j=1}^{J_{2}} \frac{1}{\lambda_{2 j}} \\
& \left.\times\left[\frac{\exp \left\{-\frac{\varsigma_{\theta}}{\theta}\right\}}{\theta^{\kappa_{\theta}+1}}\right]\left[\frac{\exp \left\{-\frac{\varsigma_{B}}{B}\right\}}{B^{\kappa_{B}+1}}\right] d \beta_{0} d \boldsymbol{\gamma}_{0} b \beta_{1} d \boldsymbol{\gamma}_{1} d \boldsymbol{\beta}_{2} d \boldsymbol{\gamma}_{2} d \boldsymbol{\lambda}_{\mathbf{0}} d \boldsymbol{\lambda}_{\mathbf{1}} d \boldsymbol{\lambda}_{\mathbf{2}} d \theta\right) d \boldsymbol{b} d B .
\end{aligned}
$$

Considerando as expressões

$$
\begin{aligned}
& L_{3 i l}\left(\beta_{1}, \boldsymbol{\gamma}_{1}, \boldsymbol{\lambda}_{1}, \boldsymbol{\beta}_{2}, \boldsymbol{\gamma}_{2}, \boldsymbol{\lambda}_{2}, \theta \mid D_{i l}, b_{l}\right)= \\
& \frac{h_{1}\left(y_{E i l} \mid \boldsymbol{\lambda}_{1}\right) \mathrm{e}^{K_{E i l}+b_{l}} \exp \left\{\theta H_{1}\left(y_{E i l} \mid \boldsymbol{\lambda}_{1}\right) \mathrm{e}^{K_{E i l}+b_{l}}\right\}}{\left[\exp \left\{\theta H_{1}\left(y_{E i l} \mid \boldsymbol{\lambda}_{1}\right) \mathrm{e}^{K_{E i l}+b_{l}}\right\}+\exp \left\{\theta H_{2}\left(y_{G i l} \mid \boldsymbol{\lambda}_{2}\right) \mathrm{e}^{K_{G i l}+b_{l}}\right\}-1\right]^{\frac{1}{\theta}+1}} \\
& L_{4 i l}\left(\boldsymbol{\alpha}, \beta_{0}, \boldsymbol{\gamma}_{0}, \boldsymbol{\lambda}_{0}, \beta_{1}, \boldsymbol{\gamma}_{1}, \boldsymbol{\lambda}_{1}, \theta \mid D_{i l}, b_{l}\right)= \\
& \frac{\mathrm{e}^{K_{P} i l}}{1+\mathrm{e}^{K_{P} i l}} \exp \left\{-H_{1}\left(y_{i l} \mid \boldsymbol{\lambda}_{1}\right) \mathrm{e}^{K_{E i l}+b_{l}}\right\}+\frac{1}{1+\mathrm{e}^{K_{P} i l}} \exp \left\{-H_{0}\left(y_{i l} \mid \boldsymbol{\lambda}_{0}\right) \mathrm{e}^{K_{D i l}+b_{l}}\right\},
\end{aligned}
$$

observe que $\theta H_{2}\left(y_{G i l} \mid \boldsymbol{\lambda}_{2}\right) \mathrm{e}^{K_{G i l}+b_{l}} \geq 0$, então $\exp \left\{\theta H_{2}\left(y_{G i l} \mid \boldsymbol{\lambda}_{2}\right) \mathrm{e}^{K_{G i}+b_{l}}\right\} \geq 1$, de modo que

$$
L_{3 i l}\left(\cdot \mid D_{i l}, b_{l}\right) \leq \frac{h_{1}\left(y_{E i l} \mid \boldsymbol{\lambda}_{1}\right) \mathrm{e}^{K_{E i l}+b_{l}} \exp \left\{\theta H_{1}\left(y_{E i l} \mid \boldsymbol{\lambda}_{1}\right) \mathrm{e}^{K_{E i l}+b_{l}}\right\}}{\left[\exp \left\{\theta H_{1}\left(y_{E i l} \mid \boldsymbol{\lambda}_{1}\right) \mathrm{e}^{K_{E i}+b_{l}}\right\}\right]^{\frac{1}{\theta}+1}}=\frac{h_{1}\left(y_{E i l} \mid \boldsymbol{\lambda}_{1}\right) \mathrm{e}^{K_{E i l}+b_{l}}}{\exp \left\{H_{1}\left(y_{E i l} \mid \boldsymbol{\lambda}_{1}\right) \mathrm{e}^{K_{E i}+b_{l}}\right\}} .
$$

Também note que $L_{4 i l}\left(\boldsymbol{\alpha}, \beta_{0}, \boldsymbol{\gamma}_{0}, \boldsymbol{\lambda}_{0}, \beta_{1}, \boldsymbol{\gamma}_{1}, \boldsymbol{\lambda}_{1} \mid D_{i l}, b_{l}\right) \leq 1$. 
Portanto, seja

$$
\begin{aligned}
& A=\prod_{l=1}^{L} \prod_{i=1}^{n_{l}}\left([ h _ { 0 } ( y _ { i l } | \boldsymbol { \lambda } _ { 0 } ) \mathrm { e } ^ { K _ { D i l } + b _ { l } } \operatorname { e x p } \{ - H _ { 0 } ( y _ { i l } | \boldsymbol { \lambda } _ { 0 } ) \mathrm { e } ^ { K _ { D i l } + b _ { l } } \} ] ^ { \mathbb { I } \{ \begin{array} { c } 
{ d _ { i l } = 0 } \\
{ v _ { i l } = 1 }
\end{array} } \left[\left[\exp \left\{\theta H_{1}\left(y_{E i l} \mid \boldsymbol{\lambda}_{1}\right) \mathrm{e}^{K_{E i l}+b_{l}}\right\}\right.\right.\right. \\
& \left.+\exp \left\{\theta H_{2}\left(y_{G i l} \mid \boldsymbol{\lambda}_{2}\right) \mathrm{e}^{K_{G i l}+b_{l}}\right\}-1\right]^{-\left(\frac{1}{\theta}+2\right)}(1+\theta) h_{1}\left(y_{E i l} \mid \boldsymbol{\lambda}_{1}\right) \mathrm{e}^{K_{E i l}+b_{l}} h_{2}\left(y_{G i l} \mid \boldsymbol{\lambda}_{2}\right) \mathrm{e}^{K_{G i l}+b_{l}} \\
& \left.\times \exp \left\{\theta H_{1}\left(y_{E i l} \mid \boldsymbol{\lambda}_{1}\right) \mathrm{e}^{K_{E i l}+b_{l}}+\theta H_{2}\left(y_{G i l} \mid \boldsymbol{\lambda}_{2}\right) \mathrm{e}^{K_{G i l}+b_{l}}\right\}\right]^{\mathbb{I}\left\{\begin{array}{l}
d_{i l}=1 \\
v_{i l}=1
\end{array}\right\}} \\
& \times\left[\frac{h_{1}\left(y_{E i l} \mid \boldsymbol{\lambda}_{1}\right) \mathrm{e}^{K_{E i l}+b_{l}} \exp \left\{\theta H_{1}\left(y_{E i l} \mid \boldsymbol{\lambda}_{1}\right) \mathrm{e}^{K_{E i l}+b_{l}}\right\}}{\left[\exp \left\{\theta H_{1}\left(y_{E i l} \mid \boldsymbol{\lambda}_{1}\right) \mathrm{e}^{K_{E i l}+b_{l}}\right\}+\exp \left\{\theta H_{2}\left(y_{G i l} \mid \boldsymbol{\lambda}_{2}\right) \mathrm{e}^{K_{G i}+b_{l}}\right\}-1\right]^{\frac{1}{\theta}+1}}\right]^{\mathbb{I}\left\{\begin{array}{l}
d_{i l}=1 \\
v_{i l}=0
\end{array}\right.}\left[\frac{\mathrm{e}^{K_{P} i l}}{1+\mathrm{e}^{K_{P} i l}}\right. \\
& \left.\times \exp \left\{-H_{1}\left(y_{i l} \mid \boldsymbol{\lambda}_{1}\right) \mathrm{e}^{K_{E i l}+b_{l}}\right\}+\frac{1}{1+\mathrm{e}^{K_{P} i l}} \exp \left\{-H_{0}\left(y_{i l} \mid \boldsymbol{\lambda}_{0}\right) \mathrm{e}^{K_{D i l}+b_{l}}\right\}\right]^{\mathbb{I}\left\{\begin{array}{l}
d_{i l}=0 \\
v_{i l}=0
\end{array}\right.}(2 \pi B)^{-\frac{1}{2}} \\
& \left.\times \exp \left\{-\frac{b_{l}^{2}}{2 B}\right\}\right)
\end{aligned}
$$

que corresponde à expressão entre parênteses da integral interna da expressão (B.2) e então, observe que

$$
\begin{aligned}
A & \leq \prod_{l=1}^{L} \prod_{i=1}^{n_{l}}\left([ h _ { 0 } ( y _ { i l } | \boldsymbol { \lambda } _ { 0 } ) \mathrm { e } ^ { K _ { D i l } + b _ { l } } \operatorname { e x p } \{ - H _ { 0 } ( y _ { i l } | \boldsymbol { \lambda } _ { 0 } ) \mathrm { e } ^ { K _ { D i l } + b _ { l } } \} ] ^ { \mathbb { I } \{ \{ _ { v _ { i } = 1 } ^ { d _ { i } = 0 } \} } \left[\left[\exp \left\{\theta H_{1}\left(y_{E i l} \mid \boldsymbol{\lambda}_{1}\right) \mathrm{e}^{K_{E i l}+b_{l}}\right\}\right.\right.\right. \\
& \left.+\exp \left\{\theta H_{2}\left(y_{G i l} \mid \boldsymbol{\lambda}_{2}\right) \mathrm{e}^{K_{G i l}+b_{l}}\right\}-1\right]^{-\left(\frac{1}{\theta}+2\right)}(1+\theta) h_{1}\left(y_{E i l} \mid \boldsymbol{\lambda}_{1}\right) \mathrm{e}^{K_{E i l}+b_{l}} h_{2}\left(y_{G i l} \mid \boldsymbol{\lambda}_{2}\right) \mathrm{e}^{K_{G i l}+b_{l}} \\
& \left.\times \exp \left\{\theta H_{1}\left(y_{E i l} \mid \boldsymbol{\lambda}_{1}\right) \mathrm{e}^{K_{E i l}+b_{l}}+\theta H_{2}\left(y_{G i l} \mid \boldsymbol{\lambda}_{2}\right) \mathrm{e}^{K_{G i l}+b_{l}}\right\}\right]^{\mathbb{I}\left\{d_{i l l}^{d_{l i}=1}=1\right.}\left[\frac{h_{1}\left(y_{E i l} \mid \boldsymbol{\lambda}_{1}\right) \mathrm{e}^{K_{E i l}+b_{l}}}{\exp \left\{H_{1}\left(y_{E i l} \mid \boldsymbol{\lambda}_{1}\right) \mathrm{e}^{K_{E i l}+b_{l}}\right\}}\right]^{\mathbb{I}\left\{\begin{array}{l}
d_{i}=1 \\
v_{i}=0
\end{array}\right.} \\
& \left.\times(2 \pi B)^{-\frac{1}{2}} \exp \left\{-\frac{b_{l}^{2}}{2 B}\right\}\right) .
\end{aligned}
$$

A expressão do lado direito desta desigualdade inclui os indivíduos que apresentam pelo menos um dos eventos de interesse, sendo então uma cota superior para A, e assim para provar (B.1), basta verificar que

(i)

$$
\int_{\Omega^{\alpha}} \prod_{i \in \cup_{m=1}^{3} \mathcal{N}_{m}, \forall l} \frac{\mathrm{e}^{K_{P i l} E_{i l}}}{1+\mathrm{e}^{K_{P i l}}} \pi(\boldsymbol{\alpha}) d \boldsymbol{\alpha}=\int_{\Omega^{\alpha}} \mathrm{L}_{a}\left(\boldsymbol{\alpha} \mid D_{o b s}\right) \pi(\boldsymbol{\alpha}) d(\boldsymbol{\alpha})<\infty e
$$

(ii)

$$
\begin{aligned}
& \int_{\Omega^{B}} \int_{\Omega^{b}}\left(\int _ { \Omega ^ { \boldsymbol { \xi } - \{ \boldsymbol { \alpha } \} } } \left(\prod_{l=1}^{L} \prod_{i=1}^{n_{l}}\left[h_{0}\left(y_{i l} \mid \boldsymbol{\lambda}_{0}\right) \mathrm{e}^{K_{D i l}+b_{l}} \exp \left\{-H_{0}\left(y_{i l} \mid \boldsymbol{\lambda}_{0}\right) \mathrm{e}^{K_{D i l}+b_{l}}\right\}\right]^{\mathbb{I}\left\{\begin{array}{l}
d_{i l l}=0 \\
v_{i l}=1
\end{array}\right\}}[\exp \{\theta\right.\right. \\
& \left.\left.\times H_{1}\left(y_{E i l} \mid \boldsymbol{\lambda}_{1}\right) \mathrm{e}^{K_{E i l}+b_{l}}\right\}+\exp \left\{\theta H_{2}\left(y_{G i l} \mid \boldsymbol{\lambda}_{2}\right) \mathrm{e}^{K_{G i l}+b_{l}}\right\}-1\right]^{-\left(\frac{1}{\theta}+2\right)}(1+\theta) h_{1}\left(y_{E i l} \mid \boldsymbol{\lambda}_{1}\right) \mathrm{e}^{K_{E i l}+b_{l}} \\
& \left.\times h_{2}\left(y_{G i l} \mid \boldsymbol{\lambda}_{2}\right) \mathrm{e}^{K_{G i l}+b_{l}} \exp \left\{\theta H_{1}\left(y_{E i l} \mid \boldsymbol{\lambda}_{1}\right) \mathrm{e}^{K_{E i l}+b_{l}}+\theta H_{2}\left(y_{G i l} \mid \boldsymbol{\lambda}_{2}\right) \mathrm{e}^{K_{G i l}+b_{l}}\right\}\right]^{\mathbb{I}\left\{\begin{array}{l}
d_{i l}=1 \\
v_{i l}=1
\end{array}\right\}}
\end{aligned}
$$




$$
\begin{aligned}
& \left.\times\left[\frac{h_{1}\left(y_{E i l} \mid \boldsymbol{\lambda}_{1}\right) \mathrm{e}^{K_{E i l}+b_{l}}}{\exp \left\{H_{1}\left(y_{E i l} \mid \boldsymbol{\lambda}_{1}\right) \mathrm{e}^{K_{E i l}+b_{l}}\right\}}\right]^{\mathbb{I}\left\{\begin{array}{l}
\left\{_{i l}=1\right. \\
v_{i l}=0
\end{array}\right.}(2 \pi B)^{-\frac{1}{2}} \exp \left\{-\frac{b_{l}^{2}}{2 B}\right\}\right) \pi\left(\beta_{0}, \boldsymbol{\gamma}_{0}\right) \pi\left(\beta_{1}, \boldsymbol{\gamma}_{1}\right) \pi\left(\boldsymbol{\beta}_{2}, \boldsymbol{\gamma}_{2}\right) \\
& \left.\times \prod_{j=1}^{J_{0}} \frac{1}{\lambda_{0 j}} \prod_{j=1}^{J_{1}} \frac{1}{\lambda_{1 j}} \prod_{j=1}^{J_{2}} \frac{1}{\lambda_{2 j}}\left[\frac{\exp \left\{-\frac{\varsigma \theta}{\theta}\right\}}{\theta^{\kappa_{\theta}+1}}\right]\left[\frac{\exp \left\{-\frac{\varsigma_{B}}{B}\right\}}{B^{\kappa_{B}+1}}\right] d \beta_{0} d \boldsymbol{\gamma}_{0} b \beta_{1} d \boldsymbol{\gamma}_{1} d \boldsymbol{\beta}_{2} d \boldsymbol{\gamma}_{2} d \boldsymbol{\lambda}_{\mathbf{0}} d \boldsymbol{\lambda}_{\mathbf{1}} d \boldsymbol{\lambda}_{\mathbf{2}} d \theta\right) \\
& \times d \boldsymbol{b} d B<\infty
\end{aligned}
$$

Para a verificação do item (i), são consideradas as condições (C1), (C2) e (C3) do Teorema 1 e seguindo Chen e Shao (2001), seja $F\left(K_{P i l}\right)=\frac{\mathrm{e}^{K_{\text {Pil }}}}{1+\mathrm{e}^{K_{\text {Pil }}}}$, com $K_{\text {Pil }}=$ $\boldsymbol{x}_{E i l}^{\top} \boldsymbol{\alpha}, \boldsymbol{x}_{E i l}=\left(1, A_{i l}, \boldsymbol{x}_{i l}^{\top}\right)^{\top}, \forall l$ e $\boldsymbol{\alpha}=\left(\alpha_{0}, \alpha_{1}, \boldsymbol{\alpha}_{2}^{\top}\right)^{\top}$ de modo que

$$
L_{a}\left(\boldsymbol{\alpha} \mid D_{o b s}\right)=\prod_{i \in \cup_{m=1}^{3} \mathfrak{N}_{m}, \forall l}\left[F\left(K_{P i l}\right)\right]^{E_{i l}}\left[1-F\left(K_{P i l}\right)\right]^{1-E_{i l}}
$$

Como $1-F(x)=\int_{-\infty}^{\infty} \mathbb{I}\{u>x\} d F(u)$, então vale que $F(x)=\int_{-\infty}^{\infty} \mathbb{I}\{u \leq x\} d F(u)$. Fazendo uma mudança de variável $u=-v$ com $d F(u)=d(-F(-v))$ tem-se que

$F(x)=\int_{-\infty}^{\infty} \mathbb{I}\{u \leq x\} d F(u)=\int_{-\infty}^{\infty} \mathbb{I}\{-v \leq x\} d(-F(-v))=\int_{-\infty}^{\infty} \mathbb{I}\{v \geq-x\} d(-F(-v))$.

Agora, relembrando que nas especificações anteriores ao teorema 1 , foi definido $w_{i l}=$ $\begin{cases}-1, & \text { se } E_{i l}=1, \\ 1, & \text { se } E_{i l}=0,\end{cases}$

- Para $w_{i l}=-1$ :

$$
\int_{-\infty}^{\infty} \mathbb{I}\left\{u_{i l}>-K_{P i l}\right\} d\left(-F\left(-u_{i l}\right)\right) \leq \int_{-\infty}^{\infty} \mathbb{I}\left\{u_{i l} \geq-K_{P i l}\right\} d\left(-F\left(-u_{i l}\right)\right)=F\left(K_{P i l}\right) .
$$

- Para $w_{i l}=1$ :

$$
\begin{gathered}
\int_{-\infty}^{\infty} \mathbb{I}\left\{u_{i l}>K_{\text {Pil }}\right\} d\left(F\left(u_{i l}\right)\right) \leq \int_{-\infty}^{\infty} \mathbb{I}\left\{u_{i l} \geq K_{\text {Pi }}\right\} d\left(F\left(u_{i l}\right)\right) \quad \text { implicaque } \\
1-F\left(K_{\text {Pil }}\right) \leq \int_{-\infty}^{\infty} \mathbb{I}\left\{u_{i l} \geq K_{\text {Pil }}\right\} d\left(F\left(u_{i l}\right)\right)
\end{gathered}
$$

Dessa forma, pode-se concluir que

$$
\begin{aligned}
\int_{-\infty}^{\infty} \mathbb{I}\left\{u_{i l}>-K_{P i l}\right\} d\left(-F\left(-u_{i l}\right)\right) & \leq\left[F\left(K_{P i l}\right)\right]^{E_{i l}}\left[1-F\left(K_{P i l}\right)\right]^{1-E_{i l}} \\
& \left.\leq \int_{-\infty}^{\infty} \mathbb{I}\left\{u_{i l} \geq K_{P i l}\right\} d F\left(u_{i l}\right)\right)
\end{aligned}
$$


ou seja,

$$
\begin{aligned}
\int_{-\infty}^{\infty} \mathbb{I}\left\{u_{i l}>w_{i l} K_{P i l}\right\} d\left(w_{i l} F\left(w_{i l} u_{i l}\right)\right) & \leq\left[F\left(K_{P i l}\right)\right]^{E_{i l}}\left[1-F\left(K_{P i l}\right)\right]^{1-E_{i l}} \\
& \leq \int_{-\infty}^{\infty} \mathbb{I}\left\{u_{i l} \geq w_{i l} K_{P i l}\right\} d\left(w_{i l} F\left(w_{i l} u_{i l}\right)\right) .
\end{aligned}
$$

Agora, pelo teorema de Fubini e denotando $d \boldsymbol{F}(\boldsymbol{u})$ como $d\left(w_{1 l} F\left(w_{1 l} u_{1 l}\right)\right), \ldots, d\left(w_{\text {pal }}\right.$ $\left.F\left(w_{\text {pal }} u_{\text {pal }}\right)\right)$, por (B.3) tem-se

$$
\begin{gathered}
\int_{\Omega^{n}} \int_{\Omega^{\boldsymbol{\alpha}}} \mathbb{I}\left\{u_{i l}>w_{i l} \boldsymbol{x}_{E i l}^{\top} \boldsymbol{\alpha}, 1 \leq i \leq n_{1}+n_{2}+n_{3}\right\} d(\boldsymbol{\alpha}) d \boldsymbol{F}(\boldsymbol{u}) \leq \int_{\Omega^{\boldsymbol{\alpha}}} \mathrm{L}_{a}\left(\boldsymbol{\alpha} \mid D_{o b s}\right) \pi(\boldsymbol{\alpha}) d(\boldsymbol{\alpha}) \\
\leq \int_{\Omega^{n}} \int_{\Omega^{\boldsymbol{\alpha}}} \mathbb{I}\left\{u_{i l} \geq w_{i l} \boldsymbol{x}_{E i l}^{\top} \boldsymbol{\alpha}, 1 \leq i \leq n_{1}+n_{2}+n_{3}\right\} d(\boldsymbol{\alpha}) d \boldsymbol{F}(\boldsymbol{u}) .
\end{gathered}
$$

Deste modo, pode-se obter

$$
\begin{aligned}
\int_{\Omega^{\boldsymbol{\alpha}}} \mathrm{L}_{a}\left(\boldsymbol{\alpha} \mid D_{o b s}\right) \pi(\boldsymbol{\alpha}) d(\boldsymbol{\alpha}) & =\int_{\Omega^{n}} \int_{\Omega^{\boldsymbol{\alpha}}} \mathbb{I}\left\{u_{i l}>w_{i l} \boldsymbol{x}_{E i l}^{\top} \boldsymbol{\alpha}, 1 \leq i \leq n_{1}+n_{2}+n_{3}\right\} d(\boldsymbol{\alpha}) d \boldsymbol{F}(\boldsymbol{u}) \\
& =\int_{\Omega^{n}} \int_{\Omega^{\boldsymbol{\alpha}}} \mathbb{I}\left\{\boldsymbol{X}_{E i}^{* \top} \boldsymbol{\alpha} \leq \boldsymbol{u}\right\} d(\boldsymbol{\alpha}) d \boldsymbol{F}(\boldsymbol{u}) .
\end{aligned}
$$

Considere-se aqui o Lema 4.1 de Chen e Shao (2001): Assuma satisfeitas as condições (C1) e (C2). Então, existe uma constante $k$ dependendo só de $\boldsymbol{X}_{\text {Eil }}^{*}$ tal que $\|\boldsymbol{\alpha}\| \leq k\|\boldsymbol{u}\|$, sempre que $\boldsymbol{X}_{\text {Eil }}^{* \top} \boldsymbol{\alpha} \leq \boldsymbol{u}$.

Agora, sendo $\|\cdot\|$ a norma 1, dada por $\|x\|=\|x\|_{1}=\sum_{i=1}^{n}\left|x_{i}\right|$, pelo lema tem-se que $\int_{\Omega^{\boldsymbol{\alpha}}} \mathbb{I}\left\{\boldsymbol{X}_{E i l}^{* \top} \boldsymbol{\alpha} \leq \boldsymbol{u}\right\} d(\boldsymbol{\alpha})=\int_{\left\{\boldsymbol{X}_{E i l}^{* \top} \boldsymbol{\alpha} \leq \boldsymbol{u}\right\}} d \boldsymbol{\alpha} \leq k\|\boldsymbol{u}\|^{k}$.

Finalmente, pela condição (C3), segue que $\int_{\Omega^{\alpha}} \mathrm{L}_{a}\left(\boldsymbol{\alpha} \mid D_{\text {obs }}\right) \pi(\boldsymbol{\alpha}) d(\boldsymbol{\alpha})<\infty$.

Para a prova do item (ii), o integrando é separado apropriadamente em duas partes segundo o parâmetro da dependência, $\theta$. Para isto, sejam

$$
L_{1 i l}\left(\beta_{0}, \gamma_{0}, \boldsymbol{\lambda}_{0} \mid D_{i l}, b_{l}\right)=\prod_{i \in \mathcal{N}_{1}, \forall l} h_{0}\left(y_{i l} \mid \boldsymbol{\lambda}_{0}\right) \mathrm{e}^{K_{D i l}+b_{l}} \exp \left\{-H_{0}\left(y_{i l} \mid \boldsymbol{\lambda}_{0}\right) \mathrm{e}^{K_{D i l}+b_{l}}\right\}
$$

$$
\begin{aligned}
& L_{2 i l}\left(\beta_{1}, \boldsymbol{\gamma}_{1}, \boldsymbol{\lambda}_{1}, \boldsymbol{\beta}_{2}, \boldsymbol{\gamma}_{2}, \boldsymbol{\lambda}_{2}, \theta \mid D_{i l}, b_{l}\right)=\prod_{i \in \mathcal{N}_{2}, \forall l} \\
& \frac{(1+\theta) h_{1}\left(y_{E i l} \mid \boldsymbol{\lambda}_{1}\right) \mathrm{e}^{K_{E i l}+b_{l}} h_{2}\left(y_{G i l} \mid \boldsymbol{\lambda}_{2}\right) \mathrm{e}^{K_{G i l}+b_{l}} \exp \left\{\theta H_{1}\left(y_{E i l} \mid \boldsymbol{\lambda}_{1}\right) \mathrm{e}^{K_{E i l}+b_{l}}+\theta H_{2}\left(y_{G i l} \mid \boldsymbol{\lambda}_{2}\right) \mathrm{e}^{K_{G i l}+b_{l}}\right\}}{\left[\exp \left\{\theta H_{1}\left(y_{E i l} \mid \boldsymbol{\lambda}_{1}\right) \mathrm{e}^{K_{E i l}+b_{l}}\right\}+\exp \left\{\theta H_{2}\left(y_{G i l} \mid \boldsymbol{\lambda}_{2}\right) \mathrm{e}^{K_{G i l}+b_{l}}\right\}-1\right]^{\frac{1}{\theta}+2}}
\end{aligned}
$$


$e$

$$
L_{3 i l}^{*}\left(\beta_{1}, \boldsymbol{\gamma}_{1}, \boldsymbol{\lambda}_{1} \mid D_{i l}, b_{l}\right)=\prod_{i \in \mathfrak{N}_{3}, \forall l} \frac{h_{1}\left(y_{E i l} \mid \boldsymbol{\lambda}_{1}\right) \mathrm{e}^{K_{E i l}+b_{l}}}{\exp \left\{H_{1}\left(y_{E i l} \mid \boldsymbol{\lambda}_{1}\right) \mathrm{e}^{K_{E i l}+b_{l}}\right\}} .
$$

Observe que $L_{3 i l}^{*}$ comparada com $L_{3 i l}$ já não é mais função dos parâmetros $\boldsymbol{\beta}_{2}, \boldsymbol{\gamma}_{2}, \boldsymbol{\lambda}_{2}, \theta$. Assim, a integral do item (ii) pode ser reescrita como

$$
\begin{gathered}
\int_{\Omega^{B}} \int_{\Omega^{\boldsymbol{b}}}\left(\int _ { \Omega ^ { \boldsymbol { \xi } } - \{ \boldsymbol { \alpha } \} } \left(\prod_{l=1}^{L} \prod_{i=1}^{n_{l}} L_{1 i l}\left(\beta_{0}, \boldsymbol{\gamma}_{0}, \boldsymbol{\lambda}_{0} \mid D_{i l}, b_{l}\right) L_{2 i l}\left(\beta_{1}, \boldsymbol{\gamma}_{1}, \boldsymbol{\lambda}_{1}, \boldsymbol{\beta}_{2}, \boldsymbol{\gamma}_{2}, \boldsymbol{\lambda}_{2}, \theta \mid D_{i l}, b_{l}\right)\right.\right. \\
\left.L_{3 i l}^{*}\left(\beta_{1}, \boldsymbol{\gamma}_{1}, \boldsymbol{\lambda}_{1} \mid D_{i l}, b_{l}\right)(2 \pi B)^{-\frac{1}{2}} \exp \left\{-\frac{b_{l}^{2}}{2 B}\right\}\right) \pi\left(\beta_{0}, \boldsymbol{\gamma}_{0}\right) \pi\left(\beta_{1}, \boldsymbol{\gamma}_{1}\right) \pi\left(\boldsymbol{\beta}_{2}, \boldsymbol{\gamma}_{2}\right) \prod_{j=1}^{J_{0}} \frac{1}{\lambda_{0 j}} \prod_{j=1}^{J_{1}} \frac{1}{\lambda_{1 j}} \\
\left.\prod_{j=1}^{J_{2}} \frac{1}{\lambda_{2 j}}\left[\frac{\exp \left\{-\frac{\varsigma_{\theta}}{\theta}\right\}}{\theta^{\kappa}+1}\right]\left[\frac{\exp \left\{-\frac{\varsigma_{B}}{B}\right\}}{B^{\kappa_{B}+1}}\right] d \beta_{0} d \boldsymbol{\gamma}_{0} b \beta_{1} d \boldsymbol{\gamma}_{1} d \boldsymbol{\beta}_{2} d \boldsymbol{\gamma}_{2} d \boldsymbol{\lambda}_{\mathbf{0}} d \boldsymbol{\lambda}_{\mathbf{1}} d \boldsymbol{\lambda}_{\mathbf{2}} d \theta\right) d \boldsymbol{b} d B .
\end{gathered}
$$

Então, será provado que esta expressão é finita, o que verifica o item (ii). Note que em relação aos parâmetros $\beta_{0}, \boldsymbol{\gamma}_{0}, \beta_{1}, \boldsymbol{\gamma}_{1}, \boldsymbol{\beta}_{2}, \boldsymbol{\gamma}_{2}, \boldsymbol{\lambda}_{\mathbf{0}}, \boldsymbol{\lambda}_{\mathbf{1}}, \boldsymbol{\lambda}_{\mathbf{2}}$ e $\theta$, pode-se resolver a integral interna em duas partes e provar que

(ii)a. $\int L_{1 i l}\left(\beta_{0}, \boldsymbol{\gamma}_{0}, \boldsymbol{\lambda}_{0} \mid D_{i l}, b_{l}\right) \pi\left(\beta_{0}, \gamma_{0}\right) \pi\left(\boldsymbol{\lambda}_{0}\right) d \beta_{0} d \boldsymbol{\gamma}_{0}<\infty e$

(ii)b. $\int L_{2 i l}\left(\beta_{1}, \boldsymbol{\gamma}_{1}, \boldsymbol{\lambda}_{1}, \boldsymbol{\beta}_{2}, \boldsymbol{\gamma}_{2}, \boldsymbol{\lambda}_{2}, \theta \mid D_{i l}, b_{l}\right) L_{3 i l}^{*}\left(\beta_{1}, \boldsymbol{\gamma}_{1}, \boldsymbol{\lambda}_{1}, \boldsymbol{\beta}_{2}, \boldsymbol{\gamma}_{2}, \boldsymbol{\lambda}_{2}, \theta \mid D_{i l}, b_{l}\right) \pi\left(\beta_{1}, \boldsymbol{\gamma}_{1}\right)$

$$
\pi\left(\boldsymbol{\beta}_{2}, \boldsymbol{\gamma}_{2}\right) \pi\left(\boldsymbol{\lambda}_{1}\right) \pi\left(\boldsymbol{\lambda}_{2}\right) \pi(\theta) d \beta_{1} d \boldsymbol{\gamma}_{1} d \boldsymbol{\beta}_{2} d \boldsymbol{\gamma}_{2} d \boldsymbol{\lambda}_{\mathbf{2}} d \theta<\infty
$$

Como será mostrado a seguir, ambas as funções nas integrais (ii)a. e (ii)b. são limitadas por cotas cuja integral em relação aos parâmetros não dependem do efeito aleatório, de tal forma que, para provar a finitude de (B.4) basta provar que

$$
\begin{aligned}
& \int_{\Omega^{B}} \int_{\Omega^{\boldsymbol{b}}}\left(\int _ { \Omega ^ { \boldsymbol { \xi } } - \{ \boldsymbol { \alpha } \} } \left(\prod_{i=1, \forall l}^{N} L_{1 i l}\left(\beta_{0}, \boldsymbol{\gamma}_{0}, \boldsymbol{\lambda}_{0} \mid D_{i l}, b_{l}\right) L_{2 i l}\left(\beta_{1}, \boldsymbol{\gamma}_{1}, \boldsymbol{\lambda}_{1}, \boldsymbol{\beta}_{2}, \boldsymbol{\gamma}_{2}, \boldsymbol{\lambda}_{2}, \theta \mid D_{i l}, b_{l}\right)\right.\right. \\
& \left.L_{3 i l}^{*}\left(\beta_{1}, \boldsymbol{\gamma}_{1}, \boldsymbol{\lambda}_{1} \mid D_{i l}, b_{l}\right)\right) \pi\left(\beta_{0}, \boldsymbol{\gamma}_{0}\right) \pi\left(\beta_{1}, \boldsymbol{\gamma}_{1}\right) \pi\left(\boldsymbol{\beta}_{2}, \boldsymbol{\gamma}_{2}\right) \prod_{j=1}^{J_{0}} \frac{1}{\lambda_{0 j}} \prod_{j=1}^{J_{1}} \frac{1}{\lambda_{1 j}} \prod_{j=1}^{J_{2}} \frac{1}{\lambda_{2 j}}\left[\frac{\exp \left\{-\frac{\varsigma_{\theta}}{\theta}\right\}}{\theta^{\kappa_{\theta}+1}}\right] \\
& \left.d \beta_{0} d \boldsymbol{\gamma}_{0} b \beta_{1} d \boldsymbol{\gamma}_{1} d \boldsymbol{\beta}_{2} d \boldsymbol{\gamma}_{2} d \boldsymbol{\lambda}_{\mathbf{0}} d \boldsymbol{\lambda}_{\mathbf{1}} d \boldsymbol{\lambda}_{\mathbf{2}} d \theta\right) \prod_{l=1}^{L}(2 \pi B)^{-\frac{1}{2}} \exp \left\{-\frac{b_{l}^{2}}{2 B}\right\} d b_{l}\left[\frac{\exp \left\{-\frac{\varsigma_{B}}{B}\right\}}{B^{\kappa_{B}+1}}\right] d B<\infty
\end{aligned}
$$

que pode ser escrita como

$$
\begin{aligned}
& \int_{\Omega^{\boldsymbol{\xi}-\{\boldsymbol{\alpha}\}}}\left(\prod_{i=1, \forall l}^{N} L_{1 i l}\left(\beta_{0}, \boldsymbol{\gamma}_{0}, \boldsymbol{\lambda}_{0} \mid D_{i l}, b_{l}\right) L_{2 i l}\left(\beta_{1}, \boldsymbol{\gamma}_{1}, \boldsymbol{\lambda}_{1}, \boldsymbol{\beta}_{2}, \boldsymbol{\gamma}_{2}, \boldsymbol{\lambda}_{2}, \theta \mid D_{i l}, b_{l}\right)\right. \\
& \left.\times L_{3 i l}^{*}\left(\beta_{1}, \boldsymbol{\gamma}_{1}, \boldsymbol{\lambda}_{1} \mid D_{i l}, b_{l}\right)\right) \pi\left(\beta_{0}, \boldsymbol{\gamma}_{0}\right) \pi\left(\beta_{1}, \boldsymbol{\gamma}_{1}\right) \pi\left(\boldsymbol{\beta}_{2}, \boldsymbol{\gamma}_{2}\right) \prod_{j=1}^{J_{0}} \frac{1}{\lambda_{0 j}} \prod_{j=1}^{J_{1}} \frac{1}{\lambda_{1 j}} \prod_{j=1}^{J_{2}} \frac{1}{\lambda_{2 j}}\left[\frac{\exp \left\{-\frac{\varsigma_{\theta}}{\theta}\right\}}{\theta^{\kappa_{\theta}+1}}\right] d \beta_{0} d \boldsymbol{\gamma}_{0}
\end{aligned}
$$




$$
\times d \beta_{1} d \boldsymbol{\gamma}_{1} d \boldsymbol{\beta}_{2} d \boldsymbol{\gamma}_{2} d \boldsymbol{\lambda}_{\mathbf{0}} d \boldsymbol{\lambda}_{\mathbf{1}} d \boldsymbol{\lambda}_{\mathbf{2}} d \theta \int_{\Omega^{b}} \prod_{l=1}^{L}(2 \pi B)^{-\frac{1}{2}} \exp \left\{-\frac{b_{l}^{2}}{2 B}\right\} d b_{l} \int_{\Omega^{B}}\left[\frac{\exp \left\{-\frac{\varsigma_{B}}{B}\right\}}{B^{\kappa_{B}+1}}\right] d B<\infty,
$$

e daqui observa-se que o valor das últimas duas integrais é 1.

$\underline{\text { Resolução de (ii)a. }}$

Vale relembrar aqui que o preditor linear dos efeitos fixos associado ao evento final é $K_{D i l}=A_{i l} \beta_{0}+\boldsymbol{x}_{i l}^{\top} \gamma_{0}$. Assim, tem-se que

$$
\begin{aligned}
& \int L_{1 i l}\left(\beta_{0}, \boldsymbol{\gamma}_{0}, \boldsymbol{\lambda}_{0} \mid D_{i l}, b_{l}\right) \pi\left(\beta_{0}, \boldsymbol{\gamma}_{0}\right) \pi\left(\boldsymbol{\lambda}_{0}\right) d \beta_{0} d \boldsymbol{\gamma}_{0} d \boldsymbol{\lambda}_{0}= \\
& \qquad \int \prod_{i \in \mathfrak{N}(, \forall l} \frac{h_{0}\left(y_{i l} \mid \boldsymbol{\lambda}_{0}\right) \mathrm{e}^{K_{D i l}+b_{l}}}{\exp \left\{H_{0}\left(y_{i l} \mid \boldsymbol{\lambda}_{0}\right) \mathrm{e}^{K_{D i l}+b_{l}}\right\}} \prod_{j=1}^{J_{0}} \frac{1}{\lambda_{0 j}} d \beta_{0} d \boldsymbol{\gamma}_{0} d \boldsymbol{\lambda}_{0}
\end{aligned}
$$

Em termos da função de risco basal exponencial por partes, a expressão anterior passa a ser

$$
\int \prod_{i \in \mathfrak{N}_{1}, \forall l} \prod_{j=1}^{J_{0}} \frac{\left(\lambda_{0 j} \mathrm{e}^{K_{D i l}+b_{l}}\right)^{\delta_{i l 0 j}}}{\exp \left\{\delta_{i l 0 j}\left[\lambda_{0 j}\left(y_{i l}-s_{0 j-1}\right)+\sum_{g=1}^{j-1} \lambda_{0 g}\left(s_{0 g}-s_{0 g-1}\right)\right] \mathrm{e}^{K_{D i l}+b_{l}}\right\}} \prod_{j=1}^{J_{0}} \frac{1}{\lambda_{0 j}} d \beta_{0} d \boldsymbol{\gamma}_{0} d \boldsymbol{\lambda}_{0}
$$

em que

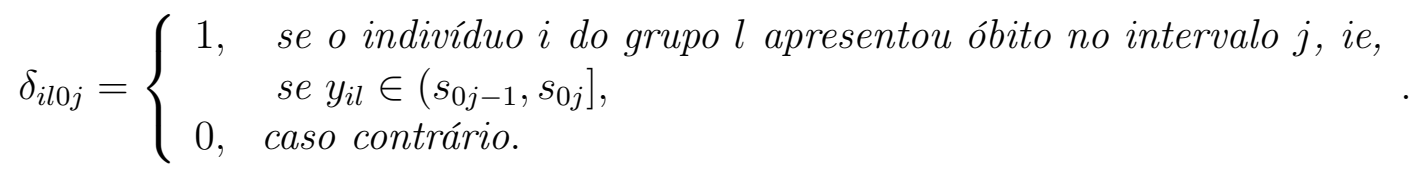

Agora, seja $j_{i}$ o indice tal que $s_{0 j_{i}-1}<y_{i} \leq s_{0 j_{i}}$, então, $\delta_{i l 0 j}= \begin{cases}1, & \text { se } j=j_{i}, \\ 0, & \text { se } j \neq j_{i},\end{cases}$ Com isto, note que a integral acima é limitada superiormente por

$$
\int \prod_{i \in \mathcal{N}_{1}, \forall l} \frac{\lambda_{0 j_{i}} \mathrm{e}^{K_{D i l}+b_{l}}}{\exp \left\{\lambda_{0 j_{i}}\left(y_{i l}-s_{0 j_{i}-1}\right) \mathrm{e}^{K_{D i l}+b_{l}}\right\}} \prod_{j=1}^{J_{0}} \frac{1}{\lambda_{0 j}} d \beta_{0} d \boldsymbol{\gamma}_{0} d \boldsymbol{\lambda}_{0}
$$

Seja $g(z)=z \exp \left\{-\left(y_{i l}-s_{0 j_{i}-1}\right) z\right\}$ em que $g:[0, \infty) \rightarrow \mathbb{R}$ é uma função contínua com $\lim _{z \rightarrow \infty} g(z)=0$. Assim, o teorema de Weierstrass garante que g é limitada $\forall z$, pois como $\lim _{z \rightarrow \infty} g(z)=0$, para cada $\epsilon>0$ existe $M \in \mathbb{N}$ tal que se $z>M$ então $|g(z)|<\epsilon$. Isto implica que, após $M, g$ é limitada por $\epsilon$, antes de $M, g$ é contínua no compacto $[0, M]$, logo g é limitada, ou seja, existe uma constante $K>0$ tal que $|g(z)|<K$ $\forall z \in[0, M]$, agora $|g(z)|<\max \{\epsilon, K\} \forall z \in[0, \infty)$. Portanto, existe uma constante 
$M_{1}>0$ tal que

$$
\frac{\lambda_{0 j_{i}} \mathrm{e}^{K_{D i l}+b_{l}}}{\exp \left\{\lambda_{0 j_{i}}\left(y_{i l}-s_{0 j_{i}-1}\right) \mathrm{e}^{K_{D i l}+b_{l}}\right\}} \leq M_{1}
$$

Considere a transformação $\varsigma_{0 j}=\ln \lambda_{0 j}, j=1, \cdots, J_{0}, e \varsigma_{0}=\left(\varsigma_{01}, \cdots, \varsigma_{0 J_{0}}\right)^{\top}$. Entãa $\lambda_{0 j} d \varsigma_{0 j}=d \lambda_{0 j} e$

$$
\begin{aligned}
\mathrm{L}_{1 i l}^{*}\left(\beta_{0}, \gamma_{0}, \varsigma_{0} \mid D_{i l}, b_{l}\right) & =L_{1 i l}\left(\beta_{0}, \gamma_{0}, \boldsymbol{\lambda}_{0}=\mathrm{e}^{\varsigma_{0}} \mid D_{i l}, b_{l}\right)\left|\frac{\partial\left(\lambda_{01}, \cdots \lambda_{J_{0}}\right)}{\partial\left(\varsigma_{01}, \cdots, \varsigma_{J_{0}}\right)}\right| \\
& =L_{1 i l}\left(\beta_{0}, \gamma_{0}, \mathrm{e}^{\varsigma_{0}} \mid D_{i l}, b_{l}\right) \prod_{j=1}^{J_{0}} \lambda_{0 j} .
\end{aligned}
$$

Portanto,

$$
\prod_{i \in \mathcal{N}(, \forall l} \frac{\lambda_{0 j_{i}} \mathrm{e}^{K_{D i l}+b_{l}}}{\exp \left\{\lambda_{0 j_{i}}\left(y_{i l}-s_{0 j_{i}-1}\right) \mathrm{e}^{K_{D i l}+b_{l}}\right\}} \prod_{j=1}^{J_{0}} \frac{1}{\lambda_{0 j}} \leq \prod_{i \in \mathcal{N}_{(}, \forall l} \frac{\mathrm{e}^{\varsigma_{0 j_{i}}+K_{D_{i l}}+b_{l}}}{\exp \left\{\left(y_{i l}-s_{0 j_{i}-1}\right) \mathrm{e}^{\varsigma_{0 j_{i}}+K_{D_{i l}}+b_{l}}\right\}} .
$$

Pela condição (C3) em que $\boldsymbol{X}_{0}$ é de posto completo $J_{0}+p_{0}$, existem $J_{0}+p_{0}$ distintos indices $i_{1}, \cdots, i_{J_{0}+p_{0}} \in \mathcal{N}_{1}$ tais que a matriz $\boldsymbol{X}_{0}^{*}$ de ordem $\left(J_{0}+p_{0}\right) \times\left(J_{0}+p_{0}\right)$ formada pelas linhas $\left(\cdots, \delta_{0 j_{i_{t}}}, \cdots, A_{i l_{t}}, \boldsymbol{x}_{i l_{t}}^{\top}\right)$, para $t=1, \cdots, J_{0}+P_{0}$, é de posto completo. Logo, usando (B.5) e (B.6) e com $M_{2}>0$ constante, tem-se

$$
\begin{aligned}
& \prod_{i \in \mathcal{N}_{1}, \forall l} \frac{h_{0}\left(y_{i l} \mid \boldsymbol{\lambda}_{0}\right) \mathrm{e}^{K_{D i l}+b_{l}}}{\exp \left\{H_{0}\left(y_{i l} \mid \boldsymbol{\lambda}_{0}\right) \mathrm{e}^{K_{D i l}+b_{l}}\right\}} \prod_{j=1}^{J_{0}} \frac{1}{\lambda_{0 j}}
\end{aligned}
$$

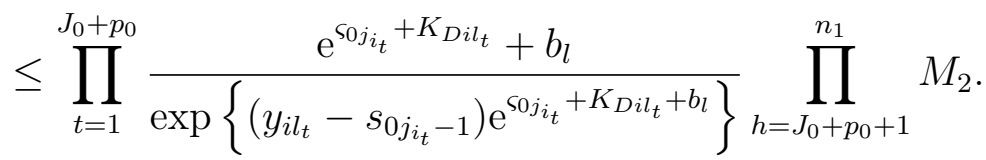

Agora, considerando a transformação um a um

$$
\begin{gathered}
\boldsymbol{\varphi}_{0}=\left[\begin{array}{c}
\varphi_{01} \\
\vdots \\
\varphi_{0, J_{0}+P_{0}}
\end{array}\right]=\boldsymbol{X}_{0}^{*}\left[\begin{array}{c}
\boldsymbol{\varsigma}_{0}^{\top} \\
\beta_{0} \\
\boldsymbol{\gamma}_{0}^{\top}
\end{array}\right]=\left[\begin{array}{c}
\varsigma_{01}+A_{1} \beta_{0}+\boldsymbol{X}_{1}^{\top} \boldsymbol{\gamma}_{0} \\
\vdots \\
\varsigma_{0, J_{0}+P_{0}}+A_{J_{0}+P_{0}} \beta_{0}+\boldsymbol{x}_{J_{0}+P_{0}}^{\top} \boldsymbol{\gamma}_{0}
\end{array}\right] \text { temos } \\
\prod_{i \in \mathcal{N}_{1}, \forall l} \frac{h_{0}\left(y_{i l} \mid \boldsymbol{\lambda}_{0}\right) \mathrm{e}^{K_{D i l}+b_{l}}}{\exp \left\{H_{0}\left(y_{i l} \mid \boldsymbol{\lambda}_{0}\right) \mathrm{e}^{\left.K_{D i l}+b_{l}\right\}}\right.} \prod_{j=1}^{J_{0}} \frac{1}{\lambda_{0 j}} \leq M_{3} \prod_{t=1}^{J_{0}+P_{0}} \frac{\mathrm{e}^{\varphi_{0 t}+b_{l}}}{\exp \left\{\mathrm{e}^{\varphi_{0 t}+b_{l}}\left(y_{i l_{t}}-s_{0 j_{i_{t}}-1}\right)\right\}},
\end{gathered}
$$

em que $M_{3}>0$ é constante. Logo, segue que 


$$
\int \prod_{i \in \mathfrak{N}_{1}, \forall l} \frac{h_{0}\left(y_{i l} \mid \boldsymbol{\lambda}_{0}\right) \mathrm{e}^{K_{D i l}+b_{l}}}{\exp \left\{H_{0}\left(y_{i l} \mid \boldsymbol{\lambda}_{0}\right) \mathrm{e}^{K_{D i l}+b_{l}}\right\}} \prod_{j=1}^{J_{0}} \frac{1}{\lambda_{0 j}} d \beta_{0} d \boldsymbol{\gamma}_{0} d \boldsymbol{\lambda}_{0} \leq M_{3} e
$$

$\int_{-\infty}^{\infty} \prod_{t=1}^{J_{0}+P_{0}} \frac{\mathrm{e}^{\varphi_{0_{t}}+b_{l}}}{\exp \left\{\mathrm{e}^{\varphi_{0_{t}}+b_{l}}\left(y_{i l_{t}}-s_{0 j_{i_{t}}-1}\right)\right\}} d \varphi_{0_{t}}=M_{3} \prod_{t=1}^{J_{0}+P_{0}} \int_{-\infty}^{\infty} \frac{\mathrm{e}^{\varphi_{0_{t}}+b_{l}}}{\exp \left\{\mathrm{e}^{\varphi_{0_{t}}+b_{l}}\left(y_{i l_{t}}-s_{0 j_{i_{t}}-1}\right)\right\}} d \varphi_{0_{t}}$.

Seja $u=\mathrm{e}^{\varphi_{0_{t}}+b_{l}}\left(y_{i l_{t}}-s_{0 j_{i_{t}}-1}\right)$, de modo que $d u=\mathrm{e}^{\varphi_{0_{t}}+b_{l}}\left(y_{i l_{t}}-s_{0 j_{i_{t}}-1}\right) d \varphi_{0_{l}}$. Então,

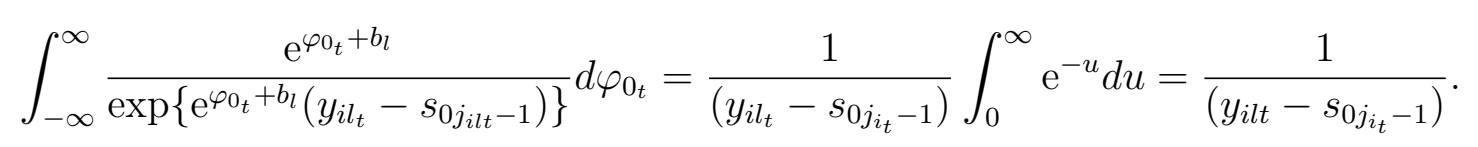

Segue que,

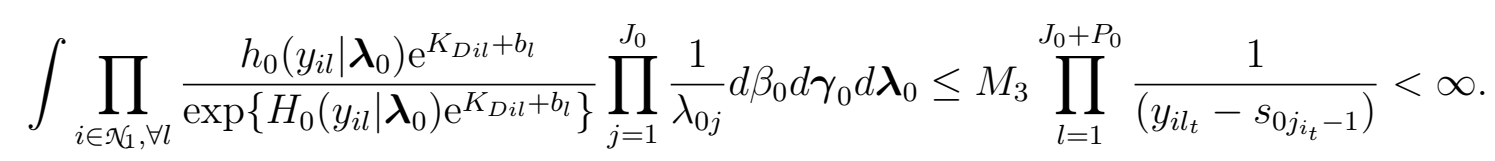

Note-se que aqui o efeito aleatório $b_{l}$ foi excluído naturalmente na resolução da integral.

\section{$\underline{\text { Resolução de (ii)b. }}$}

Neste caso, os preditores lineares dos efeitos fixos associado aos eventos de progressão e óbito após progressão são respetivamente $K_{\text {Eil }}=A_{i l} \beta_{1}+\boldsymbol{x}_{i l}^{\top} \gamma_{1}$ e $K_{G i l}=A_{i l} \beta_{21}+V_{i l}(1-$ $\left.A_{i l}\right) \beta_{i l}+\boldsymbol{z}_{i l}^{\top} \boldsymbol{\gamma}_{2}$. Logo a integral em (ii)b é

$$
\begin{aligned}
& \int L_{2 i l}\left(\beta_{1}, \boldsymbol{\gamma}_{1}, \boldsymbol{\lambda}_{1}, \boldsymbol{\beta}_{2}, \boldsymbol{\gamma}_{2}, \boldsymbol{\lambda}_{2}, \theta \mid D_{i l}, b_{l}\right) L_{3 i l}^{*}\left(\beta_{1}, \boldsymbol{\gamma}_{1}, \boldsymbol{\lambda}_{1}, \boldsymbol{\beta}_{2}, \boldsymbol{\gamma}_{2}, \boldsymbol{\lambda}_{2}, \theta \mid D_{i l}, b_{l}\right) \pi\left(\beta_{1}, \boldsymbol{\gamma}_{1}\right) \\
& \times \pi\left(\boldsymbol{\beta}_{2}, \boldsymbol{\gamma}_{2}\right) \pi\left(\boldsymbol{\lambda}_{1}\right) \pi\left(\boldsymbol{\lambda}_{2}\right) \pi(\theta) \pi(B) d \beta_{1} d \boldsymbol{\gamma}_{1} d \boldsymbol{\beta}_{2} d \boldsymbol{\gamma}_{2} d \boldsymbol{\lambda}_{\mathbf{2}} d \theta d B=\int \prod_{i \in \mathfrak{N}_{2}, \forall l}(1+\theta) \\
& \frac{h_{1}\left(y_{E i l} \mid \boldsymbol{\lambda}_{1}\right) \mathrm{e}^{K_{E i l}+b_{l}} h_{2}\left(y_{G i l} \mid \boldsymbol{\lambda}_{2}\right) \mathrm{e}^{K_{G i l}+b_{l}} \exp \left\{\theta H_{1}\left(y_{E i l} \mid \boldsymbol{\lambda}_{1}\right) \mathrm{e}^{K_{E i l}+b_{l}}+\theta H_{2}\left(y_{G i l} \mid \boldsymbol{\lambda}_{2}\right) \mathrm{e}^{K_{G i l}+b_{l}}\right\}}{\left[\exp \left\{\theta H_{1}\left(y_{E i l} \mid \boldsymbol{\lambda}_{1}\right) \mathrm{e}^{K_{E i l}+b_{l}}\right\}+\exp \left\{\theta H_{2}\left(y_{G i l} \mid \boldsymbol{\lambda}_{2}\right) \mathrm{e}^{K_{G i l}+b_{l}}\right\}-1\right]^{\frac{1}{\theta}+2}} \\
& \times \prod_{i \in \mathcal{N}_{(3}, \forall l} \frac{h_{1}\left(y_{E i l} \mid \boldsymbol{\lambda}_{1}\right) \mathrm{e}^{K_{E i l}+b_{l}}}{\exp \left\{H_{1}\left(y_{E i l} \mid \boldsymbol{\lambda}_{1}\right) \mathrm{e}^{K_{E i l}+b_{l}}\right\}} \prod_{j=1}^{J_{0}} \frac{1}{\lambda_{0 j}} \prod_{j=1}^{J_{1}} \frac{1}{\lambda_{1 j}} \prod_{j=1}^{J_{2}} \frac{1}{\lambda_{2 j}}\left[\frac{\exp \left\{-\frac{\varsigma \theta}{\theta}\right\}}{\theta^{\kappa_{\theta}+1}}\right]\left[\frac{\exp \left\{-\frac{\varsigma_{B}}{B}\right\}}{B^{\kappa_{B}+1}}\right] \\
& \times d \beta_{0} d \boldsymbol{\gamma}_{0} b \beta_{1} d \boldsymbol{\gamma}_{1} d \boldsymbol{\beta}_{2} d \boldsymbol{\gamma}_{2} d \boldsymbol{\lambda}_{\mathbf{0}} d \boldsymbol{\lambda}_{\mathbf{1}} d \boldsymbol{\lambda}_{\mathbf{2}} d \theta d B .
\end{aligned}
$$

Observe que a função verossimilhança $L_{2 i l}\left(\beta_{1}, \boldsymbol{\gamma}_{1}, \boldsymbol{\lambda}_{1}, \boldsymbol{\beta}_{2}, \boldsymbol{\gamma}_{2}, \boldsymbol{\lambda}_{2}, \theta \mid D_{i l}, b_{l}\right)$ pode ser interpretada de forma condicional dado que $T_{\text {Gil }}$ só acontece após observar $T_{\text {Eil }}$, e assim, pode ser reescrita como

$$
\begin{array}{r}
L_{2 i l}\left(\beta_{1}, \boldsymbol{\gamma}_{1}, \boldsymbol{\lambda}_{1}, \boldsymbol{\beta}_{2}, \boldsymbol{\gamma}_{2}, \boldsymbol{\lambda}_{2}, \theta \mid D_{i l}, b_{l}\right)= \\
L_{2 b}^{*}\left(\boldsymbol{\beta}_{2}, \boldsymbol{\gamma}_{2}, \boldsymbol{\lambda}_{2} \mid D_{i l}, b_{l}, \beta_{1}, \boldsymbol{\gamma}_{1}, \boldsymbol{\lambda}_{1}, \theta\right) L_{2 a}^{*}\left(\beta_{1}, \boldsymbol{\gamma}_{1}, \boldsymbol{\lambda}_{1}, \theta \mid D_{i l}, b_{l}\right) .
\end{array}
$$


Separando adequadamente $L_{2 i l}\left(\beta_{1}, \boldsymbol{\gamma}_{1}, \boldsymbol{\lambda}_{1}, \boldsymbol{\beta}_{2}, \boldsymbol{\gamma}_{2}, \boldsymbol{\lambda}_{2}, \theta \mid D_{i l}, b_{l}\right)$, tem-se

$$
\begin{aligned}
L_{2 i l}\left(\beta_{1}, \boldsymbol{\gamma}_{1}, \boldsymbol{\lambda}_{1}, \boldsymbol{\beta}_{2}, \boldsymbol{\gamma}_{2}, \boldsymbol{\lambda}_{2}, \theta \mid D_{i l}, b_{l}\right)= \\
\quad \prod_{i \in \mathfrak{N}_{2}, \forall l} \frac{(1+\theta) h_{2}\left(y_{G i l} \mid \boldsymbol{\lambda}_{2}\right) \mathrm{e}^{K_{G i l}+b_{l}} \exp \left\{\theta H_{2}\left(y_{G i l} \mid \boldsymbol{\lambda}_{2}\right) \mathrm{e}^{K_{G i l}+b_{l}}\right\}}{\left[\exp \left\{\theta H_{1}\left(y_{E i l} \mid \boldsymbol{\lambda}_{1}\right) \mathrm{e}^{K_{E i l}+b_{l}}\right\}+\exp \left\{\theta H_{2}\left(y_{G i l} \mid \boldsymbol{\lambda}_{2}\right) \mathrm{e}^{K_{G i l}+b_{l}}\right\}-1\right]^{\frac{1}{2 \theta}+1}} \\
\quad \times \prod_{i \in \mathfrak{N}_{2}, \forall l} \frac{h_{1}\left(y_{E i l} \mid \boldsymbol{\lambda}_{1}\right) \mathrm{e}^{K_{E i l}+b_{l}} \exp \left\{\theta H_{1}\left(y_{E i l} \mid \boldsymbol{\lambda}_{1}\right) \mathrm{e}^{K_{E i l}+b_{l}}\right\}}{\left[\exp \left\{\theta H_{1}\left(y_{E i l} \mid \boldsymbol{\lambda}_{1}\right) \mathrm{e}^{K_{E i l}+b_{l}}\right\}+\exp \left\{\theta H_{2}\left(y_{G i l} \mid \boldsymbol{\lambda}_{2}\right) \mathrm{e}^{K_{G i l}+b_{l}}\right\}-1\right]^{\frac{1}{2 \theta}+1}} .
\end{aligned}
$$

Considerando que $\theta H_{2}\left(y_{G i l} \mid \boldsymbol{\lambda}_{2}\right) \mathrm{e}^{K_{G i l}}>0$ e $\theta H_{1}\left(y_{E i l} \mid \boldsymbol{\lambda}_{1}\right) \mathrm{e}^{K_{E i}}>0$, então $\exp \left\{\theta H_{2}\left(y_{G i l} \mid \boldsymbol{\lambda}_{2}\right) \mathrm{e}^{K_{G i}}\right\} \geq 1 e \exp \left\{\theta H_{1}\left(y_{E i l} \mid \boldsymbol{\lambda}_{1}\right) \mathrm{e}^{K_{E i}}\right\} \geq 1$. Assim

$$
\begin{aligned}
L_{2 i l}\left(\beta_{1}, \boldsymbol{\gamma}_{1}, \boldsymbol{\lambda}_{1}, \boldsymbol{\beta}_{2}, \boldsymbol{\gamma}_{2}, \boldsymbol{\lambda}_{2}, \theta \mid D_{i l}, b_{l}\right) \leq \\
\quad \prod_{i \in \mathfrak{N}_{2}, \forall l} \frac{(1+\theta) h_{2}\left(y_{G i l} \mid \boldsymbol{\lambda}_{2}\right) \mathrm{e}^{K_{G i l}+b_{l}}}{\left[\exp \left\{\theta H_{1}\left(y_{E i l} \mid \boldsymbol{\lambda}_{1}\right) \mathrm{e}^{K_{E i l}+b_{l}}\right\}+\exp \left\{\theta H_{2}\left(y_{G i l} \mid \boldsymbol{\lambda}_{2}\right) \mathrm{e}^{K_{G i l}+b_{l}}\right\}-1\right]^{\frac{1}{2 \theta}}} \\
\quad \times \prod_{i \in \mathfrak{N}_{2}, \forall l} \frac{h_{1}\left(y_{E i l} \mid \boldsymbol{\lambda}_{1}\right) \mathrm{e}^{K_{E i l}+b_{l}}}{\left[\exp \left\{\theta H_{1}\left(y_{E i l} \mid \boldsymbol{\lambda}_{1}\right) \mathrm{e}^{K_{E i l}+b_{l}}\right\}+\exp \left\{\theta H_{2}\left(y_{G i l} \mid \boldsymbol{\lambda}_{2}\right) \mathrm{e}^{K_{G i l}+b_{l}}\right\}-1\right]^{\frac{1}{2 \theta}}} \\
\leq \prod_{i \in \mathfrak{N}_{2}, \forall l} \frac{(1+\theta) h_{2}\left(y_{G i l} \mid \boldsymbol{\lambda}_{2}\right) \mathrm{e}^{K_{G i l}+b_{l}}}{\left[\exp \left\{\theta H_{2}\left(y_{G i l} \mid \boldsymbol{\lambda}_{2}\right) \mathrm{e}^{K_{G i l}+b_{l}}\right\}\right]^{\frac{1}{2 \theta}}} \prod_{i \in \mathcal{N}_{2}, \forall l} \frac{h_{1}\left(y_{E i l} \mid \boldsymbol{\lambda}_{1}\right) \mathrm{e}^{K_{E i l}+b_{l}}}{\left[\exp \left\{\theta H_{1}\left(y_{E i l} \mid \boldsymbol{\lambda}_{1}\right) \mathrm{e}^{K_{E i l}+b_{l}}\right\}\right]^{\frac{1}{2 \theta}}} \\
\quad=\prod_{i \in \mathfrak{N}_{2}, \forall l} \frac{(1+\theta) h_{2}\left(y_{G i l} \mid \boldsymbol{\lambda}_{2}\right) \mathrm{e}^{K_{G i l}+b_{l}}}{\exp \left\{\frac{1}{2} H_{2}\left(y_{G i l} \mid \boldsymbol{\lambda}_{2}\right) \mathrm{e}^{K_{G i l}+b_{l}}\right\}} \prod_{i \in \mathcal{N}_{2}, \forall l} \frac{h_{1}\left(y_{E i l} \mid \boldsymbol{\lambda}_{1}\right) \mathrm{e}^{K_{E i l}+b_{l}}}{\exp \left\{\frac{1}{2} H_{1}\left(y_{E i l} \mid \boldsymbol{\lambda}_{1}\right) \mathrm{e}^{K_{E i l}+b_{l}}\right\}}
\end{aligned}
$$

Logo,

$$
\begin{array}{r}
L_{2 i l}\left(\beta_{1}, \boldsymbol{\gamma}_{1}, \boldsymbol{\lambda}_{1}, \boldsymbol{\beta}_{2}, \boldsymbol{\gamma}_{2}, \boldsymbol{\lambda}_{2}, \theta \mid D_{i l}, b_{l}\right) \leq \\
L_{2 b}^{*}\left(\boldsymbol{\beta}_{2}, \boldsymbol{\gamma}_{2}, \boldsymbol{\lambda}_{2} \mid D_{i l}, b_{l}, \beta_{1}, \boldsymbol{\gamma}_{1}, \boldsymbol{\lambda}_{1}, \theta\right) L_{2 a}^{*}\left(\beta_{1}, \boldsymbol{\gamma}_{1}, \boldsymbol{\lambda}_{1}, \theta \mid D_{i l}, b_{l}\right),
\end{array}
$$

em que

$$
L_{2 a}^{*}\left(\beta_{1}, \boldsymbol{\gamma}_{1}, \boldsymbol{\lambda}_{1}, \theta \mid D_{i l}, b_{l}\right)=\prod_{i \in \mathcal{N}_{2}, \forall l} \frac{h_{1}\left(y_{E i l} \mid \boldsymbol{\lambda}_{1}\right) \mathrm{e}^{K_{E i l}+b_{l}}}{\exp \left\{\frac{1}{2} H_{1}\left(y_{E i l} \mid \boldsymbol{\lambda}_{1}\right) \mathrm{e}^{K_{E i l}+b_{l}}\right\}}
$$

e

$$
L_{2 b}^{*}\left(\boldsymbol{\beta}_{2}, \boldsymbol{\gamma}_{2}, \boldsymbol{\lambda}_{2} \mid D_{i l}, b_{l}, \beta_{1}, \boldsymbol{\gamma}_{1}, \boldsymbol{\lambda}_{1}, \theta\right)=\prod_{i \in \mathcal{N}(2, \forall l} \frac{(1+\theta) h_{2}\left(y_{G i l} \mid \boldsymbol{\lambda}_{2}\right) \mathrm{e}^{K_{G i l}+b_{l}}}{\exp \left\{\frac{1}{2} H_{2}\left(y_{G i l} \mid \boldsymbol{\lambda}_{2}\right) \mathrm{e}^{K_{G i l}+b_{l}}\right\}}
$$

Note também que

$$
\begin{aligned}
& L_{3 i l}^{*}\left(\beta_{1}, \boldsymbol{\gamma}_{1}, \boldsymbol{\lambda}_{1} \mid D_{i l}, b_{l}\right)= \\
& \prod_{i \in \mathfrak{N}_{3}, \forall l} \frac{h_{1}\left(y_{E i l} \mid \boldsymbol{\lambda}_{1}\right) \mathrm{e}^{K_{E i l}+b_{l}}}{\exp \left\{H_{1}\left(y_{E i l} \mid \boldsymbol{\lambda}_{1}\right) \mathrm{e}^{K_{E i l}+b_{l}}\right\}} \leq \prod_{i \in \mathfrak{N}, \forall l} \frac{h_{1}\left(y_{E i l} \mid \boldsymbol{\lambda}_{1}\right) \mathrm{e}^{K_{E i l}+b_{l}}}{\exp \left\{\frac{1}{2} H_{1}\left(y_{E i l} \mid \boldsymbol{\lambda}_{1}\right) \mathrm{e}^{K_{E i l}+b_{l}}\right\}} .
\end{aligned}
$$

Assim, pela independência a priori assumida entre os parâmetros, pode-se obter uma 
cota superior para (B.7) da forma

$$
\begin{gathered}
\int \prod_{i \in \mathfrak{N}_{2}, \forall l} \frac{h_{2}\left(y_{G i l} \mid \boldsymbol{\lambda}_{2}\right) \mathrm{e}^{K_{G i l}+b_{l}}}{\exp \left\{\frac{1}{2} H_{2}\left(y_{G i l} \mid \boldsymbol{\lambda}_{2}\right) \mathrm{e}^{K_{G i l}+b_{l}}\right\}} \prod_{j=1}^{J_{2}} \frac{1}{\lambda_{2 j}} d \boldsymbol{\beta}_{2} d \boldsymbol{\gamma}_{2} d \boldsymbol{\lambda}_{2} \\
\times \int \prod_{i \in \mathfrak{N}_{2} \cup \mathfrak{N}_{3}, \forall l} \frac{h_{1}\left(y_{E i l} \mid \boldsymbol{\lambda}_{1}\right) \mathrm{e}^{K_{E i l}+b_{l}}}{\exp \left\{\frac{1}{2} H_{1}\left(y_{E i l} \mid \boldsymbol{\lambda}_{1}\right) \mathrm{e}^{K_{E i l}+b_{l}}\right\}} \prod_{j=1}^{J_{1}} \frac{1}{\lambda_{1 j}} d \beta_{1} d \boldsymbol{\gamma}_{1} d \boldsymbol{\lambda}_{1} \\
\times \int(1+\theta) \pi(\theta) d \theta .
\end{gathered}
$$

Como na prova em (ii) define-se para (B.8)

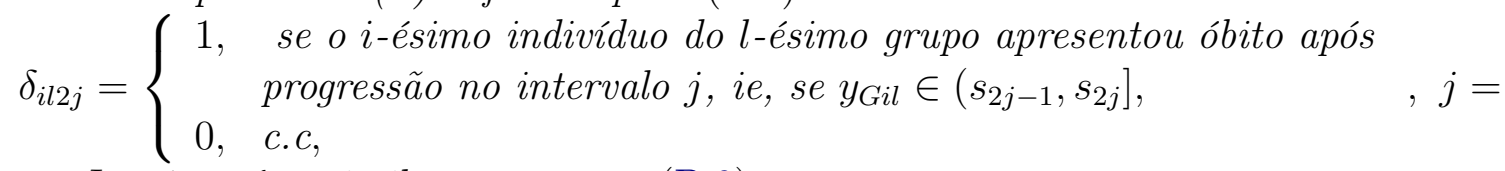
$1, \cdots, J_{2}$ e $i \in \mathcal{N}_{2}$ e similarmente para (B.9)

$\delta_{i l 1 j}=\left\{\begin{array}{l}1, \quad \text { se o i-ésimo indivíduo do l-ésimo grupo apresentou progressão }, \\ \text { no intervalo } j, \text { ie, se } y_{E i l} \in\left(s_{1 j-1}, s_{1 j}\right],\end{array}\right.$,

$j=1, \cdots, J_{1}$ e $i \in \mathcal{N}_{2} \cup \mathcal{N}_{3}$.

Assim, sendo G o integrando em (B.8), ele pode ser escrito como

$$
G=\prod_{i \in \mathcal{N}_{2}, \forall l} \prod_{j=1}^{J_{2}} \frac{\left(\lambda_{2 j} \mathrm{e}^{K_{G i l}+b_{l}}\right)^{\delta_{i l 2 j}}}{\exp \left\{\frac{1}{2} \delta_{i l 2 j}\left[\lambda_{2 j}\left(y_{G i l}-s_{2, j-1}\right)+\sum_{g=1}^{j-1} \lambda_{2 g}\left(s_{2, g}-s_{2, g-1}\right)\right] \mathrm{e}^{K_{G i l}+b_{l}}\right\}} \prod_{j=1}^{J_{2}} \frac{1}{\lambda_{2 j}} .
$$

Seja $j_{i}$ o indice tal que $s_{2, j_{i}-1}<y_{i} \leq s_{2, j_{i}}$. Então, $\delta_{i l 2 j}=\left\{\begin{array}{ll}1, & \text { se } j=j_{i}, \\ 0, & \text { se } j \neq j_{i},\end{array}, e\right.$

$$
\begin{aligned}
G & \leq \prod_{i \in \mathfrak{N}_{2}, \forall l} \frac{\lambda_{2 j_{i}} \mathrm{e}^{K_{G i l}+b_{l}}}{\exp \left\{\frac{1}{2}\left[\lambda_{2 j_{i}}\left(y_{G i l}-s_{2 j_{i}-1}\right)+\sum_{g=1}^{j_{i}-1} \lambda_{2 g}\left(s_{2, g}-s_{2, g-1}\right)\right] \mathrm{e}^{K_{G i l}+b_{l}}\right\}} \prod_{j=1}^{J_{2}} \frac{1}{\lambda_{2 j}} \\
& \leq \prod_{i \in \mathcal{N}_{2}} \frac{\lambda_{2 j_{i l}} \mathrm{e}^{K_{G i l}+b_{l}}}{\exp \left\{\frac{1}{2} \lambda_{2 j_{i}}\left(y_{G i l}-s_{2 j_{i}-1}\right) \mathrm{e}^{K_{G i l}+b_{l}}\right\}} \prod_{j=1}^{J_{2}} \frac{1}{\lambda_{2 j}} .
\end{aligned}
$$

Agora, dado que $\left(y_{G i l}-s_{2 j_{i l}-1}\right)>0$, como na prova do item anteior (ii)a, pelo teorema de Weierstrass, existe uma constante $M_{11}>0$ tal que

$$
\frac{\lambda_{2 j_{i}} \mathrm{e}^{K_{G i l}+b_{l}}}{\exp \left\{\frac{1}{2} \lambda_{2 j_{i}}\left(y_{G i l}-s_{2 j_{i}-1}\right) \mathrm{e}^{K_{G i l}+b_{l}}\right\}} \leq M_{11}
$$


Considerando a transformação $\varsigma_{2 j}=\ln \lambda_{2 j}, j=1, \cdots, J_{2}$, e $\boldsymbol{\varsigma}_{2}=\left(\varsigma_{21}, \cdots, \varsigma_{2 J_{2}}\right)^{\top}$, tem-se $\lambda_{2 j} d \varsigma_{2 j}=d \lambda_{2 j}$ e

$$
F \leq \prod_{i \in \mathfrak{N}_{2}, \forall l} \frac{\mathrm{e}^{\varsigma_{2 j_{i}}+K_{G_{i l}+b_{l}}}}{\exp \left\{\frac{1}{2}\left(y_{G i l}-s_{2 j_{i}-1}\right) \mathrm{e}^{\varsigma_{2 j_{i}}+K_{G_{i l}+b_{l}}}\right\}} .
$$

Pela condição (C3) em que $\boldsymbol{X}_{2}$ é de posto completo $J_{2}+p_{2}$, existem $J_{2}+p_{2}$ distintos indices $i_{1}, \cdots, i_{J_{2}+p_{2}} \in \mathcal{N}_{2}$ tais que a matriz $\boldsymbol{X}_{2}^{*}$ de ordem $\left(J_{2}+p_{2}\right) \times\left(J_{2}+p_{2}\right)$ com linhas $\left(\cdots, \delta_{2 j_{i}}, \cdots, A_{i l_{t}}, V_{i l_{t}}\left(1-A_{i l_{t}}\right), \boldsymbol{z}_{i l_{t}}^{\top}\right)$ para $t=1, \cdots, J_{2}+P_{2}$, é de posto completo. Logo, usando (B.11) e (B.12) e com $M_{12}$ uma constante, tem-se

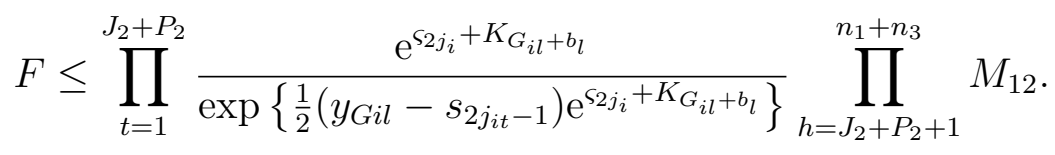

Logo, assumindo a transformação um a um,

$\boldsymbol{\varphi}_{2}=\left[\begin{array}{c}\varphi_{21} \\ \vdots \\ \varphi_{2, J_{2}+P_{2}}\end{array}\right]=\boldsymbol{X}_{2}^{*}\left[\begin{array}{c}\boldsymbol{\varsigma}_{2}^{\top} \\ \beta_{2} \\ \gamma_{2}^{\top}\end{array}\right]=\left[\begin{array}{c}\varsigma_{21}+A_{1} \beta_{21}+V_{1}\left(1-A_{1}\right) \beta_{22}+\boldsymbol{X}_{1}^{\top} \boldsymbol{\gamma}_{2} \\ \vdots \\ \varsigma_{2, J_{2}+P_{2}}+A_{J_{2}+P_{2}} \beta_{21}+V_{J_{2}+P_{2}}\left(1-A_{J_{2}+P_{2}}\right) \beta_{22}+\boldsymbol{x}_{J_{2}+P_{2}}^{\top} \gamma_{2}\end{array}\right]$,

obtém-se

$$
G \leq M_{13} \prod_{t=1}^{J_{2}+P_{2}} \frac{\mathrm{e}^{\varphi_{2}}}{\exp \left\{\frac{1}{2}\left(y_{G i l}-s_{2 j_{i_{t}}-1}\right) \mathrm{e}^{\varphi_{2_{t}}}\right\}}
$$

em que $M_{13}>0$ é uma constante. Logo, segue que

$$
\begin{aligned}
\int \prod_{i \in \mathcal{N}_{2}, \forall l} \frac{h_{2}\left(y_{\text {Gil }} \mid \boldsymbol{\lambda}_{2}\right) \mathrm{e}^{K_{G i l}+b_{l}}}{\exp \left\{\frac{1}{2} H_{2}\left(y_{G i l} \mid \boldsymbol{\lambda}_{2}\right) \mathrm{e}^{K_{G i l}+b_{l}}\right\}} & \prod_{j=1}^{J_{2}} \frac{1}{\lambda_{2 j}} d \boldsymbol{\beta}_{2} d \boldsymbol{\gamma}_{2} d \boldsymbol{\lambda}_{2} \\
& \leq M_{13} \int_{-\infty}^{\infty} \prod_{t=1}^{J_{2}+P_{2}} \frac{\mathrm{e}^{\varphi_{2_{t}}+b_{l}}}{\exp \left\{\frac{1}{2}\left(y_{G i l_{t}}-s_{2 j_{i_{t}}-1}\right) \mathrm{e}^{\varphi_{2_{t}}+b_{l}}\right\}} d \varphi_{2_{t}} \\
& =M_{13} \prod_{l=1}^{J_{2}+P_{2}} \int_{-\infty}^{\infty} \frac{\mathrm{e}^{\varphi_{2_{t}}+b_{l}}}{\exp \left\{\frac{1}{2}\left(y_{G i l}-s_{2 j_{i_{t}}-1}\right) \mathrm{e}^{\varphi_{2_{t}}+b_{l}}\right\}} d \varphi_{2_{t}} .
\end{aligned}
$$

Seja $u=\frac{1}{2}\left(y_{G i l_{t}}-s_{2 j_{i_{l}}-1}\right) \mathrm{e}^{\varphi_{2_{t}}+b_{l}}$, de modo que $d u=\frac{1}{2}\left(y_{G i l_{t}}-s_{2 j_{i_{t}}-1}\right) \mathrm{e}^{\varphi_{2_{t}}+b_{l}} d \varphi_{2_{t}}$. Então,

$$
\int_{-\infty}^{\infty} \frac{\mathrm{e}^{\varphi_{2_{t}}+b_{l}}}{\exp \left\{\mathrm{e}^{\varphi_{2}+b_{l}}\left(y_{G i l_{t}}-s_{2 j_{i_{t}}-1}\right)\right\}} d \varphi_{2 t}=\frac{2}{\left(y_{G i l_{t}}-s_{2 j_{i_{t}}-1}\right)} \int_{0}^{\infty} \mathrm{e}^{-u} d u=\frac{2}{\left(y_{G i l_{t}}-s_{2 j_{i_{t}}-1}\right)} .
$$

Assim,

$$
\int \prod_{i \in \mathfrak{N}\left(\begin{array}{l}
2 \\
,
\end{array} l\right.} \frac{h_{2}\left(y_{G i l} \mid \boldsymbol{\lambda}_{2}\right) \mathrm{e}^{K_{G i l}+b_{l}}}{\exp \left\{\frac{1}{2} H_{2}\left(y_{G i l} \mid \boldsymbol{\lambda}_{2}\right) \mathrm{e}^{K_{G i l}+b_{l}}\right\}} \prod_{j=1}^{J_{2}} \frac{1}{\lambda_{2 j}} d \boldsymbol{\beta}_{2} d \boldsymbol{\gamma}_{2} d \boldsymbol{\lambda}_{2} \leq M_{13} \prod_{t=1}^{J_{2}+P_{2}} \frac{2}{\left(y_{G i l_{t}}-s_{2 j_{i_{t}}-1}\right)}<\infty .
$$


De forma analoga é a resolução de (B.9). Pela condição (C4) em que $\boldsymbol{X}_{1}$ é de posto completo $J_{1}+p_{1}$, existem $J_{1}+p_{1}$ distintos indices $i_{1}, \cdots, i_{J_{1}+p_{1}} \in \mathcal{N}_{2} \cup \mathcal{N}_{3}$ tais que a matriz $\boldsymbol{X}_{\mathbf{1}}^{*}$ de ordem $\left(J_{1}+p_{1}\right) \times\left(J_{1}+p_{1}\right)$ com linhas $\left(\cdots, \delta_{1 j_{i}}, \cdots, A_{i l_{t}}, \boldsymbol{x}_{i l_{t}}^{\top}\right)$ para $t=1, \cdots, J_{1}+p_{1}$, é de posto completo, e obtém-se

$$
\int \prod_{i \in \mathfrak{N}_{2} \cup \mathfrak{N}_{3}, \forall l} \frac{h_{1}\left(y_{E i l} \mid \boldsymbol{\lambda}_{1}\right) \mathrm{e}^{K_{E i l}+b_{l}}}{\exp \left\{\frac{1}{2} H_{1}\left(y_{E i l} \mid \boldsymbol{\lambda}_{1}\right) \mathrm{e}^{K_{E i l}+b_{l}}\right\}} \leq M_{23} \prod_{t=1}^{J_{1}+P_{1}} \frac{2}{\left(y_{E i l_{t}}-s_{1 j_{i_{t}}-1}\right)}<\infty .
$$

Finalmente, pela condição (C4), a distribuição a priori para $\theta$ tem primiero momento finito e portanto (B.10) é finita.

Conclui-se então que sob as condições (C1) a (C5) obtém (3.24), ou seja, assumindo uma distribuição a priori impropia, $\pi^{*}(\boldsymbol{\xi}, B)$, a distribuição a posteriori $\pi^{*}(\boldsymbol{\xi}, B \mid$ $\left.D_{o b s}\right)=\mathrm{L}\left(\boldsymbol{\xi}, B \mid D_{o b s}\right) \pi^{*}(\boldsymbol{\xi}, B)$ é propria. 
Apêndice

\section{Tabelas e figuras das simulações}

Como comentado no Capítulo 4, neste apêndice são apresentadas as tabelas e as figuras resumindo os resultados obtidos no estudo de simulação. 
Tabela C.1: Resumo de 200 replicas com $\theta=1$, censura: $10 \%$ e $\left(J_{0}, J_{1}, J_{2}\right)=(3,3,3)$ segundo a cópula de Clayton. Modelo misto. (Valor: Verdadeiro valor do parâmetro, Est: média das médias a posteriori, SD: média dos desvios padrão a posteriori, RMSE: média das raízes quadradas do erro quadrático médio, CP: probabilidade de cobertura do HPD de 95\%)

\begin{tabular}{|c|c|c|c|c|c|c|c|c|c|}
\hline \multirow[b]{2}{*}{ Parâmetro } & \multirow[b]{2}{*}{ Valor } & \multicolumn{4}{|c|}{$N=500$} & \multicolumn{4}{|c|}{$N=1000$} \\
\hline & & Est & $\mathrm{SD}$ & RMSE & $\mathrm{CP}$ & Est & $\mathrm{SD}$ & RMSE & $\mathrm{CP}$ \\
\hline \multicolumn{10}{|c|}{ Função de risco } \\
\hline$\lambda_{0}$ & 1 & 1,118 & 0,423 & 0,575 & 0,910 & 1,032 & 0,347 & 0,471 & 0,910 \\
\hline$\lambda_{0}$ & 1 & 1,195 & 0,462 & 0,636 & 0,930 & 1,055 & 0,361 & 0,495 & 0,890 \\
\hline$\lambda_{0}$ & 1 & 1,190 & 0,485 & 0,632 & 0,955 & 1,068 & 0,372 & 0,506 & 0,900 \\
\hline$\lambda_{1}$ & 5 & 5,364 & 1,757 & 2,380 & 0,910 & 5,169 & 1,596 & 2,228 & 0,915 \\
\hline$\lambda_{1}$ & 5 & 5,555 & 1,800 & 2,449 & 0,910 & 5,275 & 1,616 & 2,232 & 0,920 \\
\hline$\lambda_{1}$ & 5 & 5,703 & 1,876 & 2,570 & 0,925 & 5,339 & 1,647 & 2,280 & 0,935 \\
\hline$\lambda_{2}$ & 1 & 1,088 & 0,367 & 0,491 & 0,915 & 1,049 & 0,329 & 0,453 & 0,920 \\
\hline$\lambda_{2}$ & 1 & 1,106 & 0,375 & 0,496 & 0,935 & 1,064 & 0,334 & 0,460 & 0,915 \\
\hline$\lambda_{2}$ & 1 & 1,140 & 0,394 & 0,524 & 0,940 & 1,056 & 0,335 & 0,459 & 0,915 \\
\hline \multicolumn{10}{|l|}{ Modelo $T_{D}$} \\
\hline$\beta_{0}$ & -1 & $-1,026$ & 0,200 & 0,271 & 0,945 & $-1,000$ & 0,140 & 0,193 & 0,930 \\
\hline$\gamma_{01}$ & 1 & 1,022 & 0,110 & 0,156 & 0,910 & 1,012 & 0,077 & 0,101 & 0,950 \\
\hline$\gamma_{02}$ & 0,2 & 0,199 & 0,179 & 0,249 & 0,940 & 0,223 & 0,125 & 0,173 & 0,945 \\
\hline \multicolumn{10}{|l|}{ Modelo $T_{E}$} \\
\hline$\beta_{1}$ & $-0,5$ & $-0,514$ & 0,111 & 0,153 & 0,930 & $-0,509$ & 0,078 & 0,105 & 0,955 \\
\hline$\gamma_{11}$ & 1 & 1,011 & 0,068 & 0,091 & 0,955 & 1,006 & 0,047 & 0,065 & 0,955 \\
\hline$\gamma_{12}$ & 0 & $-0,003$ & 0,108 & 0,150 & 0,930 & 0,001 & 0,075 & 0,101 & 0,945 \\
\hline \multicolumn{10}{|l|}{ Modelo $T_{G}$} \\
\hline$\beta_{21}$ & $-0,3$ & $-0,309$ & 0,128 & 0,175 & 0,945 & $-0,300$ & 0,089 & 0,125 & 0,945 \\
\hline$\beta_{22}$ & $-0,5$ & $-0,503$ & 0,121 & 0,166 & 0,955 & $-0,505$ & 0,085 & 0,120 & 0,940 \\
\hline$\gamma_{21}$ & $-0,5$ & $-0,511$ & 0,064 & 0,087 & 0,940 & $-0,506$ & 0,045 & 0,062 & 0,955 \\
\hline$\gamma_{22}$ & 0,5 & 0,506 & 0,115 & 0,153 & 0,955 & 0,500 & 0,081 & 0,109 & 0,960 \\
\hline$\gamma_{23}$ & $-0,4$ & $-0,409$ & 0,083 & 0,116 & 0,915 & $-0,402$ & 0,058 & 0,082 & 0,930 \\
\hline \multicolumn{10}{|l|}{ Modelo logito } \\
\hline $\begin{array}{l}\alpha_{0} \\
\alpha_{1}\end{array}$ & $-1,8$ & $-1,816$ & 0,237 & 0,320 & 0,945 & $-1,806$ & 0,166 & 0,223 & 0,965 \\
\hline$\alpha_{2}$ & 1 & 1,010 & 0,133 & 0,178 & 0,970 & 1,000 & 0,093 & 0,127 & 0,945 \\
\hline$\alpha_{3}$ & 0,1 & 0,111 & 0,225 & 0,299 & 0,955 & 0,103 & 0,158 & 0,218 & 0,940 \\
\hline \multicolumn{10}{|c|}{ Parâmetro cópula } \\
\hline & 1 & 0,986 & 0,148 & 0,198 & 0,955 & 0,995 & 0,104 & 0,138 & 0,970 \\
\hline \multicolumn{10}{|l|}{ Variância EA } \\
\hline$B$ & 1 & 1,027 & 0,439 & 0,538 & 0,985 & 1,031 & 0,436 & 0,541 & 0,975 \\
\hline
\end{tabular}


Tabela C.2: Resumo de 200 replicas com $\theta=1$, censura: $45 \%$ e $\left(J_{0}, J_{1}, J_{2}\right)=(3,3,3)$ segundo a cópula de Clayton. Modelo misto. (Valor: Verdadeiro valor do parâmetro, Est: média das médias a posteriori, SD: média dos desvios padrão a posteriori, RMSE: média das raízes quadradas do erro quadrático médio, CP: probabilidade de cobertura do HPD de 95\%)

\begin{tabular}{|c|c|c|c|c|c|c|c|c|c|}
\hline \multirow[b]{2}{*}{ Parâmetro } & \multirow[b]{2}{*}{ Valor } & \multicolumn{4}{|c|}{$N=500$} & \multicolumn{4}{|c|}{$N=1000$} \\
\hline & & Est & $\mathrm{SD}$ & RMSE & $\mathrm{CP}$ & Est & $\mathrm{SD}$ & RMSE & $\mathrm{CP}$ \\
\hline \multicolumn{10}{|c|}{ Função de risco } \\
\hline$\lambda_{0}$ & 1 & 1,122 & 0,482 & 0,633 & 0,940 & 0,122 & 0,413 & 0,549 & 0,940 \\
\hline$\lambda_{0}$ & 1 & 1,254 & 0,554 & 0,732 & 0,935 & 1,181 & 0,439 & 0,583 & 0,945 \\
\hline$\lambda_{0}$ & 1 & 0,637 & 0,294 & 0,539 & 0,745 & 0,584 & 0,224 & 0,504 & 0,580 \\
\hline$\lambda_{1}$ & 5 & 4,909 & 1,698 & 2,197 & 0,950 & 5,124 & 1,641 & 2,175 & 0,935 \\
\hline$\lambda_{1}$ & 5 & 5,212 & 1,785 & 2,260 & 0,970 & 5,373 & 1,712 & 2,294 & 0,925 \\
\hline$\lambda_{1}$ & 5 & 5,880 & 2,045 & 2,686 & 0,955 & 5,913 & 1,898 & 2,611 & 0,910 \\
\hline$\lambda_{2}$ & 1 & 1,038 & 0,387 & 0,497 & 0,945 & 1,080 & 0,361 & 0,484 & 0,925 \\
\hline$\lambda_{2}$ & 1 & 1,072 & 0,400 & 0,525 & 0,935 & 1,091 & 0,365 & 0,493 & 0,935 \\
\hline$\lambda_{2}$ & 1 & 0,675 & 0,257 & 0,468 & 0,720 & 0,664 & 0,225 & 0,446 & 0,670 \\
\hline \multicolumn{10}{|l|}{ Modelo $T_{D}$} \\
\hline$\beta_{0}$ & -1 & $-1,171$ & 0,247 & 0,372 & 0,900 & $-1,126$ & 0,171 & 0,261 & 0,875 \\
\hline$\gamma_{01}$ & 1 & 1,144 & 0,137 & 0,234 & 0,785 & 1,137 & 0,095 & 0,180 & 0,690 \\
\hline$\gamma_{02}$ & 0,2 & 0,242 & 0,227 & 0,318 & 0,925 & 0,232 & 0,158 & 0,215 & 0,945 \\
\hline \multicolumn{10}{|l|}{ Modelo $T_{E}$} \\
\hline$\beta_{1}$ & $-0,5$ & $-0,517$ & 0,119 & 0,173 & 0,905 & $-0,511$ & 0,082 & 0,113 & 0,950 \\
\hline$\gamma_{11}$ & 1 & 1,045 & 0,074 & 0,107 & 0,905 & 1,036 & 0,051 & 0,076 & 0,885 \\
\hline \multicolumn{10}{|l|}{ Modelo $T_{G}$} \\
\hline$\beta_{21}$ & $-0,3$ & $-0,350$ & 0,161 & 0,237 & 0,890 & $-0,335$ & 0,111 & 0,152 & 0,925 \\
\hline$\beta_{22}$ & $-0,5$ & $-0,552$ & 0,157 & 0,221 & 0,910 & $-0,541$ & 0,108 & 0,153 & 0,920 \\
\hline$\gamma_{21}$ & $-0,5$ & $-0,504$ & 0,081 & 0,107 & 0,975 & $-0,494$ & 0,055 & 0,078 & 0,920 \\
\hline$\gamma_{22}$ & 0,5 & 0,558 & 0,146 & 0,202 & 0,925 & 0,546 & 0,101 & 0,144 & 0,920 \\
\hline \multicolumn{2}{|c|}{ Modelo logito } & $-0,439$ & 0,110 & 0,153 & 0,920 & $-0,437$ & 0,076 & 0,109 & 0,900 \\
\hline$\alpha_{0}$ & 1,6 & 1,665 & 0,239 & 0,325 & 0,950 & 1,654 & 0,166 & 0,230 & 0,950 \\
\hline$\alpha_{1}$ & $-1,8$ & $-1,859$ & 0,247 & 0,346 & 0,930 & $-1,834$ & 0,172 & 0,241 & 0,930 \\
\hline$\alpha_{2}$ & 1 & 1,073 & 0,141 & 0,202 & 0,915 & 1,041 & 0,098 & 0,139 & 0,940 \\
\hline$\alpha_{3}$ & 0,1 & 0,112 & 0,235 & 0,317 & 0,945 & 0,090 & 0,164 & 0,219 & 0,945 \\
\hline \multicolumn{10}{|c|}{ Parâmetro cópula } \\
\hline$\theta$ & 1 & 1,087 & 0,182 & 0,252 & 0,940 & 1,131 & 0,129 & 0,216 & 0,850 \\
\hline Variância & & & & & & & & & \\
\hline$B$ & 1 & 1,119 & 0,485 & 0,590 & 0,995 & 1,071 & 0,454 & 0,561 & 0,985 \\
\hline
\end{tabular}


Tabela C.3: Resumo de 200 replicas com $\theta=1$, censura: $70 \%$ e $\left(J_{0}, J_{1}, J_{2}\right)=(3,3,3)$ segundo a cópula de Clayton. Modelo misto. (Valor: Verdadeiro valor do parâmetro, Est: média das médias a posteriori, SD: média dos desvios padrão a posteriori, RMSE: média das raízes quadradas do erro quadrático médio, CP: probabilidade de cobertura do HPD de 95\%)

\begin{tabular}{|c|c|c|c|c|c|c|c|c|c|}
\hline \multirow[b]{2}{*}{ Parâmetro } & \multirow[b]{2}{*}{ Valor } & \multicolumn{4}{|c|}{$N=500$} & \multicolumn{4}{|c|}{$N=1000$} \\
\hline & & Est & $\mathrm{SD}$ & RMSE & $\mathrm{CP}$ & Est & $\mathrm{SD}$ & RMSE & $\mathrm{CP}$ \\
\hline \multicolumn{10}{|c|}{ Função de risco } \\
\hline$\lambda_{0}$ & 1 & 1,175 & 0,599 & 0,780 & 0,945 & 1,148 & 0,481 & 0,640 & 0,930 \\
\hline$\lambda_{0}$ & 1 & 1,277 & 0,664 & 0,856 & 0,970 & 1,183 & 0,501 & 0,670 & 0,950 \\
\hline$\lambda_{0}$ & 1 & 0,312 & 0,169 & 0,719 & 0,190 & 0,291 & 0,128 & 0,724 & 0,065 \\
\hline$\lambda_{1}$ & 5 & 4,727 & 1,684 & 2,275 & 0,920 & 4,823 & 1,611 & 2,169 & 0,925 \\
\hline$\lambda_{1}$ & 5 & 5,185 & 1,829 & 2,444 & 0,920 & 5,281 & 1,761 & 2,369 & 0,905 \\
\hline$\lambda_{1}$ & 5 & 5,634 & 2,031 & 2,704 & 0,950 & 5,735 & 1,935 & 2,667 & 0,935 \\
\hline$\lambda_{2}$ & 1 & 0,990 & 0,424 & 0,572 & 0,925 & 1,019 & 0,380 & 0,510 & 0,925 \\
\hline$\lambda_{2}$ & 1 & 0,986 & 0,424 & 0,569 & 0,930 & 0,974 & 0,365 & 0,488 & 0,915 \\
\hline$\lambda_{2}$ & 1 & 0,311 & 0,136 & 0,707 & 0,070 & 0,316 & 0,120 & 0,698 & 0,055 \\
\hline \multicolumn{10}{|l|}{ Modelo $T_{D}$} \\
\hline$\beta_{0}$ & -1 & $-1,255$ & 0,331 & 0,496 & 0,890 & $-1,228$ & 0,225 & 0,374 & 0,810 \\
\hline$\gamma_{01}$ & 1 & 1,232 & 0,182 & 0,332 & 0,715 & 1,214 & 0,124 & 0,256 & 0,595 \\
\hline$\gamma_{02}$ & 0,2 & 0,251 & 0,311 & 0,409 & 0,950 & 0,259 & 0,213 & 0,298 & 0,900 \\
\hline \multicolumn{10}{|l|}{ Modelo $T_{E}$} \\
\hline$\beta_{1}$ & $-0,5$ & $-0,524$ & 0,130 & 0,184 & 0,930 & $-0,524$ & 0,089 & 0,127 & 0,920 \\
\hline$\gamma_{11}$ & 1 & 1,057 & 0,079 & 0,119 & 0,910 & 1,057 & 0,055 & 0,092 & 0,795 \\
\hline$\gamma_{12}$ & 0 & 0,006 & 0,125 & 0,166 & 0,965 & $-0,015$ & 0,085 & 0,120 & 0,935 \\
\hline \multicolumn{10}{|l|}{ Modelo $T_{G}$} \\
\hline$\beta_{21}$ & $-0,3$ & $-0,398$ & 0,227 & 0,317 & 0,925 & $-0,411$ & 0,155 & 0,235 & 0,880 \\
\hline$\beta_{22}$ & $-0,5$ & $-0,589$ & 0,226 & 0,309 & 0,950 & $-0,586$ & 0,156 & 0,222 & 0,940 \\
\hline$\gamma_{21}$ & $-0,5$ & $-0,468$ & 0,110 & 0,148 & 0,950 & $-0,456$ & 0,076 & 0,112 & 0,905 \\
\hline$\gamma_{22}$ & 0,5 & 0,613 & 0,208 & 0,294 & 0,910 & 0,583 & 0,143 & 0,211 & 0,875 \\
\hline$\gamma_{23}$ & $-0,4$ & $-0,482$ & 0,162 & 0,228 & 0,930 & $-0,465$ & 0,112 & 0,162 & 0,920 \\
\hline \multicolumn{10}{|l|}{ Modelo logito } \\
\hline $\begin{array}{l}\alpha_{0} \\
\alpha_{1}\end{array}$ & $-1,8$ & $-1,948$ & 0,268 & 0,385 & 0,915 & $-1,917$ & 0,185 & 0,273 & 0,890 \\
\hline$\alpha_{2}$ & 1 & 1,109 & 0,151 & 0,224 & 0,910 & 1,082 & 0,104 & 0,157 & 0,885 \\
\hline$\alpha_{3}$ & 0,1 & 0,058 & 0,250 & 0,345 & 0,930 & 0,091 & 0,173 & 0,241 & 0,915 \\
\hline \multicolumn{10}{|c|}{ Parâmetro cópula } \\
\hline$\theta$ & 1 & 1,376 & 0,279 & 0,503 & 0,720 & 1,394 & 0,192 & 0,447 & 0,425 \\
\hline Variância EA & & & & & & & & & \\
\hline$B$ & 1 & 1,158 & 0,500 & 0,611 & 0,975 & 1,160 & 0,498 & 0,627 & 0,950 \\
\hline
\end{tabular}


Tabela C.4: Resumo de 200 replicas com $\theta=2$, censura: $10 \%$ e $\left(J_{0}, J_{1}, J_{2}\right)=(3,3,3)$ segundo a cópula de Clayton. Modelo misto. (Valor: Verdadeiro valor do parâmetro, Est: média das médias a posteriori, SD: média dos desvios padrão a posteriori, RMSE: média das raízes quadradas do erro quadrático médio, CP: probabilidade de cobertura do HPD de 95\%)

\begin{tabular}{|c|c|c|c|c|c|c|c|c|c|}
\hline \multirow[b]{2}{*}{ Parâmetro } & \multirow[b]{2}{*}{ Valor } & \multicolumn{4}{|c|}{$N=500$} & \multicolumn{4}{|c|}{$N=1000$} \\
\hline & & Est & $\mathrm{SD}$ & RMSE & $\mathrm{CP}$ & Est & $\mathrm{SD}$ & RMSE & $\mathrm{CP}$ \\
\hline \multicolumn{10}{|c|}{ Função de risco } \\
\hline$\lambda_{0}$ & 1 & 1,093 & 0,413 & 0,555 & 0,935 & 1,106 & 0,372 & 0,502 & 0,935 \\
\hline$\lambda_{0}$ & 1 & 1,150 & 0,446 & 0,595 & 0,945 & 1,119 & 0,382 & 0,508 & 0,945 \\
\hline$\lambda_{0}$ & 1 & 1,146 & 0,462 & 0,616 & 0,960 & 1,123 & 0,392 & 0,520 & 0,945 \\
\hline$\lambda_{1}$ & 5 & 5,228 & 1,676 & 2,241 & 0,935 & 5,423 & 1,663 & 2,234 & 0,950 \\
\hline$\lambda_{1}$ & 5 & 5,363 & 1,693 & 2,250 & 0,945 & 5,451 & 1,652 & 2,232 & 0,950 \\
\hline$\lambda_{1}$ & 5 & 5,482 & 1,756 & 2,337 & 0,950 & 5,510 & 1,682 & 2,238 & 0,945 \\
\hline$\lambda_{2}$ & 1 & 1,058 & 0,343 & 0,458 & 0,940 & 1,087 & 0,334 & 0,449 & 0,950 \\
\hline$\lambda_{2}$ & 1 & 1,074 & 0,349 & 0,465 & 0,950 & 1,084 & 0,334 & 0,447 & 0,945 \\
\hline$\lambda_{2}$ & 1 & 1,108 & 0,364 & 0,490 & 0,955 & 1,098 & 0,340 & 0,456 & 0,945 \\
\hline \multicolumn{10}{|l|}{ Modelo $T_{D}$} \\
\hline$\beta_{0}$ & -1 & $-1,027$ & 0,201 & 0,266 & 0,980 & $-1,019$ & 0,139 & 0,188 & 0,945 \\
\hline$\gamma_{01}$ & 1 & 1,026 & 0,110 & 0,150 & 0,950 & 1,007 & 0,076 & 0,102 & 0,970 \\
\hline$\gamma_{02}$ & 0,2 & 0,221 & 0,180 & 0,263 & 0,895 & 0,207 & 0,124 & 0,171 & 0,940 \\
\hline \multicolumn{10}{|l|}{ Modelo $T_{E}$} \\
\hline$\beta_{1}$ & $-0,5$ & $-0,501$ & 0,103 & 0,143 & 0,930 & $-0,502$ & 0,071 & 0,097 & 0,920 \\
\hline$\gamma_{11}$ & 1 & 1,010 & 0,063 & 0,086 & 0,930 & 1,000 & 0,043 & 0,058 & 0,940 \\
\hline \multicolumn{10}{|l|}{ Modelo $T_{G}$} \\
\hline$\beta_{21}$ & $-0,3$ & $-0,298$ & 0,113 & 0,154 & 0,945 & $-0,295$ & 0,078 & 0,109 & 0,925 \\
\hline$\beta_{22}$ & $-0,5$ & $-0,505$ & 0,088 & 0,120 & 0,955 & $-0,501$ & 0,060 & 0,078 & 0,975 \\
\hline$\gamma_{21}$ & $-0,5$ & $-0,502$ & 0,059 & 0,081 & 0,935 & $-0,507$ & 0,041 & 0,054 & 0,970 \\
\hline$\gamma_{22}$ & 0,5 & 0,519 & 0,105 & 0,146 & 0,940 & 0,504 & 0,072 & 0,093 & 0,995 \\
\hline \multicolumn{9}{|c|}{ Modelo logito } & 0,950 \\
\hline$\alpha_{0}$ & 1,6 & 1,633 & 0,229 & 0,318 & 0,930 & 1,626 & 0,161 & 0,219 & 0,955 \\
\hline$\alpha_{1}$ & $-1,8$ & $-1,827$ & 0,237 & 0,327 & 0,940 & $-1,838$ & 0,166 & 0,229 & 0,955 \\
\hline$\alpha_{2}$ & 1 & 1,017 & 0,133 & 0,186 & 0,955 & 1,017 & 0,093 & 0,124 & 0,945 \\
\hline$\alpha_{3}$ & 0,1 & 0,095 & 0,226 & 0,307 & 0,935 & 0,099 & 0,159 & 0,220 & 0,945 \\
\hline \multicolumn{10}{|c|}{ Parâmetro cópula } \\
\hline$\theta$. & 2 & 1,989 & 0,227 & 0,311 & 0,950 & 2,004 & 0,158 & 0,214 & 0,970 \\
\hline $\begin{array}{c}\text { Variância } \\
R\end{array}$ & & & & & & & & & \\
\hline$B$ & 1 & 0,985 & 0,421 & 0,517 & 1,000 & 1,024 & 0,436 & 0,538 & 0,980 \\
\hline
\end{tabular}


Tabela C.5: Resumo de 200 replicas com $\theta=2$, censura: $45 \%$ e $\left(J_{0}, J_{1}, J_{2}\right)=(3,3,3)$ segundo a cópula de Clayton. Modelo misto. (Valor: Verdadeiro valor do parâmetro, Est: média das médias a posteriori, SD: média dos desvios padrão a posteriori, RMSE: média das raízes quadradas do erro quadrático médio, CP: probabilidade de cobertura do HPD de 95\%)

\begin{tabular}{|c|c|c|c|c|c|c|c|c|c|}
\hline \multirow[b]{2}{*}{ Parâmetro } & \multirow[b]{2}{*}{ Valor } & \multicolumn{4}{|c|}{$N=500$} & \multicolumn{4}{|c|}{$N=1000$} \\
\hline & & Est & SD & RMSE & $\mathrm{CP}$ & Est & SD & RMSE & $\mathrm{CP}$ \\
\hline \multicolumn{10}{|l|}{ Fun de risco } \\
\hline$\lambda_{0}$ & 1 & 1,129 & 0,484 & 0,625 & 0,930 & 1,088 & 0,400 & 0,523 & 0,965 \\
\hline$\lambda_{0}$ & 1 & 1,179 & 0,516 & 0,682 & 0,935 & 1,145 & 0,426 & 0,562 & 0,955 \\
\hline$\lambda_{0}$ & 1 & 0,594 & 0,272 & 0,539 & 0,675 & 0,578 & 0,222 & 0,509 & 0,585 \\
\hline$\lambda_{1}$ & 5 & 4,863 & 1,656 & 2,168 & 0,950 & 4,815 & 1,547 & 2,015 & 0,935 \\
\hline$\lambda_{1}$ & 5 & 5,254 & 1,776 & 2,302 & 0,955 & 5,033 & 1,607 & 2,062 & 0,940 \\
\hline$\lambda_{1}$ & 5 & 5,900 & 2,028 & 2,699 & 0,950 & 5,683 & 1,827 & 2,444 & 0,940 \\
\hline$\lambda_{2}$ & 1 & 1,019 & 0,365 & 0,465 & 0,955 & 1,014 & 0,335 & 0,431 & 0,960 \\
\hline$\lambda_{2}$ & 1 & 1,088 & 0,390 & 0,513 & 0,965 & 1,078 & 0,355 & 0,462 & 0,965 \\
\hline$\lambda_{2}$ & 1 & 0,732 & 0,268 & 0,444 & 0,750 & 0,705 & 0,235 & 0,422 & 0,750 \\
\hline \multicolumn{10}{|l|}{ Modelo $T_{D}$} \\
\hline$\beta_{0}$ & -1 & $-1,136$ & 0,248 & 0,363 & 0,915 & $-1,129$ & 0,171 & 0,270 & 0,855 \\
\hline$\gamma_{01}$ & 1 & 1,136 & 0,136 & 0,231 & 0,810 & 1,128 & 0,095 & 0,175 & 0,705 \\
\hline \multicolumn{5}{|l|}{ Modelo $T_{E}$} & 0,940 & 0,228 & 0,157 & 0,216 & 0,935 \\
\hline$\beta_{1}$ & $-0,5$ & $-0,523$ & 0,112 & 0,154 & 0,945 & $-0,524$ & 0,078 & 0,112 & 0,925 \\
\hline$\gamma_{11}$ & 1 & 1,050 & 0,070 & 0,106 & 0,900 & 1,039 & 0,048 & 0,076 & 0,840 \\
\hline$\gamma_{12}$ & 0 & $-0,002$ & 0,108 & 0,153 & 0,950 & $-0,002$ & 0,075 & 0,100 & 0,955 \\
\hline \multicolumn{10}{|l|}{ Modelo $T_{G}$} \\
\hline$\beta_{21}$ & $-0,3$ & $-0,326$ & 0,142 & 0,190 & 0,940 & $-0,347$ & 0,098 & 0,145 & 0,885 \\
\hline$\beta_{22}$ & $-0,5$ & $-0,543$ & 0,123 & 0,169 & 0,940 & $-0,530$ & 0,085 & 0,116 & 0,935 \\
\hline$\gamma_{21}$ & $-0,5$ & $-0,499$ & 0,073 & 0,100 & 0,945 & $-0,500$ & 0,051 & 0,070 & 0,910 \\
\hline$\gamma_{22}$ & 0,5 & 0,551 & 0,132 & 0,186 & 0,945 & 0,535 & 0,091 & 0,125 & 0,945 \\
\hline \multicolumn{9}{|l|}{ Modelo logito } & 0,890 \\
\hline$\alpha_{0}$ & 1,6 & 1,690 & 0,237 & 0,328 & 0,920 & 1,666 & 0,166 & 0,234 & 0,940 \\
\hline$\alpha_{1}$ & $-1,8$ & $-1,904$ & 0,249 & 0,352 & 0,920 & $-1,843$ & 0,172 & 0,238 & 0,940 \\
\hline$\alpha_{2}$ & 1 & 1,064 & 0,140 & 0,199 & 0,935 & 1,044 & 0,098 & 0,138 & 0,935 \\
\hline$\alpha_{3}$ & 0,1 & 0,114 & 0,235 & 0,316 & 0,955 & 0,086 & 0,164 & 0,219 & 0,965 \\
\hline \multicolumn{10}{|c|}{ Parâmetro cópula } \\
\hline$\theta$ & 2 & 2,120 & 0,276 & 0,389 & 0,925 & 2,090 & 0,189 & 0,300 & 0,875 \\
\hline Variância EA & & & & & & & & & \\
\hline$B$ & 1 & 1,118 & 0,478 & 0,599 & 0,975 & 1,110 & 0,471 & 0,588 & 0,950 \\
\hline
\end{tabular}


Tabela C.6: Resumo de 200 replicas com $\theta=2$, censura: $70 \%$ e $\left(J_{0}, J_{1}, J_{2}\right)=(3,3,3)$ segundo a cópula de Clayton. Modelo misto. (Valor: Verdadeiro valor do parâmetro, Est: média das médias a posteriori, SD: média dos desvios padrão a posteriori, RMSE: média das raízes quadradas do erro quadrático médio, CP: probabilidade de cobertura do HPD de 95\%)

\begin{tabular}{|c|c|c|c|c|c|c|c|c|c|}
\hline \multirow[b]{2}{*}{ Parâmetro } & \multirow[b]{2}{*}{ Valor } & \multicolumn{4}{|c|}{$N=500$} & \multicolumn{4}{|c|}{$N=1000$} \\
\hline & & Est & SD & RMSE & $\mathrm{CP}$ & Est & SD & RMSE & $\mathrm{CP}$ \\
\hline \multicolumn{10}{|l|}{ Fun de risco } \\
\hline$\lambda_{0}$ & 1 & 1,120 & 0,566 & 0,725 & 0,960 & 1,139 & 0,474 & 0,625 & 0,945 \\
\hline$\lambda_{0}$ & 1 & 1,193 & 0,612 & 0,783 & 0,985 & 1,167 & 0,492 & 0,651 & 0,940 \\
\hline$\lambda_{0}$ & 1 & 0,296 & 0,160 & 0,730 & 0,170 & 0,278 & 0,121 & 0,735 & 0,045 \\
\hline$\lambda_{1}$ & 5 & 4,524 & 1,539 & 2,186 & 0,890 & 4,667 & 1,541 & 2,058 & 0,930 \\
\hline$\lambda_{1}$ & 5 & 5,020 & 1,692 & 2,246 & 0,950 & 5,134 & 1,692 & 2,217 & 0,945 \\
\hline$\lambda_{1}$ & 5 & 5,495 & 1,900 & 2,529 & 0,930 & 5,619 & 1,884 & 2,461 & 0,940 \\
\hline$\lambda_{2}$ & 1 & 0,962 & 0,384 & 0,517 & 0,920 & 1,001 & 0,363 & 0,478 & 0,925 \\
\hline$\lambda_{2}$ & 1 & 0,985 & 0,394 & 0,524 & 0,930 & 1,015 & 0,368 & 0,492 & 0,950 \\
\hline$\lambda_{2}$ & 1 & 0,368 & 0,150 & 0,658 & 0,135 & 0,370 & 0,136 & 0,650 & 0,105 \\
\hline \multicolumn{10}{|l|}{ Modelo $T_{D}$} \\
\hline$\beta_{0}$ & -1 & $-1,272$ & 0,333 & 0,520 & 0,86 & $-1,213$ & 0,227 & 0,370 & 0,845 \\
\hline$\gamma_{01}$ & 1 & 1,259 & 0,185 & 0,343 & 0,760 & 1,225 & 0,126 & 0,272 & 0,585 \\
\hline$\gamma_{02}$ & 0,2 & 0,283 & 0,312 & 0,439 & 0,925 & 0,260 & 0,214 & 0,305 & 0,930 \\
\hline \multicolumn{10}{|l|}{ Modelo $T_{E}$} \\
\hline$\beta_{1}$ & $-0,5$ & $-0,534$ & 0,125 & 0,179 & 0,915 & $-0,523$ & 0,086 & 0,118 & 0,955 \\
\hline$\gamma_{11}$ & 1 & 1,070 & 0,077 & 0,123 & 0,86 & 1,064 & 0,053 & 0,093 & 0,775 \\
\hline$\gamma_{12}$ & 0 & $-0,019$ & 0,120 & 0,159 & 0,955 & $-0,007$ & 0,082 & 0,112 & 0,935 \\
\hline \multicolumn{10}{|l|}{ Modelo $T_{G}$} \\
\hline$\beta_{21}$ & $-0,3$ & $-0,392$ & 0,203 & 0,281 & 0,920 & $-0,370$ & 0,139 & 0,197 & 0,915 \\
\hline$\beta_{22}$ & $-0,5$ & $-0,580$ & 0,194 & 0,264 & 0,945 & $-0,560$ & 0,132 & 0,188 & 0,915 \\
\hline$\gamma_{21}$ & $-0,5$ & $-0,469$ & 0,102 & 0,139 & 0,96 & $-0,454$ & 0,071 & 0,104 & 0,895 \\
\hline$\gamma_{22}$ & 0,5 & 0,564 & 0,191 & 0,263 & 0,915 & 0,564 & 0,131 & 0,184 & 0,920 \\
\hline \multicolumn{5}{|l|}{ Modelo logito } & 0,940 & $-0,461$ & 0,096 & 0,139 & 0,910 \\
\hline$\alpha_{0}$ & 1,6 & 1,800 & 0,260 & 0,388 & 0,915 & 1,747 & 0,179 & 0,270 & 0,885 \\
\hline$\alpha_{1}$ & $-1,8$ & $-1,982$ & 0,267 & 0,394 & 0,885 & $-1,899$ & 0,185 & 0,264 & 0,930 \\
\hline$\alpha_{2}$ & 1 & 1,108 & 0,151 & 0,226 & 0,870 & 1,079 & 0,104 & 0,156 & 0,905 \\
\hline$\alpha_{3}$ & 0,1 & 0,107 & 0,251 & 0,335 & 0,955 & 0,092 & 0,173 & 0,237 & 0,950 \\
\hline \multicolumn{10}{|c|}{ Parâmetro cópula } \\
\hline \multirow{2}{*}{\multicolumn{10}{|c|}{ Variância EA }} \\
\hline & & & & & & & & & \\
\hline$B$ & 1 & 1,087 & 0,474 & 0,600 & 0,965 & 1,137 & 0,489 & 0,619 & 0,960 \\
\hline
\end{tabular}


Tabela C.7: Resumo de 200 replicas de tamanho $N=1000$, com $\theta=1$, censura: $10 \%$ e $\left(J_{0}, J_{1}, J_{2}\right)=(3,3,3)$ segundo a cópula de Clayton. Modelo misto. Dados gerados com distribuição Weibull.(Valor: Verdadeiro valor do parâmetro, Est: média das médias a posteriori, SD: média dos desvios padrão a posteriori, RMSE: média das raizes quadradas do erro quadrático médio, CP: probabilidade de cobertura do HPD de 95\%)

\begin{tabular}{cc|cccc}
\hline \hline Parâmetro & Valor & Est & SD & RMSE & CP \\
\hline Função de risco & & & & & \\
$\lambda_{0}$ & & 0,664 & 0,257 & 0,475 & 0,725 \\
$\lambda_{0}$ & & 2,046 & 0,804 & 1,362 & 0,660 \\
$\lambda_{0}$ & & 3,805 & 1,540 & 3,218 & 0,195 \\
$\lambda_{1}$ & & 2,367 & 0,866 & 2,828 & 0,340 \\
$\lambda_{1}$ & & 6,740 & 2,429 & 3,299 & 0,955 \\
$\lambda_{1}$ & & 13,379 & 4,896 & 9,781 & 0,325 \\
$\lambda_{2}$ & & 0,624 & 0,228 & 0,476 & 0,645 \\
$\lambda_{2}$ & & 1,607 & 0,585 & 0,899 & 0,825 \\
$\lambda_{2}$ & & 2,677 & 0,991 & 1,969 & 0,335 \\
Modelo $T_{D}$ & & & & & \\
$\beta_{0}$ & -1 & $-1,548$ & 0,142 & 0,567 & 0,010 \\
$\gamma_{01}$ & 1 & 1,545 & 0,083 & 0,552 & 0,000 \\
$\gamma_{02}$ & 0,2 & 0,314 & 0,125 & 0,191 & 0,890 \\
Modelo $T_{E}$ & & & & & \\
$\beta_{1}$ & $-0,5$ & $-0,712$ & 0,076 & 0,226 & 0,145 \\
$\gamma_{11}$ & 1 & 1,423 & 0,051 & 0,426 & 0,000 \\
$\gamma_{12}$ & 0 & 0,003 & 0,073 & 0,090 & 0,985 \\
Modelo $T_{G}$ & & & & & \\
$\beta_{21}$ & $-0,3$ & $-0,436$ & 0,084 & 0,166 & 0,620 \\
$\beta_{22}$ & $-0,5$ & $-0,736$ & 0,074 & 0,248 & 0,070 \\
$\gamma_{21}$ & $-0,5$ & $-0,728$ & 0,045 & 0,233 & 0,000 \\
$\gamma_{22}$ & 0,5 & 0,733 & 0,078 & 0,247 & 0,110 \\
$\gamma_{23}$ & $-0,4$ & $-0,593$ & 0,051 & 0,200 & 0,025 \\
Modelo logito & & & & & \\
$\alpha_{0}$ & 1,6 & 1,613 & 0,160 & 0,215 & 0,955 \\
$\alpha_{1}$ & $-1,8$ & $-1,801$ & 0,166 & 0,221 & 0,955 \\
$\alpha_{2}$ & 1 & 0,984 & 0,094 & 0,129 & 0,940 \\
$\alpha_{3}$ & 0,1 & 0,114 & 0,157 & 0,228 & 0,935 \\
Parâmetro cópula & & & & & \\
$\theta$ & 1 & 1,493 & 0,153 & 0,518 & 0,060 \\
Variância EA & & & & & \\
$B$ & 1 & 1,653 & 0,701 & 1,003 & 0,790 \\
\hline \hline & & & & & \\
\hline \hline
\end{tabular}


Tabela C.8: Resumo de 200 replicas de tamanho $N=500$, com $\theta=1$, censura: $45 \%$ e $\left(J_{0}, J_{1}, J_{2}\right)=(3,3,3)$ segundo a cópula de Clayton. Modelo misto. Dados gerados com distribuição Weibull.(Valor: Verdadeiro valor do parâmetro, Est: média das médias a posteriori, SD: média dos desvios padrão a posteriori, RMSE: média das raízes quadradas do erro quadrático médio, CP: probabilidade de cobertura do HPD de 95\%)

\begin{tabular}{cc|cccc}
\hline \hline Parâmetro & Valor & Est & SD & RMSE & CP \\
\hline Função de risco & & & & & \\
$\lambda_{0}$ & & 0,486 & 0,239 & 0,595 & 0,490 \\
$\lambda_{0}$ & & 1,675 & 0,834 & 1,149 & 0,920 \\
$\lambda_{0}$ & & 1,888 & 0,992 & 1,388 & 0,915 \\
$\lambda_{1}$ & & 1,931 & 0,800 & 3,196 & 0,130 \\
$\lambda_{1}$ & & 6,771 & 2,713 & 3,506 & 0,950 \\
$\lambda_{1}$ & & 16,180 & 6,612 & 13,063 & 0,255 \\
$\lambda_{2}$ & & 0,481 & 0,205 & 0,574 & 0,395 \\
$\lambda_{2}$ & & 1,348 & 0,567 & 0,744 & 0,940 \\
$\lambda_{2}$ & & 1,744 & 0,749 & 1,100 & 0,870 \\
Modelo $T_{D}$ & & & & & \\
$\beta_{0}$ & -1 & $-1,762$ & 0,257 & 0,809 & 0,115 \\
$\gamma_{01}$ & 1 & 1,783 & 0,154 & 0,799 & 0,000 \\
$\gamma_{02}$ & 0,2 & 0,363 & 0,233 & 0,330 & 0,930 \\
Modelo $T_{E}$ & & & & & \\
$\beta_{1}$ & $-0,5$ & $-0,758$ & 0,118 & 0,289 & 0,395 \\
$\gamma_{11}$ & 1 & 1,527 & 0,078 & 0,533 & 0,000 \\
$\gamma_{12}$ & 0 & 0,007 & 0,113 & 0,145 & 0,965 \\
Modelo $T_{G}$ & & & & & \\
$\beta_{21}$ & $-0,3$ & $-0,499$ & 0,153 & 0,265 & 0,800 \\
$\beta_{22}$ & $-0,5$ & $-0,819$ & 0,142 & 0,354 & 0,345 \\
$\gamma_{21}$ & $-0,5$ & $-0,784$ & 0,084 & 0,297 & 0,095 \\
$\gamma_{22}$ & 0,5 & 0,820 & 0,142 & 0,355 & 0,385 \\
$\gamma_{23}$ & $-0,4$ & $-0,663$ & 0,102 & 0,286 & 0,265 \\
Modelo logito & & & & & \\
$\alpha_{0}$ & 1,6 & 1,693 & 0,237 & 0,327 & 0,925 \\
$\alpha_{1}$ & $-1,8$ & $-1,891$ & 0,247 & 0,341 & 0,935 \\
$\alpha_{2}$ & 1 & 1,039 & 0,140 & 0,189 & 0,955 \\
$\alpha_{3}$ & 0,1 & 0,107 & 0,233 & 0,318 & 0,93 \\
Parâmetro cópula & & & & & \\
$\theta$ & 1 & 1,336 & 0,235 & 0,721 & 0,235 \\
Variância EA & & & & & \\
$B$ & 1 & 2,010 & 0,859 & 1,364 & 0,585 \\
\hline \hline & & & & &
\end{tabular}


Tabela C.9: Resumo de 200 replicas $\operatorname{com} \theta=1$, censura: $10 \%$ e $\left(J_{0}, J_{1}, J_{2}\right)=(3,3,3)$ segundo a cópula de Clayton. Modelo fixo. (Valor: Verdadeiro valor do parâmetro, Est: média das médias a posteriori, SD: média dos desvios padrão a posteriori, RMSE: média das raízes quadradas do erro quadrático médio, CP: probabilidade de cobertura do HPD de 95\%)

\begin{tabular}{|c|c|c|c|c|c|c|c|c|c|}
\hline \multirow{3}{*}{ Parâmetro } & \multirow[b]{2}{*}{ Valor } & \multicolumn{4}{|c|}{$N=500$} & \multicolumn{4}{|c|}{$N=1000$} \\
\hline & & Est & SD & RMSE & $\mathrm{CP}$ & Est & SD & RMSE & $\mathrm{CP}$ \\
\hline & \\
\hline$\lambda_{0}$ & 1 & 1,030 & 0,219 & 0,297 & 0,955 & 1,020 & 0,151 & 0,195 & 0,980 \\
\hline$\lambda_{0}$ & 1 & 1,073 & 0,247 & 0,337 & 0,915 & 1,039 & 0,166 & 0,218 & 0,975 \\
\hline$\lambda_{0}$ & 1 & 1,108 & 0,284 & 0,382 & 0,960 & 1,042 & 0,186 & 0,246 & 0,955 \\
\hline$\lambda_{1}$ & 5 & 4,940 & 0,644 & 0,896 & 0,925 & 4,963 & 0,454 & 0,610 & 0,965 \\
\hline$\lambda_{1}$ & 5 & 5,061 & 0,607 & 0,822 & 0,960 & 4,985 & 0,423 & 0,565 & 0,970 \\
\hline$\lambda_{1}$ & 5 & 5,214 & 0,654 & 0,888 & 0,950 & 5,069 & 0,447 & 0,591 & 0,965 \\
\hline$\lambda_{2}$ & 1 & 1,020 & 0,145 & 0,199 & 0,930 & 0,995 & 0,099 & 0,135 & 0,950 \\
\hline$\lambda_{2}$ & 1 & 1,033 & 0,149 & 0,201 & 0,940 & 1,003 & 0,101 & 0,135 & 0,955 \\
\hline$\lambda_{2}$ & 1 & 1,060 & 0,165 & 0,224 & 0,950 & 1,006 & 0,109 & 0,147 & 0,940 \\
\hline \multicolumn{10}{|l|}{ Modelo $T_{D}$} \\
\hline$\beta_{0}$ & -1 & $-1,019$ & 0,194 & 0,264 & 0,945 & $-1,024$ & 0,135 & 0,180 & 0,985 \\
\hline$\gamma_{01}$ & 1 & 1,022 & 0,112 & 0,151 & 0,955 & 1,010 & 0,078 & 0,106 & 0,960 \\
\hline$\gamma_{02}$ & 0,2 & 0,198 & 0,172 & 0,236 & 0,915 & 0,205 & 0,120 & 0,162 & 0,940 \\
\hline \multicolumn{10}{|l|}{ Modelo $T_{E}$} \\
\hline$\beta_{1}$ & $-0,5$ & $-0,506$ & 0,108 & 0,146 & 0,940 & $-0,505$ & 0,075 & 0,100 & 0,955 \\
\hline$\gamma_{11}$ & 1 & 1,014 & 0,069 & 0,093 & 0,945 & 1,003 & 0,048 & 0,066 & 0,935 \\
\hline$\gamma_{12}$ & 0 & 0,002 & 0,104 & 0,143 & 0,955 & 0,007 & 0,073 & 0,098 & 0,950 \\
\hline \multicolumn{10}{|l|}{ Modelo $T_{G}$} \\
\hline$\beta_{21}$ & $-0,3$ & $-0,317$ & 0,122 & 0,168 & 0,940 & $-0,299$ & 0,085 & 0,114 & 0,950 \\
\hline$\beta_{22}$ & $-0,5$ & $-0,530$ & 0,114 & 0,155 & 0,950 & $-0,502$ & 0,080 & 0,108 & 0,960 \\
\hline$\gamma_{21}$ & $-0,5$ & $-0,511$ & 0,062 & 0,085 & 0,935 & $-0,506$ & 0,043 & 0,062 & 0,925 \\
\hline$\gamma_{22}$ & 0,5 & 0,504 & 0,110 & 0,147 & 0,975 & 0,511 & 0,077 & 0,103 & 0,960 \\
\hline $\begin{array}{c}\gamma_{23} \\
\text { Modelo logit }\end{array}$ & \multicolumn{8}{|c|}{ Modelo logito } & 0,930 \\
\hline$\alpha_{0}$ & 1,6 & 1,630 & 0,226 & 0,308 & 0,950 & 1,595 & 0,159 & 0,216 & 0,945 \\
\hline$\alpha_{1}$ & $-1,8$ & $-1,831$ & 0,234 & 0,328 & 0,945 & $-1,793$ & 0,164 & 0,221 & 0,960 \\
\hline$\alpha_{2}$ & 1 & 1,038 & 0,131 & 0,180 & 0,945 & 1,030 & 0,092 & 0,136 & 0,875 \\
\hline$\alpha_{3}$ & 0,1 & 0,104 & 0,224 & 0,303 & 0,950 & 0,110 & 0,157 & 0,217 & 0,930 \\
\hline \multicolumn{10}{|c|}{ Parâmetro cópula } \\
\hline$\theta$ & 1 & 0,995 & 0,141 & 0,196 & 0,945 & 0,995 & 0,100 & 0,133 & 0,960 \\
\hline
\end{tabular}


Tabela C.10: Resumo de 200 replicas com $\theta=1$, censura: $45 \%$ e $\left(J_{0}, J_{1}, J_{2}\right)=(3,3,3)$ segundo a cópula de Clayton. Modelo fixo. (Valor: Verdadeiro valor do parâmetro, Est: média das médias a posteriori, SD: média dos desvios padrão a posteriori, RMSE: média das raízes quadradas do erro quadrático médio, CP: probabilidade de cobertura do HPD de 95\%)

\begin{tabular}{|c|c|c|c|c|c|c|c|c|c|}
\hline \multirow[b]{2}{*}{ Parâmetro } & \multirow[b]{2}{*}{ Valor } & \multicolumn{4}{|c|}{$\overline{P N=500}$} & \multicolumn{4}{|c|}{$N=1000$} \\
\hline & & Est & SD & RMSE & $\mathrm{CP}$ & Est & SD & RMSE & $\mathrm{CP}$ \\
\hline \multicolumn{10}{|l|}{ Função de risco } \\
\hline$\lambda_{0}$ & 1 & 1,085 & 0,297 & 0,407 & 0,930 & 1,066 & 0,205 & 0,278 & 0,955 \\
\hline$\lambda_{0}$ & 1 & 1,137 & 0,323 & 0,442 & 0,960 & 1,105 & 0,219 & 0,298 & 0,940 \\
\hline$\lambda_{0}$ & 1 & 0,513 & 0,162 & 0,522 & 0,280 & 0,504 & 0,111 & 0,512 & 0,060 \\
\hline$\lambda_{1}$ & 5 & 4,924 & 0,669 & 0,902 & 0,950 & 4,897 & 0,468 & 0,656 & 0,890 \\
\hline$\lambda_{1}$ & 5 & 5,046 & 0,637 & 0,865 & 0,940 & 4,951 & 0,438 & 0,603 & 0,935 \\
\hline$\lambda_{1}$ & 5 & 5,255 & 0,689 & 0,973 & 0,920 & 5,118 & 0,472 & 0,663 & 0,940 \\
\hline$\lambda_{2}$ & 1 & 1,002 & 0,188 & 0,270 & 0,920 & 1,006 & 0,132 & 0,182 & 0,950 \\
\hline$\lambda_{2}$ & 1 & 1,004 & 0,189 & 0,264 & 0,910 & 1,000 & 0,132 & 0,187 & 0,915 \\
\hline$\lambda_{2}$ & 1 & 0,610 & 0,120 & 0,417 & 0,195 & 0,603 & 0,083 & 0,407 & 0,035 \\
\hline \multicolumn{10}{|l|}{ Modelo $T_{D}$} \\
\hline$\beta_{0}$ & -1 & $-1,174$ & 0,247 & 0,365 & 0,905 & $-1,171$ & 0,173 & 0,279 & 0,835 \\
\hline$\gamma_{01}$ & 1 & 1,190 & 0,142 & 0,256 & 0,740 & 1,189 & 0,098 & 0,222 & 0,515 \\
\hline $\begin{array}{c}\gamma_{02} \\
\text { Modelo } T_{F}\end{array}$ & 0,2 & 0,254 & 0,230 & 0,334 & 0,915 & 0,234 & 0,159 & 0,216 & 0,935 \\
\hline$\beta_{1}$ & $-0,5$ & $-0,500$ & 0,116 & 0,158 & 0,950 & $-0,496$ & 0,081 & 0,109 & 0,945 \\
\hline$\gamma_{11}$ & 1 & 1,022 & 0,075 & 0,105 & 0,935 & 1,006 & 0,052 & 0,072 & 0,910 \\
\hline $\begin{array}{c}\gamma_{12} \\
\text { Modelo } T_{G}\end{array}$ & 0 & $-0,012$ & 0,111 & 0,148 & 0,955 & 0,008 & 0,078 & 0,109 & 0,940 \\
\hline$\beta_{21}$ & $-0,3$ & $-0,360$ & 0,159 & 0,224 & 0,920 & $-0,351$ & 0,111 & 0,160 & 0,945 \\
\hline$\beta_{22}$ & $-0,5$ & $-0,574$ & 0,157 & 0,226 & 0,910 & $-0,588$ & 0,109 & 0,169 & 0,870 \\
\hline$\gamma_{21}$ & $-0,5$ & $-0,529$ & 0,080 & 0,115 & 0,925 & $-0,528$ & 0,056 & 0,079 & 0,920 \\
\hline$\gamma_{22}$ & 0,5 & 0,605 & 0,147 & 0,227 & 0,870 & 0,589 & 0,102 & 0,164 & 0,825 \\
\hline \multicolumn{10}{|l|}{ Modelo logito } \\
\hline$\alpha_{0}$ & 1,6 & 1,657 & 0,234 & 0,320 & 0,940 & 1,651 & 0,165 & 0,238 & 0,915 \\
\hline$\alpha_{1}$ & $-1,8$ & $-1,876$ & 0,243 & 0,329 & 0,945 & $-1,870$ & 0,172 & 0,244 & 0,935 \\
\hline$\alpha_{2}$ & 1 & 1,060 & 0,138 & 0,188 & 0,930 & 1,062 & 0,097 & 0,137 & 0,910 \\
\hline$\alpha_{3}$ & 0,1 & 0,100 & 0,232 & 0,316 & 0,935 & 0,107 & 0,163 & 0,218 & 0,955 \\
\hline \multicolumn{10}{|l|}{ Parâmetro cópula } \\
\hline$\theta$ & 1 & 1,129 & 0,179 & 0,272 & 0,865 & 1,136 & 0,125 & 0,204 & 0,820 \\
\hline
\end{tabular}


Tabela C.11: Resumo de 200 replicas com $\theta=1$, censura: $70 \%$ e $\left(J_{0}, J_{1}, J_{2}\right)=(3,3,3)$ segundo a cópula de Clayton. Modelo fixo. (Valor: Verdadeiro valor do parâmetro, Est: média das médias a posteriori, SD: média dos desvios padrão a posteriori, RMSE: média das raízes quadradas do erro quadrático médio, CP: probabilidade de cobertura do HPD de 95\%)

\begin{tabular}{|c|c|c|c|c|c|c|c|c|c|}
\hline \multirow[b]{2}{*}{ Parâmetro } & \multirow[b]{2}{*}{ Valor } & \multicolumn{4}{|c|}{$\bar{N} N=500$} & \multicolumn{4}{|c|}{$N=1000$} \\
\hline & & Est & SD & RMSE & $\mathrm{CP}$ & Est & $\mathrm{SD}$ & RMSE & $\mathrm{CP}$ \\
\hline \multicolumn{10}{|c|}{ Função de risco } \\
\hline$\lambda_{0}$ & 1 & 1,173 & 0,428 & 0,599 & 0,910 & 1,137 & 0,289 & 0,412 & 0,930 \\
\hline$\lambda_{0}$ & 1 & 1,185 & 0,441 & 0,610 & 0,905 & 1,140 & 0,295 & 0,414 & 0,940 \\
\hline$\lambda_{0}$ & 1 & 0,287 & 0,116 & 0,726 & 0,040 & 0,259 & 0,072 & 0,746 & 0,000 \\
\hline$\lambda_{1}$ & 5 & 4,835 & 0,685 & 0,952 & 0,920 & 4,867 & 0,485 & 0,672 & 0,930 \\
\hline$\lambda_{1}$ & 5 & 4,786 & 0,634 & 0,895 & 0,930 & 4,826 & 0,450 & 0,649 & 0,915 \\
\hline$\lambda_{1}$ & 5 & 4,923 & 0,688 & 0,964 & 0,920 & 4,805 & 0,473 & 0,667 & 0,900 \\
\hline$\lambda_{2}$ & 1 & 0,958 & 0,256 & 0,353 & 0,925 & 0,962 & 0,179 & 0,253 & 0,920 \\
\hline$\lambda_{2}$ & 1 & 0,924 & 0,248 & 0,343 & 0,930 & 0,887 & 0,166 & 0,255 & 0,845 \\
\hline$\lambda_{2}$ & 1 & 0,301 & 0,083 & 0,704 & 0,000 & 0,293 & 0,056 & 0,710 & 0,000 \\
\hline \multicolumn{10}{|l|}{ Modelo $T_{D}$} \\
\hline$\beta_{0}$ & -1 & $-1,319$ & 0,327 & 0,528 & 0,835 & $-1,289$ & 0,226 & 0,411 & 0,745 \\
\hline$\gamma_{01}$ & 1 & 1,311 & 0,186 & 0,376 & 0,650 & 1,271 & 0,127 & 0,306 & 0,450 \\
\hline$\gamma_{02}$ & 0,2 & 0,258 & 0,311 & 0,429 & 0,935 & 0,239 & 0,213 & 0,305 & 0,935 \\
\hline \multicolumn{10}{|l|}{ Modelo $T_{E}$} \\
\hline$\beta_{1}$ & $-0,5$ & $-0,485$ & 0,125 & 0,173 & 0,920 & $-0,498$ & 0,087 & 0,114 & 0,960 \\
\hline$\gamma_{11}$ & 1 & 1,008 & 0,079 & 0,108 & 0,945 & 1,000 & 0,055 & 0,077 & 0,905 \\
\hline$\gamma_{12}$ & 0 & $-0,001$ & 0,119 & 0,159 & 0,955 & $-0,004$ & 0,084 & 0,109 & 0,965 \\
\hline \multicolumn{10}{|l|}{ Modelo $T_{G}$} \\
\hline$\beta_{21}$ & $-0,3$ & $-0,458$ & 0,227 & 0,333 & 0,920 & $-0,459$ & 0,158 & 0,253 & 0,820 \\
\hline$\beta_{22}$ & $-0,5$ & $-0,633$ & 0,228 & 0,331 & 0,910 & $-0,640$ & 0,159 & 0,247 & 0,870 \\
\hline$\gamma_{21}$ & $-0,5$ & $-0,480$ & 0,109 & 0,151 & 0,925 & $-0,483$ & 0,076 & 0,104 & 0,920 \\
\hline$\gamma_{22}$ & 0,5 & 0,645 & 0,210 & 0,307 & 0,895 & 0,641 & 0,146 & 0,242 & 0,815 \\
\hline \multicolumn{10}{|l|}{ Modelo logito } \\
\hline$\alpha_{0}$ & 1,6 & 1,721 & 0,249 & 0,338 & 0,915 & 1,719 & 0,175 & 0,260 & 0,865 \\
\hline$\alpha_{1}$ & $-1,8$ & $-1,901$ & 0,257 & 0,359 & 0,915 & $-1,914$ & 0,182 & 0,260 & 0,940 \\
\hline$\alpha_{2}$ & 1 & 1,119 & 0,146 & 0,223 & 0,855 & 1,097 & 0,102 & 0,162 & 0,830 \\
\hline$\alpha_{3}$ & 0,1 & 0,073 & 0,243 & 0,335 & 0,955 & 0,090 & 0,170 & 0,229 & 0,955 \\
\hline \multicolumn{10}{|c|}{ Parâmetro cópula } \\
\hline$\theta$ & 1 & 1,505 & 0,274 & 0,588 & 0,485 & 1,529 & 0,193 & 0,566 & 0,135 \\
\hline
\end{tabular}



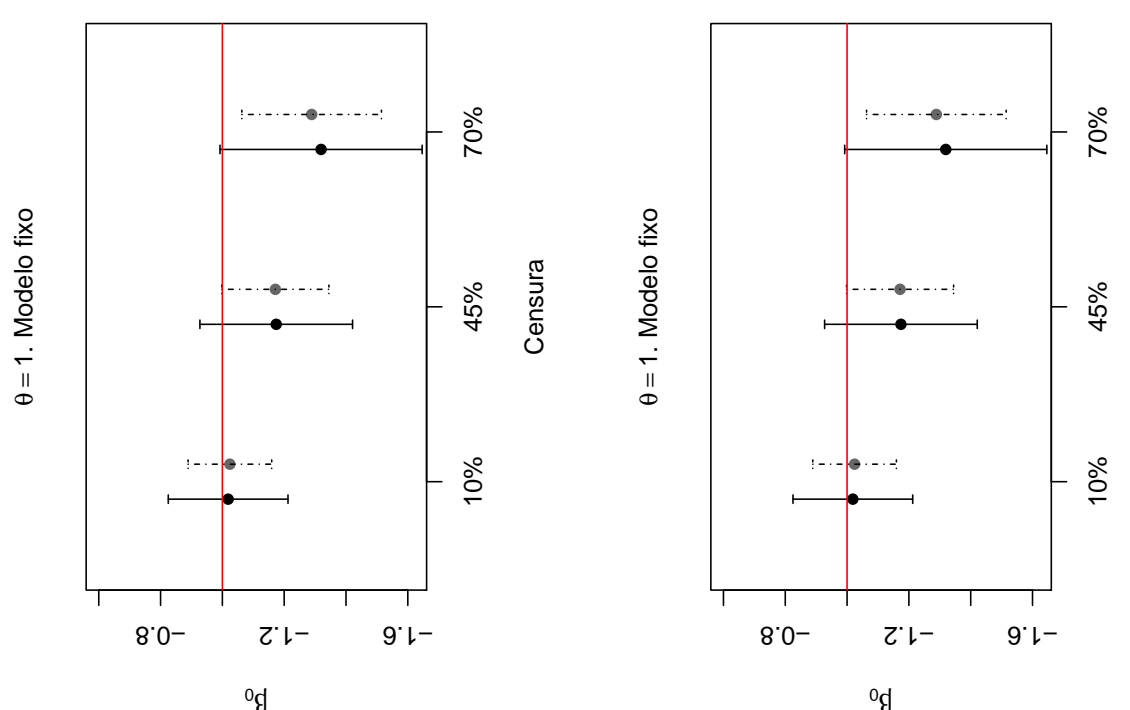

${ }^{\circ} \mathrm{g}$
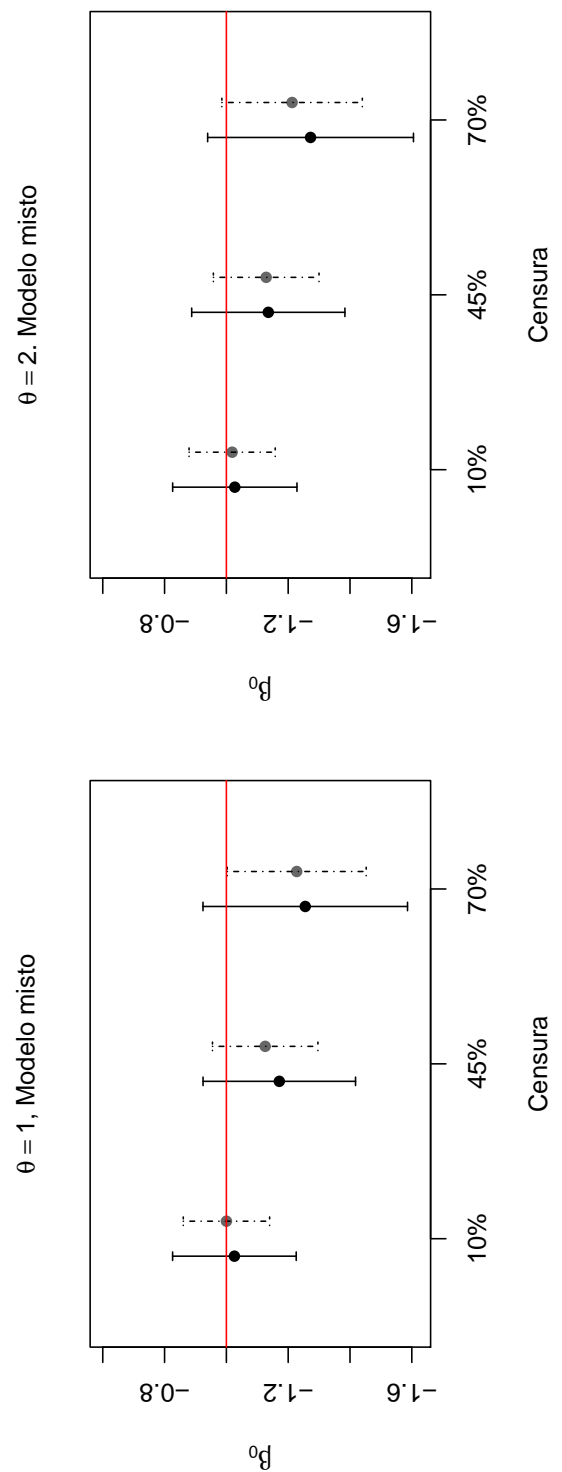
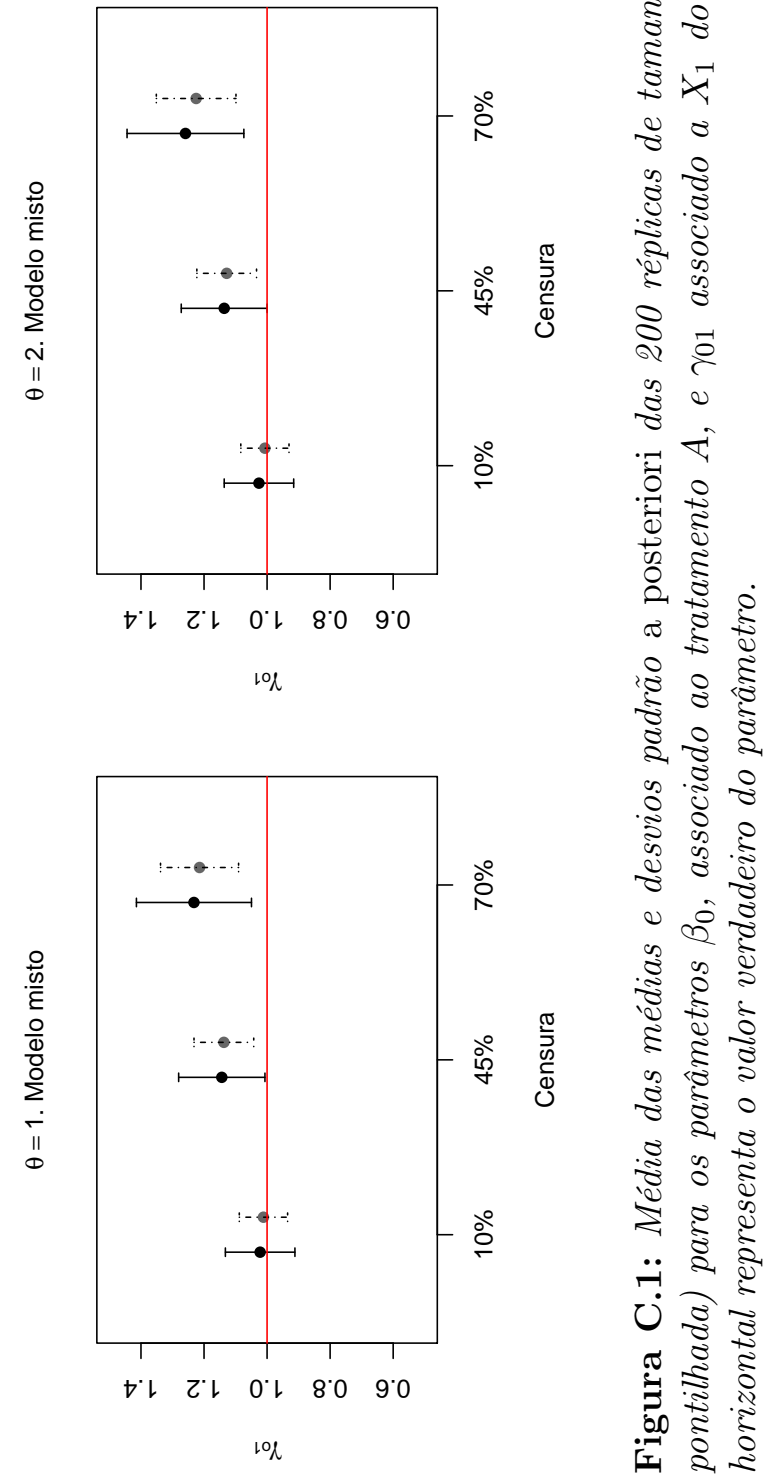


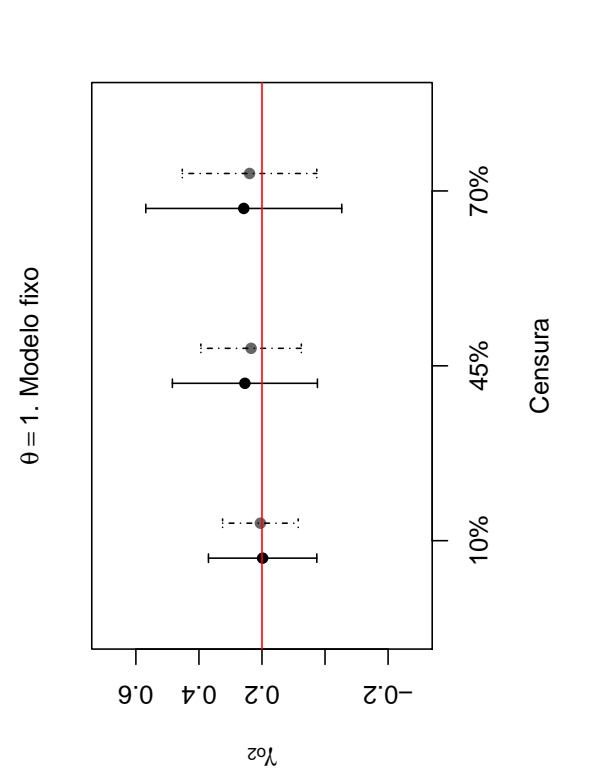

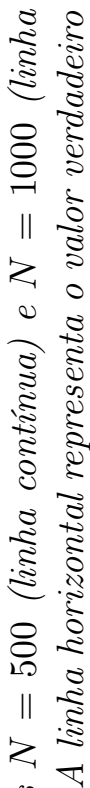

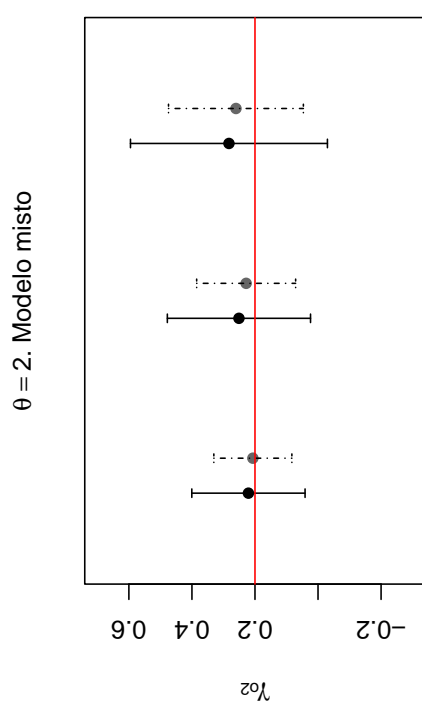

点

हु

요 $\quad \frac{\pi}{8}$

离

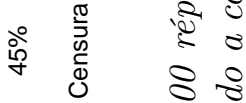

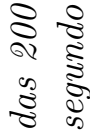

웅

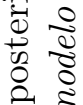

है

\& 8

is 8

है

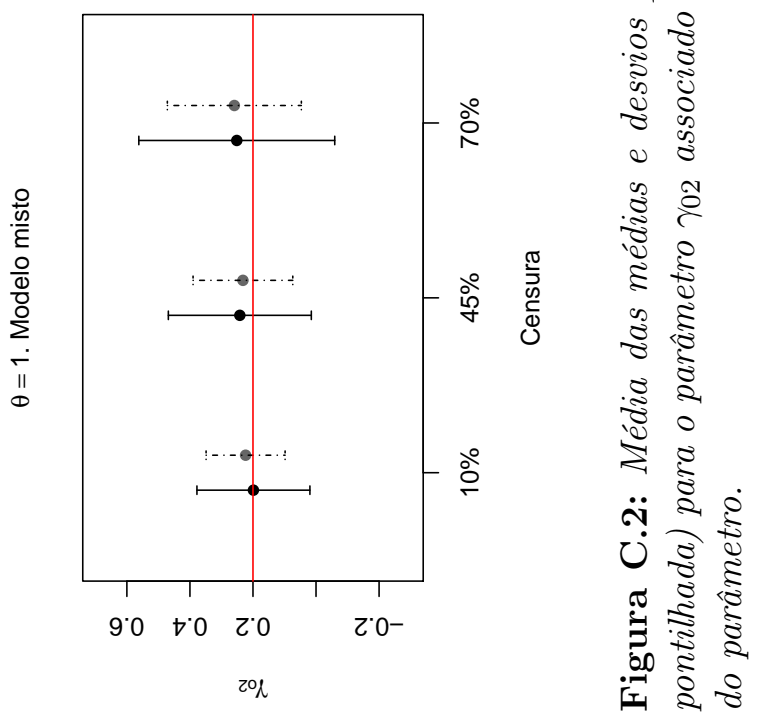



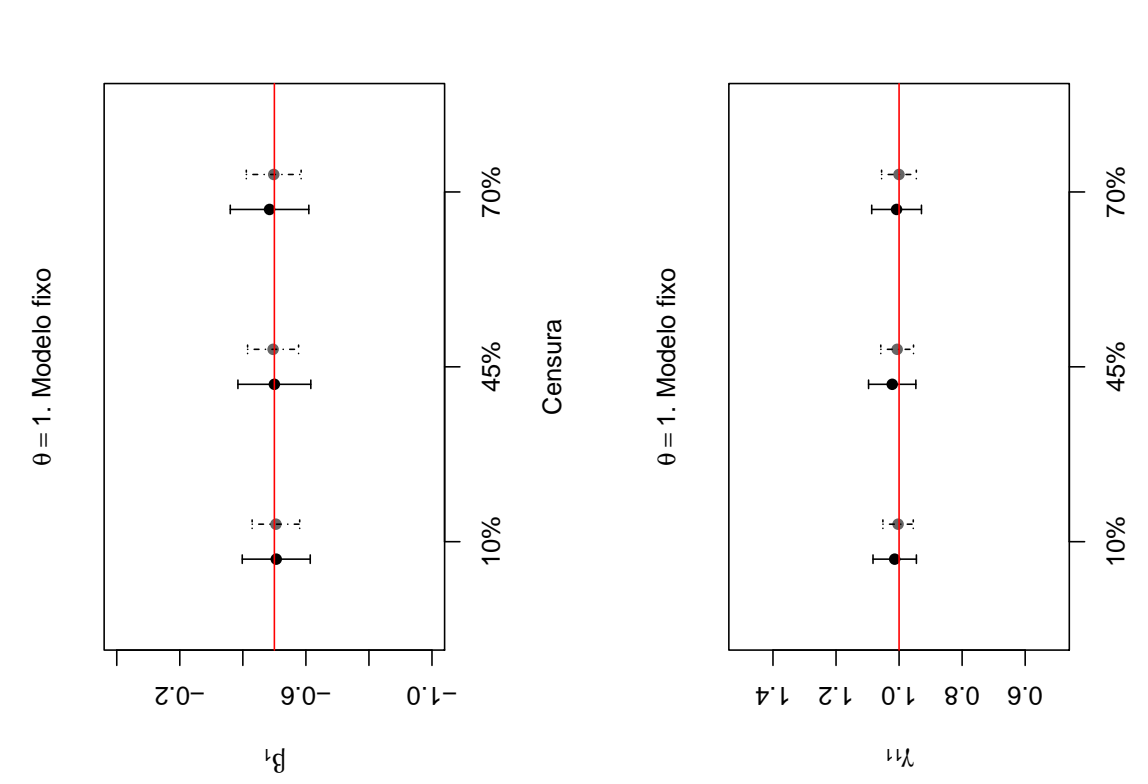

离.
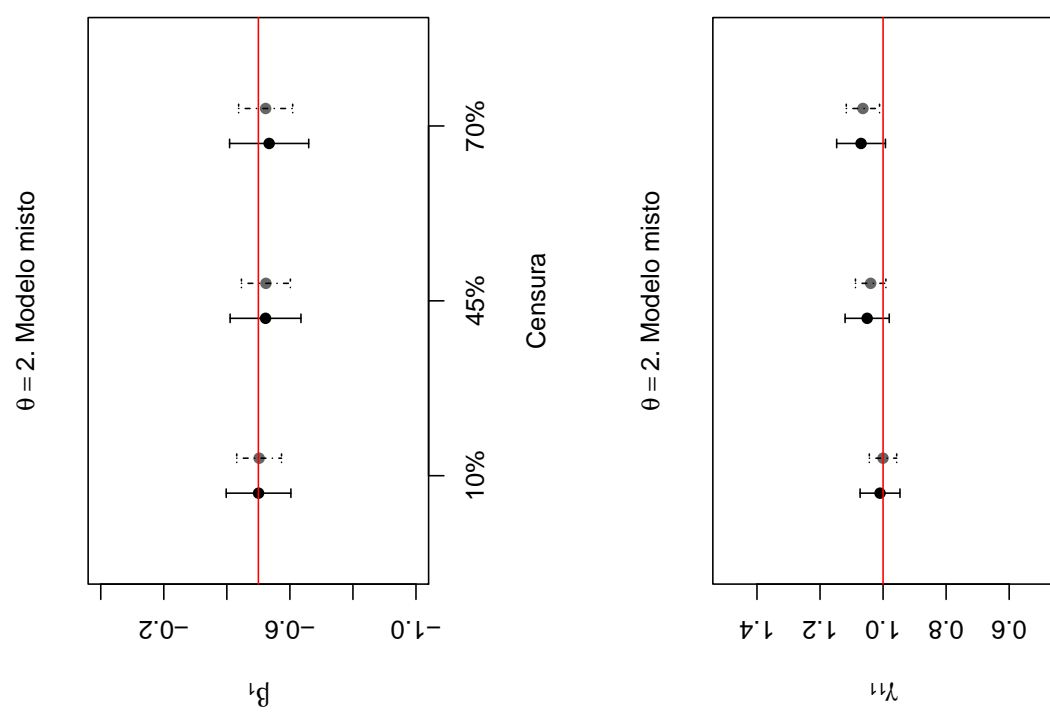

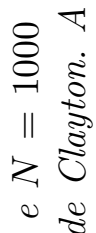

$\frac{\sqrt[0]{7}}{\bar{m}}$

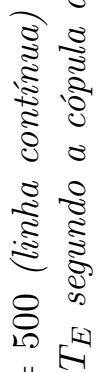

$z_{0} \frac{\Xi}{\mathbb{\Xi}}$

क ह

है ?

हैं

$\approx 0$

क :

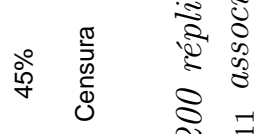

बे

30

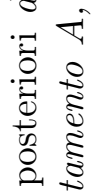

$\pi$ 胥

\& \&

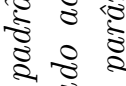
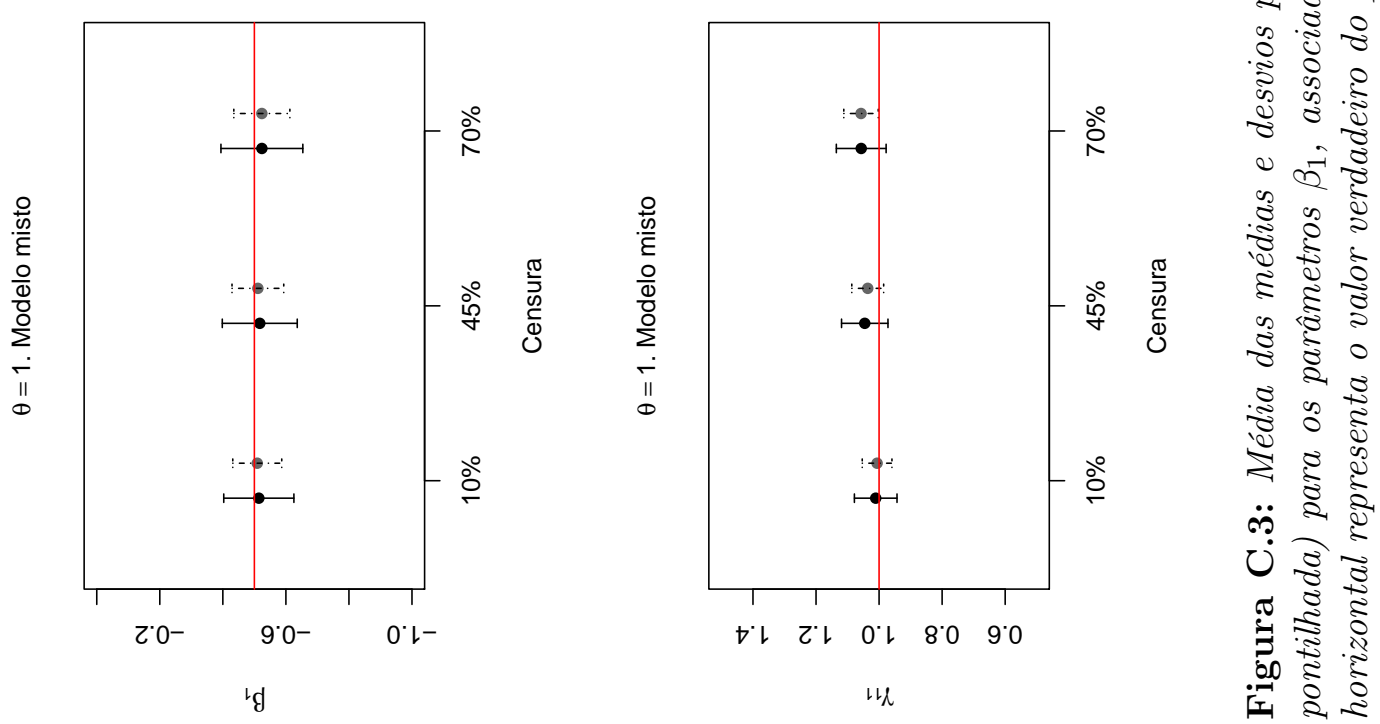


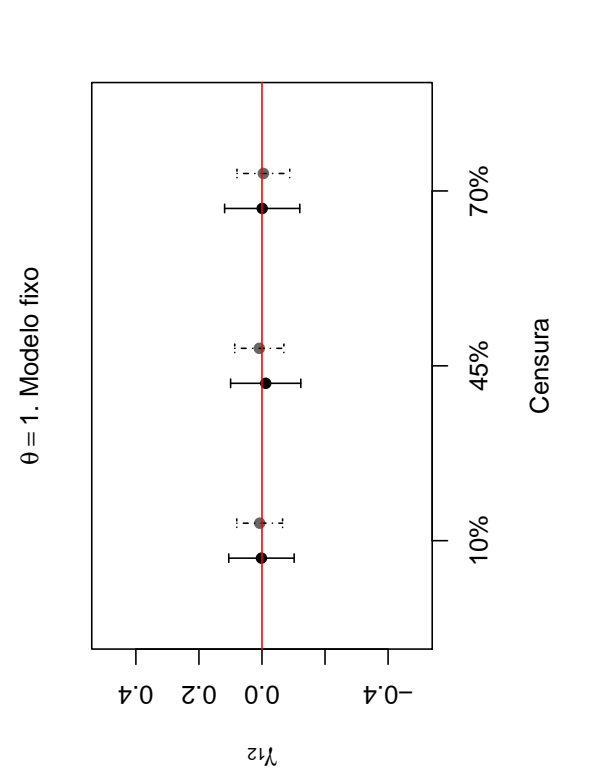

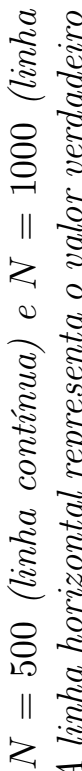

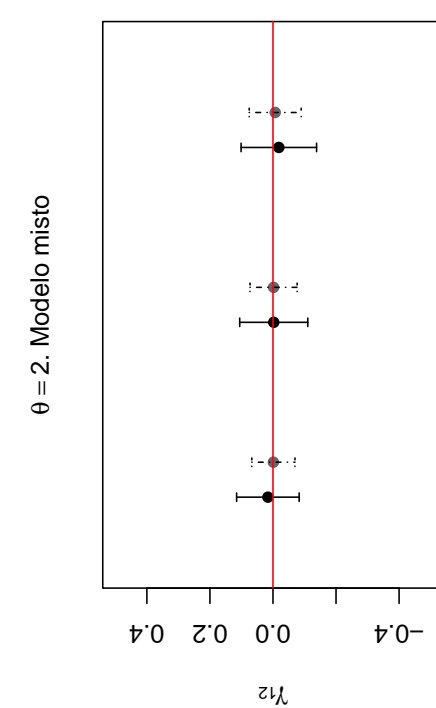

8

के ह

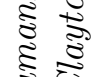

छे

离

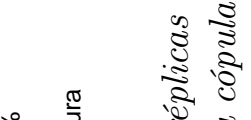

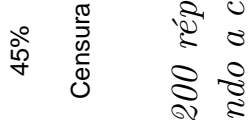

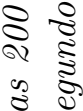

के के

号

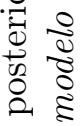

$\approx \%$

है

造,

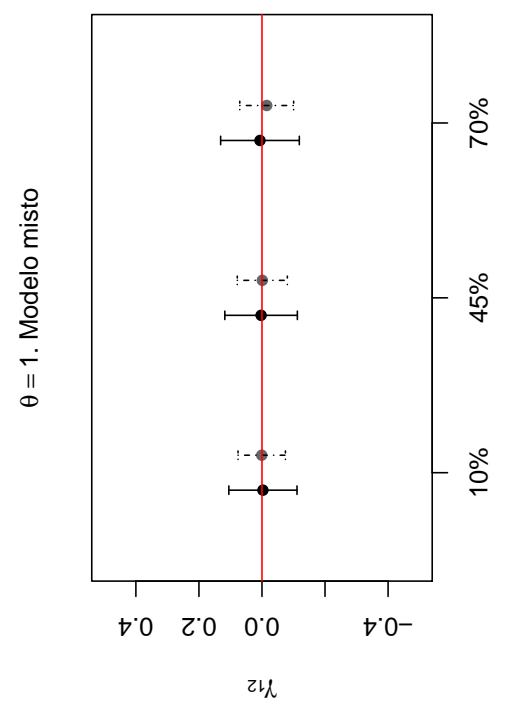

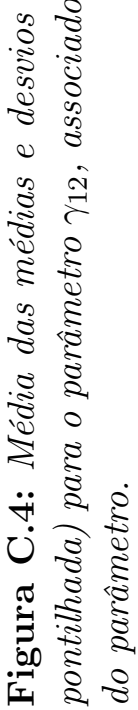




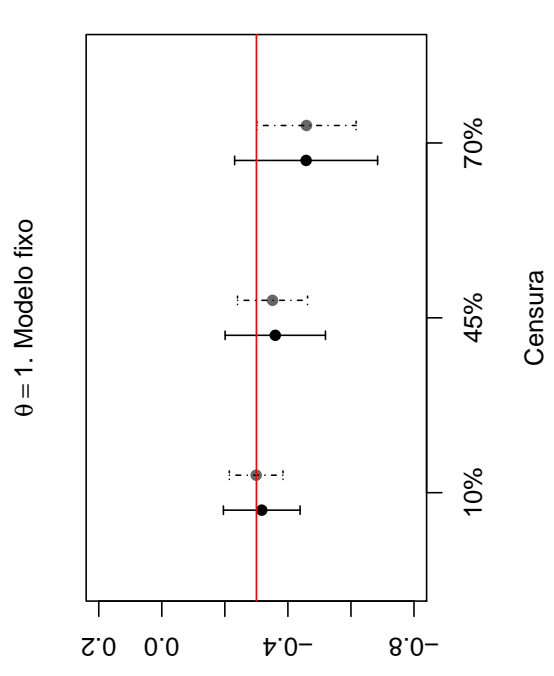

${ }^{12} \mathrm{~g}$

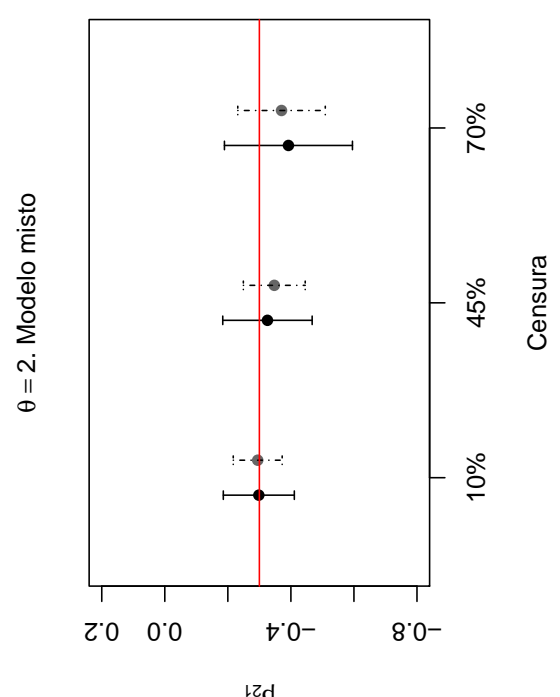

$\operatorname{lrg}$

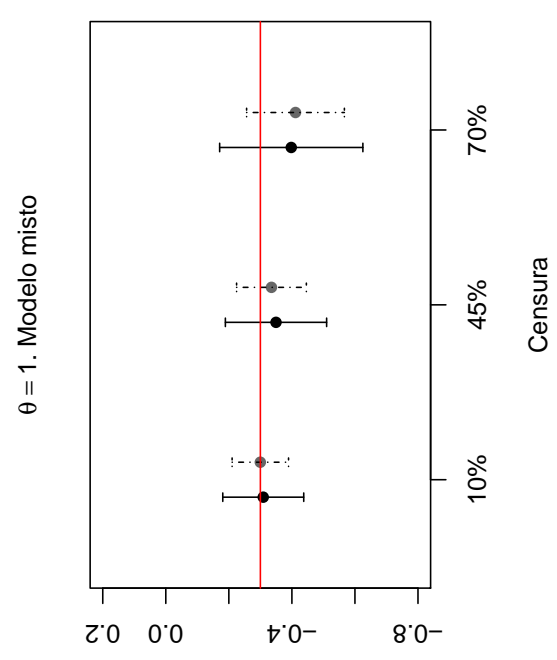

${ }^{2} \mathrm{~g}$

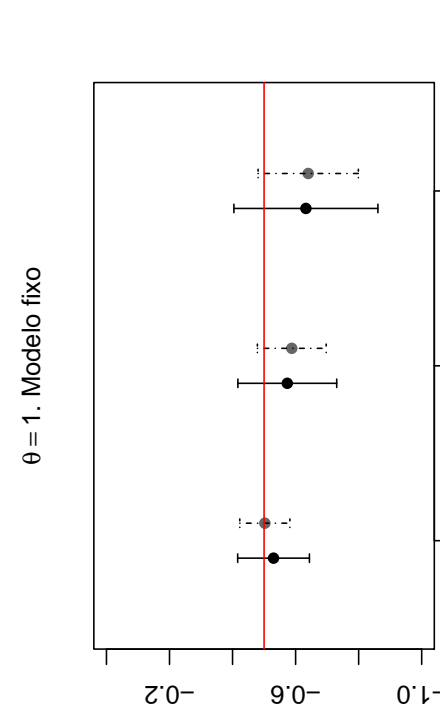

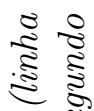

80

1 웅

$z$ ह
0 ह

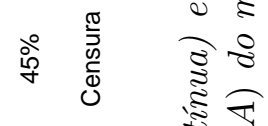

$2 z \mathrm{~g}$

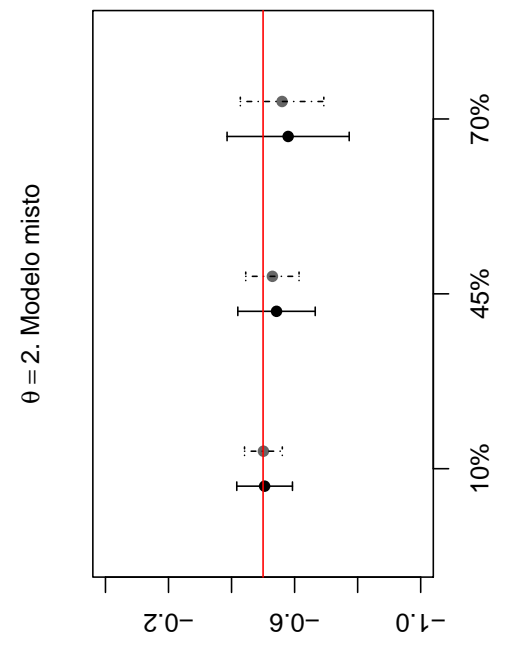

$z z g$

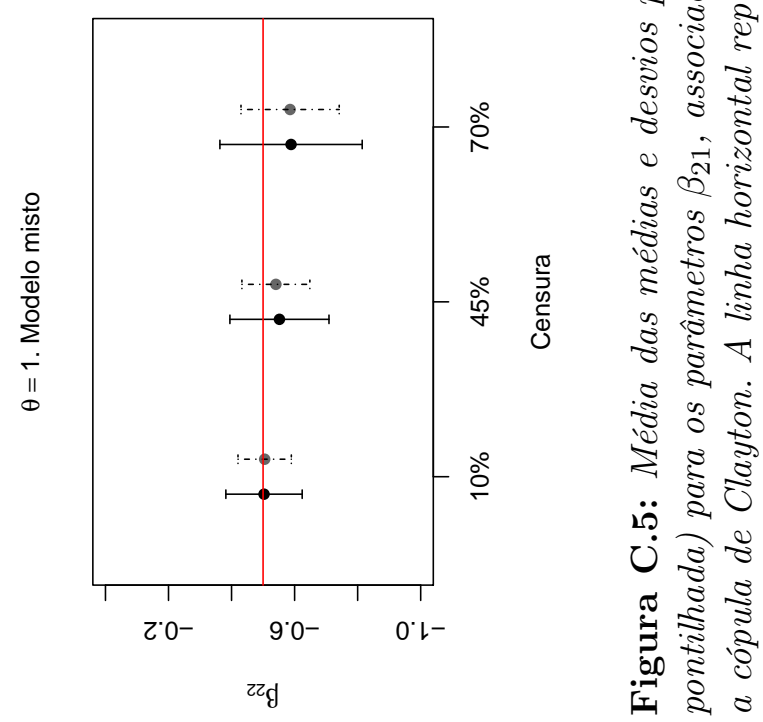




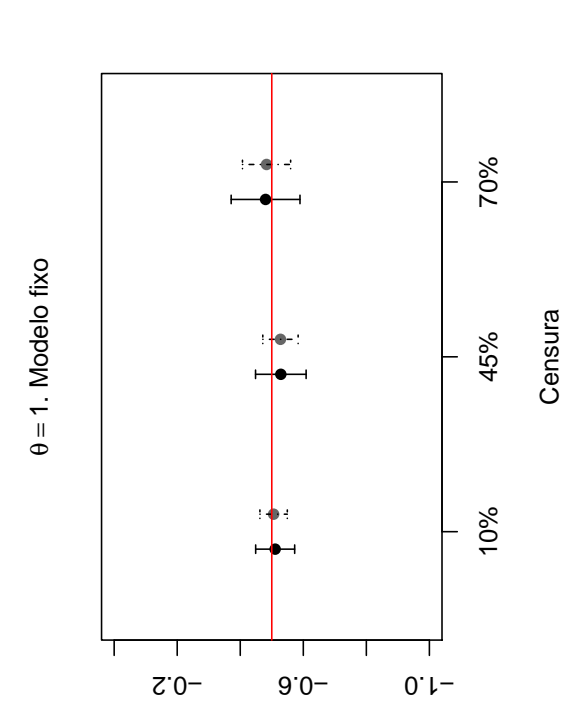

ז2

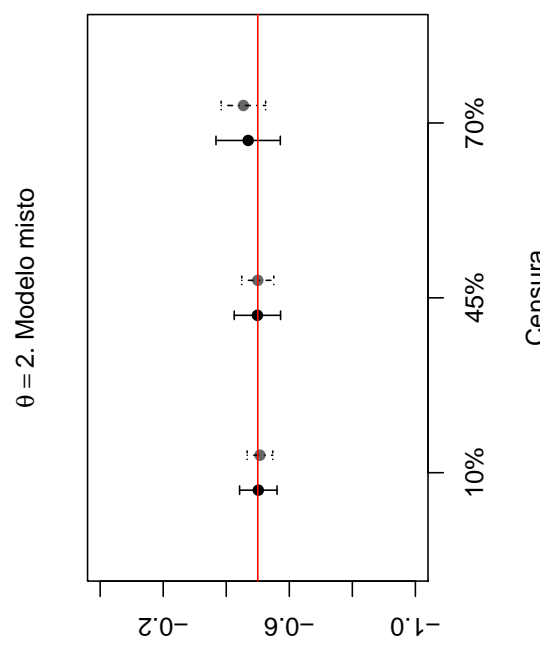

12

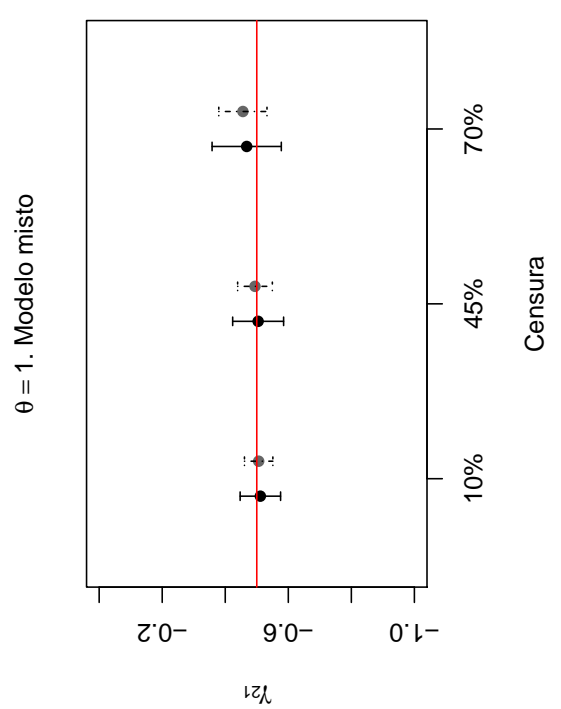

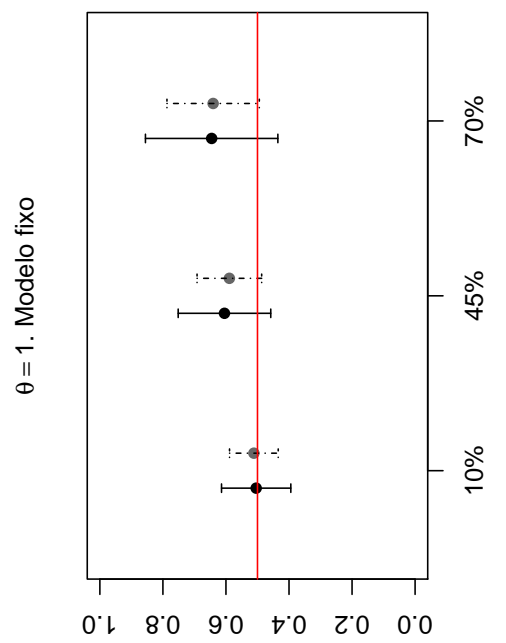

2टน

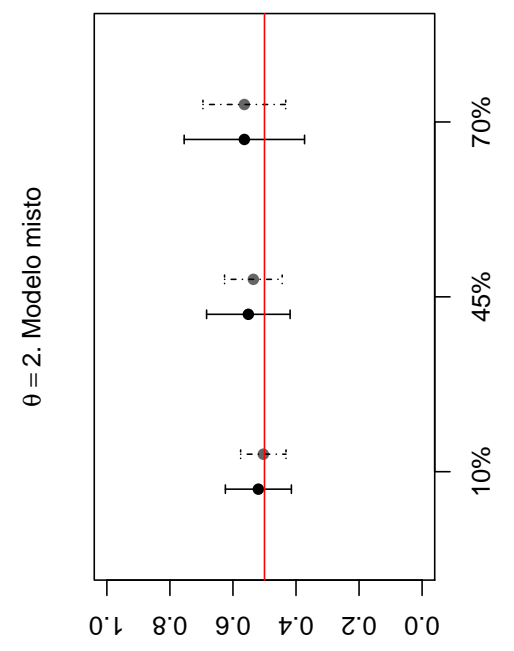

22/

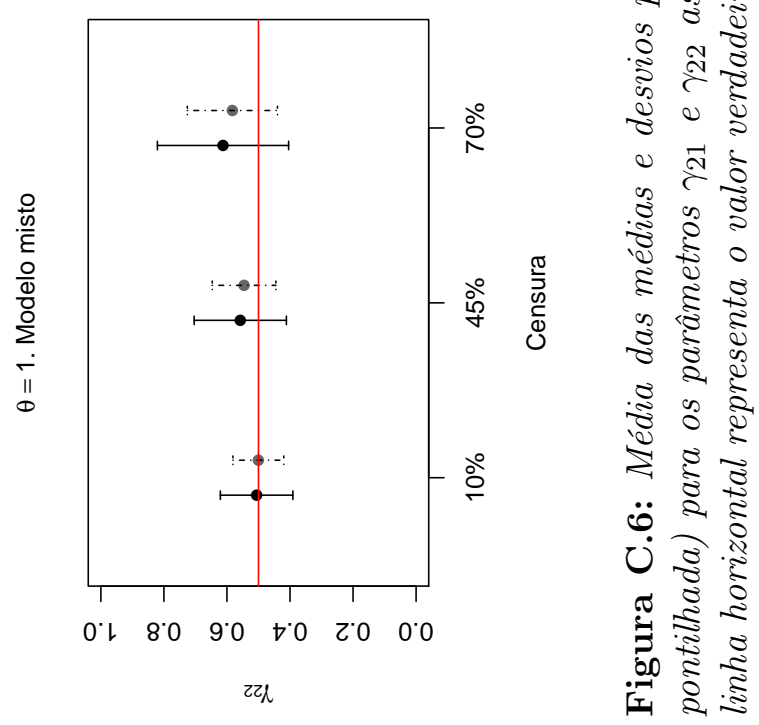

$\stackrel{2}{2}$

.્ิ

\&

$11 \frac{8}{8}$

z $\frac{0}{3}$

ग)

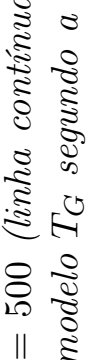

$z \stackrel{8}{8}$

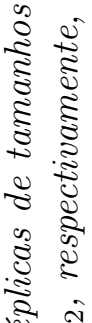

एँ

จ

o

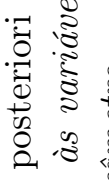

ช 3

요

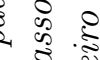

กิ

( )

- ำ

0 5

ह

है,

0 \&

จ

2

용

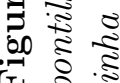




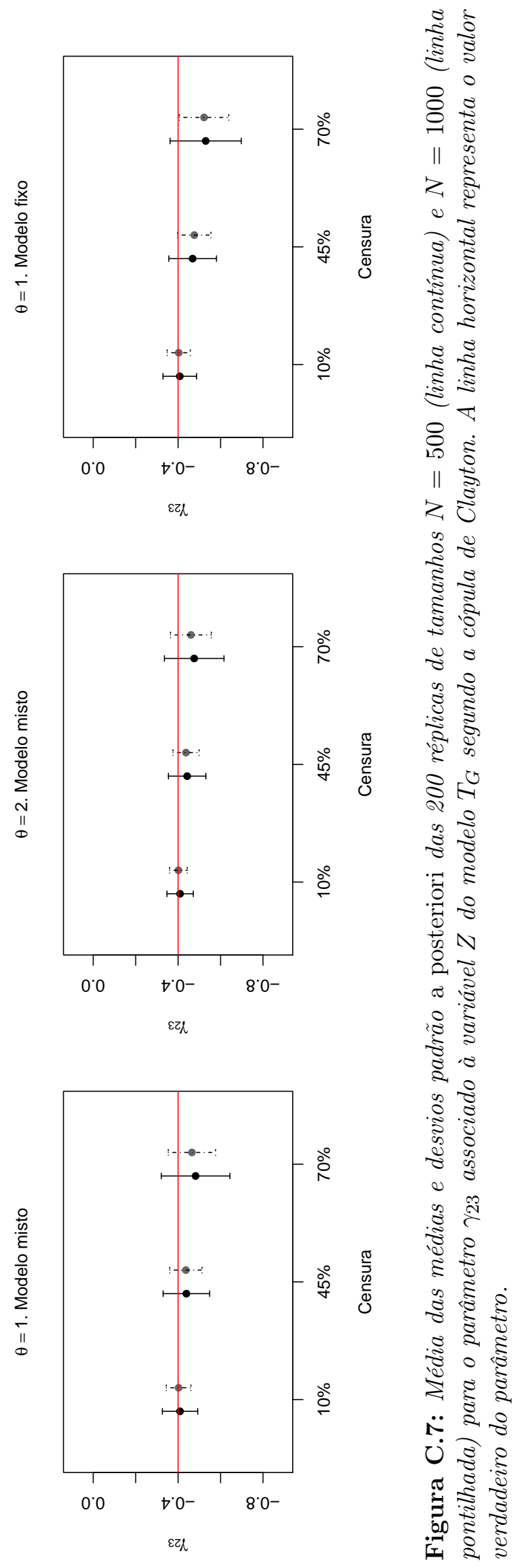




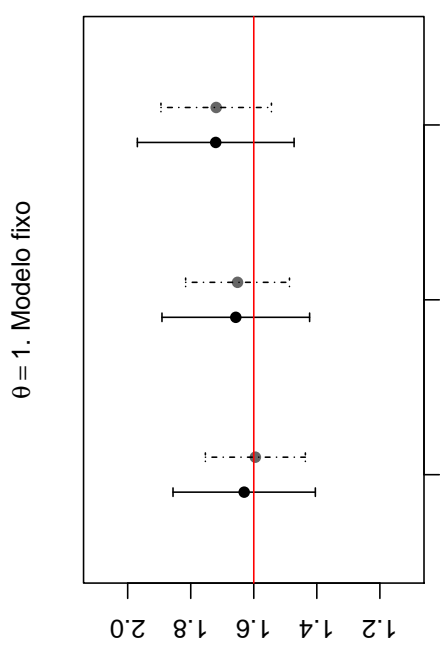

$0 x$
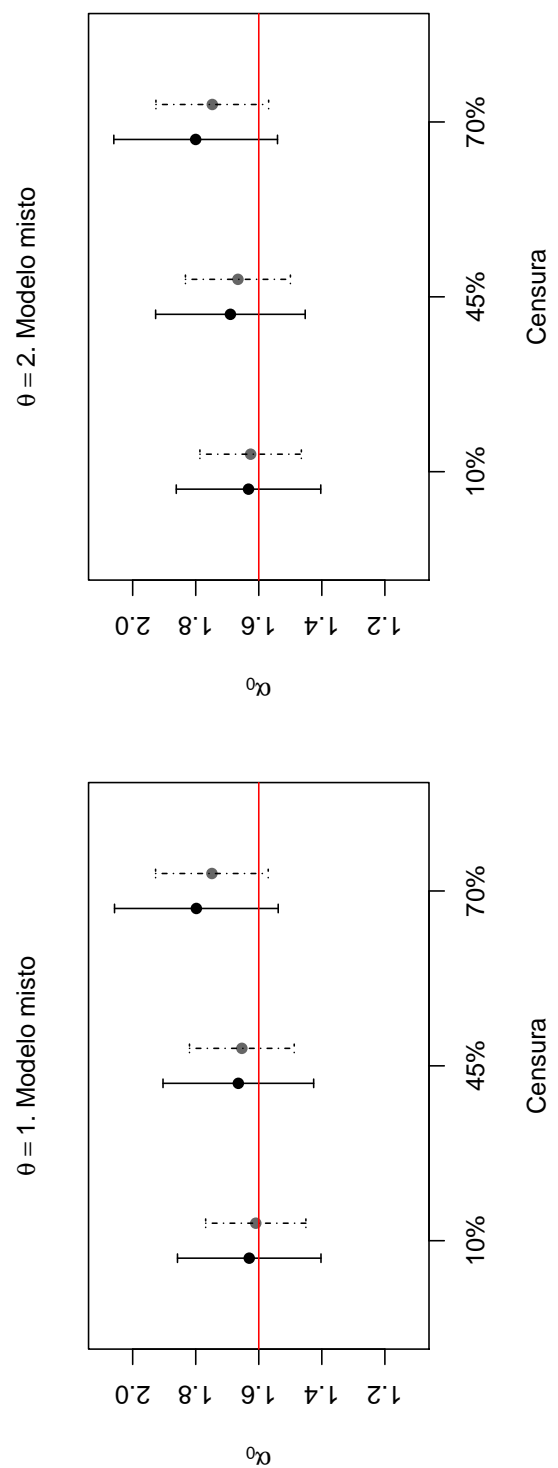

ஓ̊

@

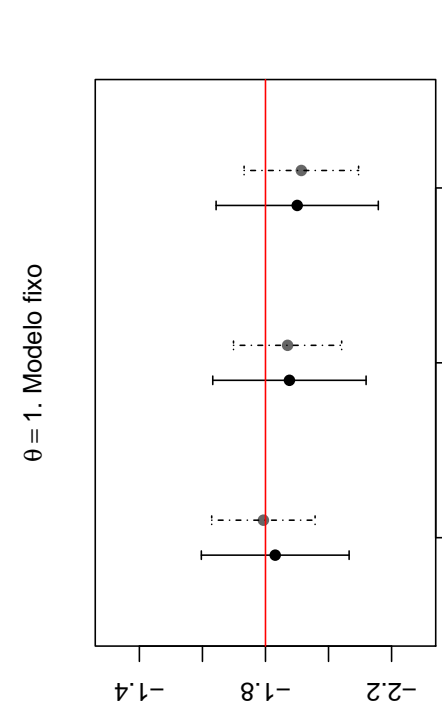

हี :

$t_{0}$
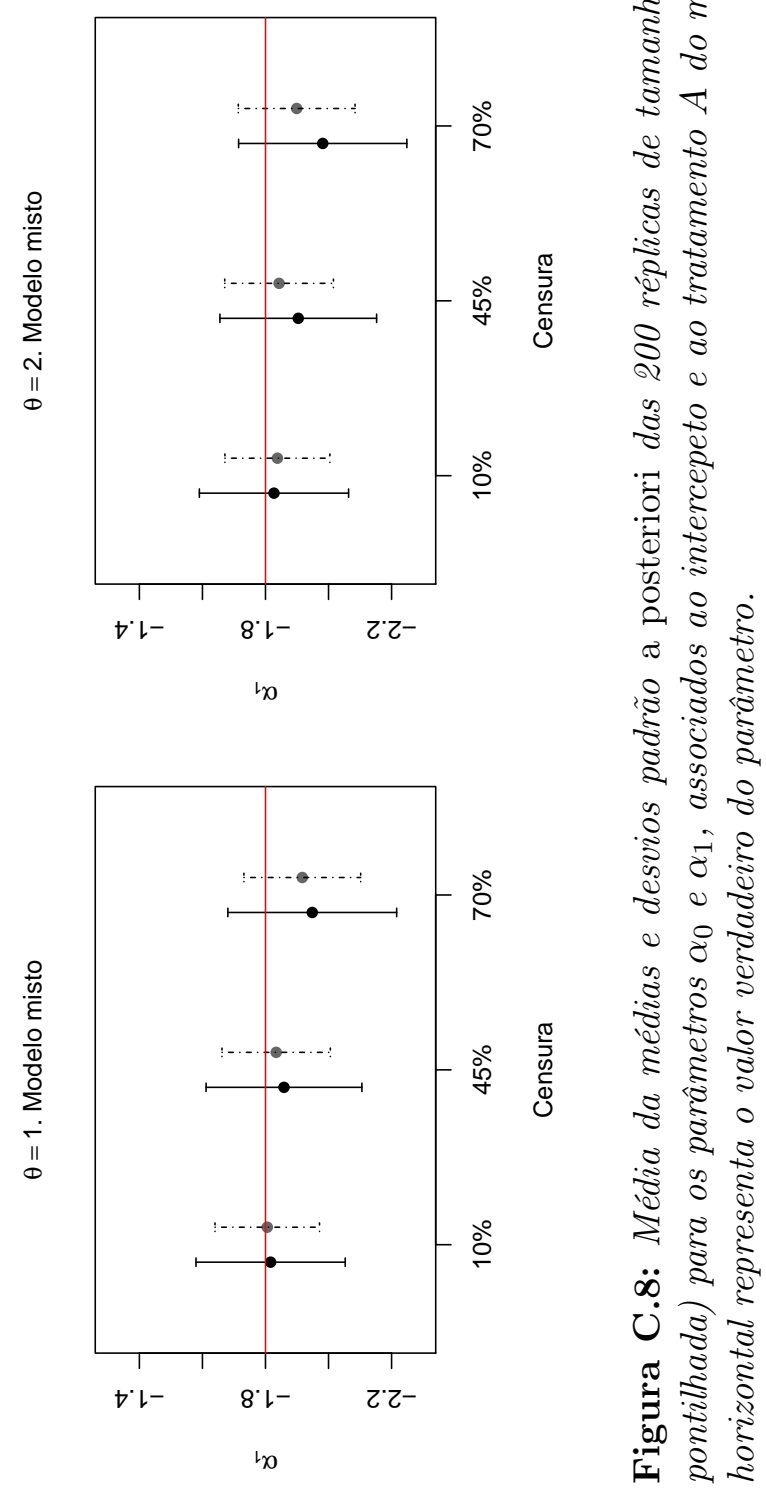

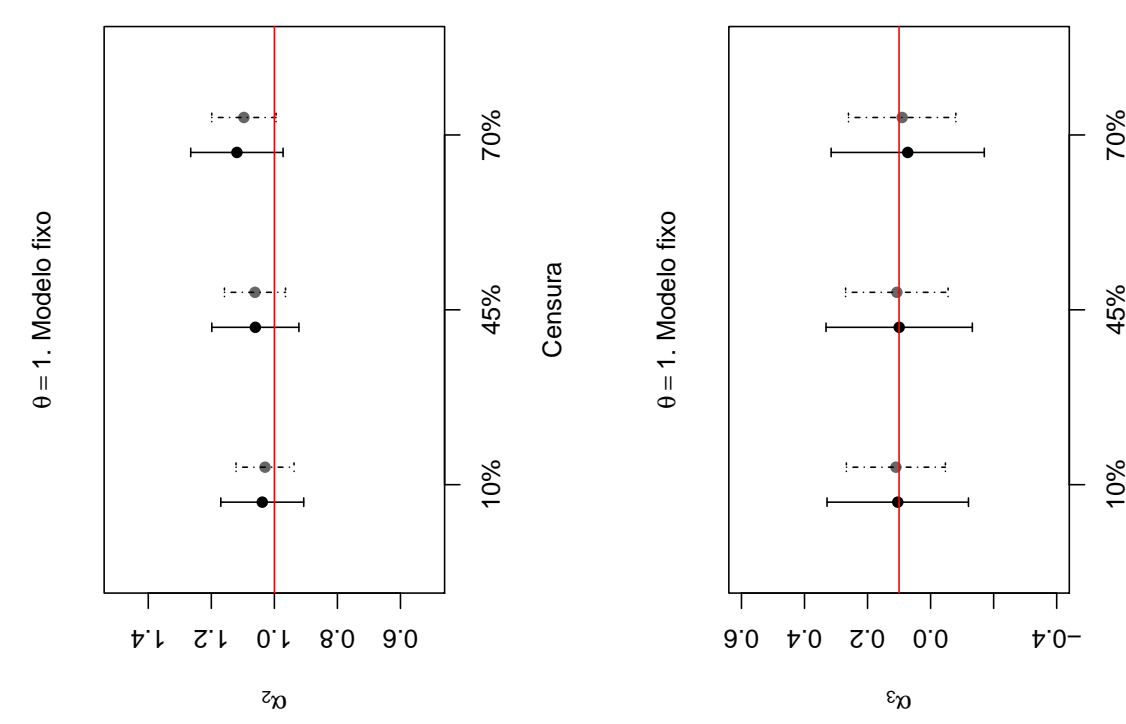

:
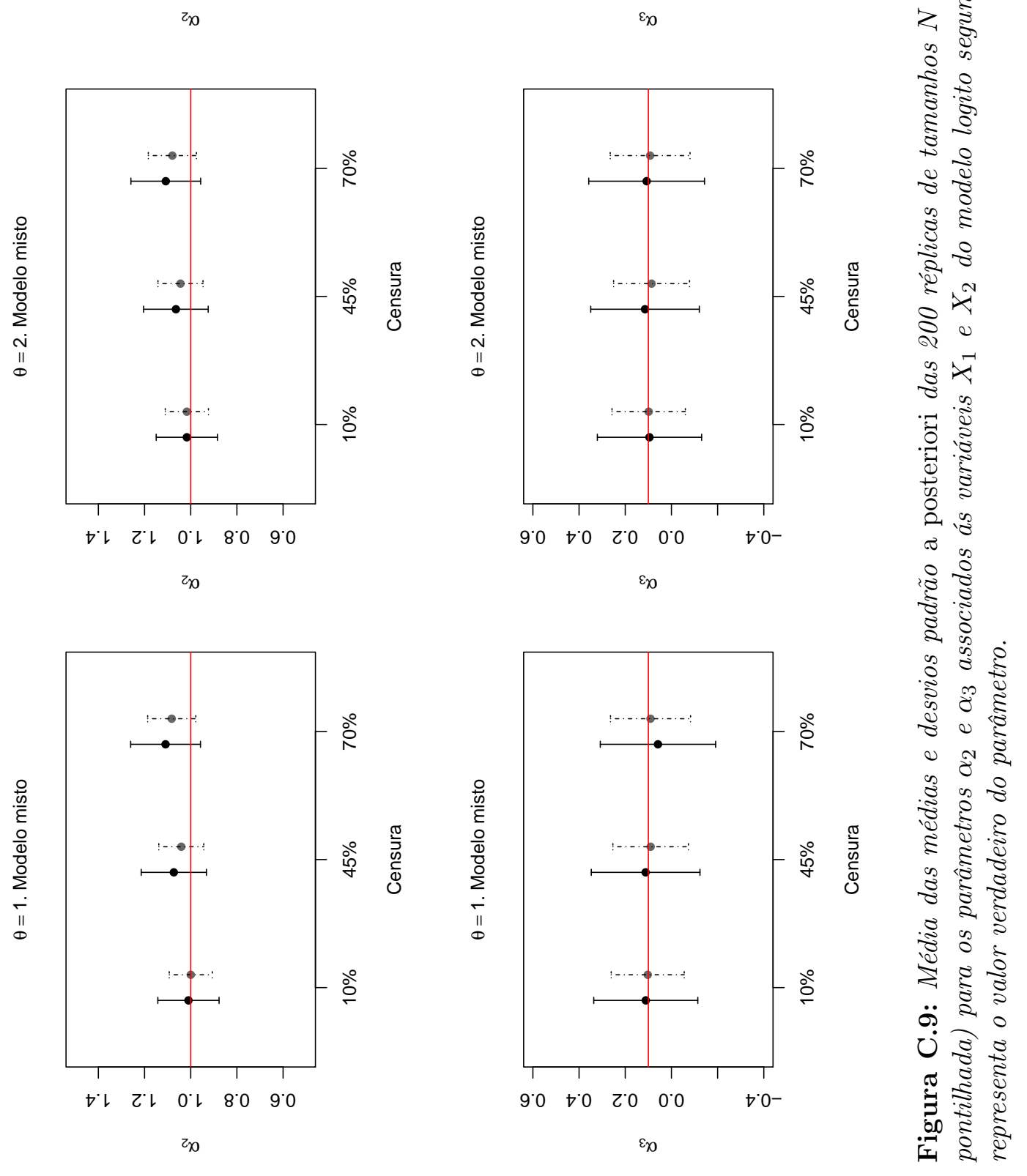


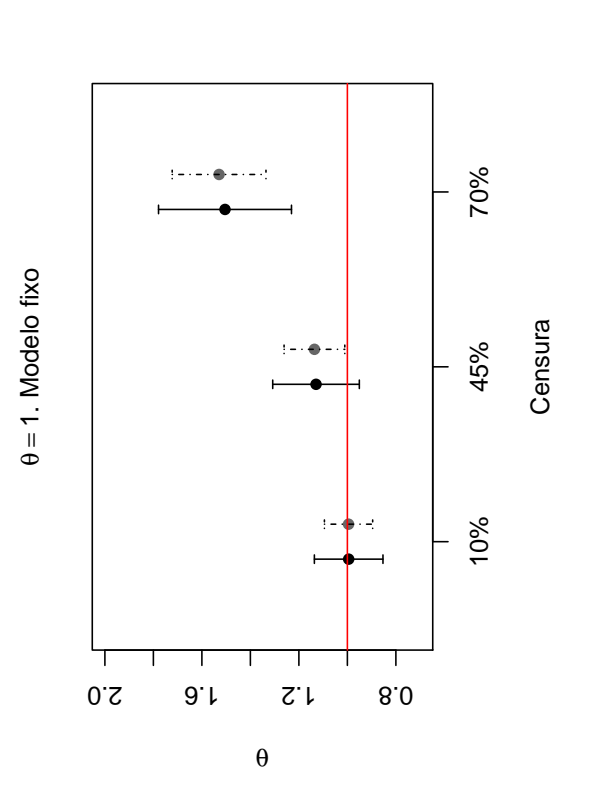

芯

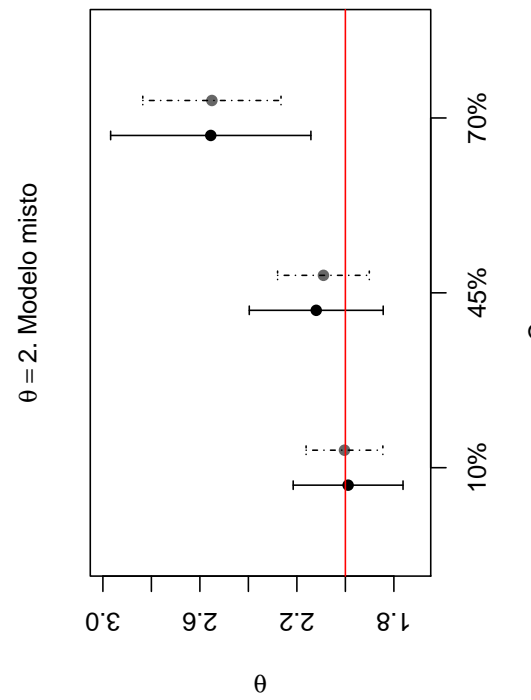

2

8
8
0
-1
11
$z$
7
0

z

$\begin{array}{ll}0 & 8 \\ 2 & 0\end{array}$

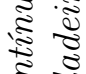

8 है

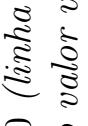

용요

11 है

$z_{1}$
के है
0

है

है

จ

离芯

จे

क ज्ञ

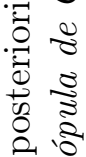

$\pi 0$

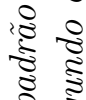

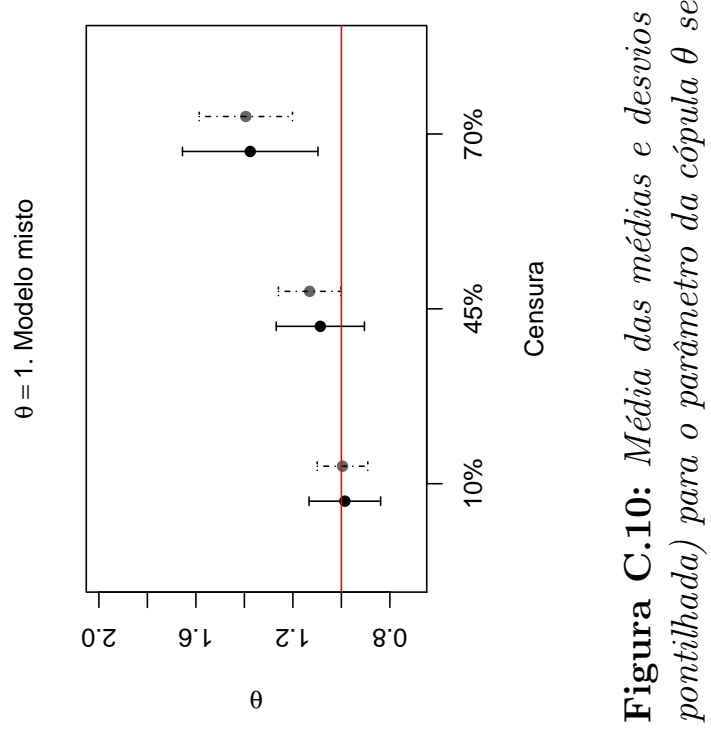




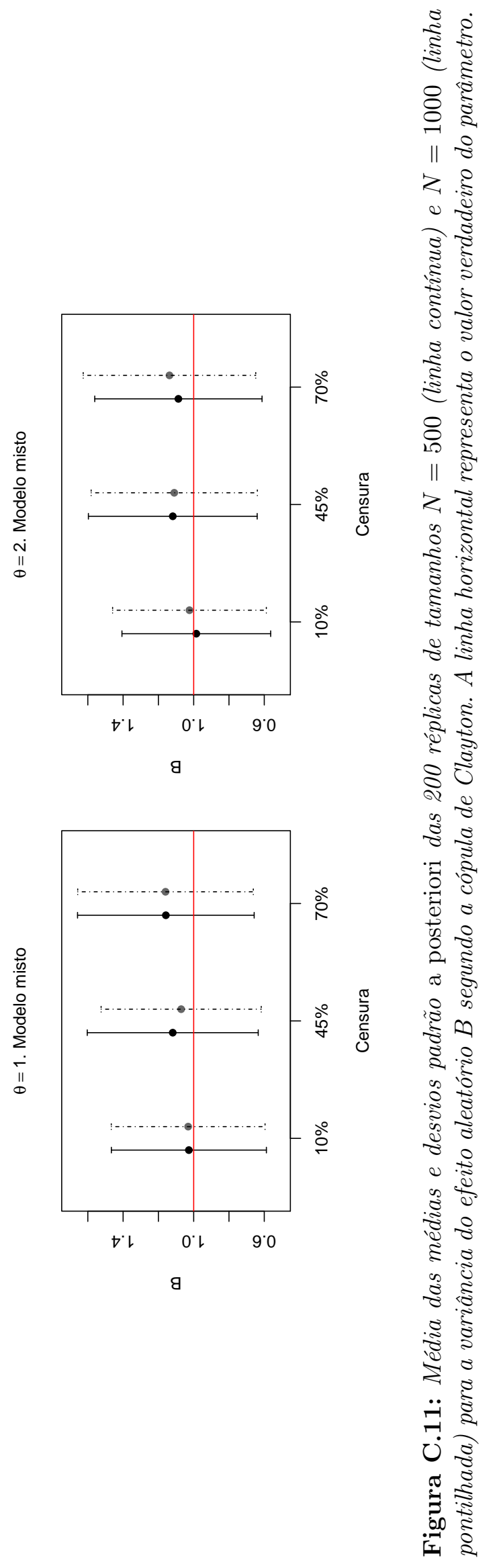


Apêndice

\section{Figuras das aplicações}

Para facilitar a leitura do Capítulo 5 correspondente ás aplicações, neste apêndice são anexadas as figuras obtidas da inferência bayesiana no ajuste da modelagem proposta.

Na Seção D.1 podem ser consultados os gráficos com as funções densidade a posteriori (histogramas), traços (sequencia dos valores simulados MCMC) e funções de autocorrelação para os parâmetros estimados do TMO.

Na Seção D.2 encontram-se os respectivos gráficos do ajuste da modelagem fixa e mista aos dados dos pacientes com IRC. 


\section{D.1 Transplante de medula óssea (TMO)}
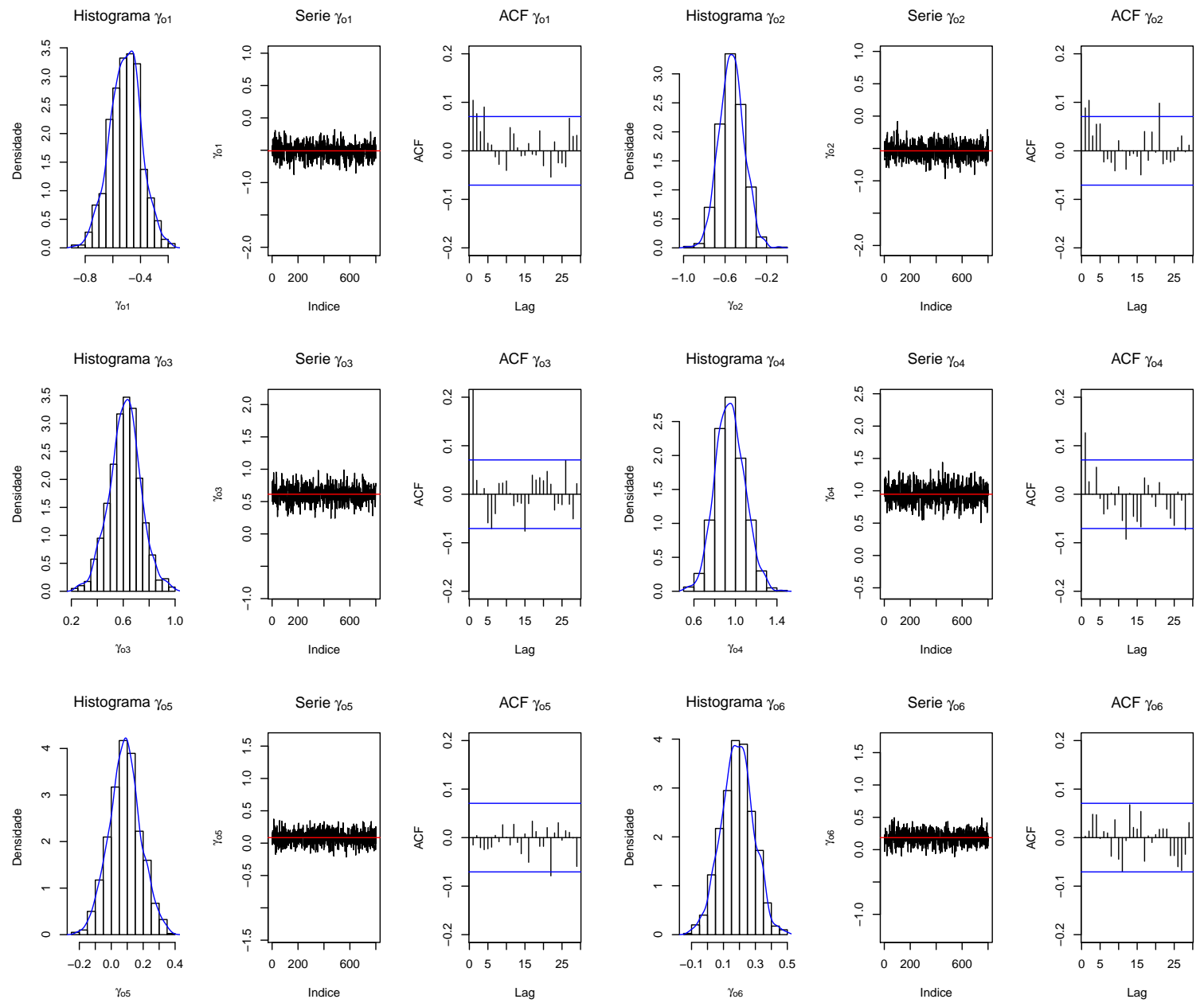

Figura D.1: Densidades a posteriori, traços e ACFs para a componente $T_{D}$ (óbito) do ajuste do modelo fixo com $\left(J_{0}, J_{1}, J_{2}\right)=(15,15,15)$ segundo a cópula de Clayton aos dados de TMO. 

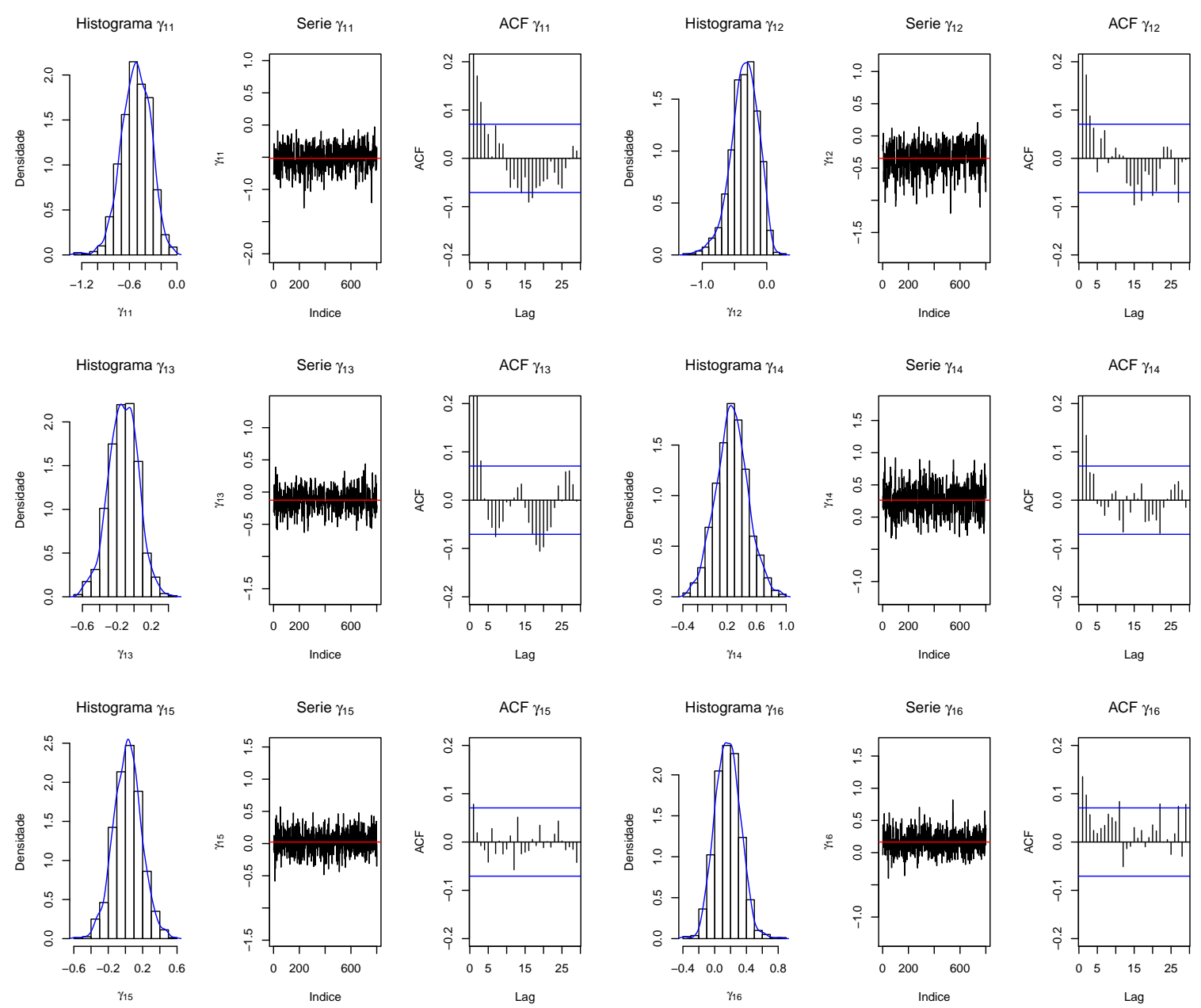

Figura D.2: Densidades a posteriori, traços e ACFs para a componente $T_{E}$ (recaída) do ajuste do modelo fixo com $\left(J_{0}, J_{1}, J_{2}\right)=(15,15,15)$ segundo a cópula de Clayton aos dados de TMO. 

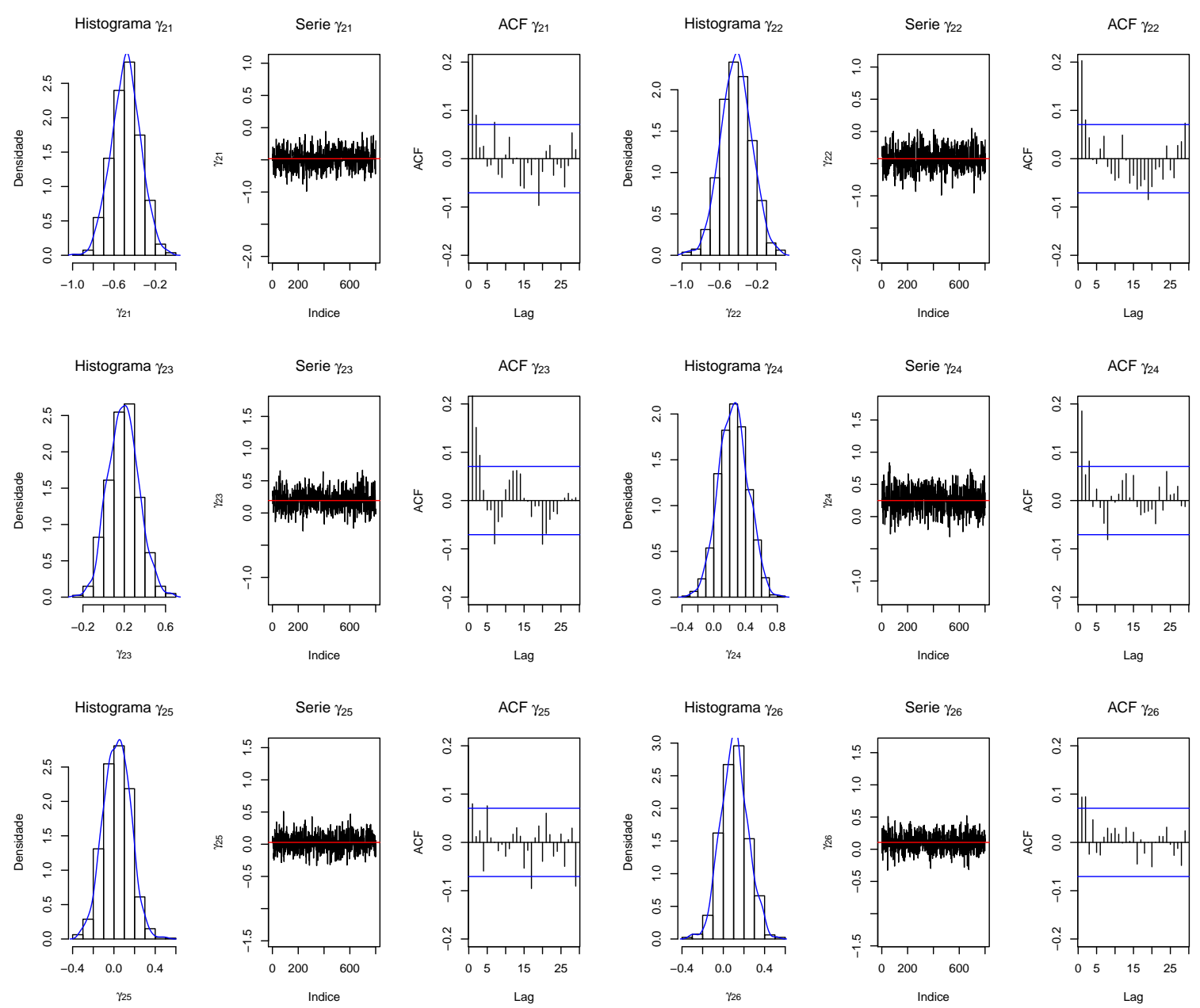

Figura D.3: Densidades a posteriori, traços e ACFs para a componente $T_{G}$ (óbito após recaída) do ajuste do modelo fixo com $\left(J_{0}, J_{1}, J_{2}\right)=(15,15,15)$ segundo a cópula de Clayton aos dados de TMO. 

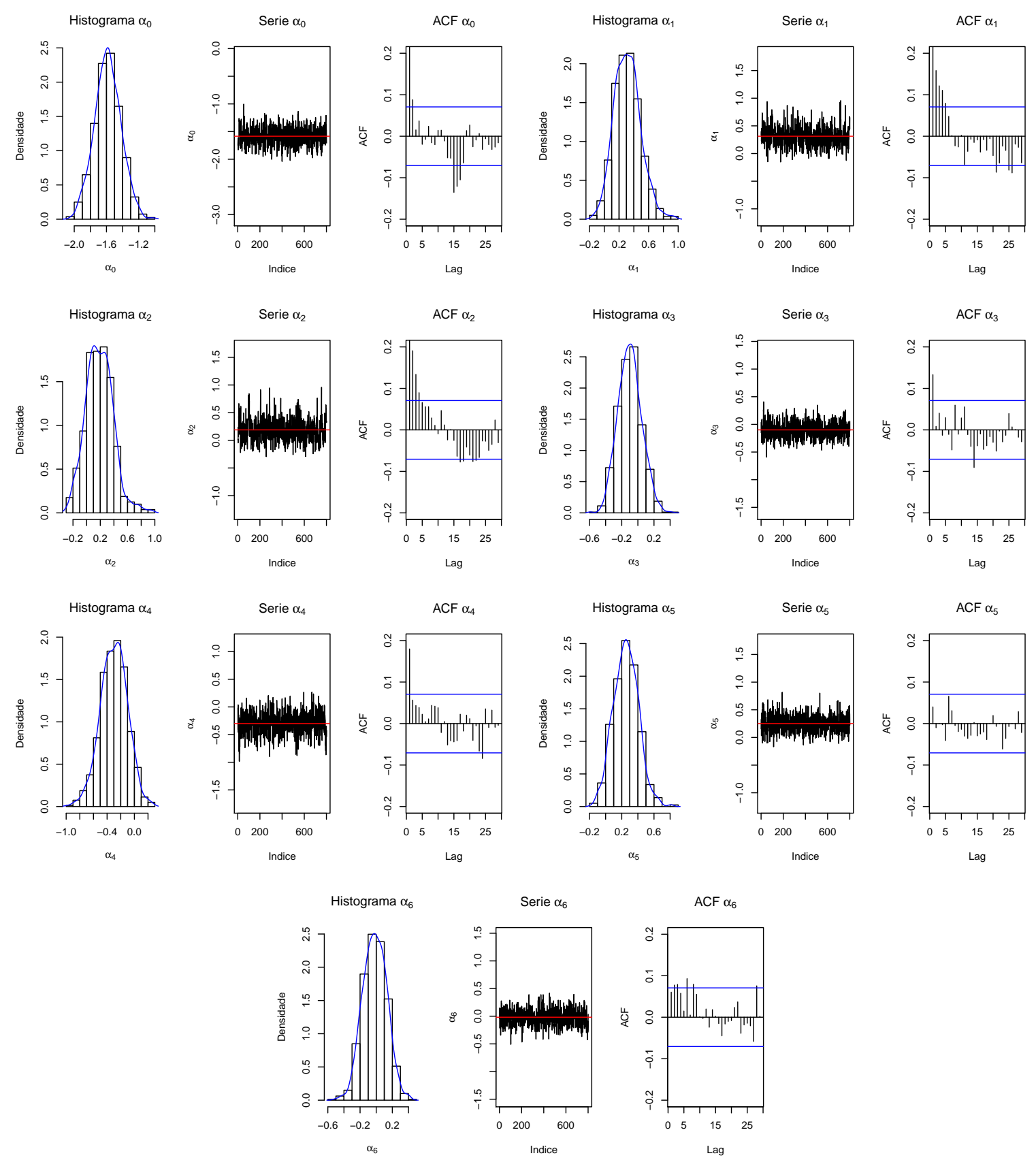

Figura D.4: Densidades a posteriori, traços e ACFs para a componente logito do ajuste do modelo fixo com $\left(J_{0}, J_{1}, J_{2}\right)=(15,15,15)$ segundo a cópula de Clayton aos dados de TMO. 


\section{D.2 Insuficiência renal crônica (IRC)}



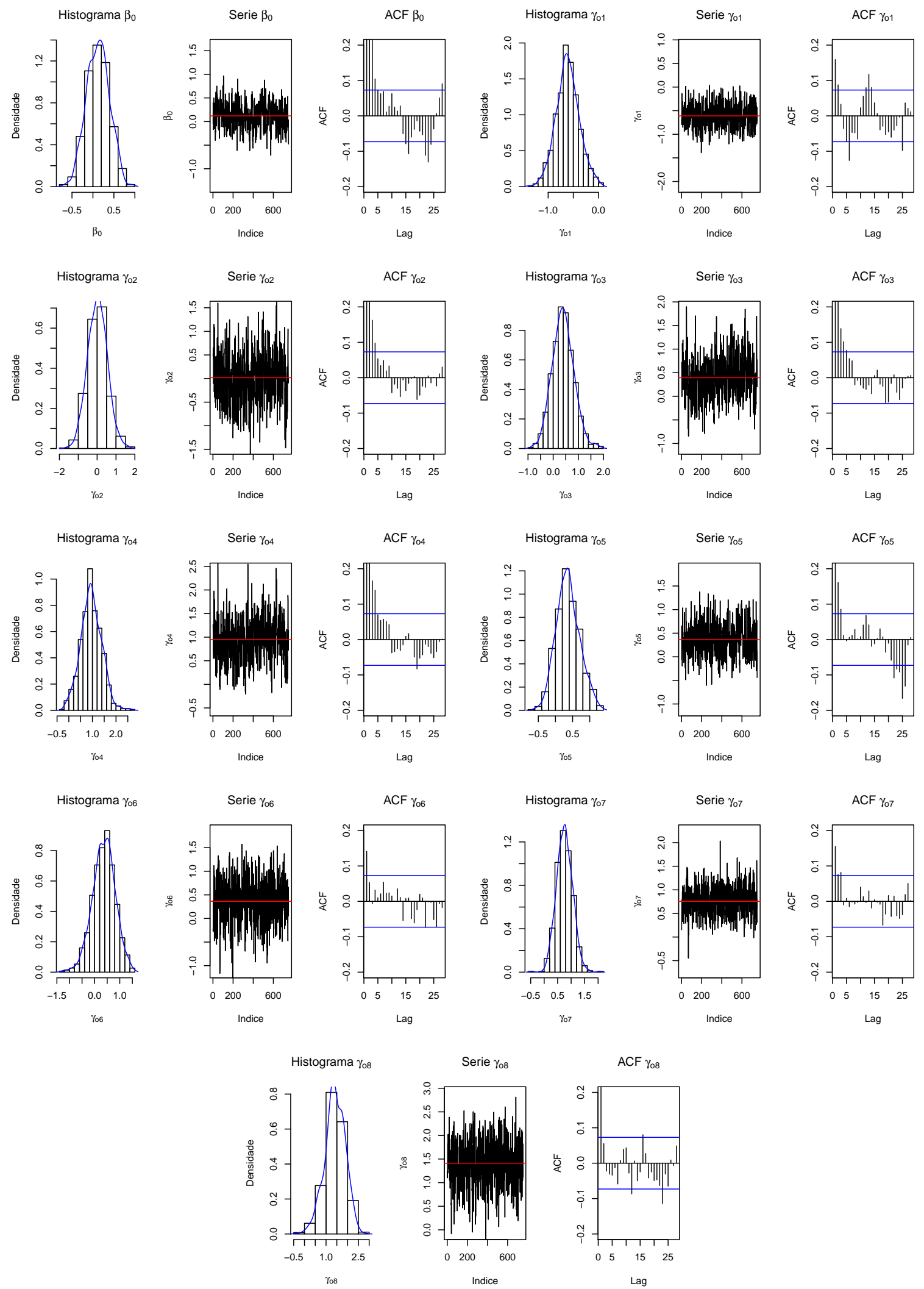

Figura D.5: Densidades a posteriori, traços e ACFs para a componente $T_{D}$ (Óbito) do ajuste do modelo fixo com $\left(J_{0}, J_{1}, J_{2}\right)=(2,2,2)$ segundo a cópula de Clayton aos dados de IRC. 

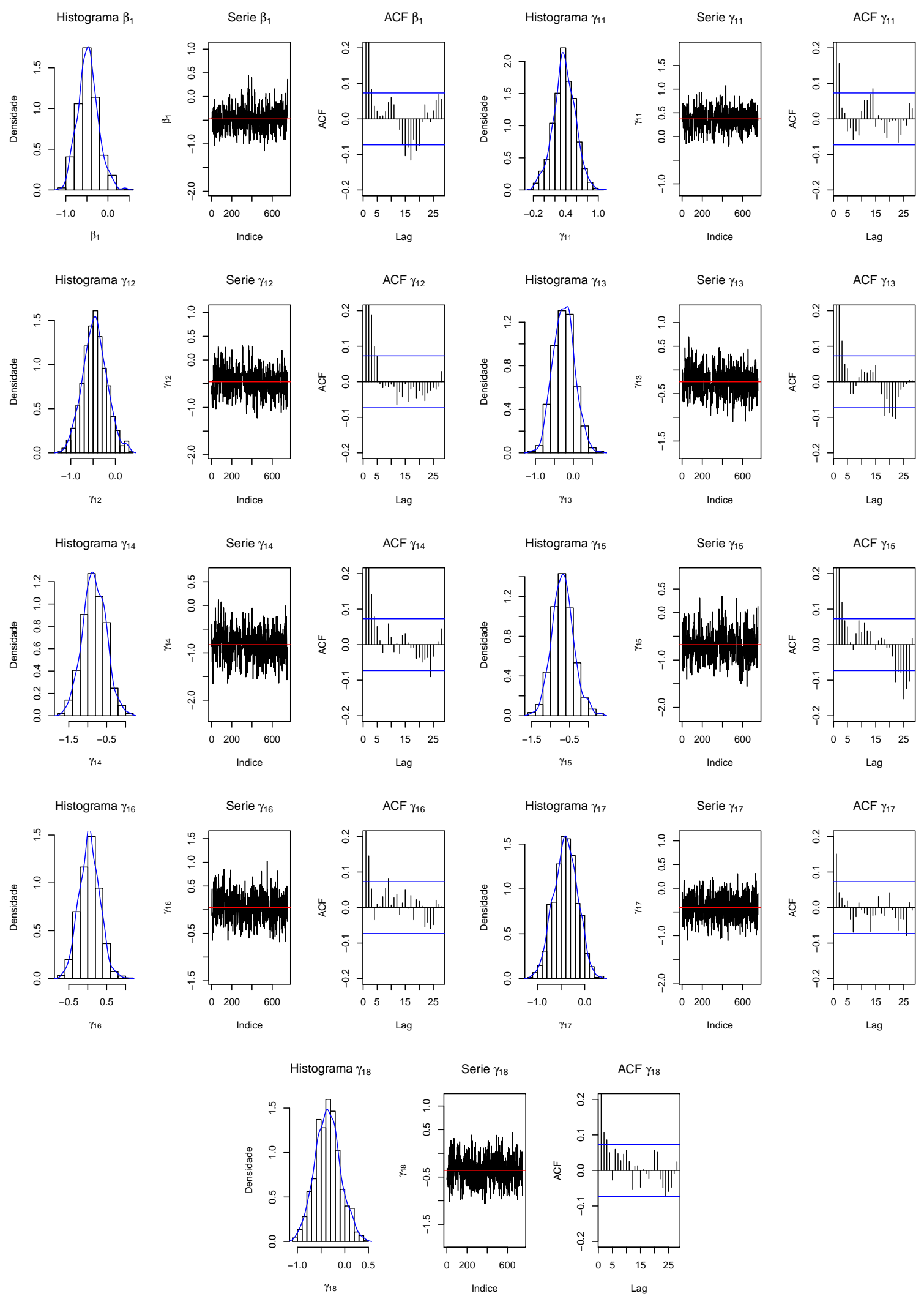

Figura D.6: Densidades a posteriori, traços e ACFs para a componente $T_{E}$ (Progressão) do ajuste do modelo fixo com $\left(J_{0}, J_{1}, J_{2}\right)=(2,2,2)$ segundo a cópula de Clayton aos dados de IRC. 

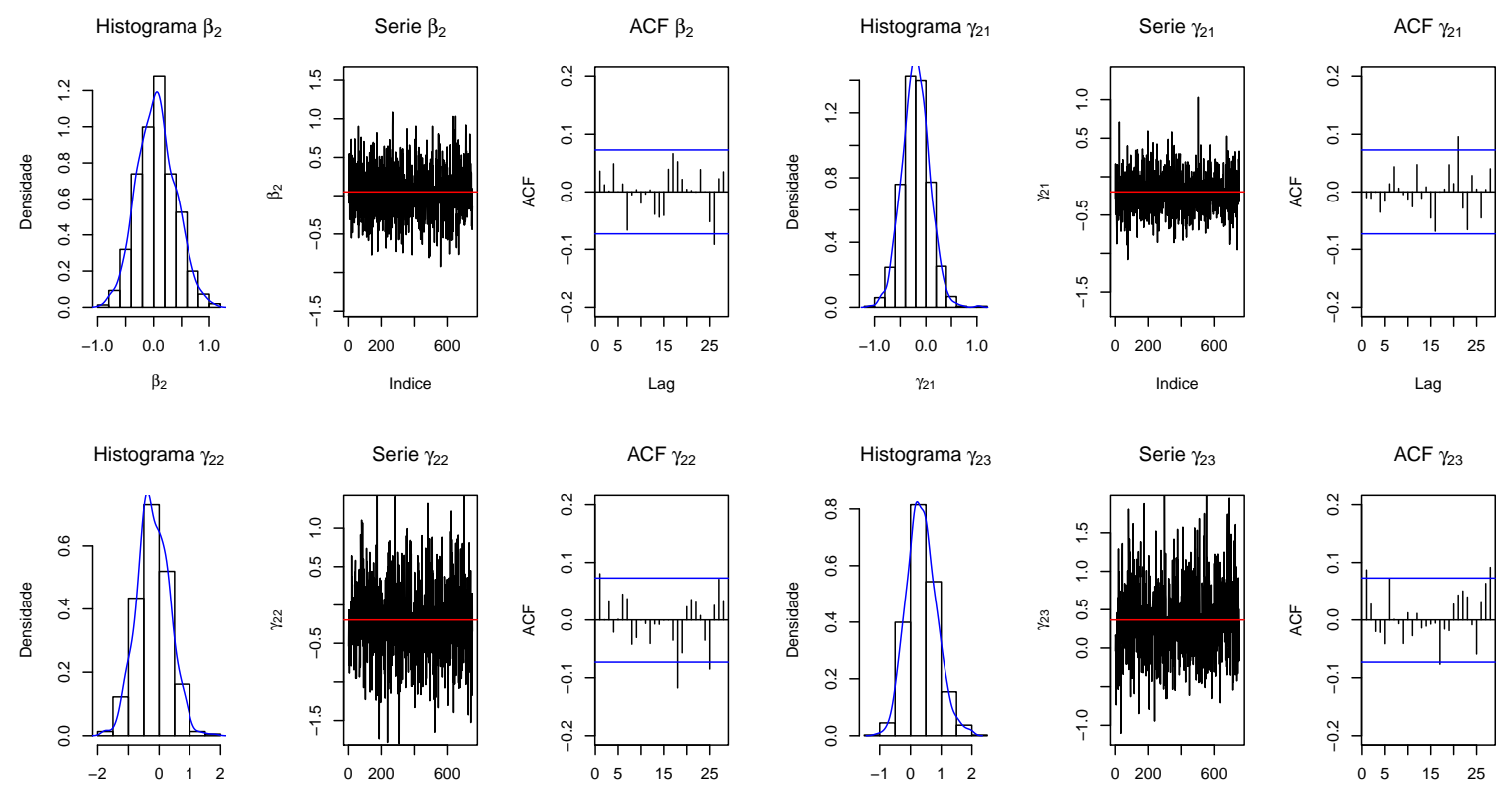

Histograma $\gamma_{24}$
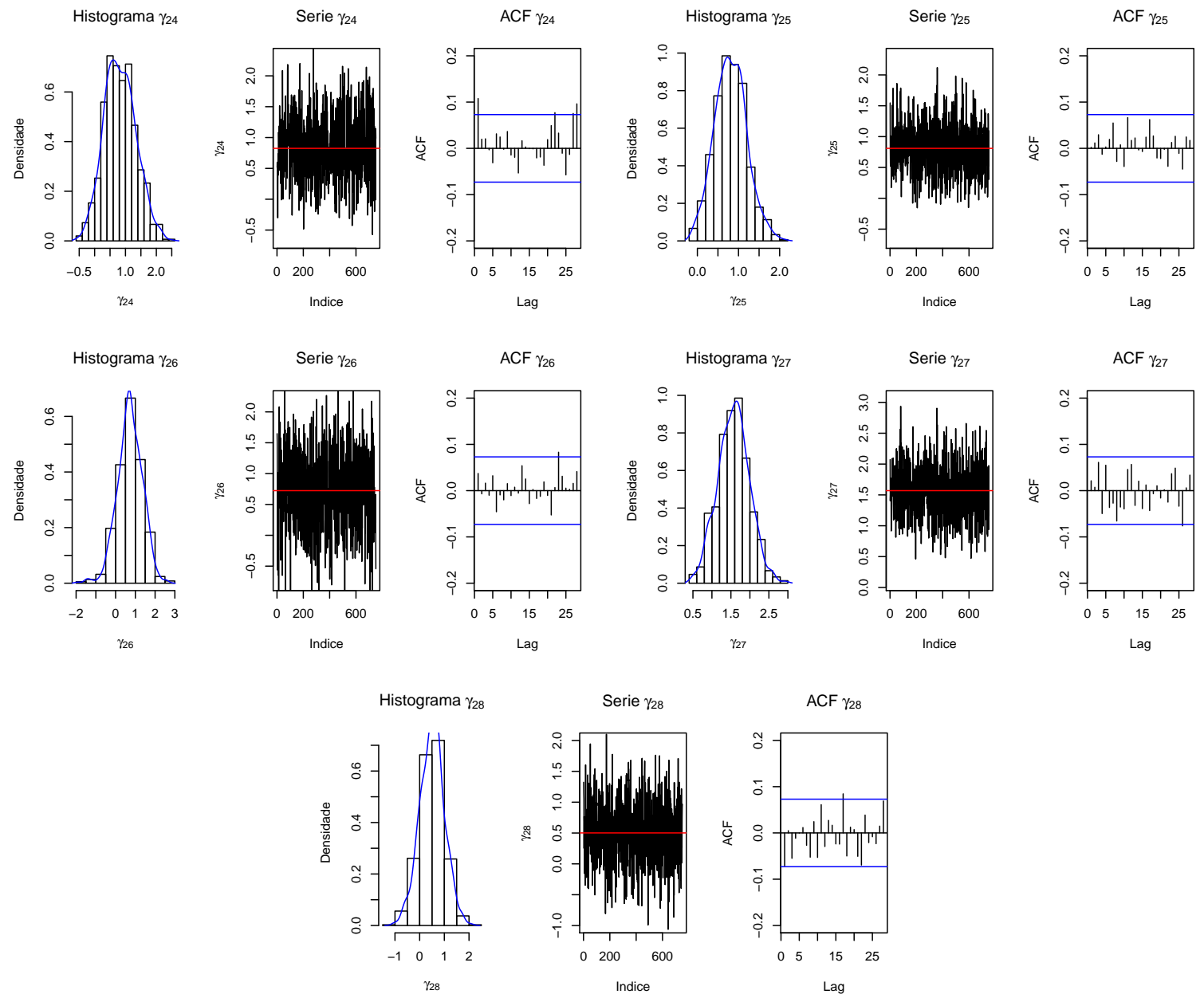

Figura D.7: Densidades a posteriori, traços e ACFs para a componente $T_{G}$ (Óbito após progressão) do ajuste do modelo fixo com $\left(J_{0}, J_{1}, J_{2}\right)=(2,2,2)$ segundo a cópula de Clayton aos dados de IRC. 

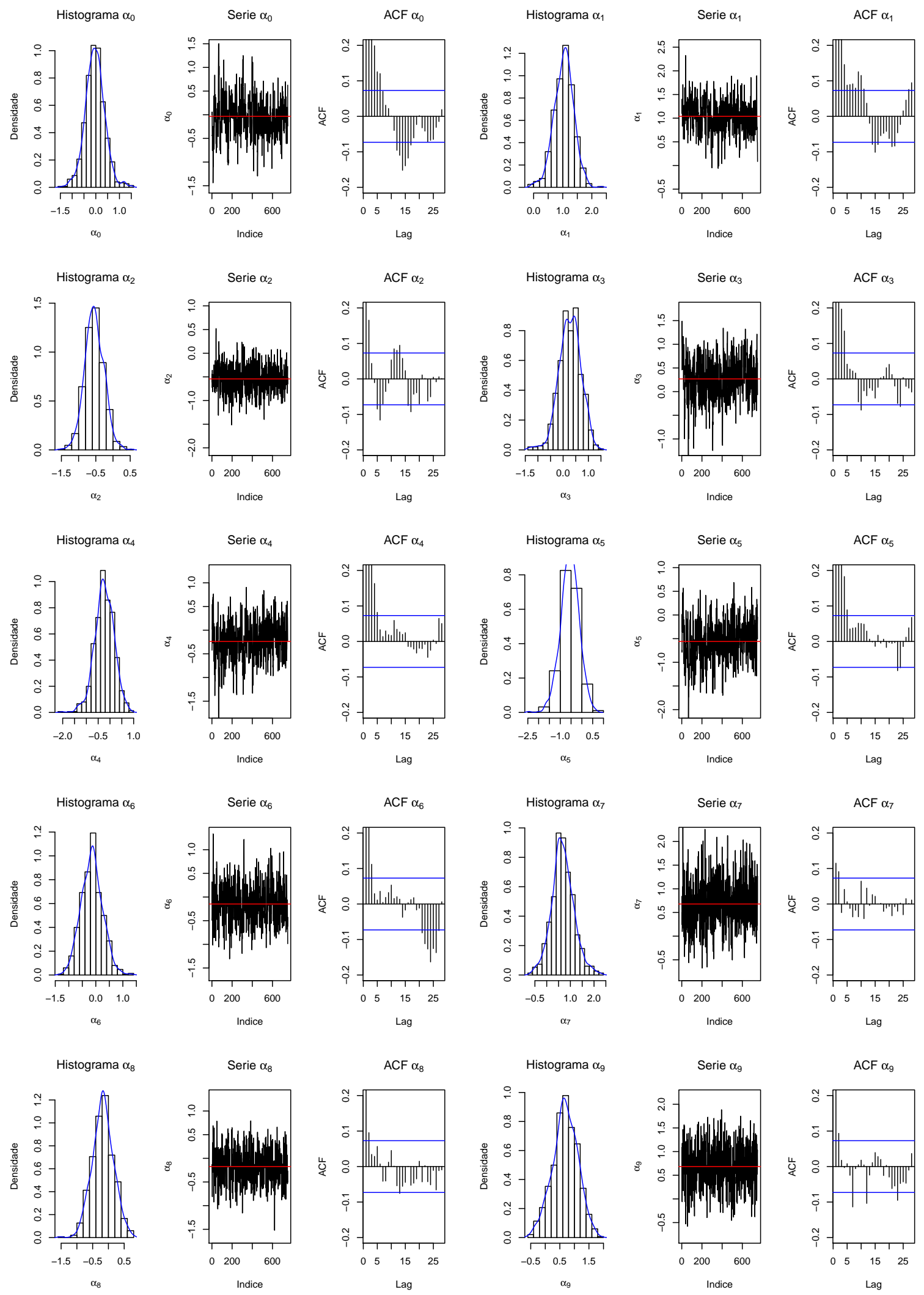

Figura D.8: Densidades a posteriori, traços e ACFs para a componente logito (Progressão) do ajuste do modelo fixo com $\left(J_{0}, J_{1}, J_{2}\right)=(2,2,2)$ segundo a cópula de Clayton aos dados de IRC. 

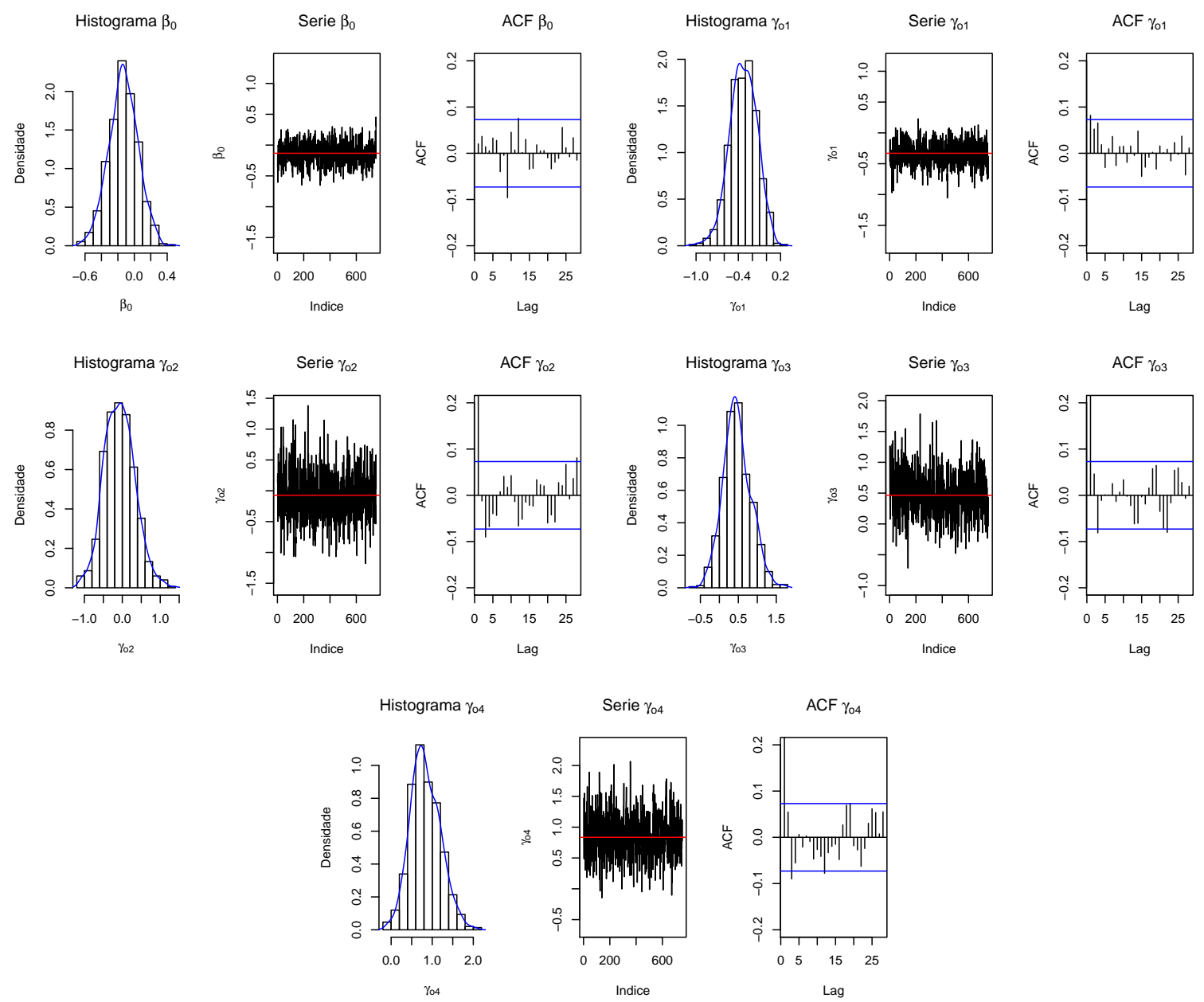

Figura D.9: Densidades a posterioris, traços e ACFs para a componente $T_{D}$ (Óbito) do ajuste do modelo misto com $\left(J_{0}, J_{1}, J_{2}\right)=(1,1,1)$ segundo a cópula de Clayton aos dados de IRC. 

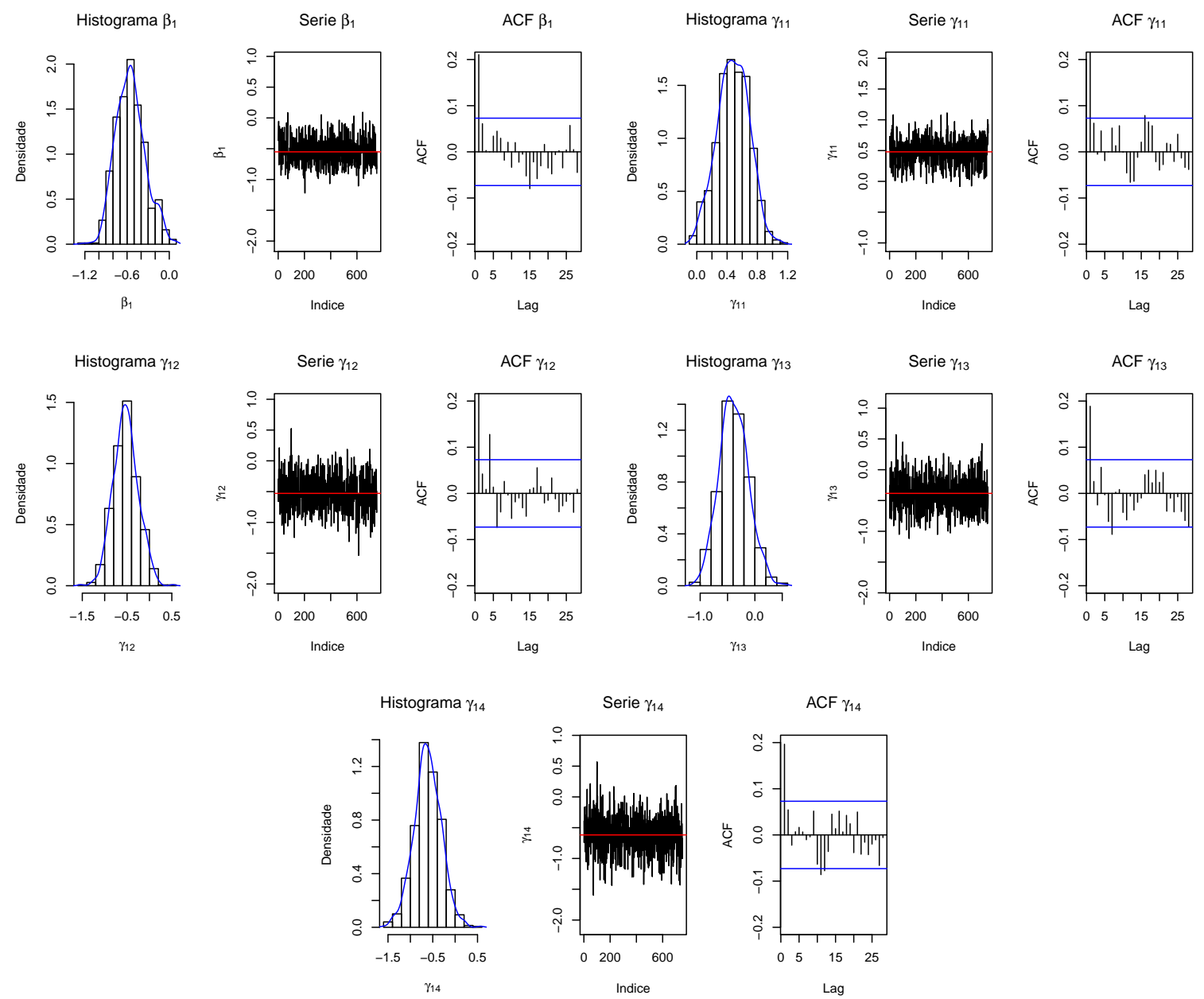

Figura D.10: Densidades a posteriori, traços e ACFs para a componente $T_{E}$ (Progressão) do ajuste do modelo misto com $\left(J_{0}, J_{1}, J_{2}\right)=(1,1,1)$ segundo a cópula de Clayton aos dados de IRC. 

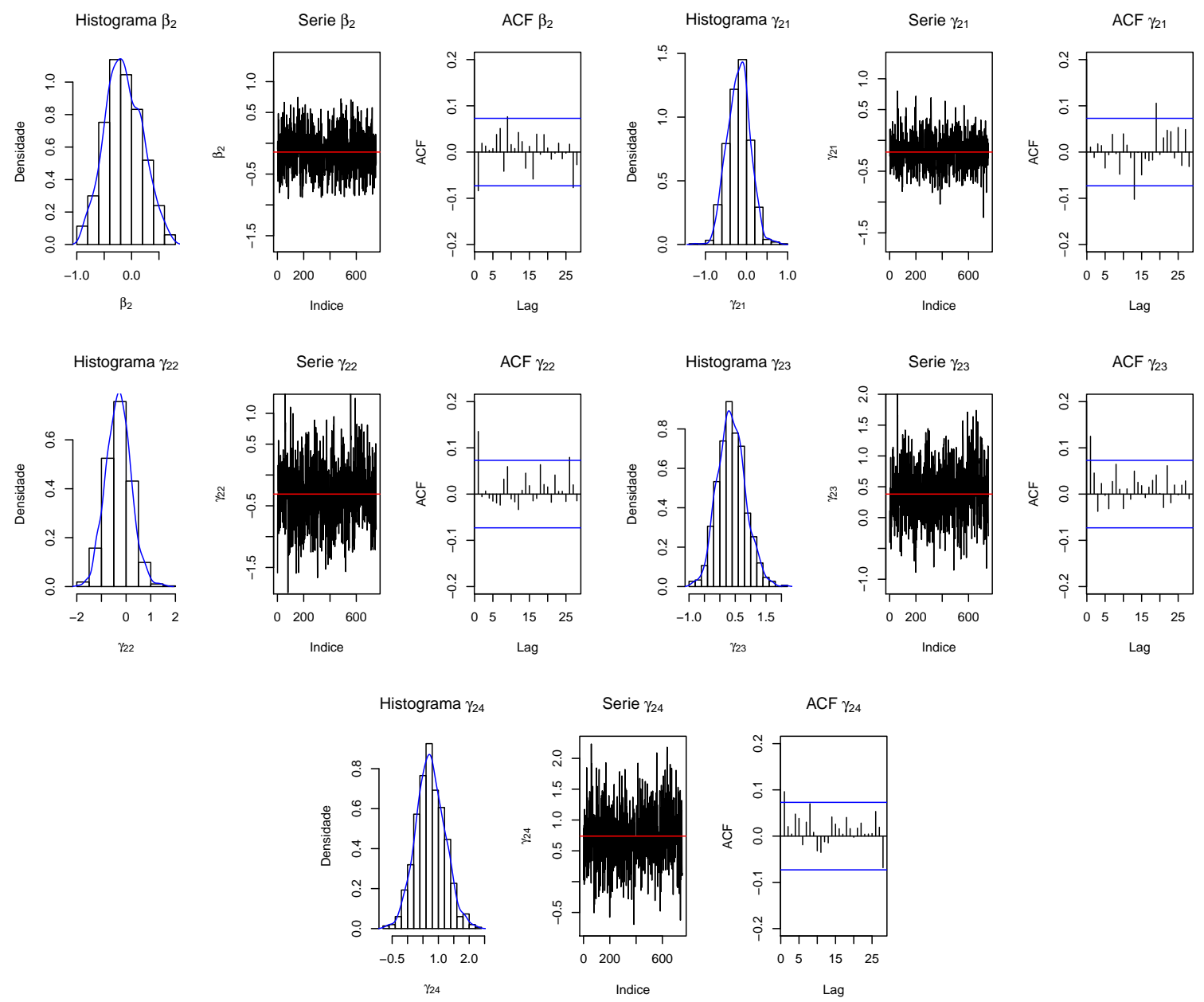

Figura D.11: Densidades a posteriori, traços e ACFs para a componente $T_{G}$ (Óbito após progressão) do ajuste do modelo misto com $\left(J_{0}, J_{1}, J_{2}\right)=(1,1,1)$ segundo a cópula de Clayton aos dados de IRC. 

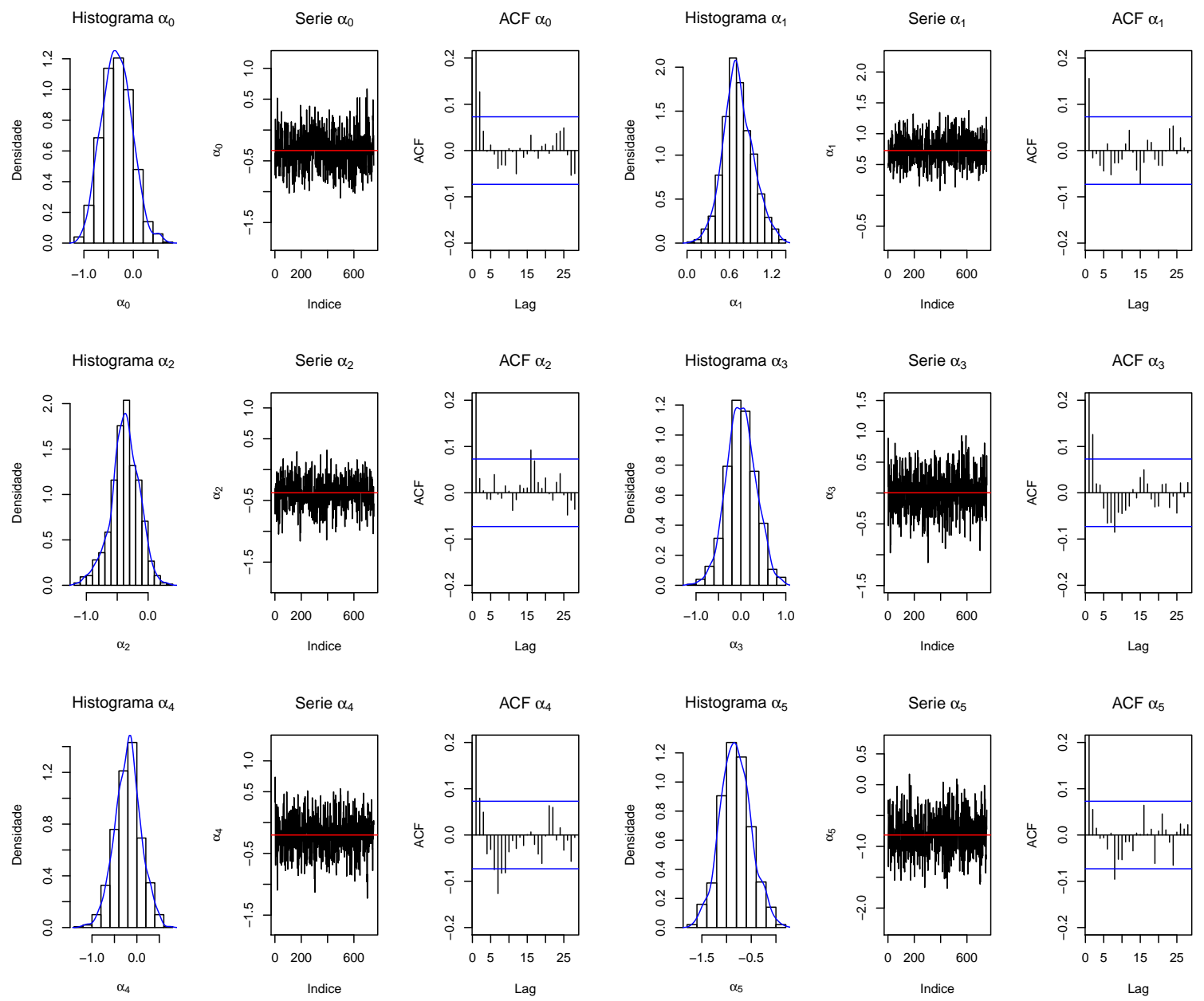

Figura D.12: Densidades a posteriori, traços e ACFs para a componente logito (Progressão) do ajuste do modelo misto com $\left(J_{0}, J_{1}, J_{2}\right)=(1,1,1)$ segundo a cópula de Clayton aos dados de IRC. 


\section{Referências Bibliográficas}

Bonat et al.(2012) W. H. Bonat, E. Teixeira, P. Justiniano e Marques W. Métodos computacionais para inferência com aplicações em $R$. Associação Brasileira de Estatística, ABE. Citado na pág. 29

Brechmann e Schepsmeier(2013) E.C. Brechmann e U. Schepsmeier. Modeling dependence with C- and D- Vine Copulas: The R Package CDVine. Journal of Statistical Software, 52:1-27. Citado na pág. 64

Burkardt(2014) J. Burkardt. The Truncated Normal Distribution.Department of Scientific Computing website. Florida State University. https://people.sc.fsu.edu/ jburkardt/presentations/truncated_normal.pdf, 2014. Citado na pág. 29

Casella e George(1992) G. Casella e E. I. George. Explaining the Gibbs sampler. The American Statistician, 46(3):167-174. Citado na pág. 5

Chen e Shao(2001) M-H. Chen e Q. Shao. Propriety of posterior distribution for dichotomous quantal response models. Em Proceedings of the American Mathematical Society, páginas 293-302. American Mathematical Society. Citado na pág. 104, 105

Chen et al.(2000) M-H. Chen, Q. Shao e J. Ibrahim. Monte Carlo Methods in Bayesian Computations. Springer-Verlag. New York. Citado na pág. 5, 72

Chen(2012) Y. Chen. Maximum likelihood analysis of semicompeting risks data with semiparametric regression models. Lifetime Data Analysis, 18:36-57. Citado na pág. 3

Colosimo e Ruiz(2006) E. A. Colosimo e S. Ruiz. Análise de Sobrevivência Aplicada. ABE Projeto Fisher. Edgard Blucher, Ltda. São Paulo. Citado na pág. 4, 19

Cox(1972) D. Cox. Regression models and life-tables. Journal of the Royal Statistical Society, Series B, 34(2):187-220. Citado na pág. 3

Cuenta del Alto Costo(2014) Cuenta del Alto Costo. Fondo Colombiano de Enfermedades de Alto Costo. Enfermedad Renal Crónica en Colombia. Resolución 4700 de 2008. Citygraf Impresores Ltda. Bogotá. Citado na pág. 4

de Castro et al.(2015) M. de Castro, M-H. Chen e Y. Zhang. Bayesian path specific frailty models for multi-state survival data with applications. Biometrics, 71:760-771. Citado na pág. 73 
de Wreede et al.(2011) LC. de Wreede, M. Fiocco e H. Putter. mstate: An R Package for the Analysis of Competing Risks and Multi-State Models. Journal of Statistical Software, 38 (7). Citado na pág. 73

Fine et al.(2001) J. P. Fine, H. Jiang e R. Chappell. On semi-competing risk data. Biometrika, 88:907-919. Citado na pág. 2, 5, 14, 15

Fiocco et al.(2008) M. Fiocco, H. Putter e HC. van Houwelingen. Reduced-rank proportional hazards regression and simulation-based prediction for multi-state models. Statistics in Medicine, 27:4340-4358. Citado na pág. 73

Frank(1979) M.J. Frank. On the simultaneous associativity of $f(x, y)$ and $x+y-$ $f(x, y)$. Aequationes Mathematicae, 19:194-226. Citado na pág. 11

Geisser(1993) A. E. Geisser. Predictive Inference: An Introduction. Chapman \&. Hall. New York. London. Citado na pág. 71

Geman e Geman(1984) S. Geman e D. Geman. Stochastic relaxation, Gibbs distributions, and the Bayesian restoration of images. IEEE Transactions on Pattern Analysis and Machine Intelligence, 6:721-741. Citado na pág. 29

Gilks e Wild(1992) W. R. Gilks e P. Wild. Adaptative rejection sampling for Gibbs sampling. Journal of the Royal Statistical Society. Series C (Applied Statistics), 41 (2):337-348. Citado na pág. 3, 29, 40

Gilks et al.(1995) W.R. Gilks, N. G. Best e K. K. C. Tan. Adaptative rejection Metropolis sampling within Gibbs sampling. Journal of the Royal Statistical Society. Series C (Applied Statistics), 44(4):455-472. Citado na pág. 40

Goethals et al.(2008) K. Goethals, P. Janssen e Duchateau L. Frailty models and copulas:similarities and differences. Journal of Applied Statistics, 35(9):1071-1079. Citado na pág. 5

Gómez(2006) Rafael Alberto Gómez. Renal disease in Colombia. Renal Failure, 28: 643-647. Citado na pág. 4

Hosmer et al.(2008) D. W. Hosmer, S. Lemeshow e S. May. Regression Modeling of Time-to-Event Data. John Wiley \&. Sons. New Jersey., 2nd ed. edição. Citado na pág. 19

Hsieh e Huang(2012) J. Hsieh e Y. Huang. Regression analysis based on conditional likelihood approach under semicompeting risks data. Lifetime Data Analysis, 18: 302-320. Citado na pág. 3

Hsieh et al.(2008) J. Hsieh, W. Wang e A. Ding. Regression analysis based on semicompeting risks data. Journal of the Royal Statistical Society. Series B, 70:3-20. Citado na pág. 3, 24

Ibrahim et al.(2000) J. G. Ibrahim, M-H. Chen e D. Sinha. Bayesian Survival Analysis. Springer-Verlag. New York. Citado na pág. 24, 72, 74 
Jiang et al.(2005) H. Jiang, J.P Fine, R. Kosorok e R. Chappell. Pseudo self-consistent estimation of a copula model with informative censoring. Scandinavian Journal of Statistics, 32:1-20. Citado na pág. 14, 15

Johnson et al.(1994) N.L. Johnson, S. Kotz e N. Balakrishnan. Continuous Univariate Distributions. John Wiley \&. Sons. New Jersey. Citado na pág. 29

Kalbfleisch e Prentice(2002) J. D. Kalbfleisch e R. L. Prentice. The Statistical Analysis of Failure Time Data. John Wiley \&. Sons. New Jersey., 2nd ed. edição. Citado na pág. 1, 4

Klein e Moeschberger(2003) J. P. Klein e M. L. Moeschberger. Survival Analysis: Techniques for Censored and Truncated Data. Springer-Verlag. New York., 2nd ed. edição. Citado na pág. 1, 4, 19

Lakhal et al.(2008) L. Lakhal, L. Rivest e B. Abdous. Estimating survival and association in a semicompeting risk model. Biometrics, 64:180-188. Citado na pág. 2, 3, 7, 8, $9,14,15,67,81$

Lawless(2002) J. F. Lawless. Statistical Models and Methods for Lifetime Data. John Wiley \&. Sons. New Jersey. Citado na pág. 19

Lee et al.(2015) K. H. Lee, S. Haneuse, D. Schrag e F. Dominici. Bayesian semiparametric analysis of semicompeting risks data: Investigating hospital readmission after a pancreatic cancer diagnosis. Journal of the Royal Statistical Society: Series C, 64: 253-273. Citado na pág. 1, 3, 7

Lee et al.(2016) K. H. Lee, F. Dominici e S. Schrag, D. ang Haneuse. Hierarchical models for semi-competing risks data with application to quality of end-of-life care for pancreatic cancer. Journal of the American Statistical Association, 111(515):10751095. Citado na pág. $1,3,72,73$

Lesaffre e Lawson(2012) E. Lesaffre e A. B. Lawson. Bayesian Biostatistics. John Wiley \&. Sons. New Delhi. Citado na pág. 28, 73

Lui(1994) J. S. Lui. The collapsed Gibbs sampler in bayesian computations with applications to a gene regulation problem. Journal of the American Statistical Association, 89(427):958-966. Citado na pág. 3

Martín-Cleary e Ortiz(2014) C Martín-Cleary e A Ortiz. Ckd hotspots around the world: where, why and what the lessons are. a ckj review series. Clinical Kidney Journal, 7:519-523. Citado na pág. 4

Neal(2003) R. M. Neal. Slice sampling. Annals of Statistics, 31:705-767. Citado na pág. 29,40

Nelsen(2000) R. Nelsen. An Introduction to Copulas. Springer-Verlag. New York., 2nd ed. edição. Citado na pág. 8, 9, 10 
Ntzoufras(2009) I. Ntzoufras. Bayesian modeling using WinBUGS. John Wiley \&. Sons. New Jersey. Citado na pág. 73

Oakes(1989) D. Oakes. Bivariate survival models induced by frailties. Journal of the American Statistical Association, 84:487-492. Citado na pág. 2

Paquet(2008) U. Paquet. Bayesian inference for latent variable models. Relatório Técnico UCAM-CL-TR-724, Computer Laboratory, University of Cambridge, Cambridge, United Kingdom. Citado na pág. 29

Patiño(2012) E. G. Patiño. Análise de dados com riscos semicompetitivos. Dissertação de Mestrado, Instituto de Matemática e Estatística, Universidade de São Paulo, Brasil. Citado na pág. 1, 4, 7, 15, 19, 81, 91

Peng e Fine(2007) L. Peng e J. Fine. Regression modeling of semicompeting risks data. Biometrics, 63:96-108. Citado na pág. 3

R Core Team(2015) R Core Team. R: A Language and Environment for Statistical Computing. R Foundation for Statistical Computing. Vienna, Austria. Citado na pág. 59

Rivest e Wells(2001) L-P. Rivest e M. T. Wells. A martingale approach to the copulagraphic estimator for the survival function under dependent censoring. Journal of Multivariate Analysis, 79:138-155. Citado na pág. 14, 15

Robert e Casella(2004) C. P. Robert e G. Casella. Monte Carlo Statistical Methods. Springer-Verlag. New York., 2nd ed. edição. Citado na pág. 33

Romeo(2005) J. S. Romeo. Modelagem Bayesiana para dados de sobrevivência bivariados através de cópulas. Tese de Doutorado, Instituto de Matemática e Estatística, Universidade de São Paulo, Brasil. Citado na pág. 2, 8, 11

Romeo et al.(2006) J. S. Romeo, N. I. Tanaka e Pedroso de Lima A. C. Bivariate survival modeling: a Bayesian approach based on Copulas. Lifetime Data Analysis, 12:205-222. Citado na pág. 8, 43

Romeo et al.(2018) J. S. Romeo, R Meyer e D. I. Gallardo. Bayesian bivariate survival analysis using the power variance function copula. Lifetime Data Analysis, 24:355383. Citado na pág. 93

Sahu e Dey(2000) S. K. Sahu e D. K. Dey. A comparation of frailty and other models for bivariate survival data. Lifetime Data Analysis, 6:207-228. Citado na pág. 5, 72

Schepsmeier et al.(2015) U. Schepsmeier, E. C. Brechmann, T. Erhardt e R Core Team. Cdvine: Statistical inference of c- and d-vine copulas. https://cran.rstudio. com/web/packages/CDVine/index.html, 2015. R package version 1.4. Citado na pág. 64

Schwarz(1978) G. Schwarz. Estimating the dimension of a model. Annals of Statistics, 6:461-464. Citado na pág. 5 
Sklar(1959) A. Sklar. Fonctions de répartition à $n$ dimensions et leurs marges. $P u$ blications de l'Institut de statistique de l'Université de Paris, 8:229-231. Citado na pág. 8

Spiegelhalter et al.(2002) D. J. Spiegelhalter, N. G. Best, B. P. Carlin e A. van der Linde. Bayesian measures of model complexity and fit. Journal of the Royal Statistical Society, Series B, 64:583-639. Citado na pág. 5, 73

Steddon e Sharples(2015) S. Steddon e E. Sharples. Clinical practice guideline. ckd - mineral and bone disordes - (ckd-mbd). http://www.renal.org/guidelines/ current-guidelines, 2015. [em linha] p. 11. Citado na pág. 78

Tanner e Wong(1987) T.A. Tanner e W. H. Wong. The calculation of posterior distributions by data augmentation. Journal of the American Statistical Association, 82:528-549. Citado na pág. 29

Varadhan et al.(2014) R. Varadhan, Q. Xue e K. Bandeen-Roche. Semicompeting risks in aging research: methods, issues and needs. Lifetime Data Analysis, 20(4): 538-562. Citado na pág. 7

Wang(2003) W. Wang. Nonparametric estimation of the sojourn time distributions for a multipath model. Journal of the Royal Statistical Society: Series B, (65):921-935. Citado na pág. 7

$\mathrm{Xu}$ et al.(2010) J. Xu, J. Kalbfleisch e B. Tai. Statistical analysis of illness-death processes and semicompeting risks data. Biometrics, 66:716-725. Citado na pág. 2, 3, 7, 8,74

Yan(2007) J. Yan. Enjoy the joy of copulas: with a package copula. Journal of Statistical Software, 21(4):1-21. Citado na pág. 11, 12

Zeng et al.(2012) D. Zeng, Q. Chen, M-H. Chen, J. Ibrahim e Amgen Research Group. Estimating treatment effects with treatment switching via semicompeting risks models: an application to a colorectal cancer study. Biometrika, 99:167-184. Citado na pág. $1,3,19$

Zeng e Karim(1991) S. L. Zeng e M. R. Karim. Generalized linear models with random effects; a Gibbs sampling approach. Journal of the American Statistical Association, 86:79-86. Citado na pág. 4, 28, 30

Zhang et al.(2014) Y. Zhang, M-H. Chen, J. G. Ibrahim, D. Zeng, Q. Chen, Z. Pan e $\mathrm{X}$. Xue. Bayesian gamma frailty models for survival data with semicompeting risks and treatment switching. Lifetime Data Analysis, 20:76-105. Citado na pág. 1, 3, 5, 19, $20,24,28,63,67,74,91$

Zheng e Klein(1982) M. Zheng e J. Klein. Estimates of marginal survival for dependent competing risks based on an assumed copula. Biometrika, 82:127-138. Citado na pág. 14,15 